\title{
Stress resilience : learning from imaging the brain
}

Citation for published version (APA):

Quaedflieg, C. W. E. M. (2016). Stress resilience : learning from imaging the brain. [Doctoral Thesis, Maastricht University]. Maastricht University. https://doi.org/10.26481/dis.20160121cq

Document status and date:

Published: 01/01/2016

DOI:

10.26481/dis.20160121cq

Document Version:

Publisher's PDF, also known as Version of record

\section{Please check the document version of this publication:}

- A submitted manuscript is the version of the article upon submission and before peer-review. There can be important differences between the submitted version and the official published version of record.

People interested in the research are advised to contact the author for the final version of the publication, or visit the DOI to the publisher's website.

- The final author version and the galley proof are versions of the publication after peer review.

- The final published version features the final layout of the paper including the volume, issue and page numbers.

Link to publication

\footnotetext{
General rights rights.

- You may freely distribute the URL identifying the publication in the public portal. please follow below link for the End User Agreement:

www.umlib.nl/taverne-license

Take down policy

If you believe that this document breaches copyright please contact us at:

repository@maastrichtuniversity.nl

providing details and we will investigate your claim.
}

Copyright and moral rights for the publications made accessible in the public portal are retained by the authors and/or other copyright owners and it is a condition of accessing publications that users recognise and abide by the legal requirements associated with these

- Users may download and print one copy of any publication from the public portal for the purpose of private study or research.

- You may not further distribute the material or use it for any profit-making activity or commercial gain

If the publication is distributed under the terms of Article $25 \mathrm{fa}$ of the Dutch Copyright Act, indicated by the "Taverne" license above, 


\section{STRESS RESILIENCE LEARNING FROM IMAGING THE BRAIN CONNY QUAEDFLIEC}

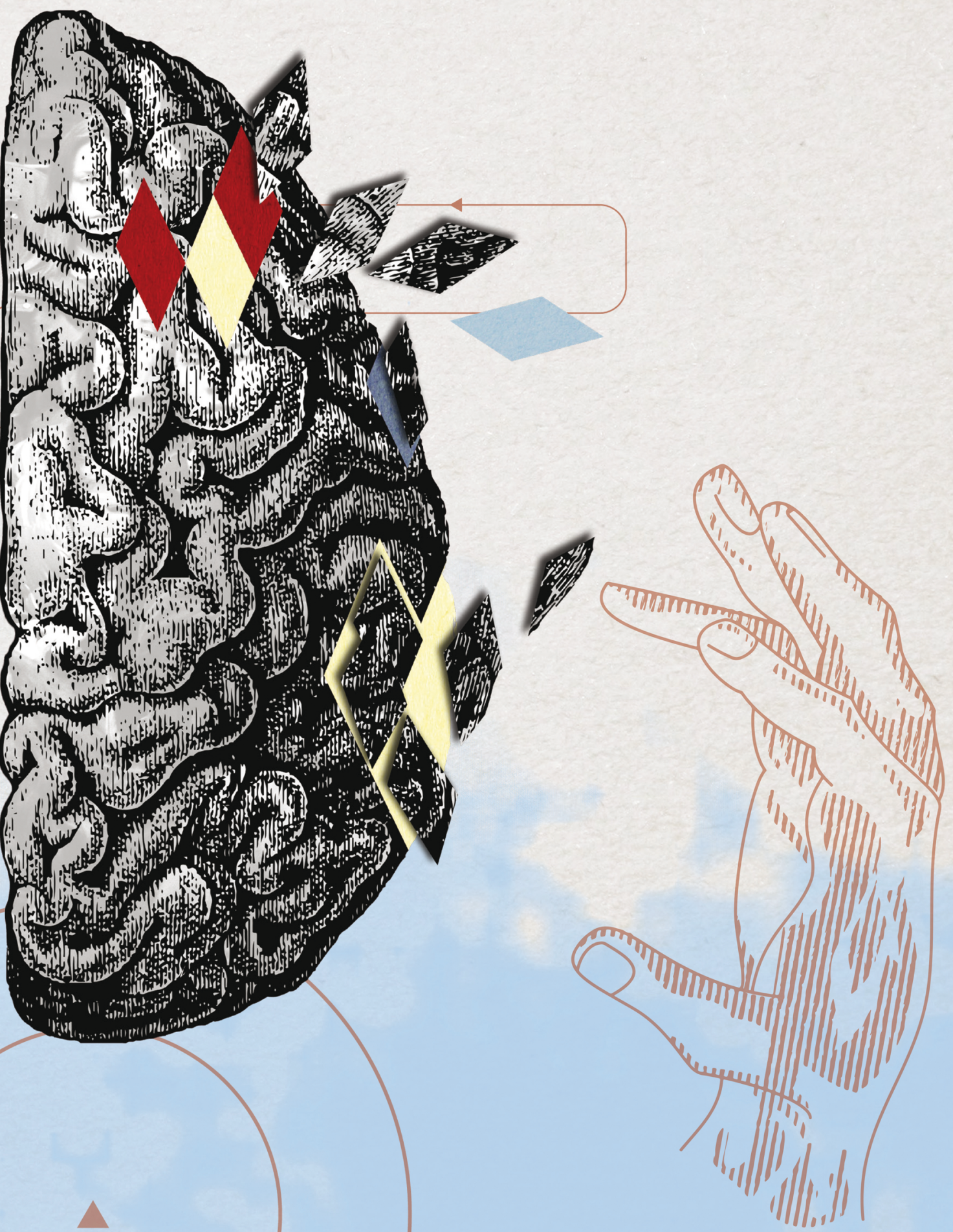




\section{Stress Resilience}

\section{Learning from imaging the brain}

1. The iMAST is an excellent tool to systematically investigate the timedependent mediating role of cortisol in the brain's response to a stressor (Chapter 1).

2. Resting state functional connectivity ( $\mathrm{rsFC})$ is informative in characterizing the temporal trajectory of cortisol-induced changes in brain connectivity during stress adaptation.

3. Timing is important. This is certainly true in the realm of how stress affects memory formation (Chapter 3).

4. The moderating role of lateralised frontal activity on acute stress responses is state-dependent, with relatively more left-sided activation characterising resilience (Chapter $2 \& 4$ ).

5. Deriving frontal EEG asymmetry from the individual alpha band is key in accounting for lateralised individual differences in the processing of stressors by the brain (Chapter 4).

6. Ensuring reproducibility on the individual level is a fundamental challenge in neurofeedback studies (Chapter 5).

7. Placebo controlled studies and comparisons with other established therapies are necessary before any translation of neurofeedback to a more applied context is justified.

8. With modern society becoming increasingly more demanding and complex, knowledge about successful adaptation to stress also becomes progressively more important.

9. Running a neurofeedback study is an excellent intervention method to train your own stress resilience.

10. Karakter wint van talent, als talent geen karakter heeft (Frank de Boer). 


\section{Stress Resilience}

Learning from imaging the brain

PROEFSCHRIFT

Ter verkrijging van de graad van doctor aan de Universiteit Maastricht, op gezag van de Rector Magnificus, Prof. Dr. L.L.G. Soete volgens het besluit van het College van Decanen, in het openbaar te verdedigen op

donderdag 21 januari 2016 om 14.00 uur

door

Cornelia Wilhelmina Elisabeth Maria Quaedflieg 


\section{CONTENTS}

Prof. Dr. H.L.G.J. Merckelbach

General Introduction

Part I

Dr. T. Smeets

\section{Beoordelingscommissie:}

Prof. Dr. M.L. Peters (voorzitter)

Prof. Dr. A.T. Sack

Prof. Dr. J.L. Kenemans

Prof. Dr. K. Roelofs

\section{Part II}

Chapter 4

Dr. E.H. Meijer

\section{Chapter 5}

General Discussion

Valorisation addendum

Summary

Samenvatting

Dankwoord

Curriculum vitae \&

Publications

Imaging the stressed brain

The imaging Maastricht Acute Stress Test (iMAST):

A neuroimaging compatible psychophysiological stressor

Temporal dynamics of stress-induced alternations

of intrinsic amygdala connectivity and

neuroendocrine levels

Time dependent effects of stress prior to encoding

on event-related potentials and $24 \mathrm{~h}$ delayed retrieval

\section{Brain asymmetry and stress resilience}

The functional role of individual-alpha based frontal asymmetry in stress responding

Validating frontal individual alpha asymmetry neurofeedback: Trainability, specificity, stability and interpretability 


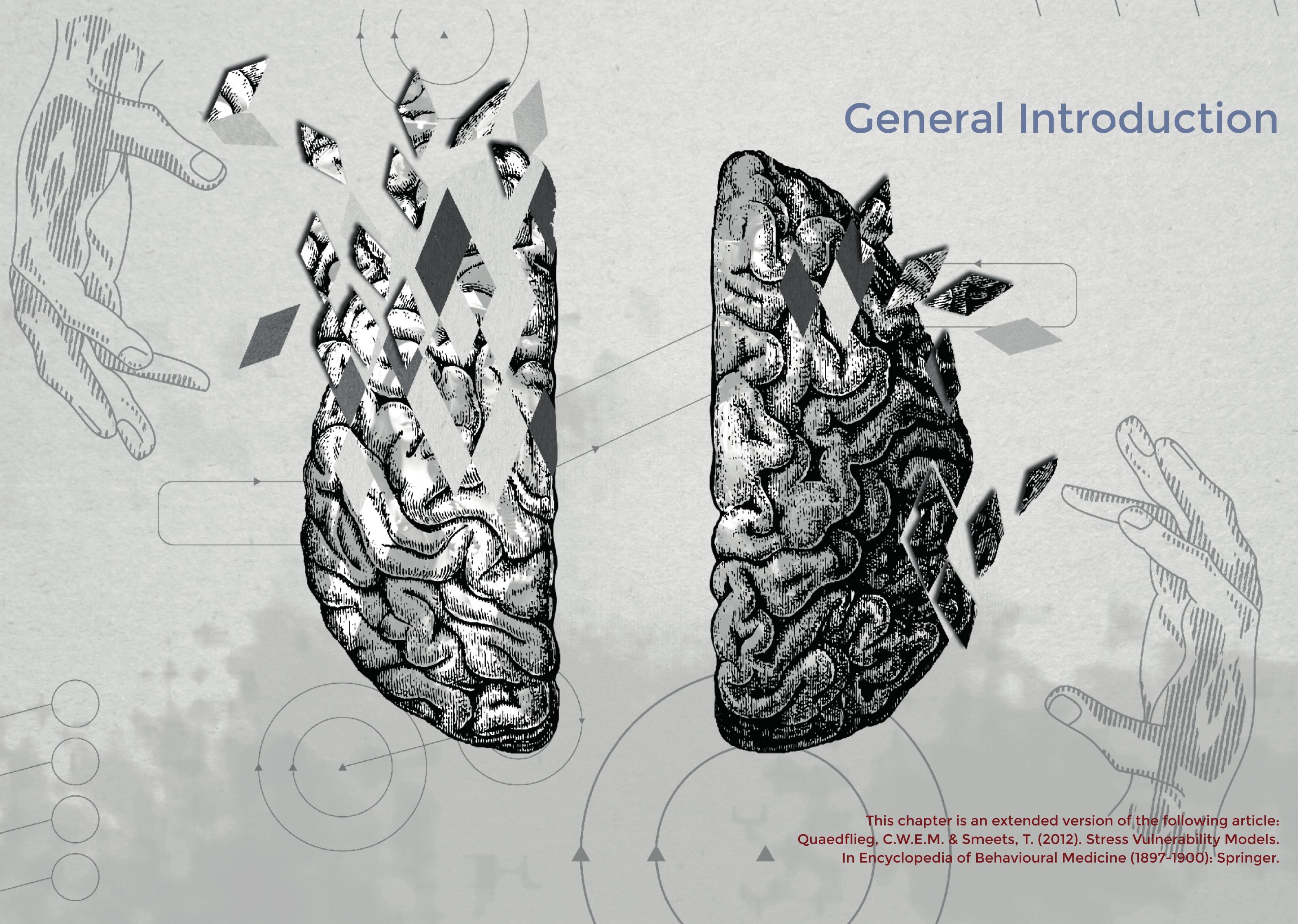


We all know how it feels to be stressed. Our lives are filled with daily hassles such as rushing for an appointment for which you are already late, deadlines at work or taking an exam at school, giving a presentation in front of a large audience, or being stuck in a traffic jam. Even planning a holiday or going to the dentist can be daunting to some of us. Thus, a wide range of events can provoke stress, and the amount of stress that is experienced in any given situation can differ between individuals. The ability to adapt well to stressful events has been coined resilience ${ }^{1,2}$. Resilience can be seen as a continuum that involves a dynamic process and depends on how we interpret the situation. After decades of investigating factors that put people at risk, researchers gained interest in studying factors that protect people from the development of mental Illness after a stressful experience. Stress vulnerability models assert that biological, social, and psychological factors will determine resilience ${ }^{3}$. In particular, these models emphasize that the impact of stressful life events depends on the nature and intensity of the stressor on the one hand, and on genetic and phenotypic risk and protective factors on the other hand. Psychosocial factors during development like social support, parental care and affective regulation style have been identified as potentially phenotypic protective factors that can enhance adaptive coping during or after stress $^{4-11}$.

Stress is said to be experienced when we are confronted with a physical or psychological threat. The immediate reaction to a physical or psychological threat consists of an emotional response, which is expressed as subjective withdrawal motivation, and a cognitive response, which involves attentional focusing on threat-related stimuli. After this immediate period in which survival is promoted, regulatory actions directed at restoring homeostasis are initiated. In particular, two physiological brain systems act in concert to restore homeostasis and jointly enable the individual to adjust the response to the stressor. The rapidly acting autonomic nervous system (ANS) results in the release of catecholamines (e.g., (nor)adrenaline) stimulating arousal, alertness, and focused attention. In turn, the activation of the slower hypothalamic-pituitary-adrenal (HPA) axis initiates a cascade of neurochemical events that results in the secretion of the glucocorticoid stress hormone cortisol from the adrenal glands (see Figure 1) 12,13. On the whole, the stress system is an integrated system, initially releasing catecholamines preparing you for the "fight-or-flight" response followed by the cortisol response that comes on-line during the selection of a proper behavioural response to a stressor. 


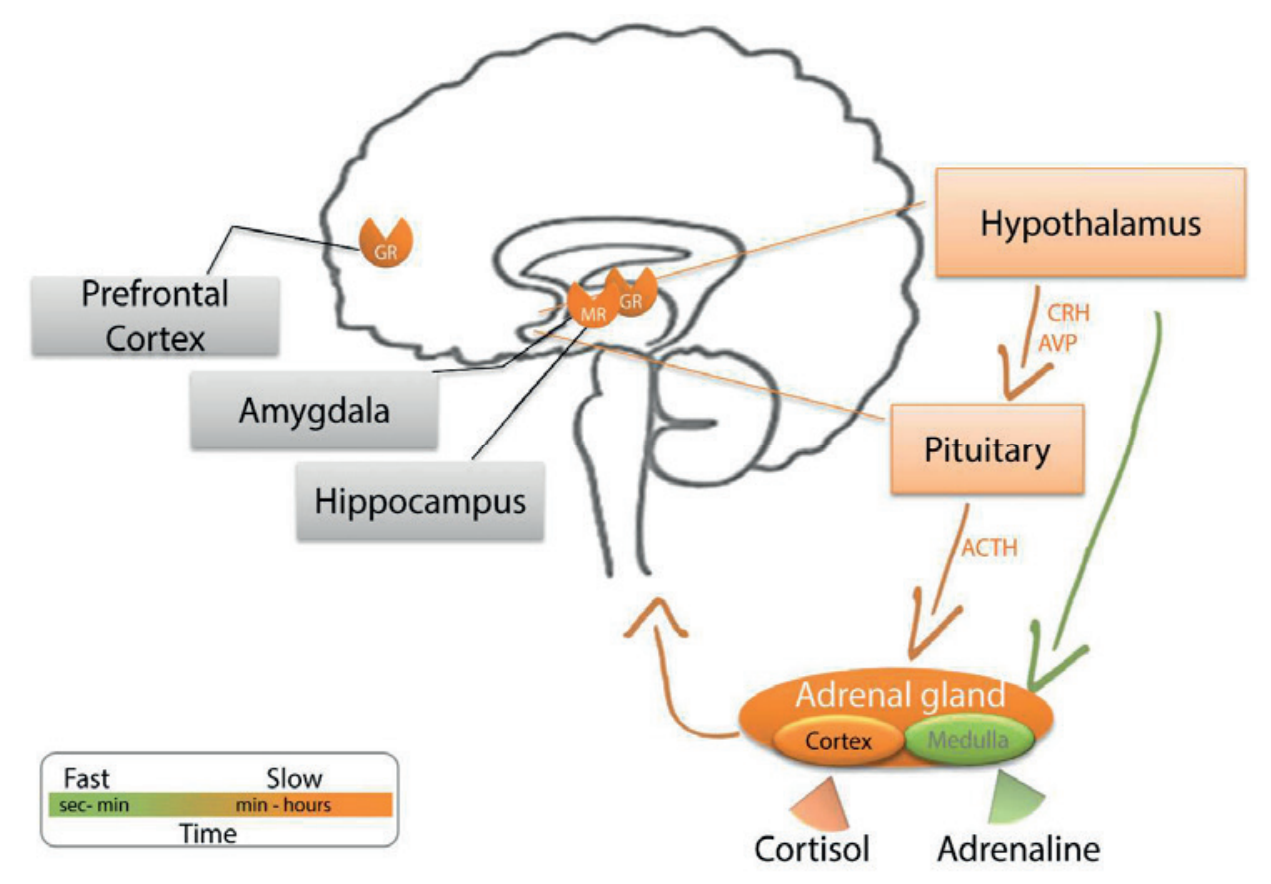

Figure 1. Overview of the stress system and the brain structures that play a key role in the stress response.

Within seconds following stress exposure, the sympathetic branch of the autonomic nervous system (ANS) is activated. Originating in the hypothalamus, activation of the sympathoadreno-medullary (SAM) axis results in the release of adrenaline, resulting in a bodily reaction (e.g., increased heart rate, respiration, and blood pressure) and enhanced alertness, arousal and attention. This ANS driven enhanced arousal state normalizes soon after stressor offset (i.e., it lasts only several minutes; denoted in green in the figure). The second major stress system, the hypothalamic-pituitary-adrenal (HPA) axis, responds slower and the levels of its end product cortisol remain increased for a longer time (i.e., min to hours; denoted in orange in the figure). Corticotrophin-releasing hormone (CRH) that is secreted from the hypothalamus induces the release of adrenocorticotrophic hormone (ACTH) from the anterior pituitary. ACTH enters the bloodstream and stimulates the adrenal cortex to secrete cortisol. Subsequently, cortisol enters the brain where it binds to two types of receptors. The mineralocorticoid receptor (MR) and the glucocorticoid receptor (GR) differ in their affinity as well as in their distribution in the brain. The relative balance between the MR and GR receptors determines the threshold and termination of the HPA-axis response to stress. The MR controls the basal HPA-axis activity via negative feedback on the pituitary gland and hypothalamus. In contrast the GR promotes recovery after stress as well as the storage of information for future events. Furthermore, GRs are widely expressed throughout the brain and especially in the prefrontal cortex and hippocampus, while the MRs are mainly localized in the limbic system. Consequently, stress effects on the brain are region-specific with the prefrontal cortex amygdala and hippocampus as main target areas of stress hormones.
Dysfunction of neuroendocrine regulation and impaired coping abilities have been implicated in a variety of psychiatric disorders and have been linked with a differential

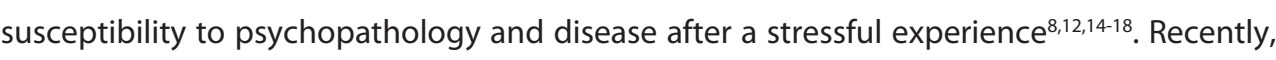
research started to use imaging methods as tools to investigate specific neural responses in the brain as protective or vulnerability factors in the development of psychopathology after stress. The aim of this thesis is to identify those changes in neural responding in the brain that underlie flexible adaptability after acute stress. At the heart of the described experiments are EEG and fMRI as brain imaging methods and the use of (i)MAST to induce acute stress in the laboratory. In part 1 of this thesis, "Imaging the stressed brain", we investigated the effects of acute stress on functional connectivity of the brain and on the neural correlates of memory formation. In part 2 of the thesis, "Brain asymmetry and stress resilience", we explored the possibility to modify cortical brain activity via self-regulation to provide an account for the role of our brain as possible protective factor in stress resilience.

\section{Stress vulnerability models}

To distinguish people who develop pathology from people who do not (i.e., are resilient), factors have been suggested that predispose individuals to psychopathology when confronted with severe stressors. In the late nineteen seventies, Zubin and Spring ${ }^{19}$ were the first to introduce this idea in the field of behavioural medicine by postulating a vulnerability model specific for schizophrenia. They suggested that humans inherit a predisposition to mental illness. However, an interaction between the genetic vulnerability and biological or psychosocial stressors is necessary to develop the disorder. The relationship between predispositional factors (or diathesis) and development of pathology has been described in four basic stress vulnerability models ${ }^{3}$.

The first and most simple stress vulnerability model, the dichotomous interactive model, contends that when predispositional factors are absent, even severe stress will not result in pathology. Instead, it is only when predispositional factors are present that stress may, depending on the severity of the stress, lead to the expression of pathology. Alternatively, the quasi-continuous model suggests varying degrees of predisposition with a continuous effect of predispositional factors on pathology once a threshold has been exceeded. The third, more extensive threshold model incorporates a specific individual threshold that is determined by the degree of vulnerability and the level of experienced 
stress. Finally, perhaps the most comprehensive model is the risk-resilience continuum model that incorporates different levels of severity of pathology by postulating a continuum that ranges from vulnerability to resilience. Here, resiliency characteristics that make people more resistant to the impact of stress are explicitly emphasized. Note that according to this latter model, even highly resilient individuals might still be at risk for developing pathology when experiencing extreme stress, but their individual threshold will be higher and the symptomatology of post-trauma pathology is likely to be less severe.

In general, stress vulnerability models postulate that a genetic vulnerability interacts with adverse life events or stressors to produce pathology. This gene-environment interaction with regard to stress and the development of pathology has been most extensively investigated in mood disorders such as depression. Gene-environment interaction studies use monozygotic twin, adoption, and family designs as tools to identify predispositional factors in shared and non-shared environments in order to differentiate genetic from environmental influences. In twin studies, a higher prevalence of pathology in monozygotic twins reared in different environments is used to confirm a genetic predisposition, whereas in adoption studies the effect of the environment (adoptive parents) can be offset against the effect of genes (biological parents). Using these methods, the heritability of major depression, for example, has been estimated at around $40 \%^{20}$. Moreover, genes can have a direct effect on the development of various brain systems. For instance, altered gene expression can reduce plasticity in brain circuits regulating mood, anxiety, and aggression, thereby decreasing one's ability to cope with stressful life events ${ }^{8}$. Genetic polymorphisms are then viewed as vulnerability factors given that they produce an increased sensitivity to the impact of stressful life events.

\section{The right tool for the job: Evoking stress in the neuroimaging environment}

Cortisol can be measured in our saliva and is used as a physiological stress marker in research to indicate that a laboratory stressor activated the stress system in the brain. Systematically investigating the mediating role of cortisol in the brain's response to a stressor requires a paradigm that effectively elicits robust cortisol responses. There are different kinds of stressors that are used to induce acute stress in a laboratory and that activate the two physiological stress systems to different degrees. For example, a physical stressor results in rapid activation of autonomic nervous system via reflexive mechanisms in brainstem and hypothalamus, while a psychological stressor requires processing in the prefrontal cortex (PFC) and in turn activates the HPA-axis via connections with limbic structures ${ }^{13,21}$. Extensive research has indicated that social evaluation, uncontrollability, and unpredictability are elements that make a task stressful ${ }^{22}$. Common laboratory paradigms used to reliably evoke stress in humans are the Trier Social Stress Test (TSST) ${ }^{23-25}$, the Cold Pressor Test (CPT) ${ }^{26,27}$, and the Socially Evaluated Cold Pressor Test (SECPT) ${ }^{28}$. The TSST consists of a short preparation period followed by a 5-min speech on any topic of participants' choice, and a 5-min mental arithmetic task, all performed in front of an audience while being audio- and videotaped. In the CPT, participants are instructed to immerse their hand up to the wrist in ice-cold (typically $0-5^{\circ} \mathrm{C}$ ) water for as long as possible with a maximum of 3-min. In the SECPT, participants perform the hand immersion task while being watched by an experimenter and being videotaped. The recently developed Maastricht Acute Stress Test (MAST) ${ }^{29}$ combines a physical (i.e., hand immersion in ice-cold water) and psychological (i.e. mental arithmetic challenges including psychosocial evaluative threat) stressor and has been shown to be a concise and valid procedure to reliably elicit robust subjective, autonomic and glucocorticoid stress responses.

Eliciting stress in a functional Magnetic Resonance Imaging (fMRI) environment, however, remains challenging ${ }^{30}$. Typical stress paradigms that have been used in brain imaging research include the serial subtraction task ${ }^{31,32}$ as well as emotional stimuli paradigms (e.g., emotional pictures ${ }^{33,34}$ or movies ${ }^{35-38}$ ), all of which result in rather modest cortisol increases compared to their non-imaging counterparts. Paramount among the imaging stress tests so far is the Montreal Imaging Stress Task (MIST) ${ }^{39,40}$, which is composed of a series of computerized mental arithmetic tasks with an induced failure algorithm and a social evaluative threat component. The (event)MIST thus incorporates key characteristics of psychological stress paradigms within the constraints of a neuroimaging environment and, compared to a control condition, elicits a significant cortisol stress response. Still, cortisol responses to the (event)MIST are considerably smaller than those of non-imaging stress protocols, and there is a large variation between studies applying the (event)MIST in the number of cortisol responders as well as in the maximal cortisol increase $\mathrm{e}^{39-45}$.

To elicit cortisol responses within the constraints of a neuroimaging environment more effectively than hitherto undertaken, we adapted the MAST to create a MRI compatible physically and psychologically challenging laboratory stress test. In chapter $\mathbf{1}$, we introduce 
this novel paradigm, which we labelled the imaging Maastricht Acute Stress Test (iMAST), and evaluate its effectiveness in evoking subjective and physiological stress responses. Basically, the iMAST consists of a 5-min preparation phase in which the task is explained and a 10-min acute stress phase that includes several exposures to cold-pressor stress and various mental arithmetic challenges along with social-evaluative pressure (i.e., negative feedback).

\section{The stressed brain}

Our brain perceives what is potentially threatening and hence, plays a key role in the capacity to cope with a stressor. Our brain regulates the acute stress response but vice versa acute stress also affects neuronal activity in the brain. Cortisol binds to two corticosteroid receptors in the brain, namely the mineralocorticoid receptor (MR) and the glucocorticoid receptor $(\mathrm{GR})^{12,13}$, and does so via two mechanisms. First, cortisol can bind to the hormone response element on DNA to influence gene expression (intracellular MR and GR binding properties). Second, cortisol can bind to membrane versions of the corticosteroid receptors to influence glutamate transmission and gene expression in the brain ${ }^{16}$. The MR controls the basal activity through inhibition of the HPA-axis ${ }^{46}$, facilitating the selection of adaptive behavioural responses and preventing minor adverse life events from disturbing homeostasis. In contrast, the GR promotes recovery after stress as well as the storage of information for future events. The relative balance between the MR and GR receptors determines the threshold and termination of the HPA-axis response to stress ${ }^{12,47}$. Studies have demonstrated that individuals with polymorphisms in the MR as well as in the GR gene display altered HPA-axis reactivity (i.e., increased or decreased cortisol secretion depending on the polymorphism) following standardized laboratory stress tests ${ }^{16,48,49}$, revealing a potential predisposition factor for stress-related pathology.

Human and animal studies have found wide-spread effects of stressors on brain regions that are also involved in emotion. These regions include, but are not limited to, the insula, hypothalamus, hippocampus, prefrontal cortex, cingulate cortex, and the amygdala ${ }^{50-53}$. As the fear centre of our brain, the amygdala is one of the fastest brain areas to react to a stressor. The amygdala is part of the salience network ${ }^{54}$ and excites the ANS and HPA responses, thus mediating the initial surge in vigilance and optimizing detection of threats for homeostasis ${ }^{12,37,55}$. The amygdala is also crucially involved in stress-induced longterm adaptive responses like enhanced memory consolidation ${ }^{56,57}$
Cortical brain regions modulate the response of the amygdala to physical or psychological threat. Physiological and imaging studies have demonstrated that the medial prefrontal cortex ( $\mathrm{mPFC}$ ) modulates the amygdala response during regulation of autonomic and affective responses ${ }^{58-65}$. Furthermore, several studies using different paradigms (i.e., trauma related sounds, photos, fearful faces, neutral memory tasks) have reported increased amygdala activation and decreased mPFC activation in post-traumatic stress disorder $(\mathrm{PTSD})^{66}$. Interestingly, resilience to PTSD in police officers was associated with increased mPFC and less amygdala activity during traumatic memory retrieval ${ }^{67}$. These findings, together with the idea that stress is a complex behavioural response mediated by networks of areas in the brain, suggests that the functional connectivity of the amygdala with mPFC might play a key role in stress resilience.

Functional connectivity of brain areas has been investigated using task absent (i.e., resting state) fMRI measurements (rs-fMRI) based on the rationale that regions exhibiting a high degree of coherence are functionally interconnected in terms of flow of neural information ${ }^{68}$. Resting state functional connectivity ( $r s-F C$ ) is especially useful to study the effect of post-stress brain activation patterns since the measured activity is not related to a task, enabling the exploration of diffuse effects of stress on the brain. Previous neuroimaging studies demonstrated that amygdala rs- $\mathrm{FC}$ is differentially modulated during acute stress $^{69}$ and the early recovery following a stressor ${ }^{70}$, with enhanced amygdala-mPFC coupling one hour after the stressor. In chapter $\mathbf{2}$ we confirm the potential of the iMAST to study the processing of stress signalling in the brain and investigate the moderating role of glucocorticoids on the change in amygdala rs-FC during two phases that follow stress exposure: the acute stress and early recovery phases.

\section{Stress and memory}

The end products of the rapid and delayed physiological stress systems, the ANS and HPAaxis, influence cognitive processes initiated by the brain, enabling the prioritization of adaptive cognitive processes after having been exposed to a stressor ${ }^{13}$. Enhanced memory consolidation is a well-known long-term adaptive behavioural consequence of stress exposure. The binding of cortisol to the corticosteroid receptors in the hippocampus but also the amygdala and other brain structures activates mechanisms involved in hippocampal plasticity, thereby influencing memory ${ }^{71,72}$. Moreover, stress has a more 
pronounced effect on emotional memory consolidation ${ }^{73-75}$ and this has been related to the interaction of cortisol with the noradrenergic system in the basolateral nucleus of the amygdala (BLA $)^{76-78}$. It is thought that connectivity between the amygdala and hippocampus is required for this enhanced emotional memory and that this connectivity generates and regulates emotional reactions to stressful stimuli ${ }^{79,80}$.

Cortisol plays a key role in the effects of stress on memory ${ }^{81,82}$ and exerts a time specific dual mode of action in the brain ${ }^{83,84}$. Importantly, these rapid non-genomic and delayed genomic glucocorticoid actions, have opposite effects on certain brain areas ${ }^{85}$. For instance, neuroimaging studies have demonstrated that rapid non-genomic cortisol effects enhance activity while delayed genomic effects suppress activity in the amygdala and hippocampus ${ }^{37,86}$. Acute stress sets the brain in a consolidation mode and thus enhances memory formation when it is experienced within the spatiotemporal context of the learning episode ${ }^{87}$. Indeed, Smeets and colleagues ${ }^{88,89}$ demonstrated that enhanced memory consolidation is dependent on the contextual relatedness between stressor and to-beremembered stimuli (i.e., enhanced recall of context-related words). Additionally, support for the temporal relatedness comes from a recent study by Zoladz and colleagues ${ }^{90}$, who examined the effects of stress applied immediately or 30 min before learning neutral, negative and positive words. They found that stress immediately before encoding enhanced recognition of positive words 24-h later, whereas stress 30-min before encoding impaired recall of negative words. One of the mechanisms behind this consolidation enhancement is a rapid increase of glutamate-mediated excitability in the hippocampus and the amygdala via noradrenergic and non-genomic membrane MR activity ${ }^{83,91}$. Following this period of potential memory enhancement, genomic GR actions induce a refractory state of the hippocampus, impairing the processing of new information ${ }^{71,87,92}$.

Thus, stress applied immediately before learning can be expected to enhance longterm memory by increased vigilance to (emotional) stimuli, while stress applied longer before learning would impair long-term memory. In chapter 3, we investigated the role of stress timing on emotional memory processing and its underlying neural basis by combining behavioural measures with electroencephalography (EEG) measurements. Event-related potentials (ERPs) obtained from EEG were used to assess cortical information processing and provide information about the time course of memory formation ${ }^{93,94}$.

\section{Brain asymmetry}

Lateralized processing in the prefrontal cortex is involved in the regulation of the neuroendocrine stress response $\mathrm{s}^{52,95,96}$. Specifically, based on animal and human neuroimaging studies, it is suggested that the right hemisphere initiates neuroendocrine and behavioural fight-or-flight responses, while the left hemisphere regulates them ${ }^{97-100 \text {, }}$ most probably through interhemispheric inhibition ${ }^{101}$. This is supported by patient and studies demonstrating increased cortisol levels in the morning after having had a stroke in the left prefrontal cortex ${ }^{102}$ and experimental studies showing increased phasic cortisol levels when presenting an emotional film to the right hemisphere ${ }^{103}$.

Lateralization of prefrontal cortex has also been linked to individual differences in processing of emotions and resilience. The left-frontal hemisphere has been associated with an approach system, activated when an individual is moving towards goals, and generates positive emotions with the purpose of reinforcing goal attaining behaviours ${ }^{104,105}$. Conversely, the right lateralised withdrawal system produces more negative affect intended to motivate the individual to move away from potentially dangerous situations or stimuli ${ }^{106,107}$. The approach and withdrawal systems could be related to differences in resilience, by how they bias motivational processing. For Example, Curtis and Cicchetti ${ }^{108}$ demonstrated that greater left frontal activity differentiated resilient from non-resilient (trauma exposed) children. Furthermore, frontal asymmetry measured during exposure to trauma reminders has been found to differentiate PTSD patients from resilient individuals ${ }^{109}$. Also, in healthy participants, we recently found that left-sided frontal activation predicted lower physiological responses to reminders of a trauma film, although this effect emerged only for one type of film (i.e., a severe car crash) and not for another (i.e., a genocide film) ${ }^{110}$.

A reliable correlate of frontal activity is frontal alpha oscillations measured through EEG recordings. Frontal alpha asymmetry refers to the average difference in $8-13 \mathrm{~Hz}$ activity between the left and right frontal areas across several minutes ${ }^{111}$. Frontal alpha asymmetry is most often measured while participants are at rest and reflects a dynamic recurring series of states ${ }^{112}$. It is suggested that alpha oscillations originate from thalamo-cortical circuits and play an important role in neuronal processing via inhibition, reducing the processing capabilities of a given area ${ }^{113-115}$. That is, the power of alpha oscillations is inversely related to brain activity. A brain that is said to be asymmetric to the right has an alpha predominance in the left hemisphere. 
The relation between lateralized brain activity and emotions or stressful life events has been supported by patient studies. These studies typically report higher right frontal activity in patients with depression ${ }^{116-119}$. Moreover, right frontal asymmetry predicted first depressive episode in healthy individuals in a three-year follow-up study ${ }^{120}$. Experimental studies also support the role of functional prefrontal cortex lateralisation in affect and stress regulation. For instance, greater left- than right frontal activity at baseline predicted greater emotional flexibility ${ }^{121}$, better emotion regulation ${ }^{122}$ and was associated with superior ANS response (i.e., increased cardiac output and decreased peripheral pressure) ${ }^{123}$. In the same vein, Lewis, Weekes, and Wang ${ }^{124}$ found that high examination stress increased relative right-sided frontal activity in the absence of changes in cortisol levels. Pharmacological studies have yielded mixed results, with cortisol administration resulting in either a shift to the right ${ }^{125}$ or to the left in a formal testing condition ${ }^{126}$. However, these studies provide indirect evidence for a role of frontal asymmetry in stress processing since a psychological stressor depends on processing in the brain and activates the HPA-axis at a different level than exogenous cortisol ${ }^{127}$.

In chapter 4, we investigated the functional role of asymmetric frontal alpha oscillations in task-induced stress responding. Additionally, we examined whether frontal asymmetry based on an individualized alpha frequency band can provide a more sensitive measure of individual differences in lateralized stress responding. It is known that there are inter-individual variations in the spectral frequencies ${ }^{128,129}$, specifically in alpha peak and bandwidth ${ }^{130-132}$. Moreover, individual differences in alpha peak frequency have been related to several cognitive functions including perception, attention, and memory ${ }^{133}$.

\section{Neurofeedback as a tool to target stress resilience}

Several psychopathological disorders display a characteristic brain activity profile, such as right-sided frontal asymmetry in depression (cf. supra). This altered brain activity profile has yielded a vested interest in treatment possibilities based on the brain neurophysiology through neurofeedback. Neurofeedback is based on the real-time analysis of ongoing brain activity and uses operant conditioning as the mechanism to shape the functional architecture of the working brain. Learning is achieved via auditory or visual feedback that reflects the current brain activity in the area of interest. The area of interest is based on knowledge about the neural mechanisms underlying a disorder or the desired behavioural change. The activity in the area of interest can be recorded with imaging methods, such as EEG and fMRI. The primary concern regarding the neurofeedback is to ensure that it represents the actual underlying neural activity rather than mere measurement artefacts. The auditory or visual feedback that is provided reinforces the desired brain activity, thereby teaching the patient to change their own brain activity and increasing the likelihood of the subsequent spontaneous re-occurrence of the desired brain activity ${ }^{134-139}$. There has been a recent surge in the use of EEG neurofeedback for treating psychopathological disorders. Nevertheless, EEG neurofeedback has been criticized as its alleged efficacy is often not evidence-based ${ }^{140}$. Most clinical neurofeedback studies are based on a small sample, are not double-blind, use invalid measures of symptoms, and do not include a control group or follow-up measures ${ }^{141,142}$. For instance, the evidence for the effectiveness of neurofeedback for attention-deficit/hyperactivity disorder (ADHD) is found to be less robust when only randomized controlled studies are considered ${ }^{143}$.

In the late 1960s, Kamiya ${ }^{144}$ first observed that individuals could gain a degree of voluntary control over the production of alpha activity in their own brain. Almost 25 years later, informed by insights from studies in cognitive neuroscience showing that frontal alpha asymmetry is composed of a trait and state component, a frontal alpha asymmetry neurofeedback protocol was introduced by Rosenfeld (ALAY) ${ }^{145}$. The aim of the protocol is to bias emotional processing via increasing activity in the left hemisphere (e.g., enhance the F4-F3 asymmetry score). Frontal alpha asymmetry neurofeedback has been applied in depressive patients ${ }^{146-150}$, yet these studies were conducted with small samples and no randomized controlled design. Investigating the effect of neurofeedback in healthy participants allow the investigation of protocol specific effects on the electrophysiological level and non-specific effects via the comparison with a placebo or a training group in the undesired direction. Only a few studies looked at the effectiveness of neurofeedback training to change frontal asymmetry and mood state in healthy participants (see table 1). Allen and colleagues ${ }^{151}$ found that a five-day training to relative right-sided frontal activity was associated with less approach motivational experiences to happy movies in healthy participants. Moreover, Harmon-Jones and colleagues ${ }^{152}$ found a specific difference between the increase and decrease frontal asymmetry group on frontal but not on parietal electrodes after two days of neurofeedback training. Another study investigated the possibility of changing frontal asymmetry in a single neurofeedback session ${ }^{153}$. This study 
reported that it is feasible to change frontal asymmetry in both directions in healthy participants, but found no effect on emotion. Taken together, these studies suggest that EEG neurofeedback can be used to change frontal alpha asymmetry, but as an intervention method it requires substantiation, for example by the assessment of the stability, its behavioural usefulness, and by including a control condition.

In chapter 5, we assessed the effectiveness and validity of EEG neurofeedback to change frontal alpha asymmetry to target stress resilience. Three protocols were compared to increase relative right-sided frontal alpha asymmetry, to increase relative left-sided frontal asymmetry, and a placebo control direction. The study extends previous studies by including a placebo control condition and follow-up measurements one week and one month later. Furthermore, we developed a neurofeedback protocol that uses individual alpha and real-time eye-corrected and average referenced data to make instrumental condition of asymmetric alpha oscillations more specific.

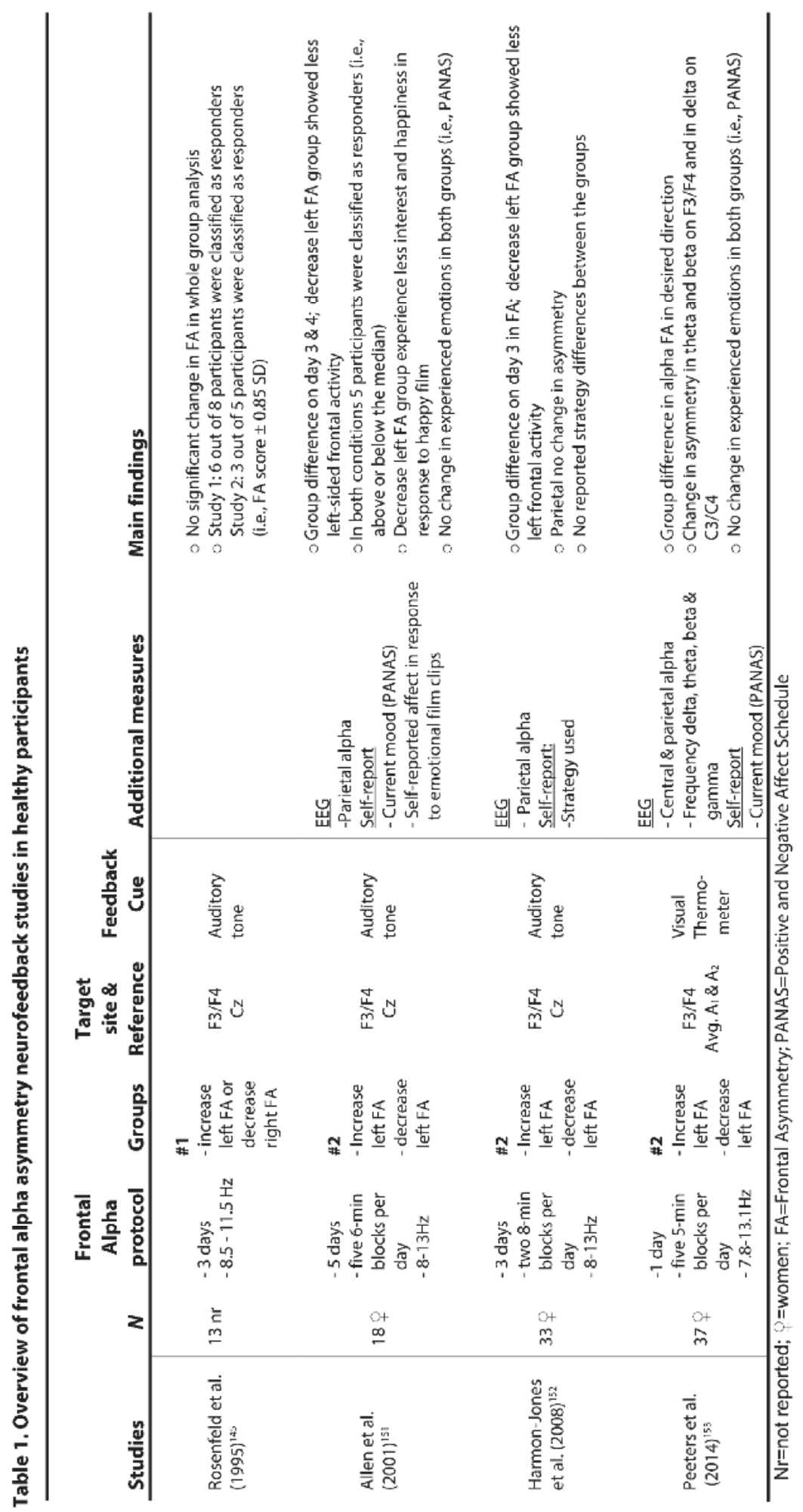




\section{Outline}

This thesis describes studies looking into the neural correlates of acute stress processing in the brain. As described before, inducing stress in a neuroimaging environment can be challenging. Given the prominent role of acute stress induction in this thesis, the first step was to validate the iMAST as a way to induce stress during fMRI scanning (chapter 1). Next, we wanted to assess the effect of task-induced cortisol on the temporal trajectory of functional connectivity of the amygdala, as this is one of the fastest brain areas to react to a stressor. To investigate the time-course of the cortisol effect, healthy participants were studied at rest using resting state fMRI (rs-fMRI), while neuroendocrine stress levels were monitored before, immediately after and $30 \mathrm{~min}$ after stress induction. Based on previous work, it was expected that individual differences in stress responding would be associated with resting state functional connectivity between the amygdala and $\mathrm{mPFC}$, promoting resilience via regulatory feedback on the amygdala (chapter 2 ).

A well-known behavioural effect of stress is its memory enhancing effect on consolidation and its memory impairing effect on retrieval. It has also been suggested that the exact temporal dynamics of stress relative to the memory encoding task are crucial for enhanced consolidation. Thus, we addressed the contribution of cortisol in mediating these effects and determined its neural basis by combining behavioural measures with EEG eventrelated potentials. In order to do so, we applied a stress or a no-stress control version of the MAST either immediately or $30 \mathrm{~min}$ before learning and related the late positive potential (LPP) generated during memory encoding with the performance on the delayed memory test that was carried out $24 \mathrm{~h}$ later (chapter 3 ).

Stress responding and coping is thought to involve lateralized processing in the brain. Moreover, altered lateralized brain activity has been found in stress-related psychopathology. Here, we extended these findings by testing the functional role of frontal alpha asymmetry in stress responding. We conducted an experimental study in healthy men and women and measured frontal alpha lateralisation with EEG at baseline and following exposure to acute stress induced by the MAST. If lateralized processing indeed plays a role in stress responding, it follows that a person's stress resiliency should correlate with frontal asymmetry (chapter 4). Moreover, we were also interested in targeting stress resilience by teaching people to change frontal lateralization with the use of EEG neurofeedback. To test this under laboratory-controlled conditions, we induced acute stress three times using the
MAST and provided a neurofeedback training intervention in-between the first and second stress induction. In a large sample of healthy participants, we compared three frontal asymmetry protocols that aimed to increase relative right- or left-sided frontal asymmetry, relative to a placebo frontal asymmetry control direction. Thus, we examined whether healthy participants were able to learn to change their frontal brain asymmetry using EEG neurofeedback and whether the direction of change influenced task-induced subjective and neuroendocrine stress responses (chapter 5).

The experimental findings and conclusions of the studies presented in this thesis are summarized, discussed, and integrated to form a comprehensive view regarding the neural correlates of stress resilience in the last part of this thesis. Methodological limitations as well as theoretical and clinical implications will be discussed, followed by recommendations for future research. 


\section{References}

1 Bonanno, G.A. (2004). Loss, trauma, and human resilience - Have we underestimated the human capacity to thrive after extremely aversive events? American Psychologist, 59, 20-28.

2 Bonanno, G.A. \& Mancini, A.D. (2008). The human capacity to thrive in the face of potential trauma. Pediatrics, 121, 369 375.

3 Ingram, R.E., \& Luxton, D.D. (2005) Vulnerability-stress models. In B.L. Hankin \& J.R.Z. Abela (Ed.), Development of psychopathology: A vulnerability stress perspective (pp. 32-46). Thousand Oaks, CA: Sage Publications.

4 Dumont, M. \& Provost, M.A. (1999). Resilience in adolescents: Protective role of social support, coping strategies, selfesteem, and social activities on experience of stress and depression. Journal of Youth and Adolescence, 28, 343363.

5 Brewin, C.R., Andrews, B. \& Valentine, J. D. (2000). Meta-analysis of risk factors for posttraumatic stress disorder in traumaexposed adults. Journal of Consulting and Clinical Psychology, 68, 748-766.

6 Gunnar, M. \& Quevedo, K. (2007). The neurobiology of stress and development. Annual Review of Psychology, 58, 145-173.

7 Bakermans-Kranenburg, M.J. \& van IJzendoorn, M.H. (2007). Research Review: Genetic vulnerability or differential susceptibility in child development: the case of attachment Journal of Child Psychology and Psychiatry, 48, 1160-1173.

8 Feder, A., Nestler, E.J. \& Charney, D.S (2009). Psychobiology and molecular genetics of resilience. Nature Reviews Neuroscience, 10, 446-457.

9 Luijk, M.P.C.M. et al. (2010). Attachment, Depression, and Cortisol: Deviant Patterns in Insecure-Resistant and
Disorganized Infants. Developmental Psychobiology, 52, 441-452.

10 Meyer, T., Smeets, T., Giesbrecht, T. \& Merckelbach, H. (2012). The efficiency of reappraisal and expressive suppression in regulating everyday affective experiences. Psychiatry Research, 200 964-969.

11 Rood, L., Roelofs, J., Bogels, S.M. \& Arntz A. (2012). The effects of experimentally induced rumination, positive reappraisal, acceptance, and distancing when thinking about a stressful event on affect states in adolescents. Journal of Abnormal Child Psychology, 40, 73-84.

12 de Kloet, E.R., Joels, M. \& Holsboer, F. (2005). Stress and the brain: from adaptation to disease. Nature Reviews: Neuroscience, 6, 463-475.

13 Ulrich-Lai, Y.M. \& Herman, J.P. (2009) Neural regulation of endocrine and autonomic stress responses. Nature Reviews: Neuroscience, 10, 397-409.

14 McEwen, B.S. (1998). Protective and damaging effects of stress mediators. New England Journal of Medicine, 338 171-179.

15 Simeon, D. et al. (2007). Factors associated with resilience in healthy adults. Psychoneuroendocrinology, 32, 1149-1152.

16 DeRijk, R.H. \& de Kloet, E.R. (2008) Corticosteroid receptor polymorphisms: Determinants of vulnerability and resilience. European Journal of Pharmacology, 583, 303-311.

17 Schwabe, L., Wolf, O.T. \& Oitzl, M.S. (2010). Memory formation under stress: quantity and quality. Neuroscience and Biobehavioral Reviews, 34, 584-591.

18 Smeets, T. (2010). Autonomic and hypothalamic-pituitary-adrenal stress resilience: Impact of cardiac vagal tone. Biological Psychology, 84, 290-295.
19 Zubin, J. \& Spring, B. (1977). Vulnerabilitya new view of schizo-phrenia. Journal of Abnormal Psychology, 86, 103-126.

20 Sullivan, P.F., Neale, M.C. \& Kendler, K.S. (2000). Genetic epidemiology of majo depression: Review and meta-analysis. American Journal of Psychiatry, 157, 1552 1562.

21 Herman, J.P. et al. (2003). Central mechanisms of stress integration: hierarchical circuitry controlling hypothalamo-pituitary-adrenocortical responsiveness. Frontiers in Neuroendocrinology, 24, 151-180.

22 Dickerson, S.S. \& Kemeny, M.E. (2004). Acute stressors and cortisol responses: A theoretical integration and synthesis of laboratory research. Psychological Bulletin, 130, 355-391.

23 Kirschbaum, C., Pirke, K.M. \& Hellhammer, D.H. (1993). The Trier Social Stress Test - A tool for investigating psychobiological stress responses in a laboratory setting Neuropsychobiology, 28, 76-81.

24 Kudielka, B.M., Hellhammer, D.H. \& Kirschbaum, C. (2007). Ten Years of Research with the Trier Social Stress TestRevisited. In E. Harmon-Jones \& P. Winkielman (Eds.), Social neuroscience: Integrating biological and psychological explanations of social behavior (pp. 56-83) New York, NY, US: The Guilford Press.

25 Foley, P. \& Kirschbaum, C. (2010). Human hypothalamus-pituitary-adrenal axis responses to acute psychosocial stress in laboratory settings. Neuroscience and Biobehavioral Reviews, 35, 91-96.

26 Lovallo, W. (1975). The cold pressor test and autonomic function: a review and integration. Psychophysiology, 12, 268282.

27 Porcelli, A.J. et al. (2008). The effects of acute stress on human prefrontal working memory systems. Physiology and Behavior, 95, 282-289.
28 Schwabe, L., Haddad, L. \& Schachinger, H. (2008). HPA axis activation by a socially evaluated cold-pressor test. Psychoneuroendocrinology, 33, 890-895.

29 Smeets, T. et al. (2012). Introducing the Maastricht Acute Stress Test (MAST): A quick and non-invasive approach to elicit robust autonomic and glucocorticoid stress responses. Psychoneuroendocrinology, 37, 1998-2008.

30 Dedovic, K., D'Aguiar, C. \& Pruessner, J.C. (2009). What stress does to your brain: review of neuroimaging studies. Canadian Journal of Psychiatry. Revue Canadienne de Psychiatrie, 54, 6-15.

31 Wang, J. et al. (2005). Perfusion functional MRI reveals cerebral blood flow pattern under psychological stress. Proceedings of the National Academy of Sciences of the United States of America, 102, 17804 17809.

32 Wang, J.J. et al. (2007). Gender difference in neural response to psychological stress. Social Cognitive and Affective Neuroscience, 2, 227-239.

33 Sinha, R., Lacadie, C., Skudlarski, P. \& Wexler, B.E. (2004). Neural circuits underlying emotional distress in humans. Biobehavioral Stress Response: Protective and Damaging Effects, 1032, 254-257.

34 Yang, H.Y. et al. (2007). Gender difference in hemodynamic responses of prefrontal area to emotional stress by near-infrared spectroscopy. Behavioural Brain Research 178, 172-176.

35 Henckens, M.J.A.G., Hermans, E.J., Pu, Z.W., Joels, M. \& Fernandez, G.N. (2009). Stressed Memories: How Acute Stress Affects Memory Formation in Humans. Journal of Neuroscience, 29, 10111-10119.

36 Qin, S.Z., Hermans, E.J., van Marle, H.J.F. Luo, J. \& Fernandez, G. (2009). Acute Psychological Stress Reduces Working Memory-Related Activity in the Dorsolateral Prefrontal Cortex. Biological Psychiatry, 66, 25-32. 
37 van Marle, H.J., Hermans, E.J., Qin, S. \& Fernandez, G. (2009). From specificity to sensitivity: how acute stress affects amygdala processing of biologically salient stimuli. Biological Psychiatry, 66, 649-655.

38 Cousijn, H. et al. (2010). Acute stress modulates genotype effects on amygdala processing in humans. Proceedings of the National Academy of Sciences of the United States of America 107, 9867-9872.

39 Dedovic, K. et al. (2005). The Montreal Imaging Stress Task: using functional imaging to investigate the effects of perceiving and processing psychosocial stress in the human brain. Journal of Psychiatry and Neuroscience, 30, 319-325.

40 Dedovic, K. et al. (2009). Neural correlates of processing stressful information: An event-related fMRI study. Brain Research, 1293, 49-60

41 Pruessner, J.C. et al. (2008). Deactivation of the limbic system during acute psychosocial stress: evidence from positron emission tomography and functional magnetic resonance imaging studies. Biological Psychiatry, 63, 234-240.

42 Dagher, A., Tannenbaum, B., Hayashi, T. Pruessner, J.C. \& McBride, D. (2009). An acute psychosocial stress enhances the neural response to smoking cues. Brain Research, 1293, 40-48.

43 Khalili-Mahani, N., Dedovic, K., Engert, V. Pruessner, M. \& Pruessner, J. C. (2010). Hippocampal Activation During a Cognitive Task Is Associated With Subsequent Neuroendocrine and Cognitive Responses to Psychological Stress. Hippocampus, 20, 323-334.

44 Lederbogen, F. et al. (2011). City living and urban upbringing affect neural social stress processing in humans. Nature, 474 498-501.

45 Lord, C., Steiner, M., Soares, C.N., Carew, C.L. \& Hall, G.B. (2012). Stress response in postpartum women with and without obsessive-compulsive symptoms: an fMRI study. Journal of Psychiatry and Neuroscience, 37, 78-86.

46 Cornelisse, S., Joels, M. \& Smeets, T. (2011). A Randomized Trial on Mineralocorticoid Receptor Blockade in Men: Effects on Stress Responses, Selective Attention, and Memory Neuropsychopharmacology, 36, 27202728.

47 Joels, M. \& de Kloet, E.R. (1994). Mineralocorticoid and glucocorticoid receptors in the brain. Implications for ion permeability and transmitter systems. Progress in Neurobiology, 43, 1-36.

48 Wust, S. et al. (2004). Common polymorphisms in the glucocorticoid receptor gene are associated with adrenocortical responses to psychosocial stress. Journal of Clinical Endocrinology and Metabolism, 89, 565-573.

49 Ter Heegde, F., De Rijk, R.H. \& Vinkers, C.H. (2015). The brain mineralocorticoid receptor and stress resilience. Psychoneuroendocrinology, 52C, 92-110.

50 Herman, J.P. \& Cullinan, W.E. (1997) Neurocircuitry of stress: central control of the hypothalamo-pituitary-adrenocortical axis. Trends in Neurosciences, 20, 78-84.

51 Critchley, H.D. (2005). Neural mechanisms of autonomic, affective, and cognitive integration. Journal of Comparative Neurology, 493, 154-166.

52 Dedovic, K., Duchesne, A., Andrews, J., Engert, V. \& Pruessner, J.C. (2009). The brain and the stress axis: the neural correlates of cortisol regulation in response to stress. Neuroimage, 47, 864 871.

53 Pruessner, J.C. et al. (2010). Stress regulation in the central nervous system evidence from structural and functiona neuroimaging studies in human populations - 2008 Curt Richter Award
Winner. Psychoneuroendocrinology, 35, 179-191.

54 Seeley, W.W. et al. (2007). Dissociable intrinsic connectivity networks for salience processing and executive control. Journal of Neuroscience, 27, 2349 2356.

55 Phillips, M.L., Drevets, W.C., Rauch, S.L. \& Lane, R. (2003). Neurobiology of emotion perception I: The neural basis of norma emotion perception. Biological Psychiatry 54, 504-514.

56 McGaugh, J.L. (2004). The amygdala modulates the consolidation of memories of emotionally arousing experiences. Annual Review of Neuroscience, 27, 1-28.

57 Roozendaal, B., McEwen, B.S. \& Chattarii, S. (2009). Stress, memory and the amygdala. Nature Reviews: Neuroscience, 10, 423-433.

58 Quirk, G.J., Likhtik, E., Pelletier, J.G. \& Pare D. (2003). Stimulation of media prefrontal cortex decreases the responsiveness of central amygdala output neurons. Journal of Neuroscience, 23, 8800-8807.

59 Pezawas, L. et al. (2005). 5-HTTLPR polymorphism impacts human cingulate-amygdala interactions: a genetic susceptibility mechanism for depression. Nature Neuroscience, 8, 828834.

60 Shin, L.M. et al. (2005). A functional magnetic resonance imaging study of amygdala and medial prefrontal cortex responses to overtly presented fearful faces in posttraumatic stress disorder. Archives of General Psychiatry, 62, 273 281.

61 Urry, H.L. et al. (2006). Amygdala and ventromedial prefrontal cortex are inversely coupled during regulation of negative affect and predict the diurna pattern of cortisol secretion among older adults. Journal of Neuroscience, 26, 44154425.

62 Banks, S.J., Eddy, K.T., Angstadt, M. Nathan, P.J. \& Phan, K.L. (2007). Amygdala-frontal connectivity during emotion regulation. Social Cognitive and Affective Neuroscience, 2, 303-312.

63 Gianaros, P.J. et al. (2008). Individual differences in stressor-evoked blood pressure reactivity vary with activation volume, and functional connectivity of the amygdala. Journal of Neuroscience, 28 990-999.

64 Kern, S. et al. (2008). Glucose metabolic changes in the prefrontal cortex are associated with HPA axis response to a psychosocial stressor. Psychoneuroendocrinology, 33, 517-529.

65 Wager, T.D. et al. (2009). Brain mediators of cardiovascular responses to social threat: part I: Reciprocal dorsal and ventral sub-regions of the medial prefrontal cortex and heart-rate reactivity. Neuroimage, 47, 821-835.

66 Shin, L.M. \& Liberzon, I. (2010). The Neurocircuitry of Fear, Stress, and Anxiety Disorders. Neuropsychopharma-cology, 35, 169-191.

67 Peres, J.F. et al. (2011). Police officers under attack: resilience implications of an fMRI study. Journal of Psychiatric Research, 45, 727-734.

68 Fox, M.D. \& Raichle, M.E. (2007). Spontaneous fluctuations in brain activity observed with functional magnetic resonance imaging. Nature Reviews: Neuroscience, 8, 700-711.

69 van Marle, H.J., Hermans, E.J., Qin, S. \& Fernandez, G. (2010). Enhanced resting state connectivity of amygdala in the immediate aftermath of acute psychological stress. Neuroimage, 53 348-354.

70 Veer, I.M. et al. (2011). Beyond acute social stress: increased functional connectivity 
between amygdala and cortical midline structures. Neuroimage, 57, 1534-1541.

71 Joels, M., Fernandez, G. \& Roozendaal, B. (2011). Stress and emotional memory: a matter of timing. Trends in Cognitive Sciences, 15, 280-288.

72 Roozendaal, B. \& McGaugh, J.L. (2011) Memory modulation. Behavioral Neuroscience, 125, 797-824.

73 Jelicic, M., Geraerts, E., Merckelbach, H. \& Guerrieri, R. (2004). Acute stress enhances memory for emotional words, but impairs memory for neutral words. Internationa Journal of Neuroscience, 114, 1343-1351.

74 Smeets, T., Jelicic, M. \& Merckelbach, H. (2006). The effect of acute stress on memory depends on word valence. International Journal of Psychophysiology, 62, 30-37.

75 Payne, J.D. et al. (2007). Stress administered prior to encoding impairs neutral but enhances emotional longterm episodic memories. Learning and Memory, 14, 861-868.

76 McGaugh, J.L. (2000). Stress hormones and amygdala regulate long-term memory storage. International Journal of Psychology, 35, 405-405.

77 McGaugh, J.L., McIntyre, C.K. \& Power, A.E. (2002). Amygdala modulation of memory consolidation: interaction with other brain systems. Neurobiology of Learning and Memory, 78, 539-552.

78 Roozendaal, B., Okuda, S., De Quervain D.J.F. \& McGaligh, J.L. (2006) Glucocorticoids interact with emotioninduced noradrenergic activation in influencing different memory functions. Neuroscience, 138, 901-910.

79 LeDoux, J.E. (2000). Emotion circuits in the brain. Annual Review of Neuroscience, 23, 155-184.

80 Roozendaal, B. (2003). Systems mediating acute glucocorticoid effects on memory consolidation and retrieval. Progress in
Neuropsychopharmacology and Biological Psychiatry, 27, 1213-1223.

81 Cahill, L., Gorski, L. \& Le, K. (2003). Enhanced human memory consolidation with post-learning stress: interaction with the degree of arousal at encoding. Learning and Memory, 10, 270-274.

82 Smeets, T., Otgaar, H., Candel, I. \& Wolf O.T. (2008). True or false? Memory is differentially affected by stress-induced cortisol elevations and sympathetic activity at consolidation and retrieval. Psychoneuroendocrinology, 33, 13781386.

83 Joels, M., Karst, H., DeRijk, R. \& de Kloet E.R. (2008). The coming out of the brain mineralocorticoid receptor. Trends in Neurosciences, 31, 1-7.

84 Hermans, E.J., Henckens, M.J., Joels, M. \& Fernandez, G. (2014). Dynamic adaptation of large-scale brain networks in response to acute stressors. Trends in Neurosciences, 304-314.

85 Schwabe, L., Joels, M., Roozendaal, B., Wolf, O.T. \& Oitzl, M.S. (2012). Stress effects on memory: an update and integration. Neuroscience and Biobehavioral Reviews, 36, 1740-1749.

86 Lovallo, W.R., Robinson, J.L., Glahn, D.C. \& Fox, P.T. (2010). Acute effects of hydrocortisone on the human brain: an fMRI study. Psychoneuroendocrinology 35, 15-20.

87 Joels, M., Pu, Z., Wiegert, O., Oitzl, M.S. \& Krugers, H.J. (2006). Learning under stress: how does it work? Trends in Cognitive Sciences, 10, 152-158.

88 Smeets, T., Giesbrecht, T., Jelicic, M. \& Merckelbach, H. (2007). Contextdependent enhancement of declarative memory performance following acute psychosocial stress. Biological Psychology, $76,116-123$

89 Smeets, T. et al. (2009). Stress selectively and lastingly promotes learning of context-related high arousing information. Psychoneuroendocrinology 34, 1152-1161.

90 Zoladz, P.R. et al. (2011). Pre-learning stress differentially affects long-term memory for emotional words, depending on temporal proximity to the learning experience. Physiology and Behavior, 103, 467-476.

91 Karst, H., Berger, S., Erdmann, G., Schutz G. \& Joels, M. (2010). Metaplasticity of amygdalar responses to the stress hormone corticosterone. Proceedings of the National Academy of Sciences of the United States of America, 107, 1444914454.

92 Diamond, D.M., Campbell, A.M., Park, C.R., Halonen, J. \& Zoladz, P.R. (2007). The temporal dynamics model of emotiona memory processing: a synthesis on the neurobiological basis of stress-induced amnesia, flashbulb and traumatic memories, and the Yerkes-Dodson law. Neural Plasticity, 2007, 60803.

93 Wilding, E.L. \& Ranganath, C. (2012). Electrophysiological Correlates of Episodic Memory Processes. In Steven J. Luck \& Emily S. Kappenman (Eds), The Oxford Handbook of Event-Related Potential Components (pp. 373-396). New York: Oxford University Press Inc.

94 Hajcak, G., Weinberg, A., MacNamara., A, \& Foti, D. (2012). ERPs and the Study of Emotion. In Steven J. Luck \& Emily $\mathrm{S}$. Kappenman (Eds), The Oxford Handbook of Event-Related Potential Components (pp 441-474). New York: Oxford University Press Inc.

95 Sullivan, R.M. \& Gratton, A. (1999). Lateralized effects of medial prefrontal cortex lesions on neuroendocrine and autonomic stress responses in rats. Journal of Neuroscience, 19, 2834-2840.

96 Jankord, R. \& Herman, J.P. (2008). Limbic regulation of hypothalamo-pituitary adrenocortical function during acute and chronic stress. Annals of the New York Academy of Sciences, 1148, 64-73.

97 Davidson, R.J. (2000). Affective style psychopathology, and resilience: Brain mechanisms and plasticity. American Psychologist, 55, 1196-1214.

98 Sullivan, R.M. \& Gratton, A. (2002). Prefrontal cortical regulation of hypothalamic-pituitary-adrenal function in the rat and implications for psychopathology: side matters. Psychoneuroendocrinology, 27, 99-114.

99 Cerqueira, J.J., Almeida, O.F. \& Sousa, N. (2008). The stressed prefrontal cortex Left? Right! Brain, Behavior, and Immunity, 22, 630-638.

100 Baeken, C. et al. (2014). One left dorsolateral prefrontal cortical HF-rTMS session attenuates HPA-system sensitivity to critical feedback in healthy females. Neuropsychologia, 57, 112-121.

101 Sullivan, R.M. (2004). Hemispheric asymmetry in stress processing in rat prefrontal cortex and the role of mesocortical dopamine. Stress-the International Journal on the Biology of Stress, 7, 131-143.

102 Lueken, U. et al. (2009). Altered tonic and phasic cortisol secretion following unilateral stroke. Psychoneuroendocrinology, 34, 402-412.

103 Wittling, W. \& Pfluger, M. (1990) Neuroendocrine hemisphere asymmetries: salivary cortisol secretion during lateralized viewing of emotionrelated and neutral films. Brain and Cognition, 14, 243-265.

104 Heller, W. (1993). Neuropsychological mechanisms of individual differences in emotion, personality, and arousal. Neuropsychology, 7, 476-489.

105 Davidson, R.J. (1998). Affective style and affective disorders: Perspectives from affective neuroscience. Cognition \& Emotion, 12, 307-330. 
106 Tomarken, A.J., Davidson, R.J., Wheeler, R.E. \& Doss, R.C. (1992). Individual Differences in Anterior Brain Asymmetry and Fundamental Dimensions of Emotion. Journal of Personality and Social Psychology, 62, 676-687.

107 Davidson, R.J. (2004). What does the prefrontal cortex "do" in affect: perspectives on frontal EEG asymmetry research. Biological Psychology, 67, 219233.

108 Curtis, W.J. \& Cicchetti, D. (2007). Emotion and resilience: A multilevel investigation of hemispheric electro-encephalogram asymmetry and emotion regulation in maltreated and nonmaltreated children. Development and Psychopathology, 19, 811-840.

109 Meyer, T. et al. (2015). The role of frontal EEG asymmetry in post-traumatic stress disorder. Biological Psychology, 108, 6277.

110 Meyer, T. et al. (2014). Frontal EEG asymmetry as predictor of physiological responses to aversive memories. Psychophysiology, 51, 853-865.

111 Harmon-Jones, E., Gable, P.A. \& Peterson, C.K. (2010). The role of asymmetric frontal cortical activity in emotion-related phenomena: A review and update. Biological Psychology, 84, 451-462.

112 Allen, J.J. \& Cohen, M.X. (2010) Deconstructing the "resting" state: exploring the temporal dynamics of frontal alpha asymmetry as an endophenotype for depression. Frontiers in Human Neuroscience, 4, 232.

113 da Silva, F.H., van Lierop, T.H., Schrijer, C.F. \& van Leeuwen, W. S. (1973). Organization of thalamic and cortical alpha rhythms: spectra and coherences. Electroencephalography and Clinical Neurophysiology, 35, 627-639.

114 Klimesch, W., Sauseng, P. \& Hanslmayr, S. (2007). EEG alpha oscillations: the inhibition-timing hypothesis. Brain Research Reviews, 53, 63-88.

115 Jensen, O. \& Mazaheri, A. (2010). Shaping functional architecture by oscillatory alpha activity: gating by inhibition. Frontiers in Human Neuroscience, 4, 186.

116 Henriques, J.B. \& Davidson, R.J. (1990). Regional brain electrical asymmetries discriminate between previously depressed and healthy control subjects. Journal of Abnormal Psychology, 99, 2231.

117 Thibodeau, R., Jorgensen, R.S. \& Kim, S. (2006). Depression, anxiety, and resting frontal EEG asymmetry: a meta-analytic review. Journal of Abnormal Psychology 115, 715-729.

$118 \mathrm{Kemp}$, A.H. et al. (2010). Disorder specificity despite comorbidity: Resting EEG alpha asymmetry in majo depressive disorder and post-traumatic stress disorder. Biological Psychology, 85, 350-354.

119 Segrave, R.A. et al. (2011). Individualized Alpha Activity and Frontal Asymmetry in Major Depression. Clinical EEG and Neuroscience, 42, 45-52.

120 Nusslock, R. et al. (2011). Cognitive Vulnerability and Frontal Brain Asymmetry: Common Predictors of First Prospective Depressive Episode. Journal of Abnormal Psychology, 120, 497-503.

121 Papousek, I., Reiser, E.M., Weber, B Freudenthaler, H.H. \& Schulter, G. (2012). Frontal brain asymmetry and affective flexibility in an emotional contagion paradigm. Psychophysiology, 49, 489-498.

122 Jackson, D.C. et al. (2003). Now you feel it now you don't: Frontal brain electrica asymmetry and individual differences in emotion regulation. Psychological Science, 14, 612-617.

123 Koslov, K., Mendes, W.B., Pajtas, P.E. \& Pizzagalli, D.A. (2011). Asymmetry in resting intracortical activity as a buffer to social threat. Psychological Science, 22, 641-649.

124 Lewis, R.S., Weekes, N.Y. \& Wang, T.H. (2007). The effect of a naturalistic stressor on frontal EEG asymmetry, stress, and health. Biological Psychology, 75, 239-247.

125 Tops, M. et al. (2005). Acute cortiso administration modulates EEG alpha asymmetry in volunteers: relevance to depression. Biological Psychology, 69, 181-193.

126 Tops, M., van Peer, J.M., Wester, A.E. Wijers, A.A. \& Korf, J. (2006). Statedependent regulation of cortical activity by cortisol: An EEG study. Neuroscience Letters, 404, 39-43.

127 Kudielka, B.M., Hellhammer, D.H. \& Wust, S. (2009). Why do we respond so differently? Reviewing determinants of human salivary cortisol responses to challenge. Psychoneuroendocrinology, 34, 2-18.

128 Gasser, T., Bacher, P. \& Steinberg, H. (1985). Test-retest reliability of spectral parameters of the EEG. Electroencephalography and Clinical Neurophysiology, 60, 312-319.

129 Tomarken, A.J., Davidson, R.J., Wheeler, R.E. \& Kinney, L. (1992). Psychometric Properties of Resting Anterior Eeg Asymmetry - Temporal Stability and Internal Consistency. Psychophysiology 29, 576-592.

130 Klimesch, W., Schimke, H. \& Pfurtscheller G. (1993). Alpha frequency, cognitive load and memory performance. Brain Topography, 5, 241-251.

131 Doppelmayr, M., Klimesch, W., Pachinger, T. \& Ripper, B. (1998). Individual differences in brain dynamics: importan implications for the calculation of eventrelated band power. Biological Cybernetics, 79, 49-57.

132 Bazanova, O.M. \& Vernon, D. (2014). Interpreting EEG alpha activity.
Neuroscience and Biobehavioral Reviews, 44C, 94-110.

133 Klimesch, W. (1999). EEG alpha and theta oscillations reflect cognitive and memory performance: a review and analysis. Brain Research Reviews, 29, 169-195.

134 Weiskopf, N. et al. (2004). Principles of a brain-computer interface $(\mathrm{BCl})$ based on real-time functional magnetic resonance imaging (fMRI). IEEE Transactions on Biomedical Engineering, 51, 966-970.

135 Heinrich, H., Gevensleben, H. \& Strehl, U. (2007). Annotation: Neurofeedback - train your brain to train behaviour. Journal of Child Psychology and Psychiatry, 48, 3-16.

136 Harmon-Jones, E., Gable, P.A. \& Peterson C.K. (2010). The role of asymmetric frontal cortical activity in emotion-related phenomena: a review and update. Biological Psychology, 84, 451-462.

137 Johnston, S.J., Boehm, S.G., Healy, D., Goebel, R. \& Linden, D.E.J. (2010) Neurofeedback: A promising tool for the self-regulation of emotion networks. Neuroimage, 49, 1066-1072.

138 Sulzer, J. et al. (2013). Real-time fMRI neurofeedback: progress and challenges. Neuroimage, 76, 386-399.

139 Huster, R.J., Mokom, Z.N., EnriquezGeppert, S. \& Herrmann, C.S. (2014). Brain-computer interfaces for EEG neurofeedback: peculiarities and solutions. International Journal of Psychophysiology, 91, 36-45.

140 Lofthouse, N., Arnold, L.E., Hersch, S., Hurt, E. \& DeBeus, R. (2012). A Review of Neurofeedback Treatment for Pediatric ADHD. Journal of Attention Disorders, 16 351-372.

141 Lohr, J.M., Meuier, S.A. \& Parker, L.M. (2001). Neurotherapy does not qualify as an empirically supported behaviora treatment for psychological disorders. the Behavior Therapist, 97-104.

142 Arns, M. \& Kenemans, J.L. (2014) Neurofeedback in ADHD and insomnia: 
Vigilance stabilization through sleep spindles and circadian networks. Neuroscience and Biobehavioral Reviews, 44, 183-194.

143 Moriyama, T.S. et al. (2012). EvidenceBased Information on the Clinical Use of Neurofeedback for ADHD. Neurotherapeutics, 9, 588-598.

144 Kamiya, J. (1968). Conscious control of brain waves Psychology Today, 1,56-60.

145 Rosenfeld, J.P., Cha, G., Blair, T. \& Gotlib, I. H. (1995). Operant (biofeedback) contro of left-right frontal alpha power differences: potential neurotherapy for affective disorders. Biofeedback and Self Regulation, 20, 241-258.

46 Baehr, E., Rosenfeld, J.P. \& Baehr, R. (1997). The Clinical Use of An Alpha Asymmetry Protocol in the Neurofeedback Treatment of Depression. Journal of Neurotherapy, 2, 10-23.

147 Baehr, E., Rosenfeld, J.P. \& Baehr, R. (2001). Clinical Use of an Alpha Asymmetry Neurofeedback Protocol in the Treatment of Mood Disorders. Journal of Neurotherapy, 4, 11-18.

148 Hammond, D. C. (2005). Neurofeedback treatment of depression and anxiety. Journal of Adult Development, 12, 131137.

49 Choi, S.W. et al. (2011). Is Alpha Wave Neurofeedback Effective with
Randomized Clinical Trials in Depression? A Pilot Study. Neuropsychobiology, 63, 43-

150 Peeters, F., Oehlen, M., Ronner, J., van Os, . \& Lousberg, R. (2014). Neuro-feedback as a treatment for major depressive disorder-a pilot study. Plos One, 9, e91837.

151 Allen, J.J., Harmon-Jones, E. \& Cavender, J.H. (2001). Manipulation of asymmetry through biofeedback alters self-reported emotional responses and facial EMG. Psychophysiology, 38, 685693.

152 Harmon-Jones, E., Harmon-Jones, C., Fearn, M., Sigelman, J. D. \& Johnson, P. (2008). Left frontal cortical activation and spreading of alternatives: Tests of the action-based model of dissonance. Journal of Personality and Social Psychology, 94, 1-15.

153 Peeters, F., Ronner, J., Bodar, L., van Os, J. \& Lousberg, R. (2014). Validation of a neurofeedback paradigm: manipulating frontal EEG alpha-activity and its impact on mood. International Journal of Psychophysiology, 93, 116-120. 


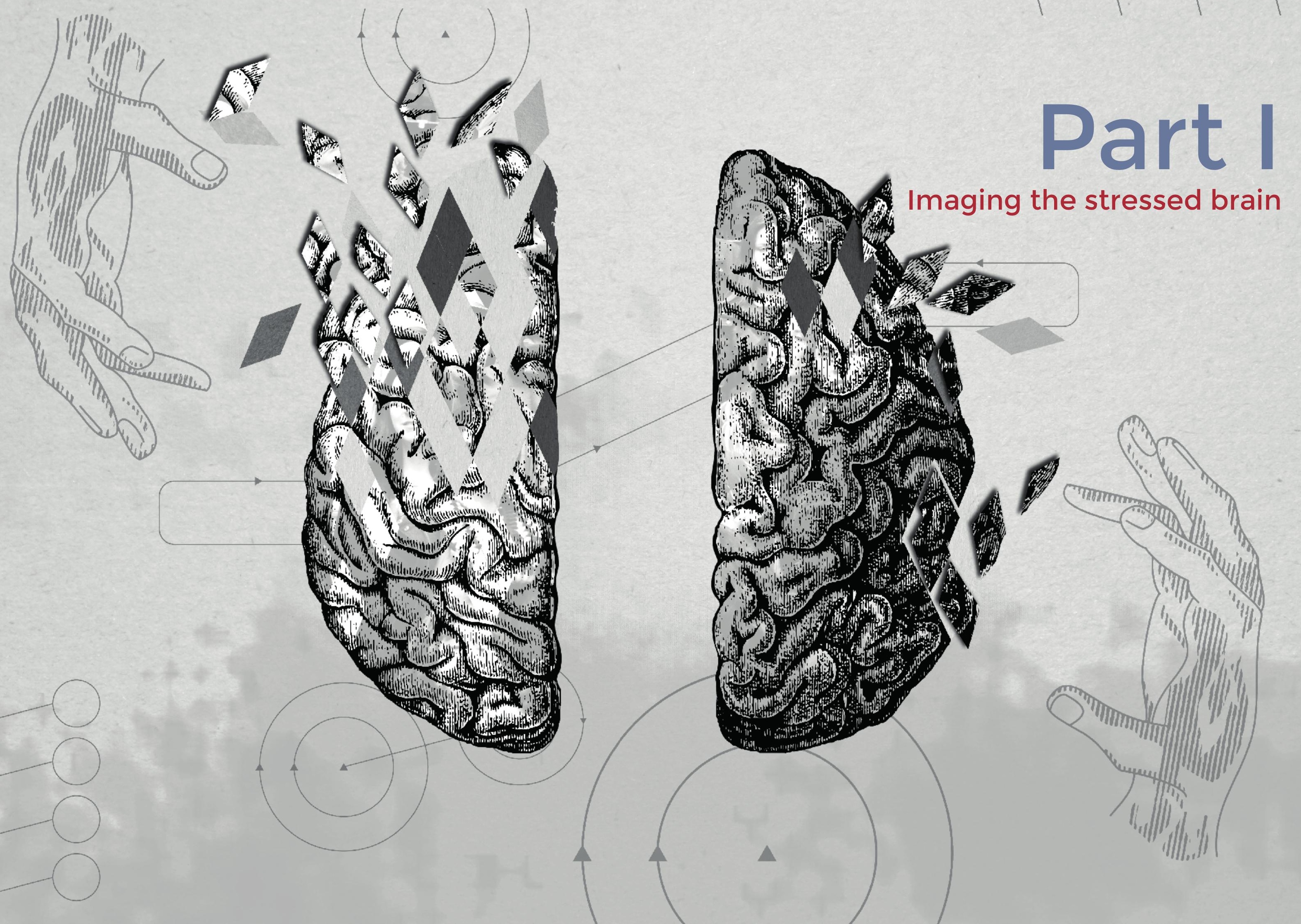




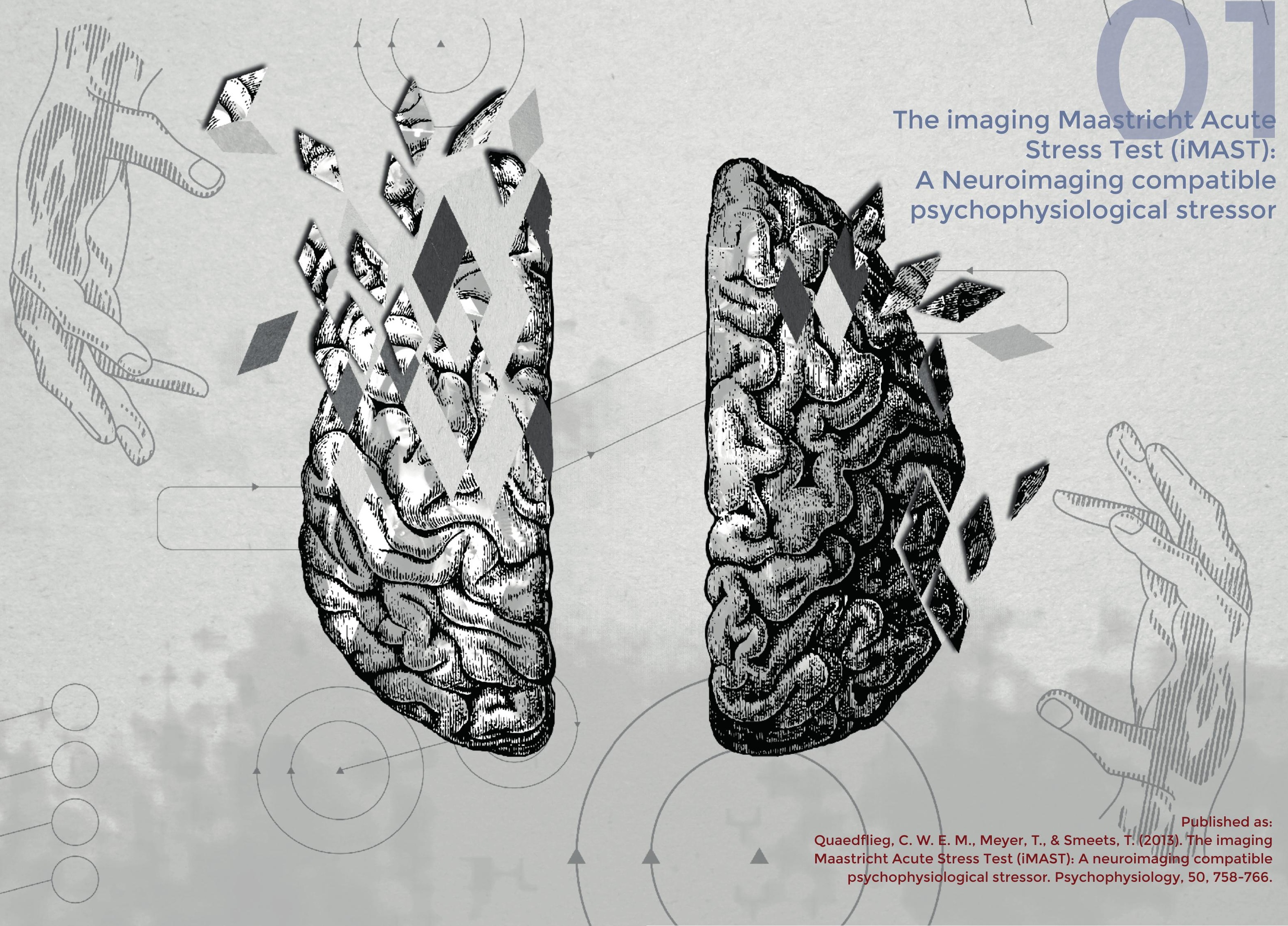




\section{Abstract}

Several protocols have been developed for inducing acute stress in laboratory settings. Still, effectively eliciting stress in a neuroimaging environment remains challenging. Here, we describe the evaluation of a combined physical and psychosocial stress protocol $(n=42)$. The imaging Maastricht Acute Stress Test (iMAST) consists of a 5-min preparation phase and a 10-min acute stress phase, with alternating trials of cold pressor stress generated through an advanced thermal stimulator and mental arithmetic challenges along with socialevaluative pressure (i.e., negative feedback). Results demonstrate that participants displayed meaningful subjective stress responses, as well as significant increases in salivary alpha amylase and cortisol levels. Our data show that the iMAST may prove to be a potent alternative to existing imaging stress paradigms to explore post-stress neuronal changes and brain determinants of resilience.

Keywords: cortisol, functional Magnetic Resonance Imaging (fMRI), Maastricht Acute Stress Test (MAST), stress tasks, stress reactivity 
When a situation is perceived as a physical or psychological threat, the brain activates two physiological systems that jointly enable the individual to cope with the current stressor. The rapidly acting autonomic nervous system (ANS) ensures the release of adrenaline and noradrenaline, which help to potentiate arousal, alertness and focused attention. In turn, the stimulation of the slower hypothalamic-pituitary-adrenal (HPA) axis, following a cascade of events, results in the secretion of the primary human glucocorticoid cortisol'. The contribution of various brain areas in regulating neuroendocrine stress responses depends on the severity and type of stress. Physical stressors (e.g., pain) are known to rapidly activate the ANS and HPA-axis via the brainstem, whereas psychological stressors (e.g., social challenge tests) are mainly processed by the prefrontal cortex (PFC) and engage the limbic system ${ }^{1-3}$.

Numerous protocols have been developed for inducing acute stress in laboratory settings, with the psychosocial Trier Social Stress Test (TSST) ${ }^{4}$ and the physical Cold Pressor Test (CPT) $)^{5,6}$ being used most frequently. The TSST consists of a short preparation period followed by a speech and mental arithmetic task of 5-min each, both performed in front of an audience. In the CPT, participants are instructed to immerse their hand in ice-cold wate for as long as possible for a maximum of 3-min. Both paradigms differ in the degree of ANS and HPA-axis stimulation, which is related to different degrees of uncontrollability, unpredictability and social evaluation². The recently developed Maastricht Acute Stress Test $(\mathrm{MAST})^{7}$ combines a physical (i.e., hand immersion in ice-cold water) and psychological (i.e., mental challenges including psychosocial evaluative threat) stressor, and has been shown to be a concise and valid procedure to elicit robust subjective, ANS, and HPA-axis stress responses.

In contrast to a range of laboratory stress induction procedures, effectively eliciting stress in a functional Magnetic Resonance Imaging (fMRI) environment has proven to be extraordinary challenging. Until now, several psychological stress paradigms have been used, such as the Montreal Imaging Stress Task ((event)MIST ${ }^{8,9}$, a serial subtraction task (i.e., subtracting 13 from a 4-digit number) ${ }^{10,11}$, displaying emotional pictures ${ }^{12,13}$, or showing aversive movies ${ }^{14-18}$. In comparison to non-imaging laboratory stress paradigms, such as the TSST and MAST, the serial subtraction as well as the emotional stimuli paradigms result in relatively modest cortisol increases. Potential reasons for this may be relatively lower social evaluative threat and achievement demands ${ }^{19}$. Moreover, until now, only one physically stressful paradigm, the CPT, has been used in fMRI studies. For example, Porcelli and colleague ${ }^{20}$ investigated the effects of acute physical stress on working memory. Stress was induced by four 30-s hand-immersion trials in a bucket of $4^{\circ} \mathrm{C}$ water. Of course, working with liquids in a scanning environment is cumbersome and far from ideal.

Paramount among the imaging stress tests so far is the (event)MIST ${ }^{8,9}$, which is composed of a series of computerized mental arithmetic tasks with an induced failure algorithm and a social evaluative threat component. The (event)MIST thus incorporates key characteristics of psychological stress paradigms within the constraints of a neuroimaging environment and, compared to a control condition, elicits a significant hormonal (i.e., cortisol) stress response. Nevertheless, cortisol responses to the (event)MIST are significantly smaller than those of non-imaging stress protocols, and there is a large variation between studies applying the (event)MIST in the number of cortisol responders as well as the maximal cortisol increase $\mathrm{e}^{8,9,21-25}$. This variation may be due to the switching between experimental, rest, and control conditions in the (event)MIST, which might not be optimal for the induction of stress in the majority of people.

The current study was set out to develop and evaluate a stress protocol that is effective in eliciting robust cortisol and alpha amylase responses in an fMRI environment, which would provide a reliable means for investigating post-stress neuronal changes. We adapted the MAST ${ }^{7}$ to create an $\mathrm{fMRI}$ compatible physically and psychologically challenging stress test, which is labelled the imaging Maastricht Acute Stress Test (iMAST). Basically, and closely mimicking the MAST, the iMAST consists of a 5-min preparation phase in which task instructions are given and a 10-min acute stress phase. The acute stress phase includes several exposures to cold pressor stress generated with an advanced thermal stimulator (ATS) and various mental arithmetic challenges along with social-evaluative pressure (i.e., negative feedback). We hypothesized that the iMAST would induce subjective stress and would result in significant increases in salivary alpha-amylase and cortisol.

\section{Method}

\section{Participants}

Forty-two right-handed scanner-naïve participants ( 21 men, 21 women, mean age $=21.8$ years, $S D=2.14$; range: $18-35$ ) participated in the current study. All subjects underwent a screening protocol assessing physical and mental health, fMRI aptness, and handedness. 
Participants were excluded when they had a history of psychiatric, neurologic, cardiovascular or neuroendocrine disease, were considered heavy smokers (i.e., more than 15 cigarettes/day), used medication known to affect the ANS or HPA-axis, were regular drug users, or had a body mass index (weight in kg divided by (height in $\mathrm{m}^{2}{ }^{2}$ ) outside the 18-30 range. An additional exclusion criterion was prior experience with the standard (i.e., nonimaging) MAST. Moreover, for women, an extra inclusion criterion was the use of oral contraceptives to reduce variability in cortisol responses related to hormonal alterations throughout the menstrual cycle phase ${ }^{26}$. All types of oral contraceptives were allowed, and the intake phase was not controlled for. Pregnancy or lactation, on the other hand, served as additional exclusion criteria for women. Test protocols were approved by the standing Ethics Committee of the Faculty of Psychology and Neuroscience, Maastricht University. Al participants signed a written informed consent and were given a small monetary reward in return for their participation.

\section{Stress induction equipment and procedures}

An imaging compatible version of the MAST ${ }^{7}$ was used to induce stress in the fMRI scanner The imaging Maastricht Acute Stress Test (iMAST) consists of a 5-min preparation phase in which the task is explained and a 10-min acute stress phase, which includes several exposures to cold pressor stress and various mental arithmetic challenges along with socialevaluative pressure (i.e., negative feedback). Notably, in laboratory studies cold pressor stress is induced by asking participants to submerge their hand in ice-cold water. Yet, this is not suitable for a scanning environment. Therefore, cold pressor stress in the iMAST was generated with an $\mathrm{fMRI}$ compatible $30 \times 30 \mathrm{~mm}$ Medoc Pathway advanced thermal stimulator (ATS) thermode (Medoc Ltd, Ramat Yishai, Israel) placed on the left volar forearm. In total, five cold pressor stress stimuli of $2^{\circ} \mathrm{C}$ were applied with a variable duration of 45,60 or 90 -s. In between the cold pressor trials, participants had to engage in mental arithmetic challenges, that is counting backwards as fast and accurately as possible in steps of 17 starting at 2.043 for 45,60 , or 90 -s (see Fig. 1.1). The participants were equipped with a magnet compatible headset, consisting of earphones and a microphone that was attached to the headphone. The experimenter monitored the counting via the intercom system and whenever participants counted too slow or made a mistake, they received negative feedback via the intercom (i.e., to count faster or start over at 2043). During these mental arithmetic trials, the baseline temperature of the ATS was $25^{\circ} \mathrm{C}$. Additionally, during each of the mental arithmetic trials, one, two or three hot pulses $\left(49^{\circ} \mathrm{C}\right)$ of 10 -s occurred without warning to increase the unpredictability of the task. Moreover, similar to the standard MAST, the iMAST protocol sought to increase uncontrollability and unpredictability by telling participants that the computer would randomly choose the order and duration of the cold pressor and mental arithmetic trials. However, in reality, the duration and order of cold pressor stress stimuli and arithmetic trials was fixed for all participants (see Fig. 1.1). During stress induction, high-resolution T1-weighted structural images of the whole brain were acquired using a 3 Tesla Siemens MR head-only scanner (MAGNETOM Allegra, Siemens Medical Systems, Erlangen, Germany). The imaging data is presented in chapter 2.

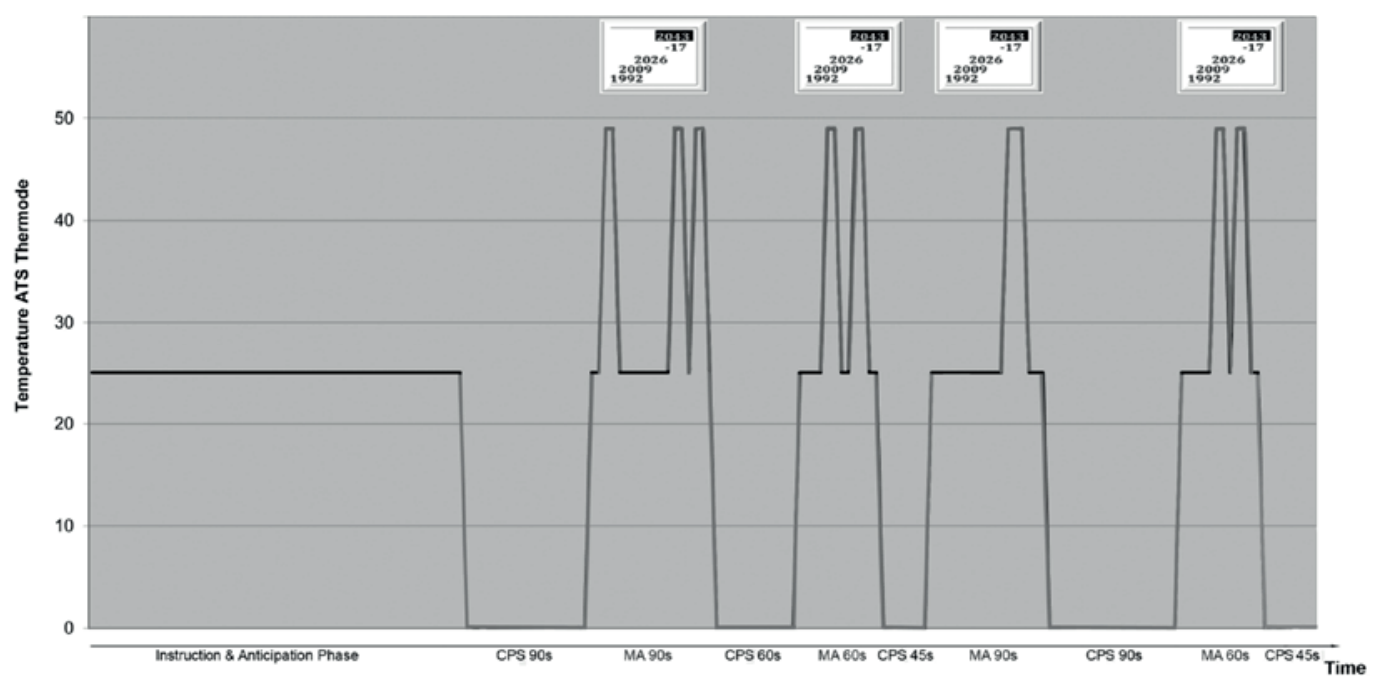

Figure 1.1 Order, duration (in s), and temperature of the cold pressor stress (CPS) and mental arithmetic (MA) of the iMAST.

\section{Subjective and neuroendocrine stress responses}

Subjective stress. Three 100-mm visual analogue scales (VAS) were used to assess subjective stress effects related to the iMAST. After the scanning session had ended, participants had to specify their level of agreement with statements on how stressful, painful and unpleasant they felt during the iMAST by indexing the VAS scales (anchors: $0=$ not at all, $100=$ extremely) 
Salivary alpha-amylase (sAA) and cortisol. Neuroendocrine stress measures prior to and in response to the iMAST were obtained with synthetic Salivette (Sarstedt ${ }^{\circ}$, Etten-Leur, The Netherlands) devices. Participants provided saliva samples immediately after having been placed in the fMRI scanner ( $\left.t_{\text {MRI }}\right)$, 5 -min before the iMAST ( $t_{\text {pre-stressi }}$ i.e., 25 -min after the $t_{M R}$ sample was taken) and four times afterwards $\left(t_{+0,}, t_{+10}, t_{+30}, t_{+40}\right.$ with reference to the end of the stressor). The timing of saliva sampling was based on previous work showing that sAA peaks immediately after stress onset ${ }^{27,28}$, while cortisol typically peaks 20 to 40 -min after stress onset $^{2}$. For each sample collection, participants were returned to the home position of the scanner, and a research assistant then placed the Salivette in the mouth of the participant using sterile plastic tweezers. To facilitate sample collection and to minimize movement, this procedure was practiced during a simulation scan in a dummy MRI device beforehand Saliva samples were stored at $-20^{\circ} \mathrm{C}$ immediately on collection. sAA and cortisol levels were determined from the saliva samples using a commercially available kinetic reaction assay (Salimetrics, Penn State, PA) and luminescence immune assay kit (IBL, Hamburg, Germany), respectively. Mean intra- and inter-assay coefficients of variation are typically less than $8 \%$ and $6 \%$ for the SAA analyses and less than $5 \%$ for both for the cortisol analyses.

\section{Study procedures}

All testing took place between $12: 30$ and 18:00-h to control for the circadian rhythm of cortiso ${ }^{29}$. Participants were asked to refrain from eating, exercising extensively, or drinking anything but non-sparkling water for 2-h prior to the experimental session. After arrival in the laboratory, participants received information on the experimental procedure and gave written informed consent. Next, a saliva sample was taken with the explicit but bogus instruction that it would be immediately assayed to check whether they had adhered to our instructions not to eat, drink, etc. In fact, the sample was destroyed without being analysed. This procedure was followed to promote truth-telling behaviour when participants were subsequently asked whether they had adhered to the instructions. For each measurement, participants were instructed to place the Salivette on the same side of their mouth and not to chew on $\mathrm{it}^{30}$. Next, participants received a standardized lunch (a sandwich and $0.5 \mathrm{I}$ nonsparkling water) during which they were required to fill out several questionnaires (data not reported here). Thereafter and for purposes unrelated to the aims of the current report, electroencephalography (EEG) measurement was prepared and resting EEG activity was measured during 8-min. One and a half hours after arrival, participants were prepared for a 30-min simulation scan to familiarize them with the environment and to reduce potential scanner-induced stress effects ${ }^{31}$. We used a dummy MRI device with exactly the same appearance and noise (transmitted via headphones) as the fMRI scanner in order to mimic the actual fMRI session as closely as possible. Moreover, sAA/cortisol sampling and counting aloud was practiced to minimize head movement during saliva sampling and the IMAST in the scanner. The experimenter observed the participants during training in the dummy scanner and decided whether and when the movement was so insignificant that it was acceptable to start with the actual scanning. Subsequently, participants were placed in the 3 Tesla Siemens MR head-only scanner (MAGNETOM Allegra, Siemens Medical Systems, Erlangen, Germany) and a baseline sAA/cortisol sample ( $\left.\mathrm{t}_{\mathrm{MR}}\right)$ was taken. The fMRI session lasted for about 1.5-h and consisted of the following runs (in chronological order): resting state before stress induction (duration: 8-min), implicit emotion task (16-min), iMAST including anatomical scan (15-min), resting state immediate after stress induction (8-min), emotional working memory task (18-min), and resting state 30-min after stress induction (8min). In total, six saliva samples were collected during scanning (see above). After completion of the scanner session, participants filled in some questionnaires including the VAS items about the iMAST and provided ratings of the stimuli used in the emotional reactivity task (see Fig. 1.2 for an overview of the design). 


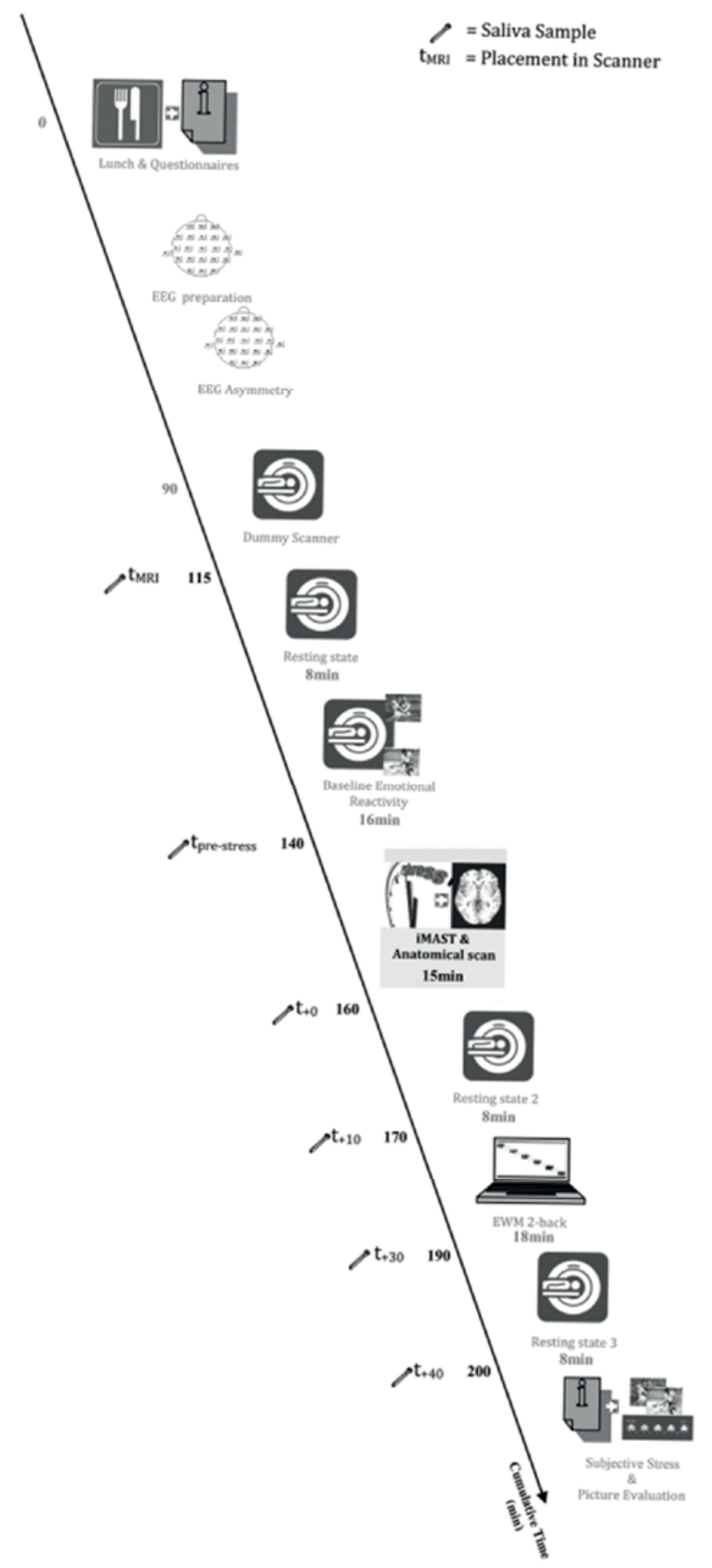

Figure 1.2 Overview of the design and exact timing of the saliva samples.

\section{Data analysis}

Descriptive statistics for subjective stress ratings (VAS scales) are reported and differences in ratings between men and women were analysed using univariate analyses of variance (ANOVAs). Cortisol and sAA data were log-transformed before analyses as Shapiro-Wilk tests of normality showed typical skewness of the data. Cortisol and sAA responses were analysed using a repeated measures ANOVA with Time (6 levels: $\left.t_{M R I}, t_{\text {pre-stress }}, t_{+0}, t_{+10}, t_{+30}, t_{+40}\right)$ as withinsubject factor and Gender as between-subjects factor. For each participant individually, the Area Under the Curve with respect to increase (AUCi) was calculated as a single measure of the total hormone (i.e., SAA and cortisol) concentration in response to the iMAST ${ }^{32}$. The delta increase in cortisol was also computed, defined as peak cortisol concentration after the iMAST minus pre-stress cortisol concentration.

Moreover, a responder rate was calculated representing participants with a cortisol increase equal to or larger than $2.5 \mathrm{nmol} / \mathrm{I}^{4,33}$, which is thought to reflect a cortisol secretory episode ${ }^{34}$. Descriptive statistics for all measures are reported and differences between men and women were analysed using Chi-square tests and univariate ANOVAs. Two men who did not provide enough saliva to be analysed and one clear cortisol outlier (> 3SD above the mean cortisol increase) were excluded from all analyses involving cortisol $(n=39)$. Statistical effects were evaluated using the Greenhouse-Geisser correction and Bonferroni correction when appropriate. $P$-values smaller than .05 were considered statistically significant. In case of significant results, ANOVAs were supplemented with Partial Eta Squared $\left(\eta^{2}{ }_{p}\right)$ values as a measure of effect size $\left(\eta^{2}{ }_{p}\right.$ of 0.01 indicate small effects, $\eta^{2}{ }_{p}$ of 0.06 medium effects, and $\eta^{2}{ }_{p}$ of 0.14 large effects $)^{35}$ and significant follow-up comparisons were supplemented with the standardized mean-change statistic $d$ ( $d$ of 0.20 indicate small effects, $d$ of 0.50 medium effects, and $d$ of 0.80 large effects) $)^{2}$.

\section{Results}

\section{Subjective stress responses}

As shown in Table 1.1, subjects perceived the iMAST as distressing, as indicated by their ratings of subjective stress, pain, and unpleasantness. Men and women did not differ in their subjective reactions to the iMAST, as evidenced by non-significant main effects of gender (stressfulness: $F_{(1,40)}=1.02, p=.32$; painfulness: $F_{(1,40)}=.05, p=.83$ and unpleasantness: $F_{(1,40)}$ $=.09, p=.77)$. 
Table 1.1 Means ( \pm SEM) of subjective stress for men and women participants.

\begin{tabular}{c|ll}
\hline & Men $(\boldsymbol{n}=\mathbf{2 1})$ & Women $(\boldsymbol{n}=\mathbf{2 1})$ \\
\hline Subjective stress (0-100) & & \\
Stress & $76.67(4.29)$ & $71.81(4.49)$ \\
Pain & $53.05(6.12)$ & $54.76(4.89)$ \\
Unpleasantness & $71.71(4.75)$ & $75.00(5.02)$ \\
\hline
\end{tabular}

\section{Neuroendocrine stress responses}

Mean salivary sAA/cortisol concentrations prior to and following the iMAST are shown in Fig. 1.3. For the entire sample, ANOVAs pertaining to SAA data did not reveal a significant Time $\mathrm{x}$ Gender interaction (sAA: $F_{(3.69,95.98)}=.81, p=.52$ ), but did yield the anticipated significant main effect of Time $\left(\mathrm{sAA}: F_{(3.71,100.19)}=5.34, p=.001 ; \eta^{2}{ }_{\mathrm{p}}=0.16\right)$. Bonferroni corrected pairwise comparisons between adjacent time points were used to further evaluate the autonomic stress response to the iMAST over time. Comparison of the saliva sample at placement $\left(\mathrm{t}_{\mathrm{MRI}}\right)$ with the sample immediately before the iMAST ( $t_{\text {prestress }}$ ) demonstrated that lying in the scanner did not induce any changes in SAA levels $(p>.99)$. Significant increases in SAA were found between $t_{\text {prestress }}$ and $t_{+0}(p=.007 ; d=0.62)$, followed by a decrease between $t_{+0}$ and $t_{+10}$ $(p=.01 ; d=-0.46)$ only to remain stable afterwards (i.e., between $t_{+10 \text { vs. }} t_{+30}$ and $t_{+30 \text { vs. }} t_{+40}$; both ps $>$.20). The overall total SAA concentration (AUCi) in response to the iMAST was 857.70 (SEM 198.50) (women: $M=686.90$, SEM 141.63; men $M=1099.67$, SEM 438.13). There were no differences in AUCi between men and women $\left(F_{(1,28)}=1.05, p=.31\right)$.

As to the cortisol data for the total sample, ANOVAs did not reveal a significant Time $x$ Gender interaction (cortisol: $F_{(2.30,82.68)}=.20, p=.85$ ), but again did reveal a the expected significant main effect of Time (cortisol: $F_{(2.30,85.04)}=4.94, p=.007 ; \eta^{2}=0.12$ ). Comparison of the saliva sample at placement $\left(t_{\text {MRI }}\right)$ with the sample immediately before the iMAST ( $t_{\text {pre-stress }}$ ) demonstrated that merely being inside the scanner did not result in a cortisol increase ( $p$ > .99). Follow-up tests did show significant increases in cortisol between $t_{\text {pre-stress }}$ and $t_{+0}(p=$ $.029 ; d=0.29)$, followed by a further increase in cortisol between $\mathrm{t}_{+0}$ and $\mathrm{t}_{+10}(p=.004 ; d=$ 0.25 ), only to remain stable afterwards (i.e., between $t_{+10 \text { vs. }} t_{+30}$ and $t_{+30 \text { vs. }} t_{+40}$; both $p s>.20$ ). Furthermore, relative to the pre-stress sample, cortisol remained significantly increased up until $\mathrm{t}_{+40}\left(\mathrm{t}_{\text {pre-stress }}\right.$ Vs. $\mathrm{t}_{+10}, p<.001 ; d=0.61 ; \mathrm{t}_{\text {pre-stress }}$ vs. $\mathrm{t}_{+30}, p=.04 ; d=0.42 ; \mathrm{t}_{\text {pre-stress }}$ Vs. $\mathrm{t}_{+40,}, p>$ .99). The overall total cortisol concentration (AUCi) in response to the iMAST was 112.92 (SEM 22.90) (women: $M=104.46$, SEM 29.78); men $M=120.99$, SEM 35.19). There were no differences in AUCi between men and women $\left(F_{(1,40)}=0.13, p=.72\right)$.

We then proceeded by calculating a responder rate, representing participants with a cortisol increase equal to or larger than $2.5 \mathrm{nmol} / \mathrm{l}$, which is thought to reflect a cortisol secretory episode. The percentage of participants who could be classified as cortisol responders was $61.5 \%$ (women: $M=70 \%$; men $M=53 \%)$ and did not differ by gender ( $X^{2}(1$, $N=39)=1.24, p=.27)$.

Delta increases in cortisol, i.e., peak cortisol concentration after the iMAST minus prestress cortisol concentration, were also computed. The overall mean cortisol increase (i.e. delta cortisol) was $3.78 \mathrm{nmol} / \mathrm{l}$ (SEM 0.64) (women: $M=3.10$, SEM 0.65; men: $M=4.47$, SEM 1.10). For the cortisol responder group, the mean cortisol increase was $7.90 \mathrm{nmol} / \mathrm{I}$ (SEM 1.30) for men while the mean increase for women was $4.54 \mathrm{nmol} / \mathrm{I}$ (SEM 0.60). For the cortisol responder group, univariate ANOVA on delta cortisol responses indicated a main effect of gender $\left(F_{(1,21)}=6.45, p=.02 \eta_{p}^{2}=0.24\right)$. In contrast, no gender differences with respect to delta cortisol increase were found among the cortisol non-responders $\left(F_{(1,13)}=1.21, p=.29\right.$ delta cortisol women: $M=-.02$, SEM 0.38; men: $M=.66$, SEM 0.43). 
A

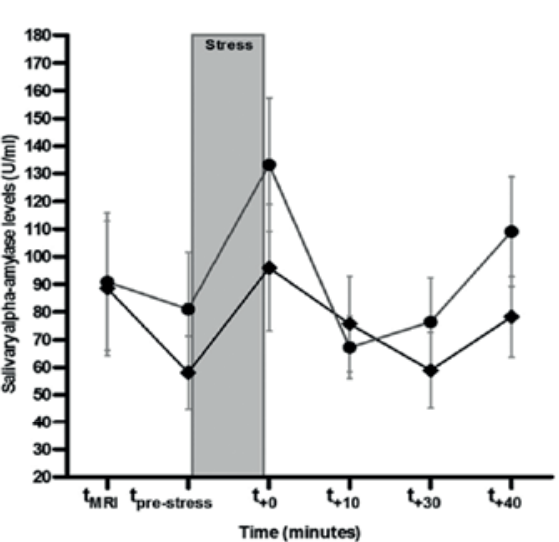

B

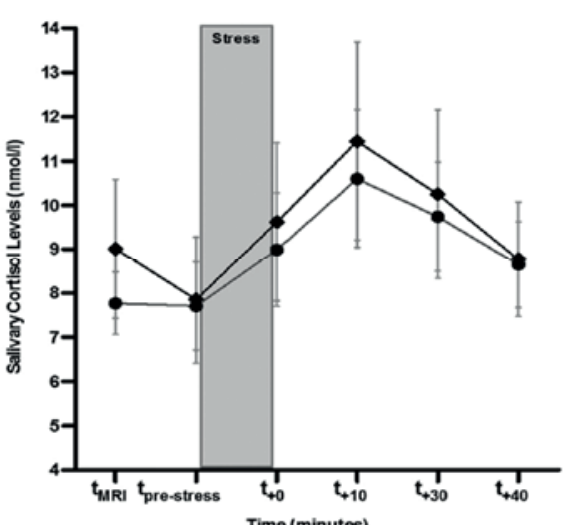

C

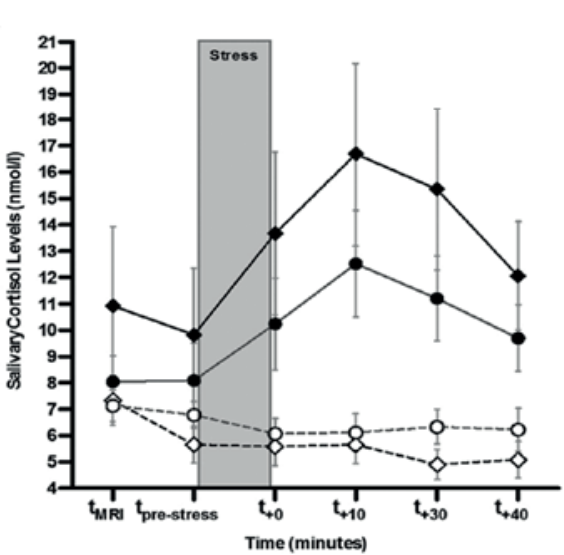

Figure 1.3 Salivary alpha-amylase and cortisol responses to the imaging Maastricht Acute Stress Test (iMAST) for men and women (Panel A \& B), and for men and women cortisol responders and nonresponders separately (Panel C). Values represent (untransformed) Means \pm SEM.

$\mathrm{t}_{\mathrm{MR}}=$ placement in scanner; $\mathrm{t}_{\text {pre-stress }}=$ immediately before the iMAST

\section{Discussion}

The primary aim of the current study was to evaluate the effectiveness of the iMAST as a stress induction paradigm that is capable of eliciting subjective, autonomic, and HPA-axis stress responses within the constraints of a neuroimaging environment. Results demonstrate that the iMAST succeeded in generating considerable subjective stress responses in terms of stressfulness, painfulness, and unpleasantness, as well as robust increases in SAA and cortisol levels in the majority of participants.

The iMAST is the first neuroimaging-suited stress task that relies on a procedure comparable to classic behavioural laboratory stress tasks and thus is characterized by unpredictability and uncontrollability. Additionally, it includes a physical component designed to elicit an immediate bodily reaction via reflexive mechanisms in brainstem and hypothalamus, resulting in a rapid activation of the autonomic nervous system and HPAaxis'. Moreover, the explicit monitoring of and verbal negative feedback on participants' performance while in the scanner creates a social evaluative threat component that triggers stress responses of the HPA-axis, mediated by an activation of the PFC, thalamus, and limbic structures ${ }^{1,3}$. Thus, similar to other laboratory stress tasks, the iMAST follows a double route to stimulate the HPA-axis, which makes it possible to evaluate the contribution of different brain areas in post-stress regulation of cognitive and physiological processes.

Further attesting to the effectiveness of the iMAST in activating the HPA-axis, we found that $61.5 \%$ of all participants displayed cortisol increases larger than $2.5 \mathrm{nmol} /$ (indicative of a cortisol secretory episode) ${ }^{34}$, and could be reliably classified as cortisol responders. Note that this is a more conservative and strict definition of what counts as a cortisol responder, compared to previous neuroimaging studies that employed a post-hoc responder classification of participants who displayed cortisol increases that were much smaller than $2.5 \mathrm{nmol} / /^{9,22,23,25,36}$. While still not approximating some of the most potent nonimaging stress tests such as the TSST and MAST, the current results show that the iMAST is capable of generating significant cortisol responses that are comparable to some other nonimaging laboratory stress tests (e.g., CPT, SECPT) ${ }^{7,37-39}$ and may even exceed those of the existing neuroimaging-suited stress tasks such as the (event)MIST ${ }^{8,9}$.

Notably, we showed that the fMRI procedure by and of itself did not induce significant SAA or cortisol changes, which may be due to the preparation for the MRI scanning procedure including a practice trial in a dummy MRI device. This then, to some 
extent, disagrees with previous work suggesting that the anticipation of (f)MR measurements can moderately increase subjective $\mathrm{e}^{40, \text { but see41 }}$ and neuroendocrine stress markers ${ }^{31,41,42}$, even when a dummy scan preceded the actual MRI measurements ${ }^{43,44}$. These conflicting findings may be related to differences in the timing of the sampling procedure ${ }^{45}$, population characteristics (e.g., age, gender) or the design (e.g., two separate scanning sessions). Additionally, Lueken and colleagues ${ }^{44}$ proposed that the reassuring influence of dummy scanner training depends on the physical arrangement and instructions given. In order to abolish anticipatory anxiety and familiarize them with the scanning environment, participants in the current study were extensively trained in the dummy scanner. This training session lasted for about 30-min and included general information about fMRI measurements, required participants to touch the outside of the scanner while actually being inside so as to get a feeling of their own position, and simulated the noise of the MRI scanner to abolish anticipatory anxiety.

Note that the current study included a stress, but not a no-stress control condition Admittedly, to further investigate the stress-eliciting effects of the scanner environment ${ }^{31}$, and for future research that aims to investigate the effects of stress on various (e.g. cognitive) tasks more broadly, a no-stress placebo version of the iMAST is needed. Consistent with the placebo version for the MAST', the iMAST's no-stress placebo version could consist of small, relatively neutral (e.g., $35^{\circ} \mathrm{C}$ and $40^{\circ} \mathrm{C}$ ) deviations from the normal body temperature, and a simplified counting task (counting consecutively from 1 to 25 ) without performance feedback. This way, the no-stress placebo version would be similar to the iMAST in terms of the order of trials and its duration, but without eliciting stress reactions.

It is well known that laboratory stressors may generate sex differences in HPA-axis responses, with typically larger increases found in men compared to women $26,46-48$, which was confirmed in the current study. Indeed, psychophysiological studies suggest a role for hormonal activity that is specifically related to the menstrual cycle ${ }^{49}$ and its associated differential activation of subcortical arousal structures that are part of stress response circuitry $^{50}$. For instance, Wang and colleagues ${ }^{11}$ found that stress activated the right prefrontal cortex in men, whereas in women stress activated the ventral striatum, putamen, insula, and cingulate cortex. Notably, these differences were observed in the absence of differences in physiological response between genders. Moreover, Wang and colleagues" showed that after completion of the serial subtraction task, only women displayed activity in the anterior and posterior cingulate cortex, structures that have been implicated in emotional processing and reflection of emotional traits. Both lines of research indicate quantitative and qualitative gender differences in stress regulation and indicate the need for combining neuroimaging, physiological, and behavioural approaches.

Even though the iMAST results in robust subjective and neuroendocrine (e.g., cortisol) stress responses, there may also be some potential drawbacks to using the IMAST to generate stress in an fMRI environment. For instance, the iMAST needs the appropriate additional equipment (e.g., the Medoc Pathway stimulator and ATS thermode). Moreover, the physical component of the iMAST might be too intense for certain samples (i.e., children, clinical groups). On the other hand, by having added this physical component to the iMAST an immediate bodily reaction via reflexive mechanisms implicating the brainstem and hypothalamus ${ }^{1,3}$ was ensured, thus triggering a rapid activation of the ANS and the HPA-axis, which in combination with the psychosocial stress components may have been the reason why the iMAST has proven to be effective in yielding solid stress responses. Also, the iMAST requires meticulous training (e.g., in a dummy scanner) beforehand to reduce or eliminate head movement during the counting test. To this end, we instructed participants not to be concerned with their own audibility and, additionally, foam padding was placed around the subject's head in the head coil. It is worth mentioning here that many other studies also used speech during neuroimaging. For example, research investigating the semantic interference effect uses the picture naming task in which participants have to label pictures aloud. These studies looked specifically at the movement induced by speech, and reported less than 3 $\mathrm{mm}$ head movement $\mathrm{t}^{51-55}$. Besides, rotational and translational movement less than the voxel size can be handled well by motion correction. Although we do not have movement parameters from the dummy training or during the anatomical scan (ADNI), the translational and rotational parameters during the resting state functional runs indicate that all participants moved less than $2 \mathrm{~mm}$. Finally, the current design of the iMAST only allows for investigating the post-stress contribution of different brain areas during stress regulation. That is, other imaging stress tests, such as the MIST and serial subtraction, may be more suitable for investigating neural processes during stress induction. Note, however, that the aim of the current study was to develop and evaluate a stress paradigm that closely resembled standard behavioural laboratory stress tests and procedures (e.g., TSST, MAST) 
that typically investigate effects of stress on cognitive processes by administering a task after stress induction.

A few limitations that are specific to the current study also deserve to be mentioned. First, as is typical for research employing VAS scales to assess subjective stress, ratings were obtained after the completion of the stress induction protocol only. However, as a recent study by Hellhammer and Schubert ${ }^{56}$ showed that subjective stress ratings during but not before or after the stress task are associated with physiological stress parameters, repeatedly assessing subjective stress, especially during the iMAST, could prove to be valuable. Second, with respect to gender differences, it may be important to note that all women used oral contraceptives. We used this inclusion criterion to avoid cortisol response variation related to the female menstrual cycle ${ }^{57}$. However, since the hormonal activity of the female menstrual cycle may modulate the activation of the stress response circuitry ${ }^{50}$, future studies may opt to include women during the various phases of the menstrual cycle, which would provide a unique opportunity to investigate the impact of gender specific hormones. That way, one could also establish whether, in line with laboratory stress paradigms such as the TSST, cortisol responses to the iMAST are comparable for men and naturally cycling women in their late luteal phase ${ }^{49}$.

In sum, the current study demonstrates the value of the iMAST as a concise stress paradigm capable of reliably eliciting strong subjective, autonomic and glucocorticoid stress responses in a neuroimaging environment. Our data show that the IMAST may prove to be a powerful alternative to other paradigms in research that seeks a combination of a physical and psychosocial stressor. The iMAST presents researchers with new opportunities for investigating the effects of combined physical and psychological stressor that may aid in the exploration of the anatomical and functional connectivity of the human brain as determinants of individuals' degree of stress resilience or vulnerability.

\section{References}

1 Ulrich-Lai, Y. M. \& Herman, J.P. (2009). Neural regulation of endocrine and autonomic stress responses. Nature Reviews: Neuroscience, 10, 397-409.

2 Dickerson, S.S. \& Kemeny, M.E. (2004). Acute stressors and cortiso responses: A theoretical integration and synthesis of laboratory research. Psychological Bulletin, 130, 355-391.

3 Herman, J.P. et al. (2003). Central mechanisms of stress integration: hierarchical circuitry controlling hypothalamo-pituitary-adrenocortical responsiveness. Frontiers in Neuroendocrinology, 24, 151-180.

4 Kirschbaum, C., Pirke, K.M. Hellhammer, D.H. (1993). The Trier Social Stress Test - A tool for investigating psychobiological stress responses in a laboratory setting Neuropsychobiology, 28, 76-81.

5 Lovallo, W. (1975). The cold pressor test and autonomic function: a review and integration. Psycho-physiology, 12. 268-282.

6 Mitchell, L.A., MacDonald, R.A.R. \& Brodie, E. E. (2004). Temperature and the cold pressor test. Journal of Pain, 5 , 233-237.

7 Smeets, T. et al. (2012). Introducing the Maastricht Acute Stress Test (MAST): A quick and non-invasive approach to elicit robust autonomic and glucocorticoid stress responses. Psychoneuroendocrinology, 37, 19982008.

8 Dedovic, K. et al. (2005). The Montreal Imaging Stress Task: using functiona imaging to investigate the effects of perceiving and processing psychosocial stress in the human brain. Journal of Psychiatry and Neuroscience, 30, 319-325.
9 Dedovic, K. et al. (2009). Neural correlates of processing stressful information: An event-related fMRI study. Brain Research, 1293, 49-60.

10 Wang, J. et al. (2005). Perfusion functional MRI reveals cerebral blood flow pattern under psychological stress. Proceedings of the National Academy of Sciences of the United States of America, 102, 17804-17809.

11 Wang, J.J. et al. (2007). Gender difference in neural response to psychological stress. Social Cognitive and Affective Neuroscience, 2, 227-239.

12 Sinha, R., Lacadie, C., Skudlarski, P. \& Wexler, B. E. (2004). Neural circuits underlying emotional distress in humans. Biobehavioural Stress Response: Protective and Damaging Effects, 1032, 254-257.

13 Yang, H.Y. et al. (2007). Gender difference in hemodynamic responses of prefrontal area to emotional stress by near-infrared spectroscopy. Behavioural Brain Research, 178, 172-176.

14 Cousijn, H. et al. (2010). Acute stress modulates genotype effects on amygdala processing in humans. Proceedings of the National Academy of Sciences of the United States of America, 107, 9867-9872.

15 Cousijn, H., Rijpkema, M., Qin, S. Z., van Wingen, G.A. \& Fernandez, G. (2012). Phasic deactivation of the medial temporal lobe enables working memory processing under stress. Neuroimage, 59, 1161-1167.

16 Henckens, M.J.A.G., Hermans, E.J., Pu, Z., Joels, M. \& Fernandez, G. (2009). Stressed memories: how acute stress affects memory formation in humans. Journal of Neuroscience, 29, 1011110119 
17 Qin, S.Z., Hermans, E.J., van Marle, H.J.F., Luo, J. \& Fernandez, G. (2009). Acute Psychological Stress Reduces Working Memory-Related Activity in the Dorsolateral Prefrontal Cortex. Biological Psychiatry, 66, 25-32.

18 van Marle, H.J., Hermans, E.J., Qin, S. \& Fernandez, G. (2009). From specificity to sensitivity: how acute stress affects amygdala processing of biologically salient stimuli. Biological Psychiatry, 66, 649-655.

19 Dedovic, K., D'Aguiar, C. \& Pruessner, J.C. (2009). What stress does to your brain: a review of neuroimaging studies. Canadian Journal of Psychiatry. Revue Canadienne de Psychiatrie, 54, 6-15.

20 Porcelli, A.J. et al. (2008). The effects of acute stress on human prefrontal working memory systems. Physiology and Behaviour, 95, 282-289.

21 Dagher, A., Tannenbaum, B., Hayashi, T., Pruessner, J.C. \& McBride, D. (2009). An acute psychosocial stress enhances the neural response to smoking cues. Brain Research, 1293, 40-48.

22 Khalili-Mahani, N., Dedovic, K., Engert, V., Pruessner, M. \& Pruessner, J.C. (2010). Hippocampal Activation During a Cognitive Task Is Associated With Subsequent Neuroendocrine and Cognitive Responses to Psychological Stress. Hippocampus, 20, 323-334.

23 Lederbogen, F. et al. (2011). City living and urban upbringing affect neura social stress processing in humans. Nature, 474, 498-501.

24 Lord, C., Steiner, M., Soares, C.N. Carew, C.L. \& Hall, G.B. (2012). Stress response in postpartum women with and without obsessive-compulsive symptoms: an fMRI study. Journal of Psychiatry and Neuroscience, 37, 78-86.
25 Pruessner, J.C. et al. (2008). Deactivation of the limbic system during acute psychosocial stress: evidence from positron emission tomography and functional magnetic resonance imaging studies. Biological Psychiatry, 63, 234-240.

26 Kudielka, B.M., Hellhammer, D.H. \& Wust, S. (2009). Why do we respond so differently? Reviewing determinants of human salivary cortisol responses to challenge. Psychoneuroendocrinology, 34, 2-18.

27 Rohleder, N., Nater, U.M., Wolf, J. M., Ehlert, U. \& Kirschbaum, C. (2004). Psychosocial stress-induced activation of salivary alpha-amylase An indicator of sympathetic activity? Biobehavioural Stress Response: Protective and Damaging Effects, 1032, 258-263.

28 Nater, U.M. et al. (2005). Human salivary alpha-amylase reactivity in a psychosocial stress paradigm. International Journal of Psychophysiology, 55, 333-342.

29 Nicolson, N.A. Mesurement of cortisol. Book Chapter 3.

30 Beltzer, E.K. et al. (2010). Salivary flow and alpha-amylase: Collection technique, duration, and oral fluid type. Physiology and Behaviour, 101, 289-296.

31 Muehlhan, M., Lueken, U., Wittchen, H.U. \& Kirschbaum, C. (2011). The scanner as a stressor: Evidence from subjective and neuroendocrine stress parameters in the time course of a functional magnetic resonance imaging session. International Journal of Psychophysiology, 79, 118-126.

32 Pruessner, J.C., Kirschbaum, C., Meinlschmid, G. \& Hellhammer, D.H. (2003). Two formulas for computation of the area under the curve represent measures of total hormone concentration versus timedependent change. Psychoneuroendocrinology, 28, 916-931.

33 Smeets, T., Jelicic, M. \& Merckelbach H. (2006). The effect of acute stress on memory depends on word valence. International Journal Psychophysiology, 62, 30-37.

34 Van Cauter, E. \& Refetoff, S. (1985). Evidence for two subtypes of Cushing's disease based on the analysis of episodic cortisol secretion. The New England journal of medicine 312, 1343-1349.

35 Fritz, C.O., Morris, P. E. \& Richler, J.J. (2012). Effect Size Estimates: Current Use, Calculations, and Interpretation. Journal of Experimental PsychologyGeneral, 141, 2-18.

36 Soliman, A. et al. (2011). Limbic response to psychosocial stress in schizotypy: A functional magnetic resonance imaging study. Schizophrenia Research, 131, 184-191.

37 Schwabe, L., Haddad, L. \& Schachinger, H. (2008). HPA axis activation by a socially evaluated cold-pressor test. Psychoneuroendocrinology, 33, 890-895.

38 Schwabe, L. \& Wolf, O.T. (2010). Stress impairs the reconsolidation of autobiographical memories. Neurobiology of Learning and Memory, 94, 153-157.

39 Hupbach, A. \& Fieman, R. (2012). Moderate Stress Enhances Immediate and Delayed Retrieval of Educationally Relevant Material in Healthy Young Men. Behavioura Neuroscience, 126, 819-825.

40 Dantendorfer, K. et al. (1997). A study of the effects of patient anxiety, perceptions and equipment on motion artefacts in magnetic resonance imaging. Magnetic Resonance Imaging, 15, 301-306.
41 Tessner, K.D., Walker, E.F., Hochman, K. \& Hamann, S. (2006). Cortisol responses of healthy volunteers undergoing magnetic resonance imaging. Human Brain Mapping, 27, 889-895.

42 van Stegeren, A., Rohleder, N., Everaerd, W. \& Wolf, O. T. (2006). Salivary alpha amylase as marker for adrenergic activity during stress: Effect of betablockade. Psychoneuroendocrinology, 31, 137-141.

43 Eatough, E.M., Shirtcliff, E.A., Hanson, J. L. \& Pollak, S. D. (2009). Hormonal reactivity to MRI scanning in adolescents. Psychoneuroendocrinology, 34, 1242-1246.

44 Lueken, U., Muehlhan, M., Evens, R., Wittchen, H.U. \& Kirschbaum, C. (2012). Within and between session changes in subjective and neuroendocrine stress parameters during magnetic resonance imaging: A controlled scanner training study. Psychoneuroendocrinology, 37, 12991308.

45 Kukolja, J., Thiel, C., Wolf, O. \& Fink, G. (2008). Increased cortisol levels in cognitively challenging situations are beneficial in young but not older subjects. Psychopharmacology, 201, 293-304.

46 Kirschbaum, C., Wust, S. \& Hellhammer, D. (1992). Consistent Sex-Differences in Cortisol Responses to Psychological Stress. Psychosomatic Medicine, 54, 648-657.

47 Kudielka, B.M. \& Kirschbaum, C. (2005). Sex differences in HPA axis responses to stress: a review. Biological Psychology, 69, 113-132.

48 Kajantie, E. \& Phillips, D.I.W. (2006). The effects of sex and hormonal status on the physiological response to acute psychosocial stress. 
Psychoneuroendocrinology, 31, 151178.

49 Kirschbaum, C., Kudielka, B.M., Gaab, J., Schommer, N.C. \& Hellhammer, D. H. (1999). Impact of gender, menstrual cycle phase, and oral contraceptives on the activity of the hypothalamus pituitary-adrenal axis. Psychosomatic Medicine, 61, 154-162.

50 Goldstein, J.M., Jerram, M., Abbs, B Whitfield-Gabrieli, S. \& Makris, N. (2010). Sex differences in stress response circuitry activation dependent on female hormonal cycle. Journal of Neuroscience, 30, 431-438.

51 Barch, D. M., Braver, T.S., Sabb, F. W. \& Noll, D. C. (2000). Anterior cingulate and the monitoring of response conflict: Evidence from an fMRI study of overt verb generation. Journal of Cognitive Neuroscience, 12, 298-309.

52 Christoffels, I.K., Formisano, E. \& Schiller, N.O. (2007). Neural correlates of verbal feedback processing: An fMRI study employing overt speech. Human Brain Mapping, 28, 868-879.

53 Christoffels, I.K., van de Ven, V., Waldorp, L.J., Formisano, E. \& Schiller, N. O. (2011). The Sensory Consequences of Speaking: Parametric Neural Cancellation during Speech in Auditory Cortex. Plos One, 6.

54 Kan, I.P. \& Thompson-Schill, S.L. (2004). Effect of name agreement on prefrontal activity during overt and covert picture naming. Cognitive Affective \& Behavioural Neuroscience, 4, 43-57.

55 Shuster, L.I. \& Lemieux, S.K. (2005). An fMRI investigation of covertly and overtly produced mono- and multisyllabic words. Brain and Language, 93, 20-31.
56 Hellhammer, J. \& Schubert, M. (2012). The physiological response to Trier Social Stress Test relates to subjective measures of stress during but not before or after the test. Psychoneuroendocrinology, 37, 119124

57 Kudielka, B.M., Hellhammer, D.H. \& Wüst, S. (2009). Why do we respond so differently? Reviewing determinants of human salivary cortisol responses to challenge. Psychoneuroendocrinology, 34, 2-18. 


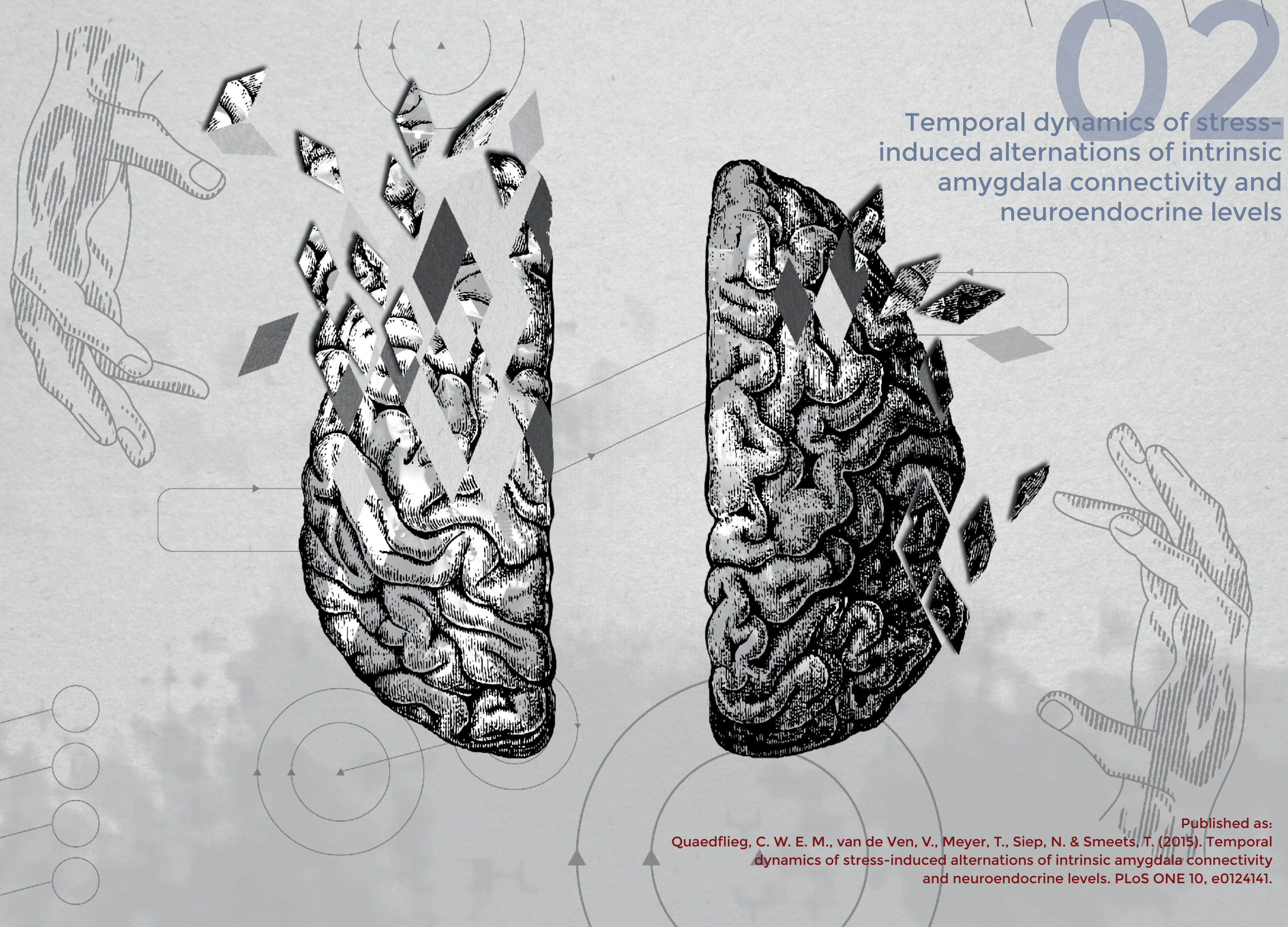




\section{Abstract}

Stress-induced changes in functional brain connectivity have been linked to the aetiology of stress-related disorders. Resting state functional connectivity ( $\mathrm{rsF}$ ) is especially informative in characterizing the temporal trajectory of glucocorticoids during stress adaptation. Using the imaging Maastricht Acute Stress Test (iMAST), we induced acute stress in 39 healthy volunteers and monitored the neuroendocrine stress levels during three runs of resting state functional magnetic resonance imaging (rs-fMRI): before (run 1), immediately following (run 2), and 30-min after acute stress (run 3). The iMAST resulted in strong increases in cortisol levels. Whole-brain analysis revealed that acute stress (run 2 - 1) was characterized by changes in connectivity of the amygdala with the ventrolateral prefrontal cortex (VIPFC), ventral posterior cingulate cortex (PCC), cuneus, parahippocampal gyrus, and culmen. Additionally, cortisol responders were characterized by enhanced amygdala - medial prefrontal cortex (mPFC) connectivity. Stress recovery (run 3 - 2) was characterized by altered amygdala connectivity with the dorsolateral prefrontal cortex (dIPFC), ventral and dorsal anterior cingulate cortex (ACC), anterior hippocampal complex, cuneus, and pre-supplementary motor area (preSMA). Opposite to non-responders, cortisol responders were characterized by enhanced amygdala connectivity with the anterior hippocampal complex and parahippocampal gyrus, and reduced connectivity with left dIPFC, dACC, and culmen during early recovery. Acute stress responding and recovery are thus associated with changes in the functional connectivity of the amygdala network. Our findings show that these changes may be regulated via stress-induced neuroendocrine levels. Defining stress-induced neuronal network changes is pertinent to developing treatments that target abnormal neuronal activity.

Keywords: resting-state functional connectivity, amygdala, acute stress, imaging Maastricht Acute Stress Test (iMAST) 
Dysfunction of neuroendocrine regulation and impaired coping abilities have been implicated in a variety of psychiatric disorders (e.g., depression, anxiety). Acute stress regulated by the neuroendocrine system affects brain activity and, hence, influences the capacity to cope with stress. Glucocorticoids bind to mineralocorticoid and glucocorticoid receptors (MR and GR, respectively) in the brain and exert a time ${ }^{1}$ and spatial ${ }^{2-6}$ specific mode of action, enabling the prioritisation of adaptive cognitive processes after having experienced a stressor. In the immediate phase, stress elicits an emotional response expressed as subjective withdrawal motivation as well as enhanced vigilance, perception and attentional focusing on threat-related stimuli. This phase is focused on promoting survival. Subsequently, processes are initiated directed at restoring homeostasis, such as emotion regulation ${ }^{78}$. To date, only few studies have investigated the activation of distinct brain networks during acute stress and recovery ${ }^{9,10}$ and its relationship to neuroendocrine stress markers ${ }^{11,12}$. This is partly due to fact that it is challenging to effectively elicit neuroendocrine stress responses in the constraints of a neuroimaging environment ${ }^{13}$. The paradigms used until now resulted in relatively modest cortisol increases, making it difficult to address how glucocorticoids change brain activation patterns after a stressor.

The functional connectivity of brain areas has been investigated using task absent (i.e., resting state) functional magnetic resonance imaging measurements (rs-fMRI) ${ }^{14}$. Resting state functional connectivity ( $\mathrm{rSFC}$ ) is especially informative when studying the effects of post-stress brain activation changes. rsFC parameters are not related to a task, making it possible to explore the diffuse effects of stress on the brain. Moreover, connectivity alterations following stress and its relationship to neuroendocrine stress markers are particularly intriguing in light of the suggested role of glucocorticoids in stress adaptation ${ }^{15}$ and the aetiology of stress-related disorders ${ }^{9,10}$.

The amygdala is one of the first brain areas to react to a stressor. It initiates the autonomic nervous system (ANS) and hypothalamic-pituitary-adrenal (HPA) responses, thereby mediating the initial surge in vigilance and optimizing the detection of threats to homeostasis ${ }^{4,7,16}$. Moreover, the amygdala is crucially involved in stress induced long-term adaptive responses such as enhanced memory consolidation ${ }^{17-19}$. The medial prefrontal cortex (mPFC) is involved in mediating amygdala activity during regulation of autonomic and affective responses ${ }^{20-24}$. Previous neuroimaging studies demonstrated time specific enhancement of the functional connectivity of the amygdala with PFC areas during the acute stress ${ }^{9}$ and recovery ${ }^{10}$ phase.

The current study investigated the moderating role of glucocorticoids on the change in amygdala rsFC during two phases that follow stress exposure: the acute and early recovery phases. Stress was induced using the imaging Maastricht Acute Stress Test (iMAST; see also Chapter 1$)^{25}$, a neuroimaging stress task that has been shown to generate considerable subjective stress as well as robust increases in glucocorticoid stress hormones (e.g., the primary human glucocorticoid cortisol). Based on previous studies investigating inter-individual differences in stress reactivity ${ }^{9,26,27}$, we also compared cortisol responders and non-responders in their change of amygdala $\mathrm{rSFC}$ and its relationship to neuroendocrine stress markers. We hypothesized that the acute stress phase is characterized by connectivity changes with areas involved in vigilance and perception, while early recovery is characterized by connectivity changes with areas involved in emotion regulation. Furthermore, amygdala connectivity with the MPFC is hypothesized to differentiate between cortisol responders and non-responders.

\section{Materials and Methods}

\section{Participants}

The sample of the current study consisted of 42 right-handed, scanner-naïve participants (21 men, 21 women, mean age $=21.8$ years, $S D=2.1$; range: $18-35$ years $)^{25}$. All participants underwent a screening protocol assessing their physical and mental health, fMRI aptness, and handedness (see also Chapter 1). Test protocols were approved by the standing ethics committee of the Faculty of Psychology and Neuroscience, Maastricht University. Participants signed a written informed consent form and were given a small monetary reward.

\section{Study procedure}

An overview of the experiment is shown in Fig. 2.1. The three resting state measures were part of a larger study investigating the neural correlates of resilience (see Chapter 1). All testing took place between 12:30 and 18:00-h to control for circadian rhythm of cortisol. One and a half hour after arrival, participants received general information about fMRI measures 
and were prepared for a 30-min simulation scan. To reduce anticipatory anxiety for, and familiarization with the scanner environment, participants were extensively trained in a dummy scanner. Subsequently, participants were placed in the scanner and a baseline cortisol sample $\left(\mathrm{t}_{\mathrm{MRI}}\right)$ was taken. The MRI session lasted for about 1.5-h and consisted of the following runs (in chronological order): resting state before stress induction (duration: 8min), implicit emotion task (16-min), iMAST including anatomical scan (15-min), resting state immediate after stress induction (8-min), emotional working memory task (18-min), and resting state 30-min after stress induction (8-min). During the resting state scan, a fixation point was presented. Participants were instructed to relax, not think of anything in particular and keep their eyes open. In total, six saliva samples were collected during scanning (cf. supra).

The implicit emotion task was used as empirical localizer for left and right amygdala seeds. Participants completed two blocks of the task in which they were asked to decide whether 84 pictures (International Affective Picture System; IAPS) ${ }^{28}$ - subdivided into the categories neutral, positive or negative - were situated in- or outdoors via a button press (see Appendix).

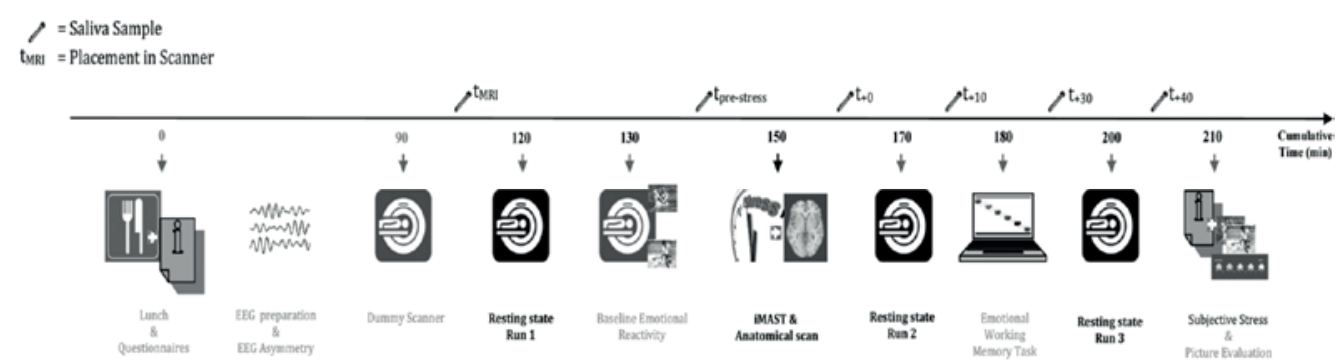

Figure 2.1 Overview of the study procedure. Abbreviation: iMAST: imaging Maastricht Acute Stress Test.

\section{Stress induction equipment and procedures}

The imaging Maastricht Acute Stress Test (iMAST; see Chapter 1$)^{25}$ consists of a 5-min preparation phase in which the task is explained and a 10-min acute stress phase that includes several exposures to cold pressor stress (i.e., stimuli of $2^{\circ} \mathrm{C}$ ) and mental arithmetic challenges (i.e., counting backwards as fast and accurate as possible in steps of 17 starting at 2043) along with social-evaluative pressure (i.e., negative feedback). The iMAST protocol increases unpredictability and uncontrollability by applying without prior warning one, two or three hot pulses $\left(49^{\circ} \mathrm{C}\right)$ of 10 -s during each of the mental arithmetic trials, and by telling participants that the computer would randomly choose the order and duration of the cold pressor and mental arithmetic trials.

\section{Subjective, neuroendocrine and physiological stress responses}

A $100 \mathrm{~mm}$ visual analogue scale (VAS) was used at the end of the imaging session to assess subjective stress related to the iMAST. Participants had to specify their level of agreement with the statement on how stressful they had felt during the IMAST (anchors: $0=$ not at all, $100=$ extremely).

Neuroendocrine stress prior to and in response to the iMAST was measured via saliva samples that were obtained with synthetic Salivettes (Sarstedt, Etten-Leur, The Netherlands). Participants provided saliva samples immediately after having been placed in the MRI scanner ( $\left.\mathrm{t}_{\mathrm{MR}}\right)$, 5 -min before the iMAST ( $\mathrm{t}_{\text {pre-stress }}$ i.e., 25 -min after the $\mathrm{t}_{\text {MRI }}$ sample was taken) and four times after stress exposure $\left(t_{+00}, t_{+10}, t_{+30}, t_{+40}\right.$ with reference to the end of the stressor). For each participant individually, the Area Under the Curve with respect to increase (AUCi) was calculated as a single measure of the total cortisol concentration in response to the iMAST ${ }^{29}$. Two men did not provide enough saliva via the Salivettes to be analysed. One clear cortisol outlier (> 3SD above the mean) was excluded from all analyses. Thus, the final sample consisted of 39 participants.

Based on previous work ${ }^{30}$, delta cortisol increases (i.e., peak cortisol level after the iMAST minus pre-stress cortisol level) of $1.5 \mathrm{nmol} / \mathrm{l}$ or larger were used to distinguish between cortisol responders $(n=27)$ and non-responders $(n=12$, see Fig. 2.2B). The percentage of cortisol responders did not differ by gender $\left(X^{2}(1, N=39)=0.140, p=.71\right)$.

Physiological measurements during MRI scanning included collection of participants' heart rate using a photoplethysmograph placed on the left index finger, and respiratory data using a respiration belt placed around the chest. The physiological data were transformed to power spectral densities (PSD) using Welch's method (pwelch). We investigated whether PSD values differed between the three resting-state measurements of the frequencies that coincided with the resolution of MRI scanning ( $T R=2-5$, resulting in a frequency window-of-interest $0.0075-0.25 \mathrm{~Hz}$ ). 


\section{Data acquisition}

Data were acquired on a 3-T Siemens Magnetom Allegra head scanner (Siemens Medical System, Erlangen, Germany). T1-weighted anatomical images were obtained using an MPRAGE sequence with 192 slices and $1 \times 1 \times 1 \mathrm{~mm}$ voxel size covering the whole-brain (repetition time $(T R)=2250-\mathrm{ms}$, echo time $(T E)=2.6-\mathrm{ms}$, flip angle $\left.=9^{\circ}\right)$. The anatomical images were obtained from each participant during the iMAST. Functional $\mathrm{T} 2^{*}$-weighted resting-state images were acquired using a standard echo-planar imaging (EPI) sequence $\left(\mathrm{TR}=2000-\mathrm{ms}, \mathrm{TE}=30-\mathrm{ms}\right.$, flip angle $=90^{\circ}, 32$ slices, 180 volumes, $\left.3 \times 3 \times 3 \mathrm{~mm}\right)$. Additionally, a negative slice tilt $\left(30^{\circ}\right)$ was used to minimize inhomogeneity artefacts ${ }^{31}$.

\section{fMRI data pre-processing}

The fMRI data were pre-processed and analysed using BrainVoyager QX 2.8 software (Brain Innovation, Maastricht, the Netherlands) ${ }^{32}$. For functional datasets, the first two volumes of each complete time series were discarded because of saturation effects. Pre-processing of the functional data included removing the first two volumes, correction for slice time differences using sinc interpolation, 3D motion correction using sinc interpolation, spatial smoothing using a $4 \mathrm{~mm}$ full-width-at-half-maximum isotropic Gaussian Kernel, and linear trend removal. Individual functional datasets were co-registered with the 3D anatomical data, normalized in Talairach space $(1 \times 1 \times 1 \mathrm{~mm})$, and were averaged to create a group-based mask to exclude voxels belonging to the ventricles or tissue outside of the brain for further analysis.

\section{Selection of amygdala seed from implicit emotion task}

To select amygdala seeds, we investigated the statistical contrast negative $>$ neutral of the implicit emotion task using a whole-brain random effects general linear model (RFX-GLM) The boxcar for the sequences of image presentation was convolved using a two-gamma hemodynamic response function to account for delay of the hemodynamic signal. The resulting contrast map was thresholded using a statistical $(q(F D R)=0.01)$ and cluster-size threshold (i.e. minimum cluster size of $216 \mathrm{~mm}^{3}$ estimated by a stochastic procedure of the statistical map that incorporated the estimated spatial smoothness of the target statistical map with 1,000 Monte Carlo simulations) ${ }^{32}$. We selected a homogeneous voxel cluster in the left (centre coordinate in Talairach space: $x, y, z=-20.82,-5.27,-13.10 ; 253 \mathrm{~mm}^{3}$ ) and the right amygdala $\left(21.73,-6.28,-11.80 ; 305 \mathrm{~mm}^{3}\right)$ as corresponding amygdala seeds (see Fig. 2.3A).

\section{Functional connectivity analysis}

We used seed-based correlation analysis (SCA) to assess amygdala-based whole-brain functional connectivity of the three resting-state measurements. The analysis was performed using NeuroElf (an MR imaging analysis toolbox, www.neuroelf.net) and custom routines in Matlab (Mathworks, Inc.). Data of the left and right amygdala were collapsed because the correlation between the time courses was high for all three resting state runs $(r$ $>.61)$.

We first estimated amygdala-seeded functional connectivity for each participant separately (first-level analysis), which then served as input to a multi-subject statistical analysis (second-level) using an ANOVA model with between-subject factor cortisol responder type and within-subject factor resting state run. Following previous studies and recommendations $\mathrm{s}^{33,34}$, we removed a number of nuisance covariates from the fMRI signal using linear regression before correlating the time series with the amygdala seed. The following parameters were included as regressors: six 3D head motion parameters and their first derivatives, mean signal from the ventricles and white matter, and the global signal. All covariates were Z-normalized to equalize variance. In addition, we added signal oscillations at a frequency above $0.1 \mathrm{~Hz}$ (sine-cosine pairs) for low-pass filtering of the time series. Note that there is a debate as to what the global signal represents ${ }^{35}$, and whether regressing out the global signal is a valid approach ${ }^{36 \text { but see } 37}$. We regressed out the global signal because our focus was on network-specific synchronization of activity unrelated to unspecific global brain activity differences induced by the stressor. After cleaning of the fMRI signal, we then correlated (Pearson's $r$ ) the blood oxygenation level dependent (BOLD) time course extracted from the amygdala seed with the cleaned time series from all other brain voxels for each participant.

The first-level voxel-by-voxel correlation coefficients $r$ were then transformed to normality using Fisher's $Z$ normalization and entered into a second-level analysis to study rsFC changes for acute stress and early recovery using a whole-brain random effects mixedmodel ANOVA. To summarize the data across all participants, we calculated multi-subject maps for each of the resting state measurements using a mass-univariate (i.e., voxel-by- 
voxel) one-sample $t$-test (thresholded for visualization using a statistical $q($ FDR $)=.005$ cluster-size threshold $216 \mathrm{~mm}^{3}$ ). The resulting F-maps were thresholded using a statistical threshold ( $p<.025$, uncorrected) and corrected for multiple comparisons at the 3D cluster level (cluster-level threshold $p=0.05$, estimated minimum cluster-size $=216 \mathrm{~mm}^{3}$ ).

Connectivity changes as a function of time intervals around stress induction were post-hoc analysed using a Bonferroni corrected $t$-test on the differences scores: acute stress (run 2 - run 1) and early recovery (run 3 - run 2). Differences between cortisol responders and non-responders were analysed using a mixed model with cortisol responder type as between-subject factor. Additionally, Pearson's bivariate correlations (Bonferroni corrected, $p<.01)$ were computed to investigate to what extent amygdala rsFC changes were linearly associated with subjective and neuroendocrine stress responses.

\section{Results}

\section{Subjective, neuroendocrine, and physiological stress responses}

We used the iMAST as acute stressor to elicit subjective and neuroendocrine stress responses. Subjects rated the iMAST as distressing on a $100 \mathrm{~mm}$ VAS scale $(M=74.2, S E M=$ 3.24; see Appendix Table S1). There were no differences in subjective stress between cortisol responders and non-responders $\left(F_{(1,37)}=0.32, p=.57\right)$. Neuroendocrine stress responses were defined as salivary cortisol concentration prior to and following the iMAST. For the entire sample, repeated measures ANOVAs revealed significant main effects of Time (6 levels: $\mathrm{t}_{\mathrm{MRI}}$ $\left.\mathrm{t}_{\text {pre-stress, }} \mathrm{t}_{+0}, \mathrm{t}_{+10,} \mathrm{t}_{+30}, \mathrm{t}_{+40 \mathrm{~min}}: F_{(2.30,85.04)}=4.94, p=.007\right)$ with a significant increase of cortisol up until $t_{+40}$ (all $p s<.05$; see Appendix Table S1 \& Fig. 2.2). Comparison of the saliva sample at placement $\left(\mathrm{t}_{\mathrm{MR}}\right)$ with the sample immediately before the iMAST ( $\left.\mathrm{t}_{\text {pre-stress }}\right)$ demonstrated that lying in the scanner did not induce any changes in cortisol $(p>.99 \text {; see also Chapter } 1)^{25}$.

The repeated measures ANOVAs with Time as within-subject factor for pulse and respiratory power spectral densities (PSDs) were non-significant for all frequencies in the window-of-interest ( $p s>0.05$, FWE-corrected) and for the low-frequency range $(<0.1 \mathrm{~Hz}$ pulse: $F_{(2,72)}=1.66, p=0.20$; respiratory: $F_{(2,72)}=1.79, p=0.17$; see Appendix Fig. S1). This indicates that the observed differences between the runs in functional connectivity are not due to differences in physiological responses that are known to influence the BOLD signal in the resting state low-frequency range ${ }^{38,39}$.
A

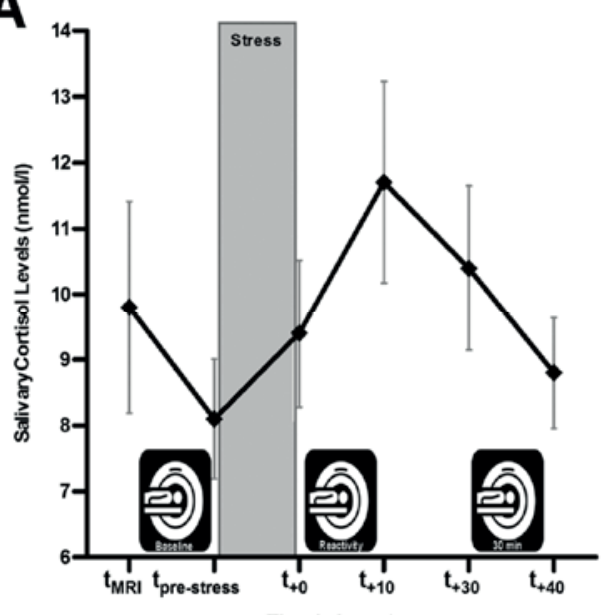

B

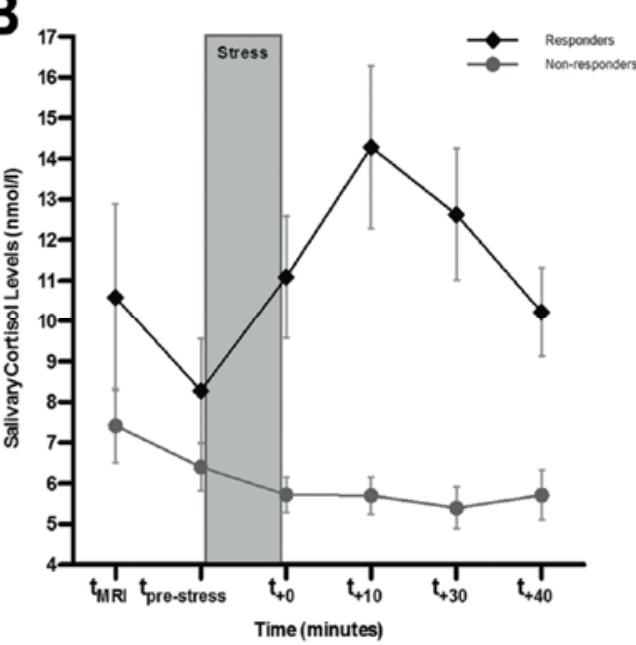

Figure 2.2 Neuroendocrine responses to the imaging Maastricht Acute Stress Test (iMAST). A) Cortisol response with respect to the imaging runs. B) Cortisol responses separately for responders and non-responders. Values represent (untransformed) means \pm SEM.

\section{Amygdala functional connectivity}

We implemented SCA on the three runs of eight minutes of resting state data to investigate how acute stress changes the amygdala connectivity during acute stress and early recovery (see Appendix Fig. S2).

First, brain regions that were functionally coupled to the amygdala were identified for each resting state run separately (one-sample $t$-tests, minimum cluster size of $216 \mathrm{~mm}^{3}$, see Fig. 2.3B). Regions showing significant functional connectivity with the amygdala in all three runs include the lateral frontal pole, lateral orbitofrontal cortex, medial frontal cortex anterior dorsal cingulate cortex, dorsal and ventral posterior cingulate cortex, hippocampus, hypothalamus, insula, temporal pole, superior temporal gyrus, midbrain, and visual cortex. The majority of these regions have been previously described in $\mathrm{rFC}$ of the amygdala ${ }^{10,40,41}$. 
A
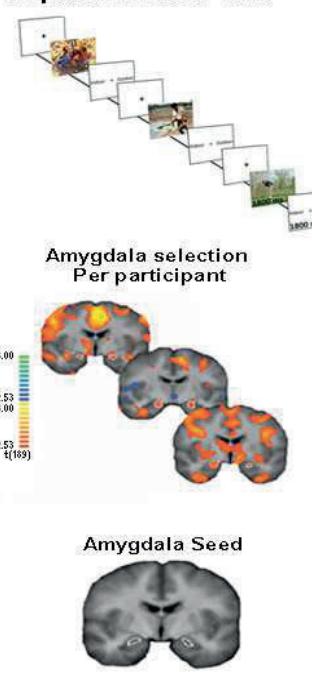
Implicit Emotion Task

B

Amygdala functional connectivity per run
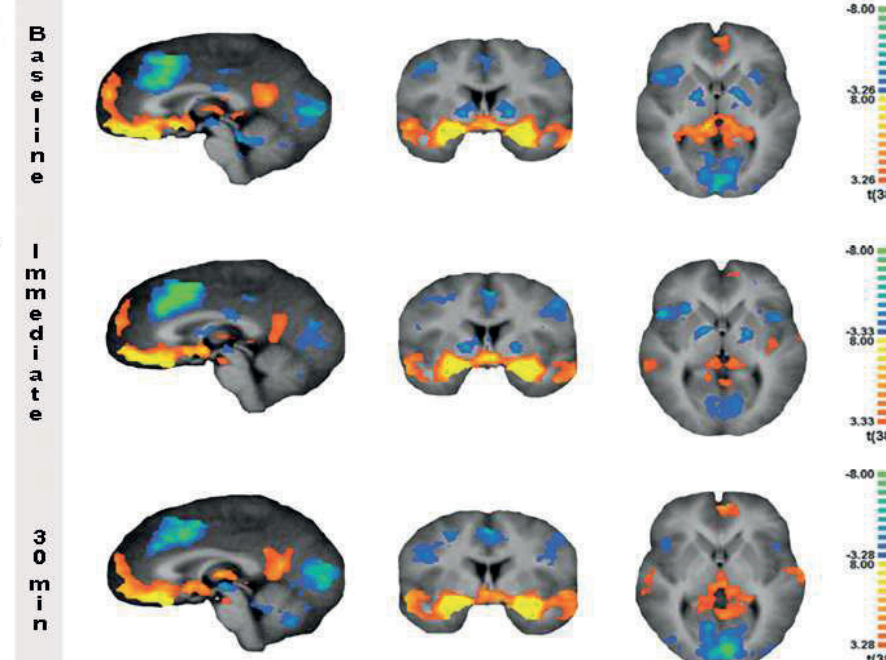

$x=0$
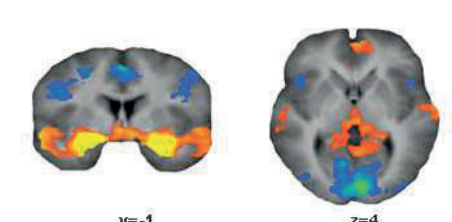

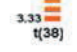

Figure 2.3 Implicit emotion task and functional connectivity of the amygdala. A) The extraction of the amygdala seed. Statistical maps and amygdala ROI selection for the contrast negative > neutral (FDR correction threshold of $q=.001$ ) overlaid on the anatomical average of the participants.

B) Amygdala resting state functional connectivity t-maps per run overlaid on the anatomical average of the participants (FDR correction threshold of $q=.005$ ).

\section{Amygdala functional connectivity during acute stress}

To identify amygdala rsFC changes characterizing acute stress, we subtracted the baseline from the reactivity measurement (run 2 - run 1). A whole-brain analysis revealed that seven clusters showed a significant effect of acute stress during acute stress. A reduced amygdala $\mathrm{rsFC}$ was found with the left ventrolateral prefrontal cortex (VIPFC), left and right ventral posterior cingulate cortex (VPCC), culmen, and bilateral cuneus while the amygdala rsFC was enhanced with the right parahippocampal gyrus immediate after acute stress (see Table 2.1 for statistical values).

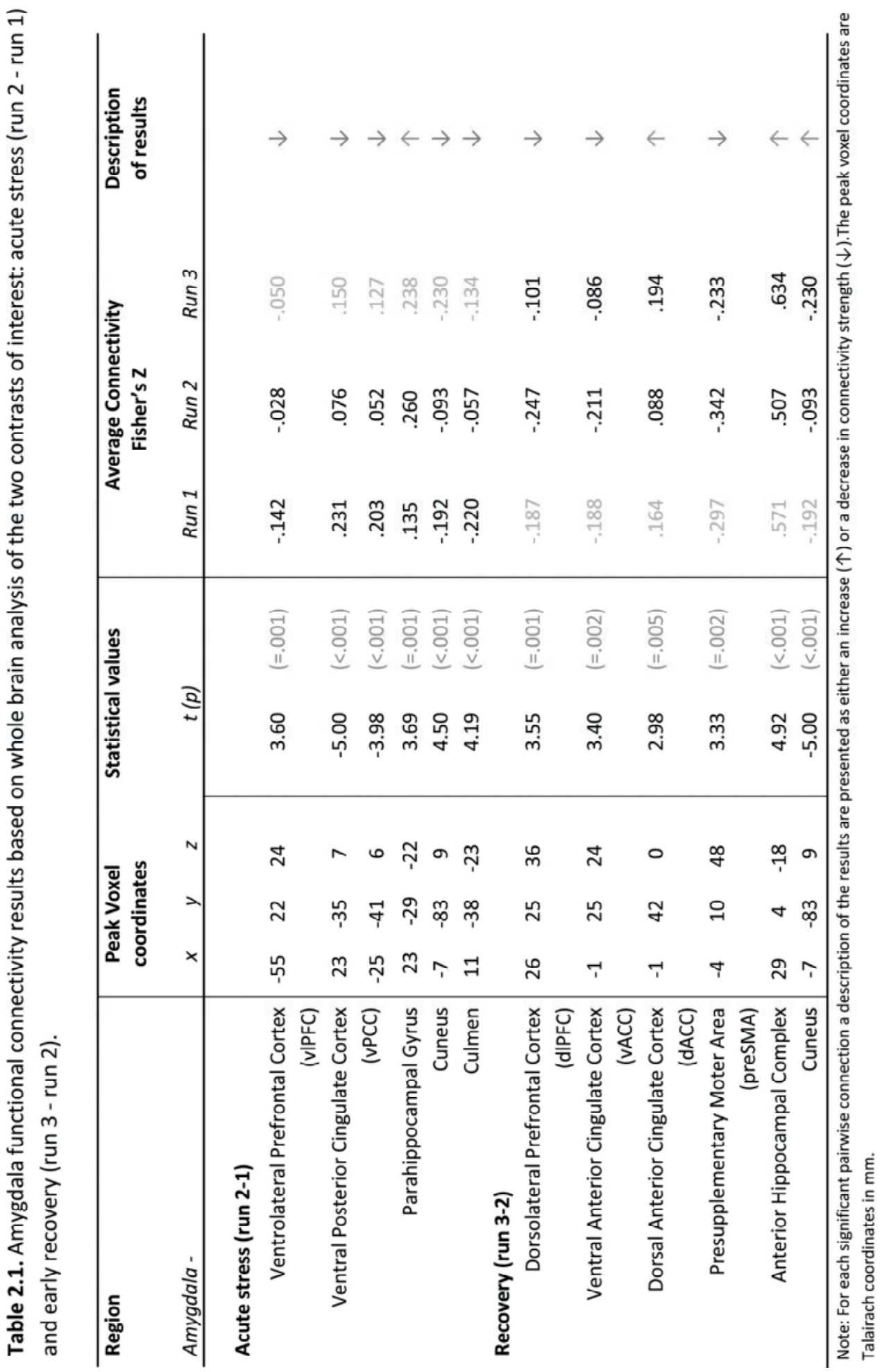


The group contrast of cortisol responders versus non-responders revealed a significant main effect in the mPFC, reflecting an opposing effect of stress on the amygdala rsFC by cortisol responders (enhanced) and non-responders (reduced) (see Fig. 2.4A \& Table 2.2).

A

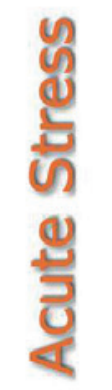

B

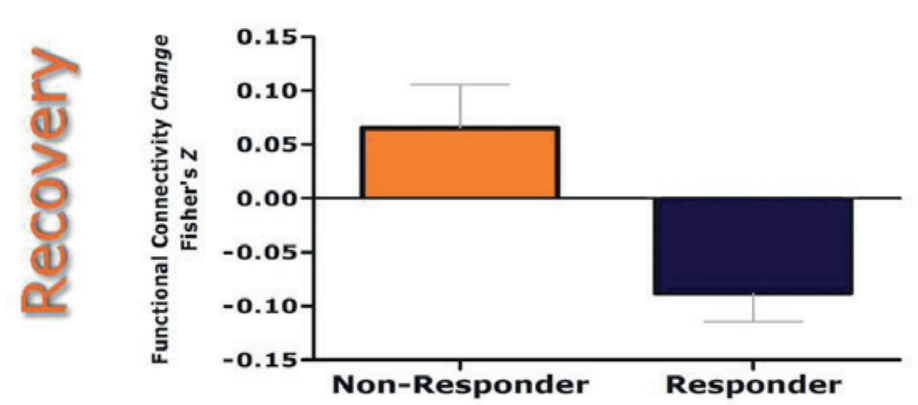

Figure 2.4 Amygdala rsFC differences between cortisol responders and non-responders during the two stress phases. A) Acute stress (run 2 - 1): The group contrast revealed an opposing effect of stress on the amygdala - mPFC rsFC by cortisol responders ( $\uparrow$ ) and non-responders $(\downarrow)$. B) Recovery (run 3-2). The group contrast revealed an opposing effect of stress on the amygdala - dIPFC (shown in the figure), culmen, and AACC rsFC by cortisol responders $(\downarrow)$ and non-responders $(\uparrow)$, while the opposite pattern was found for the rsFC with the anterior hippocampal complex and parahippocampal gyrus responders $(\uparrow)$ and non-responders $(\downarrow)$.

\section{Amygdala functional connectivity during recovery of stress}

To identify amygdala rsFC changes characterizing early recovery of stress, we subtracted the reactivity from the 30-min rs-fMRI run (run 3 - run 2). The whole-brain analysis revealed an effect on the early recovery of acute stress in six clusters. Early acute stress recovery was characterized by reduced amygdala rsFC with the ventral anterior cingulate cortex (vACC), right dorsolateral prefrontal cortex ( $\mathrm{dIPFC}$ ) and the left presupplementary motor area (preSMA) while the amygdala rsFC was enhanced with the dorsal anterior cingulate cortex (dACC), right anterior hippocampal complex consisting of the amygdala and hippocampus, and bilateral cuneus (see Table 2.1).

The group contrast of cortisol responders versus non-responders revealed a significant main effect in five areas reflecting the opposing effect of stress recovery on the amygdala rsFC. Unlike non-responders, cortisol responders were characterized by enhanced amygdala connectivity with the anterior hippocampal complex and parahippocampal gyrus, and reduced connectivity with left dIPFC, dACC, and culmen (see Fig. 2.4B \& Table 2.2 for statistical values).

\begin{tabular}{|c|c|c|c|c|c|c|c|}
\hline Region & \multicolumn{3}{|c|}{$\begin{array}{l}\text { Peak Voxel } \\
\text { Coordinates }\end{array}$} & \multicolumn{2}{|c|}{ Statistical Values } & \multicolumn{2}{|c|}{$\begin{array}{l}\text { Average Connectivity Change } \\
\text { Fisher's } Z \text { (SEM) }\end{array}$} \\
\hline Amygdala - & $x$ & $y$ & $z$ & \multicolumn{2}{|c|}{$t(p)$} & Non-responders & Responders \\
\hline \multicolumn{8}{|l|}{ Acute stress (run 2-1) } \\
\hline $\begin{array}{r}\text { Medial Prefrontal } \\
\text { Cortex (mPFC) }\end{array}$ & -1 & 53 & 15 & -3.35 & $(=.002)$ & $-.130(.04)$ & $.035(.03)$ \\
\hline \multicolumn{8}{|l|}{ Recovery (run 3-2) } \\
\hline $\begin{array}{r}\text { Dorsolateral Prefrontal } \\
\text { Cortex (dIPFC) }\end{array}$ & -34 & 37 & 27 & 3.18 & $(=.003)$ & $.065(.04)$ & $-.088 \quad(.03)$ \\
\hline $\begin{array}{r}\text { Dorsal Anterior Cingulate } \\
\text { Cortex (dACC) }\end{array}$ & -4 & 7 & 39 & 3.36 & $(=.002)$ & $.142(.05)$ & $-.046(.03$ \\
\hline $\begin{array}{r}\text { Anterior Hippocampal } \\
\text { Complex }\end{array}$ & 33 & -29 & -9 & -4.01 & $(<.001)$ & $-.085(.03)$ & $.066(.02$ \\
\hline Parahippocampal Gyrus & -19 & -17 & -15 & -4.31 & $(<.001)$ & $-.077(.04)$ & $.091 \quad(.02)$ \\
\hline Culmen & -17 & -41 & -24 & 4.23 & $(<.001)$ & $.105(.03)$ & $-.076(.02)$ \\
\hline
\end{tabular}




\section{Association of amygdala rsFC with neuroendocrine and subjective measures}

Pearson's bivariate correlations $(p<.01)$ were computed to investigate to what extent amygdala rsFC was linearly associated with total neuroendocrine and subjective stress responses. Baseline (run 1) amygdala - right dmPFC functional connectivity was negatively correlated with the total cortisol concentration AUCi $(r=-.54, p<.001)$. Additionally, amygdala - left dIPFC functional connectivity immediately after stress (run 2) was negatively correlated with subjective stress ( $r=-.49, p=.002$; see Fig. 2.5 ).

\section{Total Cortisol and FC Amygdala - right dmPFC}

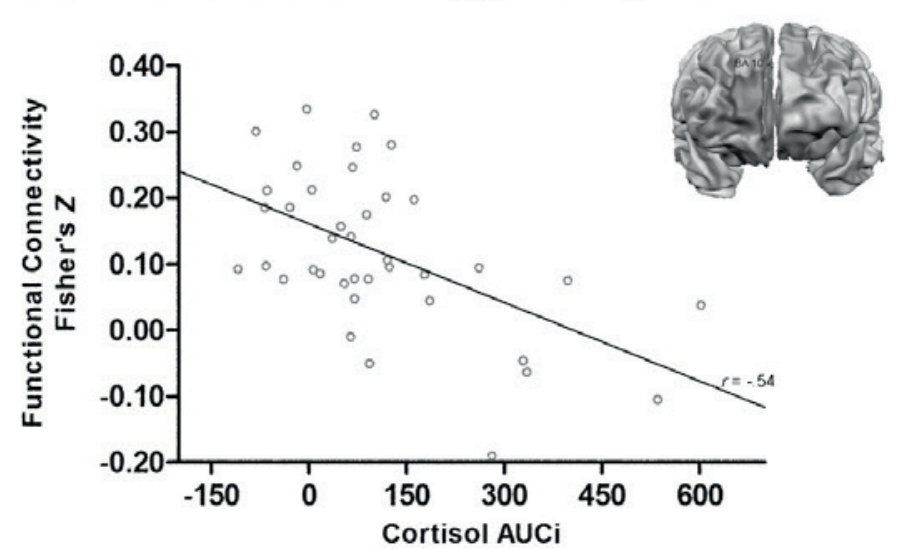

\section{VAS Stress and FC Amygdala - left dIPFC}

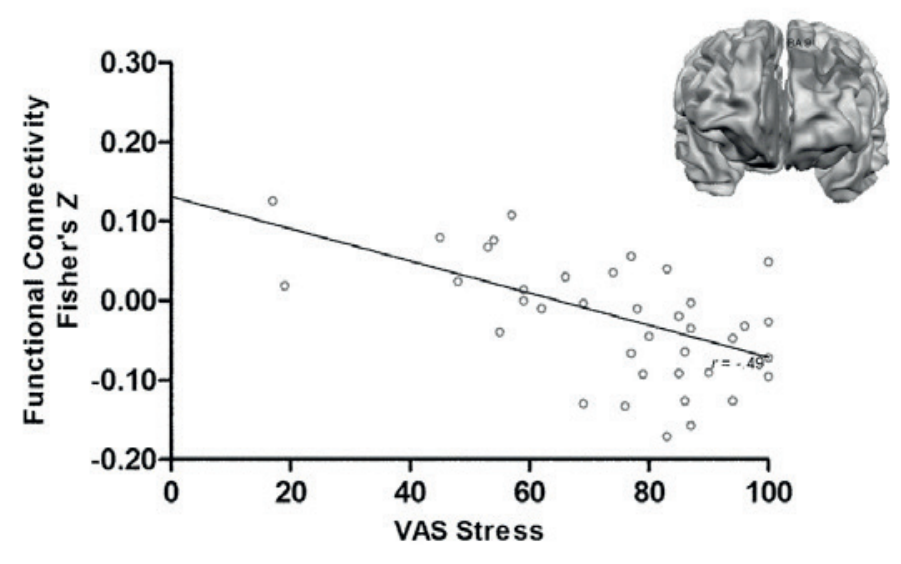

Figure 2.5 Association of amygdala rsFC with total cortisol and subjective stress. The left scatterplot illustrates the negative correlation between total cortisol and strength of the baseline amygdala - right dmPFC connectivity. The right scatterplot illustrates the negative correlation between subjective stress and strength of the reactivity amygdala - left dIPFC connectivity.

\section{Discussion}

The current study investigated how acute stress affects the temporal trajectory of the amygdala's rsFC. Using the iMAST ${ }^{25}$, we obtained strong increases in cortisol levels, enabling us to explore the role of neuroendocrine stress responses in amygdala based rsFC. A particular strength of this study is that it deals with the role of glucocorticoids in the amygdala connectivity changes during two phases that follow stress exposure: acute stress and early recovery.

First, acute stress was characterized by enhanced rsFC between the amygdala and parahippocampal gyrus. The parahippocampal gyrus has a strong functional connection with the amygdala ${ }^{42}$ and has been associated with appraisal and perceived stress ${ }^{43,44}$. Furthermore, the negative rsFC with the vIPFC, cuneus, and culmen and the positive rsFC with the PCC decreased immediately after acute stress. Our results fit with previous reports of increased perfusion in the cuneus at rest immediately after exogenous cortisol administration ${ }^{45}$ as well as enhanced visual processing after acute stress $s^{4,46}$ and during the perception of fear-relevant images ${ }^{47}$ optimizing the detection of threats. In line with this reasoning, we also found decreased PCC connectivity during acute stress. This can be regarded as a sign of deactivation in the default mode network (DMN), which is required for focused attention ${ }^{48}$. Thus, decreased PCC connectivity likely promotes focused attention and optimizes threat detection. Moreover, the decreased amygdala - PCC coupling during acute stress mirrors the findings of Veer and colleagues ${ }^{10}$ of an enhanced coupling during recovery, suggesting a dynamic connectivity pattern modulating threat detection and attention when it is crucial.

Interestingly, during acute stress, the functional connectivity between the amygdala and MPFC was in opposite direction in cortisol responders and non-responders, with an increased connectivity in responders. This finding is seemingly at odds with the absence of a rapid effect of exogenous cortisol administration on the functional connectivity of the amygdala and mPFC during an emotional processing task ${ }^{49}$. One explanation for the disparity may be that exogenous cortisol administration activates almost exclusively the HPA-axis while a psychological stressor also increases the release of other hormones like catecholamines $^{50,51}$. Animal studies have shown that the mPFC activates behavioural and neuroendocrine systems to acute stress ${ }^{52}$. Our results support the notion that the HPA response to stress and stress integrative functions are regulated by forebrain circuits ${ }^{8,53}$. Our 
findings are also in line with previous human studies demonstrating that the mPFC modulates the amygdala response during regulation of autonomic and affective responses ${ }^{20,23,24}$

Second, recovery from the stressor was characterized by a reduced negative connectivity between the amygdala and the ventral ACC and preSMA and enhanced negative rsFC with the cuneus. The VACC is thought to contribute to adaptive emotion regulation, and specifically, to down-regulate limbic regions involved in generating emotional responses ${ }^{54,55}$. In line with this, reduced connectivity between the amygdala and vACC has been found in stress-related psychiatric disorders ${ }^{56,57}$. Additionally, we found that early recovery from the stressor was characterized by changes in connectivity of the amygdala with the $\mathrm{dACC}$ and left dIPFC. These changes in amygdala connectivity interacted with the cortisol response, as evidenced by our cortisol responder versus non-responder analysis. Cortisol responders displayed reduced amygdala - dIPFC and amygdala - dACC functional connectivity. The dACC is part of an intrinsic salience network that regulates adaptive behaviour in response to environmental stimuli that produce autonomic reaction $s^{58-60}$. Our results extend previous reports of enhanced amygdala rsFC with the $\mathrm{dACC}$ during acute stress ${ }^{9,11}$ by directly comparing cortisol responders and non-responders, and by directly comparing the rsFC immediately and 30-min after the stressor. Together, these findings suggest a dynamic connectivity pattern regulating the autonomic response that is dependent on the stress phase. Participants with higher HPA-axis reactivity displayed a reduced amygdala-left dIPFC functional connectivity. Functional asymmetries in the PFC are said to be relevant to stress adaptation, with the left PFC being involved in effective coping and preventing small stressors from becoming significant ones ${ }^{52,61,62}$. Thus, the decreased amygdala - left dIPFC rsFC in cortisol responders suggests less effective coping and possibly increased vulnerability to stress.

Enhanced emotional memory consolidation is a well-known long-term adaptive response after a stressor, and the connectivity between the amygdala and hippocampus is thought to regulate this effect ${ }^{63,64}$. In line with this, we found enhanced connectivity between the amygdala and anterior hippocampal complex during stress recovery. Interestingly, we found that this change in rsFC connectivity was dependent on glucocorticoid levels, as demonstrated by an opposite direction of the functional connectivity between the amygdala and anterior hippocampal complex in cortisol responders and non-responders. In cortisol responders, recovery was characterized by enhanced amygdala rsFC with the left parahippocampal gyrus and right hippocampal complex. This is in accordance with the view that returning glucocorticoids reactivate the hippocampus after initial stress exposure ${ }^{26}$.

Finally, an investigation of the relation between baseline amygdala rsFC and neuroendocrine measure of stress revealed that enhanced amygdala - right dmPFC connectivity was associated with a lower total increase of cortisol in response to the iMAST. This result is consistent with the findings from rodent studies indicating the importance of the dmPFC in reducing HPA activation (for review see ${ }^{52}$ ). Interestingly, subjective stress was inversely associated with amygdala - left dIPFC reactivity rsFC. Immediately after acute stress, stronger amygdala - left dIPFC connectivity was associated with less experienced stress. These findings are in agreement with the idea that the connectivity between the left PFC and amygdala plays a central role in the down-regulation of negative affect ${ }^{65}$ and with the proposed role of asymmetrical activation of the prefrontal cortex in stress adaptation via biased motivational processing.

Several issues deserve consideration. First, in light of the debate regarding a possible influence of tasks on the $\mathrm{rFC}$ in a subsequent resting state measurement ${ }^{66,67}$ but see 68,69 , we investigated the influence of an emotional working memory task on rsFC changes during recovery. Per participant, the time course of the amygdala was extracted in the emotional working memory contrast negative > neutral using a whole-brain random effects general linear model. The individual beta values from the amygdala in the emotional working memory task were then correlated with the individual amygdala rsFC Fisher's $Z$ transformed correlation coefficients $r$ of areas, where a significant difference between run 2 and 3 was found (i.e., dIPFC, ventral and dorsal ACC, presupplementary motor area, anterior hippocampal complex, parahippocampal gyrus, cuneus, and culmen). All correlations were non-significant. Moreover, by using the cortisol responder versus non-responder contrast, the influence of the emotional working memory task was curtailed, because all participants had to perform the task. Second, the current study did not include a no-stress control condition. To further investigate the influence of task-engagement, and for future research aiming to investigate stress mechanisms in the brain, a no-stress placebo version of the iMAST is needed. The no-stress placebo version would be similar to the IMAST in terms of physical and mental load as well as its duration, but without eliciting stress reactions ${ }^{70}$. Third, 
the current study investigated the early recovery phase after stress induction controlled by non-genomic glucocorticoid actions. It would be interesting to also investigate the delayed genomic effect of acute stress on changes in amygdala connectivity by including resting state measurements during a longer period after the stress induction. Glucocorticoids play an important role in both the onset and the termination of the stress-response via the MR and GR. The membrane-bound MR mediates the onset of the stress response while the GR terminates the stress response and facilitates behavioural adaptation ${ }^{7}$. Future studies may extend the current study's test of time-dependent effects of glucocorticoid actions by focusing on the different temporal profiles of the MR and GR (e.g., by pharmacologically blocking the GR or by studying MR polymorphisms that affect the functioning of the MR receptor) and by investigating how these two receptor types mediate the effect of cortisol stress responses on the resting state functional connectivity during the different phases after a stressful experience. Finally, although the amygdala rsFC provides a valuable framework to study the effects of stress, investigating in a more exploratory fashion the temporal dynamics of the entire cortex could yield a comprehensive description of brain functional architecture post-stress.

In sum, this study demonstrates that acute stress and early recovery thereof are associated with changes in the functional connectivity of the amygdala network, which is most probably regulated by stress-induced neuroendocrine levels. The early phase after acute stress is characterized by changes in connectivity strength of the amygdala with areas involved in emotional significance, threat detection, and HPA-axis regulation. The period after removal of the stressor was characterized by connectivity changes in areas involved in emotion regulation, coping, and suppression of negative affect. Dysfunction of the HPA-axis regulation and impaired coping abilities have been implicated in a variety of psychiatric disorders (e.g., depression, anxiety). Defining stress-induced neuronal network changes is highly relevant for developing treatments that target abnormal neuronal activity. Our results suggest that the left dIPFC might be a target area of brain activity based treatments to promote recovery and stress adaptation. Moreover, repeated resting-state assessments may prove valuable for further investigations of intrinsic post-stress brain activation changes so as to further delineate the temporal trajectory during which cortisol affects specific brain connectivity patterns.
1 Joels, M., Karst, H., DeRijk, R. \& de Kloet, E. R. (2008). The coming out of the brain mineralocorticoid receptor. Trends in Neurosciences, 31, 1-7.

2 Hermans, E.J., Henckens, M.J.A.G., Joels, M. \& Fernandez, G. (2014). Dynamic adaptation of large-scale brain networks in response to acute stressors. Trends in Neurosciences, 304-314.

3 Lovallo, W.R., Robinson, J.L., Glahn, D.C. \& Fox, P.T. (2010). Acute effects of hydrocortisone on the human brain: an fMRI study. Psychoneuroendocrinology, 35, $15-20$.

4 van Marle, H.J., Hermans, E.J., Qin, S. \& Fernandez, G. (2009). From specificity to sensitivity: how acute stress affects amygdala processing of biologically salient stimuli. Biological Psychiatry, 66, 649-655.

5 Henckens, M.J.A.G., van Wingen, G.A. Joels, M. \& Fernandez, G. (2011). Timedependent corticosteroid modulation of prefrontal working memory processing. Proceedings of the National Academy of Sciences of the United States of America 108, 5801-5806.

6 Henckens, M.J.A.G. et al. (2012). Dynamically changing effects of corticosteroids on human hippocampal and prefrontal processing. Human Brain Mapping, 33, 2885-2897.

7 de Kloet, E.R., Joels, M. \& Holsboer, F. (2005). Stress and the brain: from adaptation to disease. Nature Reviews: Neuroscience, 6, 463-475.

8 Ulrich-Lai, Y.M. \& Herman, J.P. (2009) Neural regulation of endocrine and autonomic stress responses. Nature Reviews: Neuroscience, 10, 397-409.

9 van Marle, H.J., Hermans, E.J., Qin, S. \& Fernandez, G. (2010). Enhanced restingstate connectivity of amygdala in the immediate aftermath of acute psychological stress. Neuroimage, 53, 348-354.

10 Veer, I.M. et al. (2011). Beyond acute social stress: increased functional connectivity between amygdala and cortical midline structures. Neuroimage, 57, 1534-1541.

11 Clewett, D., Schoeke, A. \& Mather, M. (2013). Amygdala functional connectivity is reduced after the cold pressor task. Cognitive Affective \& Behavioural Neuroscience, 13, 501-518.

12 Vaisvaser, S. et al. (2013). Neural traces of stress: cortisol related sustained enhancement of amygdala-hippocampa functional connectivity. Frontiers in Human Neuroscience, 7.

13 Dedovic, K., D'Aguiar, C. \& Pruessner, J.C. (2009). What stress does to your brain: a review of neuroimaging studies. Canadian Journal of Psychiatry. Revue Canadienne de Psychiatrie, 54, 6-15.

14 Fox, M.D. \& Raichle, M.E. (2007) Spontaneous fluctuations in brain activity observed with functiona magnetic resonance imaging. Nature Reviews: Neuroscience, 8, 700-711.

15 McEwen, B.S. (2007). Physiology and neurobiology of stress and adaptation: central role of the brain. Physiological Reviews, 87, 873-904.

16 Phillips, M. L., Drevets, W. C., Rauch, S. L. \& Lane, R. (2003). Neurobiology of emotion perception I: The neural basis of norma emotion perception. Biological Psychiatry, 54, 504-514.

17 McGaugh, J.L. (2004). The amygdala modulates the consolidation of memories of emotionally arousing experiences. Annual Review of Neuroscience, 27, 1-28.

18 Roozendaal, B., McEwen, B.S. \& Chattarji, S. (2009). Stress, memory and the amygdala. Nature Reviews: Neuroscience, 10, 423-433.

19 Schwabe, L., Joels, M., Roozendaal, B Wolf, O.T. \& Oitzl, M.S. (2012). Stress 
effects on memory: an update and integration. Neuroscience and Biobehavioural Reviews, 36, 1740-1749.

20 Shin, L.M. et al. (2005). A functional magnetic resonance imaging study of amygdala and medial prefrontal cortex responses to overtly presented fearful faces in posttraumatic stress disorder Archives of General Psychiatry, 62, 273281.

21 Urry, H.L. et al. (2006). Amygdala and ventromedial prefrontal cortex are inversely coupled during regulation of negative affect and predict the diurna pattern of cortisol secretion among older adults. Journal of Neuroscience, 26, 4415 4425.

22 Banks, S.J., Eddy, K.T., Angstadt, M. Nathan, P.J. \& Phan, K.L. (2007) Amygdala-frontal connectivity during emotion regulation. Social Cognitive and Affective Neuroscience, 2, 303-312.

23 Gianaros, P.J. et al. (2008). Individual differences in stressor-evoked blood pressure reactivity vary with activation, volume, and functional connectivity of the amygdala. Journal of Neuroscience, 28 990-999.

24 Kern, S. et al. (2008). Glucose metabolic changes in the prefrontal cortex are associated with HPA axis response to a psychosocial stressor. Psychoneuroendocrinology, 33, 517-529.

25 Quaedflieg, C.W.E.M., Meyer, T. \& Smeets, T. (2013). The imaging Maastricht Acute Stress Test (iMAST): a neuroimaging compatible psychophysiological stressor Psychophysiology, 50, 758-766.

26 Pruessner, J.C. et al. (2008). Deactivation of the limbic system during acute psychosocial stress: evidence from positron emission tomography and functional magnetic resonance imaging studies. Biological Psychiatry, 63, 234-240.

27 Dedovic, K. et al. (2009). Neural correlates of processing stressful information: An event-related fMRI study. Brain Research 1293, 49-60.

28 Lang, P.J., Bradley, M.M. \& Cuthbert, B.N (2008). International Affective Picture System (IAPS): Affective Ratings of Pictures and Instruction Manual, Technical Report A-8.

29 Pruessner, J.C., Kirschbaum, C., Meinlschmid, G. \& Hellhammer, D.H. (2003). Two formulas for computation of the area under the curve represent measures of total hormone concentration versus time-dependen change. Psychoneuroendocrinology, 28, 916-931.

30 Miller, R., Plessow, F., Kirschbaum, C. \& Stalder, T. (2013). Classification Criteria for Distinguishing Cortisol Responders From Non-responders to Psychosocia Stress: Evaluation of Salivary Cortiso Pulse Detection in Panel Designs. Psychosomatic Medicine, 75, 832-840.

31 Weiskopf, N., Hutton, C., Josephs, O. \& Deichmann, R. (2006). Optimal EPI parameters for reduction of susceptibility-induced BOLD sensitivity losses: A whole-brain analysis at $3 \mathrm{~T}$ and 1.5 T. Neuroimage, 33, 493-504.

32 Goebel, R., Esposito, F. \& Formisano, E. (2006). Analysis of Functional Image Analysis Contest (FIAC) data with BrainVoyager QX: From single-subject to cortically aligned group general linear model analysis and self-organizing group independent component analysis. Human Brain Mapping, 27, 392-401.

33 Fox, M.D. et al. (2005). The human brain is intrinsically organized into dynamic, anticorrelated functional networks. Proceedings of the National Academy of Sciences of the United States of America, 102, 9673-9678.

34 Birn, R.M., Murphy, K., Handwerker, D. A. \& Bandettini, P.A. (2009). fMRI in the presence of task-correlated breathing variations. Neuroimage, 47, 1092-1104.
35 Scholvinck, M.L., Maier, A., Ye, F.Q., Duyn, J.H. \& Leopold, D.A. (2010). Neural basis of global resting-state fMRI activity. Proceedings of the National Academy of Sciences of the United States of America 107, 10238-10243.

36 Murphy, K., Birn, R.M., Handwerker, D. A Jones, T. B. \& Bandettini, P.A. (2009). The impact of global signal regression on resting state correlations: are anticorrelated networks introduced? Neuroimage, 44, 893-905.

37 Carbonell, F., Bellec, P. \& Shmuel, A. (2014). Quantification of the impact of a confounding variable on functional connectivity confirms anti-correlated networks in the resting-state. Neuroimage, 86, 343-353.

38 Shmueli, K. et al. (2007). Low-frequency fluctuations in the cardiac rate as a source of variance in the resting-state $\mathrm{FMRI} B O L D$ signal. Neuroimage, 38, 306-320.

39 Chang, C. et al. (2013). Association between heart rate variability and fluctuations in resting-state functional connectivity. Neuroimage, 68, 93-104.

40 Roy, A.K. et al. (2009). Functional connectivity of the human amygdala using resting state fMRI. Neuroimage, 45 , 614-626.

41 Robinson, J.L., Laird, A.R., Glahn, D.C., Lovallo, W.R. \& Fox, P.T. (2010) Metaanalytic connectivity modeling: delineating the functional connectivity of the human amygdala. Human Brain Mapping, 31, 173-184.

42 Stein, J.L. et al. (2007). A validated network of effective amygdala connectivity. Neuroimage, 36, 736-745.

43 Phillips, M., Ladouceur, C. \& Drevets, W. (2008). A neural model of voluntary and automatic emotion regulation: implications for understanding the pathophysiology and neurodevelopment of bipolar disorder. Molecular Psychiatry, 13, 833-857.
44 Li, H.J. et al. (2014). Examining brain structures associated with perceived stress in a large sample of young adults via voxel-based morphometry. Neuroimage, 92, 1-7.

45 Strelzyk, F. et al. (2012). Tune it down to live it up? Rapid, nongenomic effects of cortisol on the human brain. Journal of Neuroscience, 32, 616-625.

46 Henckens, M.J.A.G., Hermans, E.J., Pu, Z.W., Joels, M. \& Fernandez, G.N. (2009). Stressed Memories: How Acute Stress Affects Memory Formation in Humans. Journal of Neuroscience, 29, 10111-10119.

47 Sabatinelli, D., Bradley, M.M. Fitzsimmons, J.R. \& Lang, P.J. (2005). Parallel amygdala and inferotemporal activation reflect emotional intensity and fear relevance. Neuroimage, 24, 12651270.

48 Weissman, D.H., Roberts, K.C., Visscher, K.M. \& Woldorff, M.G. (2006). The neural bases of momentary lapses in attention. Nature Neuroscience, 9, 971-978.

49 Henckens M.J.A.G., van Wingen G.A., Joëls M, Fernández G. (2010). Time-dependent effects of corticosteroids on human amygdala processing. Journal of Neuroscience., 38, 12725-12732.

50 Kudielka, B.M., Hellhammer, D.H. \& Wust S. (2009). Why do we respond so differently? Reviewing determinants of human salivary cortisol responses to challenge. Psychoneuroendocrinology, 34, 2-18.

51 Joels, M. \& Baram, T.Z. (2009). The neurosymphony of stress. Nature Reviews Neuroscience, 10, 459-U484.

52 Sullivan, R.M. \& Gratton, A. (2002). Prefrontal cortical regulation of hypothalamic-pituitary-adrenal function in the rat and implications for psychopathology: side matters. Psychoneuroendocrinology, 27, 99-114.

53 Dedovic, K., Duchesne, A., Andrews, J., Engert, V. \& Pruessner, J.C. (2009). The 
brain and the stress axis: the neura correlates of cortisol regulation in response to stress. Neuroimage, 47, 864 871

54 Etkin, A., Egner, T., Peraza, D.M., Kandel E.R. \& Hirsch, J. (2006). Resolving emotional conflict: A role for the rostral anterior cingulate cortex in modulating activity in the amygdala. Neuron, 52, 1121-1121.

55 Etkin, A., Egner, T. \& Kalisch, R. (2011). Emotional processing in anterio cingulate and medial prefrontal cortex. Trends in Cognitive Sciences, 15, 85-93.

56 Etkin, A., Prater, K.E., Hoeft, F., Menon, V. \& Schatzberg, A.F. (2010). Failure of Anterior Cingulate Activation and Connectivity with the Amygdala During Implicit Regulation of Emotional Processing in Generalized Anxiety Disorder. American Journal of Psychiatry, 167, 545-554.

57 Johnstone, T., van Reekum, C.M., Urry H.L., Kalin, N.H. \& Davidson, R.J. (2007) Failure to regulate: Counterproductive recruitment of top-down prefrontalsubcortical circuitry in major depression. Journal of Neuroscience, 27, 8877-8884.

58 Bush, G., Luu, P. \& Posner, MI. (2000) Cognitive and emotional influences in anterior cingulate cortex. Trends in Cognitive Sciences, 4, 215-222.

59 McRae, K., Reiman, E.M., Fort, C.L., Chen, K. \& Lane, R.D. (2008). Association between trait emotional awareness and dorsal anterior cingulate activity during emotion is arousal-dependent. Neuroimage, 41, 648-655.

60 Seeley, W.W. et al. (2007). Dissociable intrinsic connectivity networks for salience processing and executive control. Journal of Neuroscience, 27, 23492356.

61 Davidson, R.J. (2004). What does the prefrontal cortex "do" in affect: perspectives on frontal EEG asymmetry research. Biological Psychology, 67, 219233

62 Quaedflieg, C.W.E.M., Meyer, T., Smulders, F.T.Y. \& Smeets, T. (2015). The functiona role of individual-alpha based frontal asymmetry in stress responding. Biological Psychology, 104, 75-81.

63 LeDoux, J.E. (2000). Emotion circuits in the brain. Annual Review of Neuroscience, 23, 155-184.

64 Roozendaal, B. (2003). Systems mediating acute glucocorticoid effects on memory consolidation and retrieval. Progress in Neuro-Psychopharmacology Biological Psychiatry, 27, 1213-1223.

65 Davidson, R.J. (2000). Affective style, psychopathology, and resilience: Brain mechanisms and plasticity. American Psychologist, 55, 1196-1214.

66 Waites, A.B., Stanislavsky, A., Abbott, D.F. \& Jackson, G.D. (2005). Effect of prior cognitive state on resting state networks measured with functional connectivity. Human Brain Mapping, 24, 59-68.

67 Stevens, W.D., Buckner, R.L. \& Schacter D.L. (2010). Correlated Low-Frequency BOLD Fluctuations in the Resting Human Brain Are Modulated by Recent Experience in Category-Preferentia Visual Regions. Cerebral Cortex, 20, 1997 2006.

68 Fair, D.A. et al. (2007). A method for using blocked and event-related fMRI data to study "resting state" functional connectivity. Neuroimage, 35, 396-405.

69 van de Ven, V., Wingen, M., Kuypers, K.P.C., Ramaekers, J.G. \& Formisano, E. (2013). Escitalopram decreases crossregional functional connectivity within the default-mode network. PloS One, 8.

70 Smeets, T. et al. (2012). Introducing the Maastricht Acute Stress Test (MAST): A quick and non-invasive approach to elicit robust autonomic and glucocorticoid stress responses. Psychoneuroendocrinology, 37, 1998-2008.

\section{Appendix: Supplemental Information}

\section{Neuroendocrine stress responses}

The timing of saliva sampling was based on previous work showing that cortisol typically peaks 20 to 40 -min after stress onset'. Practically, for each sample collection, participants were returned to the home position of the scanner, and a research assistant placed the Salivette in the mouth of the participant using sterile plastic tweezers. To facilitate sample collection and to minimize movement, this procedure was trained extensively beforehand during a simulation scan in a dummy MRI scanner.

Saliva samples were stored at $-20^{\circ} \mathrm{C}$ immediately upon collection. Cortisol levels were determined from the saliva samples using a commercially available kinetic reaction assay (Salimetrics, Penn State, PA) and luminescence immune assay kit (IBL, Hamburg, Germany), respectively. Mean intra- and inter-assay coefficients of variation are typically less than $5 \%$ for the cortisol analyses.

Table S1 Means ( \pm SEM) of subjective stress and cortisol (untransformed values). Grey areas indicate values used for calculation of the area under the curve (AUCi).

\begin{tabular}{|c|c|c|c|}
\hline Subjective stress $(0-100)$ & 74.2 & $(3.24)$ & \\
\hline Cortisol (nmol/l) & & & CORT-AUCi \\
\hline$t_{\mathrm{MRI}}$ & 9.80 & $(1.61)$ & \\
\hline$t_{\text {pre-stress }}$ & 8.10 & $(0.91)$ & \\
\hline$t_{+0}$ & 9.40 & $(1.12)$ & 108.49 \\
\hline$t_{+10}$ & $\mid 11.7$ & $(1.53)$ & (25.63) \\
\hline$t_{+30}$ & 10.4 & $(1.25)$ & \\
\hline$t_{+40}$ & 8.80 & $(0.84)$ & \\
\hline
\end{tabular}


Run 1 vs Run 2

The 84 pictures were presented for 1800-ms and the indoor/outdoor decision was made within $1800-m s$ after picture presentation. Pictures were interspersed with a variable interstimulus interval (ISI) $(2,4,6$, or 8 -s) during which a fixation cross was presented. The presentation order of the pictures was semi-randomized so that no more than three pictures of one condition appeared consecutively in order to avoid mood state induction ${ }^{2}$.
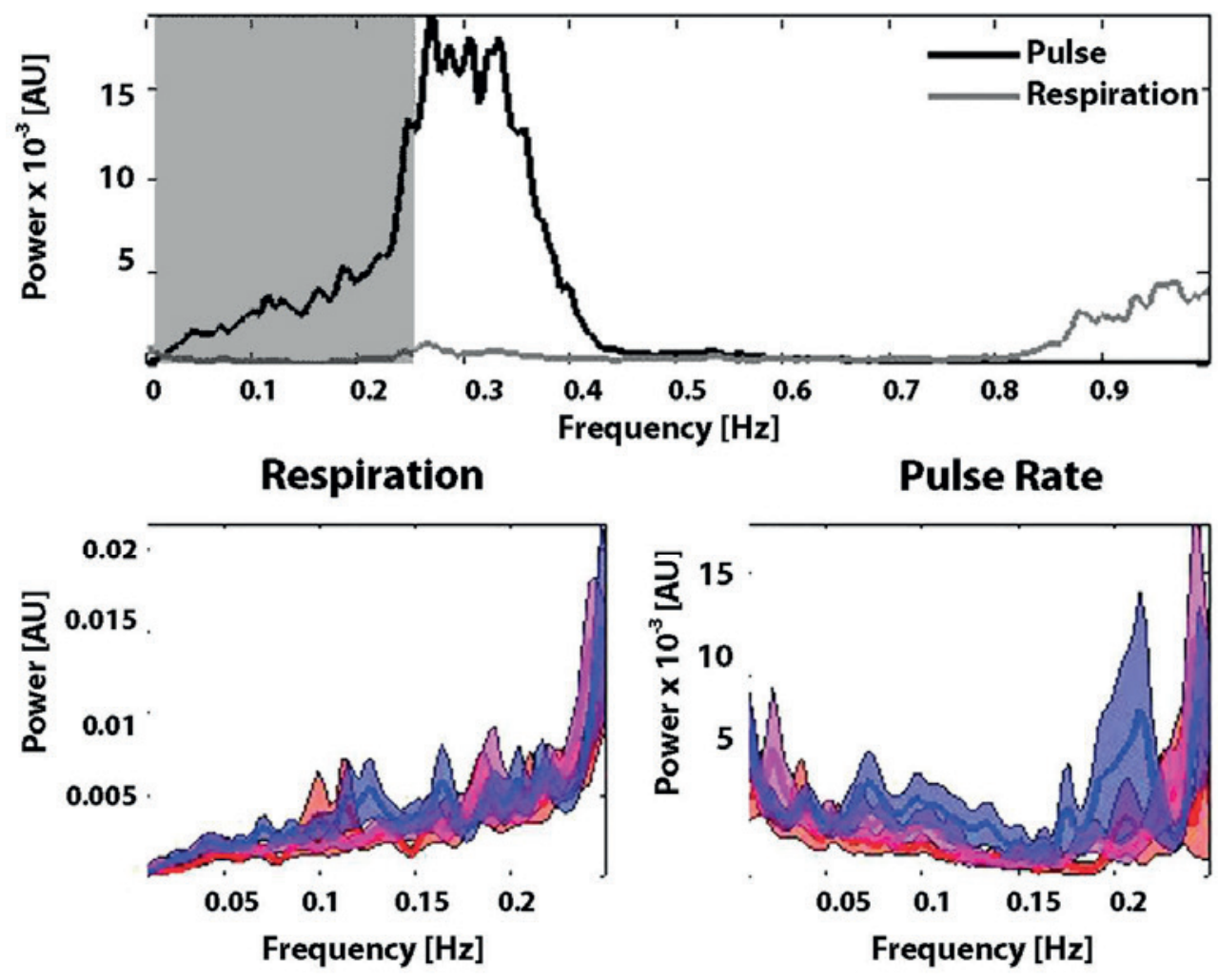

Rest,

Rest $_{2}$

Rest $_{3}$

Figure S1 Power spectral densities (PSDs) of the physiological responses. Upper panel: Mean PSDs of the pulse rate (black line) and respiration (grey line) across all participants and conditions. For visualization the frequency range is truncated to $1 \mathrm{~Hz}$. Grey area represents the frequency window coinciding with the scanner resolution of TR $=2 \mathrm{~s}$ (i.e., $0-0.25 \mathrm{~Hz}$ ). Lower panels: Mean (+- SEM area) PSDs of pulse rate (left) and respiration (right) for the scanner-relevant frequency range of the three resting-state measurements. ANOVAs across the plotted frequencies were not significant (all corrected $p s>0.05$ ).

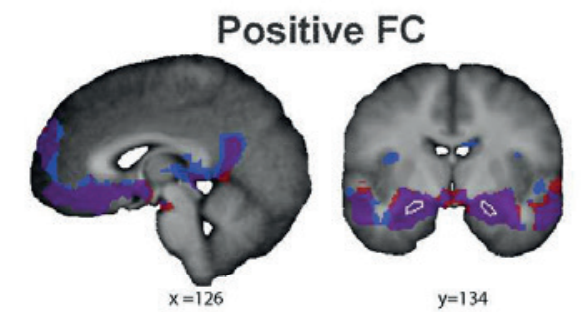

Negative FC

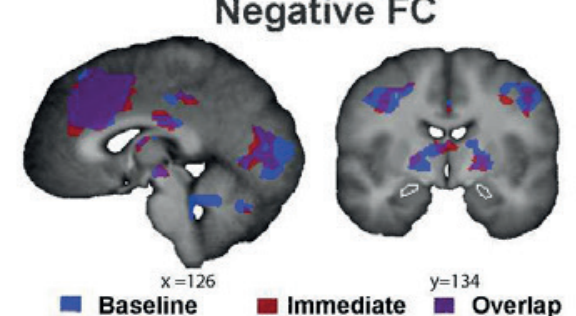

Baseline Immediate $\quad$ Oliserlap

Figure S2 Overlap between amygdala resting state functional connectivity maps. The overlap with the baseline measurement (i.e., run 1) is shown in purple in the pairwise maps. The amygdala seed used for the analysis is drawn in white. Statistical maps (FDR correction threshold of $q=.005$ ) are overlaid on the anatomical average of the participants. In the coronal view, the left side of the brain corresponds to the right hemisphere and vice versa.

\section{Supporting References}

Dickerson, S.S. \& Kemeny, M.E. (2004).

Acute stressors and cortisol

responses: A theoretical integration and synthesis of laboratory research. Psychological Bulletin, 130, 355-391.

2 Ritchey, M., Dolcos, F. \& Cabeza, R. (2008). Role of Amygdala Connectivity in the Persistence of Emotional Memories Over Time: An EventRelated fMRI Investigation. Cerebral Cortex, 18, 2494-2504.

\section{Run 1 vs Run 3}

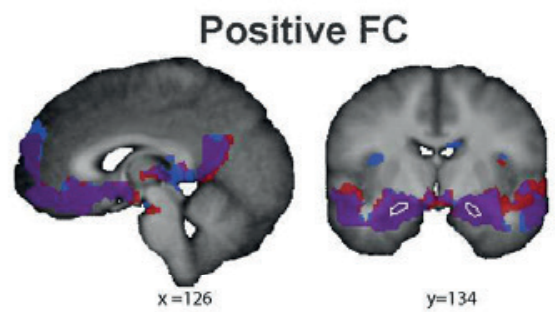

Negative FC

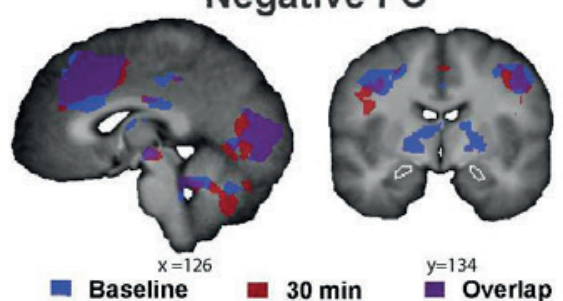




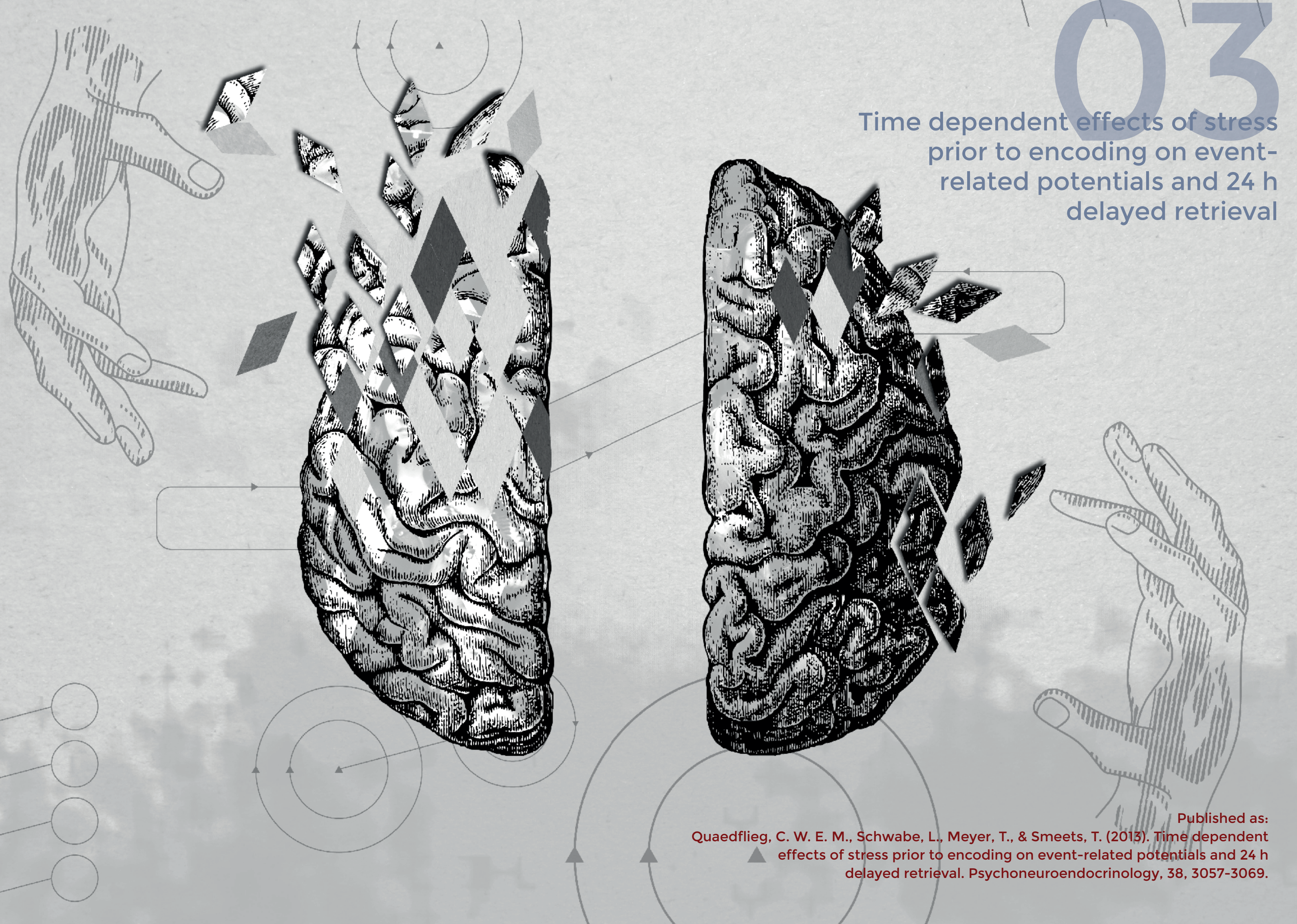




\section{Abstract}

Stress can exert profound effects on memory encoding. Here, we investigated whether (sub)cortical information processing during encoding and memory retrieval at a $24-h$ delayed test are affected by the temporal proximity between stress and memory encoding. Sixty-four participants engaged in the Maastricht Acute Stress Test (MAST) or a no-stress control condition either immediately before (i.e., proximate condition) or 30-min before (i.e., distant condition) a picture encoding task. In general, stress decreased the number of freely recalled and recognized pictures and increased the number of false alarms. However, timing of stress exposure did not differentially affect picture recall, recognition or selective attention processes (i.e., LPP). Nevertheless, stress-induced cortisol responses and correctly recognized neutral pictures were positively associated within the proximate stress condition but negatively associated within the distant stress condition. These findings suggest that the time at which a stressor is applied might differentially impact the association between stress-induced cortisol elevations and memory formation and indicate the need for a finer delineation of the time window during which glucocorticoids affect memory formation processes.

Keywords: late positive potentials (LPP), glucocortiocoids (GCs), Maastricht Acute Stress Test (MAST), emotional memory, event-related potential (ERP) 
When confronted with a stressful situation, our brain activates two physiological systems that allow us to cope effectively with the stressor. The rapidly acting autonomic nervous system (ANS) results in adrenaline and noradrenaline release and increased arousal, alertness, and focused attention. A second, slower mechanism involves the activation of the hypothalamic-pituitary-adrenal (HPA) axis that results in the secretion of the primary human glucocorticoid (GC) cortisol, which can bind to mineralocorticoid or glucocorticoid receptors (MRs and GRs, respectively)' ${ }^{1}$. The binding of cortisol to MRs and GRs in limbic structures, such as for example the hippocampus and amygdala, activates mechanisms involved in hippocampal plasticity and thereby can modulate memory processes ${ }^{2-4}$. Moreover, an interaction of cortisol with the noradrenergic system in the basolateral nucleus of the amygdala (BLA) $)^{3,5}$, a structure essential for the formation of emotional memories ${ }^{6}$, is said to be crucial for stress to affect emotional memory.

This stress-related release of cortisol and noradrenaline influences the quantity and quality of memory ${ }^{7}$. The effects of cortisol seems to be moderated by the memory phase that is targeted by stress and the emotional arousal elicited by the learned material ${ }^{8,9}$. In particular, stress can facilitate memory consolidation ${ }^{10,11}$, whereas stress before retention testing typically impairs memory retrieval ${ }^{12,13}$. Moreover, these effects are more pronounced for emotionally arousing material than for neutral stimuli5,10,13. It has also been suggested that during memory formation, the exact temporal dynamics of cortisol and noradrenaline release relative to the emotional memory encoding task are crucial for enhanced consolidation $^{14,15}$. Specifically, emotional experiences initiate a rapid enhancement of hippocampal neuroplasticity that is mediated by the amygdala and followed by stressrelated cortisol enhancement of hippocampal neuroplasticity via non-genomic membrane MR activity ${ }^{16,17}$. Over time, genomic GR actions induce a refractory state of the hippocampus, thereby impairing the processing of new information ${ }^{4,15,17,18}$. Support for this idea comes from a study by Zoladz and colleagues ${ }^{19}$, who found that stress applied immediately before encoding enhanced recognition of positive words while stress applied 30-min before encoding impaired recall of negative words $24-\mathrm{h}$ later.

One way to further test the time-dependent stress effect on memory formation is to use event related potentials (ERPs) obtained from electroencephalography (EEG). For instance, the time course of memory and emotion processes has been investigated with the late positive potential (LPP), an ERP over centro-parietal sites between 300 and $700 \mathrm{~ms}^{20,21}$.
The amplitude of the LPP appears to be larger for remembered stimuli (i.e., the 'subsequent memory effect'), especially when it concerns emotional material22-24. Weymar and colleagues ${ }^{25}$ recently used ERPs to investigate the effects of pre-learning stress on neutral and unpleasant picture recall $24-\mathrm{h}$ later. Although stress did not affect memory performance overall, the amplitude of the LPP during encoding of unpleasant pictures correlated positively with recall performance of unpleasant pictures 24 -h later in the stress, but not the control group. This suggests that pre-learning stress sensitizes the brain toward enhanced selective attention to unpleasant stimuli.

The current study draws upon the work of Weymar and colleagues ${ }^{25}$ and Zoladz and colleagues ${ }^{19}$ in that it investigated whether exposure to acute stress immediately (i.e., proximate stress group) or 30-min (i.e., distant stress group) before learning differentially affects emotional memory processing. This effect was assessed at the electrophysiological level by means of the LPP generated during memory encoding, and behaviourally using a delayed memory test that was carried out 24-h later. Given the known involvement of cortisol in the memory enhancing effects of stress on memory consolidation ${ }^{11}$, we also sampled salivary cortisol throughout the encoding session and related the stress-induced cortisol elevation to LPPs and 24-h delayed memory performance. Based on the theoretical framework of timing ${ }^{18}$, brain systems ${ }^{26}$, and previous work in humans ${ }^{19,25}$, we hypothesized that stress applied immediately before learning would improve 24-h delayed recall and recognition of emotional stimuli through enhanced selective attention (i.e., larger LPPs) and elevated cortisol levels, relative to a no-stress control condition. The opposite pattern of findings was expected for stress applied 30-min before learning.

\section{Methods}

\section{Participants}

Sixty-four right-handed healthy undergraduate men (mean age $=21.25$ years, $S D=2.5$; range: 18-31 years) were recruited to participate in this study via advertisements at Maastricht University. Participants were excluded if they had a history of psychiatric, neurologic, cardiovascular or neuroendocrine diseases, were considered heavy smokers (i.e. more than 15 cigarettes/day), used medication known to affect the ANS or HPA-axis, were regular drug users, or had a body mass index (weight in $\mathrm{kg} /(\text { height in } \mathrm{m})^{2}$ ) outside the 1830 range. Test protocols were approved by the standing ethics committee of the Faculty of 
Psychology and Neuroscience, Maastricht University. All participants signed a written informed consent and were given a small reward (course credit or monetary) in return for their participation.

\section{Stress manipulation}

The Maastricht Acute Stress Test (MAST) ${ }^{27}$ is a concise and valid procedure to reliably elicit robust subjective, autonomic and glucocorticoid stress responses. It consists of a 5-min preparation phase in which the task is explained and a 10-min acute stress phase that includes several exposures to cold pressor stress and various mental arithmetic challenges along with social-evaluative pressure (i.e., negative feedback). Specifically, in five trials that varied in duration from 60 to 90 -s, participants immersed their hand into ice water $\left(2^{\circ} \mathrm{C}\right.$ plexiglas box with an electrical cooler and a circulation pump from JULABO Labor technik, Seelbach, Germany). In between the hand immersion trials, participants engaged in mental arithmetic challenges in which they had to count backwards as fast and accurately as possible in steps of 17 starting at 2043 for 45,60 , or 90 -s. Whenever they counted too slowly or made a mistake, they received negative feedback (i.e., to count faster or start over again at 2043). To increase unpredictability and uncontrollability, participants were told that the order and duration of the hand immersion and mental arithmetic trials would be randomly chosen by the computer and that they would be videotaped for later analyses of their facial expressions, a procedure for which they had to provide written consent.

In the no-stress control condition, participants immersed their hand in lukewarm water $\left(35-37^{\circ} \mathrm{C}\right)$ and in between the hand immersion trials, performed a simple arithmetic task in which they had to count consecutively from 1 to 25 at their own pace and had to start anew at 1 when having reached 25. No feedback was given and participants were not videotaped. The duration and order of hand immersion and arithmetic trials paralleled that of the MAST.

\section{Subjective and neuroendocrine stress responses}

Subjective stress prior to and immediately following the MAST was assessed using the Negative Affect subscale of the Positive and Negative Affect Schedule, state version $(\mathrm{PANAS})^{28}$. The PANAS consists of two subscales that quantify current positive affect (PA) and negative affect (NA) using 5-point scales (anchors: $1=$ very slightly or not at all; $5=$ extremely). Higher scores on the NA scale are indicative of higher levels of experienced negative affect.

Neuroendocrine stress measures prior to and in response to the MAST were obtained with synthetic Salivette (Sarstedt, Etten-Leur, The Netherlands) devices 5 -min before $\left(t_{\text {pre- }}\right.$ stress) and four times after the MAST $\left(t_{+0}, t_{+20}, t_{+40}, t_{+50 \text { min }}\right.$ with reference to the end of the stressor). Samples were stored at $20^{\circ} \mathrm{C}$ until cortisol levels were determined by a commercially available luminescence immune assay kit (IBL, Hamburg, Germany). Mean intra- and inter-assay coefficients of variation are typically less than $5 \%$, and the lower and upper detection limits were $0.015 \mathrm{mg} / \mathrm{dl}(0.41 \mathrm{nmol} / \mathrm{l})$ and $4.0 \mathrm{mg} / \mathrm{dl}(110.4 \mathrm{nmol} / \mathrm{l})$, respectively.

\section{Memory task}

During the encoding phase, participants were presented with 36 negative and 36 neutral pictures from the International Affective Picture System (IAPS; see Appendix) ${ }^{29}$ on a monitor $(48 \mathrm{~cm} \times 27 \mathrm{~cm})$. The pictures belonged to one of the following five categories: person/action (e.g., neutral: woman answering phone; negative: carjacking), objects (e.g., neutral: clock negative: collection skulls), scenes (e.g., neutral: sunset at beach, negative: tornado), animals (e.g., neutral: butterfly on flower; negative: dog growling) or objects/ action (e.g., neutral: chess game; negative: car explosion). Pictures were presented for $3-s$ in full size (38 cm x 27 $\mathrm{cm}$ ) on black background, followed by a $50 \%$ size presentation of that image during which individual valence and arousal ratings were obtained by means of the Self-Assessment Manikin $(\mathrm{SAM})^{30}$ procedure to ensure deep picture encoding. The $50 \%$ picture was presented until the participant responded. An inter-stimulus interval of 2-s, during which a fixation cross was presented, was applied between all stimuli. IAPS pictures were chosen according to the IAPS valence and arousal ratings (neutral pictures: valence $M=5.84$, arousal $M=3.88$; negative pictures: valence $M=3.23$, arousal $M=5.50$ ). The ratings of the pictures obtained in the current sample were similar to the normative IAPS ratings (neutral pictures: valence $M=6.09$, arousal $M=4.06$; negative pictures: valence $M=2.90$, arousal $M=5.73$ ).

The recognition picture set presented 24-h later consisted of 72 pictures, of which $50 \%$ were old pictures, $25 \%$ were IAPS pictures not presented in the encoding task and were 
conceptually related to the old pictures (new similar/related neutral pictures) and $25 \%$ were new/different pictures (i.e., not previously presented and conceptually different). The new similar and new different pictures were matched to the old pictures on emotional valence and arousal (new similar/related neutral pictures: valence $M=6.01$, arousal $M=3.76$; negative pictures: valence $M=3.12$, arousal $M=5.71$; new/different neutral pictures: valence $M=5.69$, arousal $M=3.79$; negative pictures: valence $M=3.35$, arousal $M=5.45$ ) Performance on the recognition task was defined by hits (reflecting correct detection of old pictures), false alarms ( $F A$; reflecting incorrect detection of new pictures as being old), the discrimination index ( $P r$; reflecting correct discrimination of new from old pictures, i.e. (\# hits + $0.5 / \#$ old targets + 1) - (\# false alarms + 0.5/\# new targets + 1), and the bias index ( $B r$; \# false alarms $+0.5 / \#$ new targets +1$) /(1-P r)^{31}$. To avoid mood state induction, the presentation order of the pictures was semi-randomized so that no more than three pictures of one condition appeared consecutively in the picture encoding and the recognition part of the task.

\section{EEG data acquisition and analysis}

Electroencephalography (EEG) measurements were obtained during the encoding task, using 19 electrodes (F7, F3, Fz, F4, F8, C3, Cz, C4, CP1, CPz, CP2 P3, P1, Pz, P4, P2, O1, Oz, O2) attached to an elastic cap and a right mastoid according to the international 10-20 system. Two electrodes at the outer canthi of both eyes recorded horizontal eye movements and two electrodes above and below the left eye recorded vertical eye movements. The ground was located at AFz and the reference electrode was placed on left mastoid. A $500 \mathrm{~Hz}$ sampling rate and a band pass filter of $0.01-30 \mathrm{~Hz}$ was used to record EEG using Vision Recorder (Brain Products, Germany). Scalp-electrode impedance was kept below $5 \mathrm{kV}$ to ensure high-quality EEG recordings. Offline analyses were performed with Vision Analyzer 2.0 (Brain Products, Germany), including referencing to computer-linked mastoids reference, EOG correction using the Gratton and Coles algorithm, and baseline correction of 200-ms before stimulus onset. Epochs were made for each stimulus type separately from 200-ms before stimulus onset to 3000-ms after stimulus onset. Epochs containing EEG changes exceeding $75 \mathrm{mV}$ were automatically omitted from averages. Subjects were only included in the EEG analysis if they had at least 16 artefact free trials per condition (average number of trials was 27.3 for neutral and 27.2 for negative pictures). Data from two participants were excluded due to excessive artefacts and data of another participant were lost due to technical problems. The LPP was determined as the mean ERP amplitude in the window of 500-1000 ms over centro-parietal electrodes (CP1, CPz, CP2, P1, Pz, P2).

\section{Design and procedure}

A 2 (Condition: stress vs. control) $\times 2$ (Timing: proximate vs. distant) $\times 2$ (Picture Type: neutral vs. negative) mixed-model was employed, with Condition and Timing as between-subjects factors and Picture type as within subjects factor. Participants were randomly assigned to one of the four conditions and all testing took place between 12:30 and 18:00 $\mathrm{h}$ to control for the circadian rhythm of cortisol. Participants were asked to refrain from eating, exercising extensively or drinking anything but non-sparkling water for 2-h prior to both experimental sessions.

A timeline of the experimental sessions is displayed in Fig. 3.1. After their arrival in the laboratory, participants received information on the experimental procedure and gave written consent to participate in this study. Next, a saliva sample was taken with the explicit instruction that this sample would be immediately assayed to check whether they had adhered to our instructions not to eat, drink, etc. In reality, the sample was simply destroyed without being analysed. This was done to increase truth-telling behaviour when they were subsequently asked whether they had in fact adhered to the instructions. Next, participants were asked to drink $200 \mathrm{ml}$ of apple juice to standardize glucose levels ${ }^{32}$. Next, EEG equipment was prepared and an 8-min baseline EEG activity was measured. After assessing baseline Negative Affect (PANAS) and baseline cortisol ( $t_{\text {prestress }}$ ), the MAST or its control counterpart was performed. Either immediately (i.e., proximate condition) or 30-min (i.e., distant condition) following the MAST or no-stress control condition onset, participants performed the memory encoding task. Participants were told that we were interested in the individual differences in emotional reactivity elicited by the pictures and that the responses would be evaluated the next day. They were instructed to rate the pictures based on arousal and valence using the SAM. Filler questionnaires were used to bridge the time intervals. Participants in the distant groups filled the questionnaires out in between the stress induction and the encoding task while participants in the proximate group filled them out 
after the encoding task. After administration of the MAST or no-stress control condition, the PANAS and again 8-min baseline EEG activity were assessed. Participants were asked to provide additional saliva samples at four time points following the MAST or no-stress control condition: at $t_{+0}, t_{+20}, t_{+40}, t_{+50 m i n}$ with reference to the end of the stressor.

Twenty-four hours after the encoding session, participants returned to the lab. After a rest period of 10-min, a baseline saliva sample was obtained. Next, the participants had to complete the surprise free recall and recognition test. In the free recall test, participants were given 10-min to write down all pictures they could remember from the picture presentation procedure the day before. Participants were instructed to provide enough details so that two independent raters could identify and discriminate the picture from similar pictures. For the recognition test, participants were instructed to classify each picture as old or new. After all measures were completed, participants were debriefed and thanked for their participation.

\section{Distant stress / control ( 30 min before encoding)}
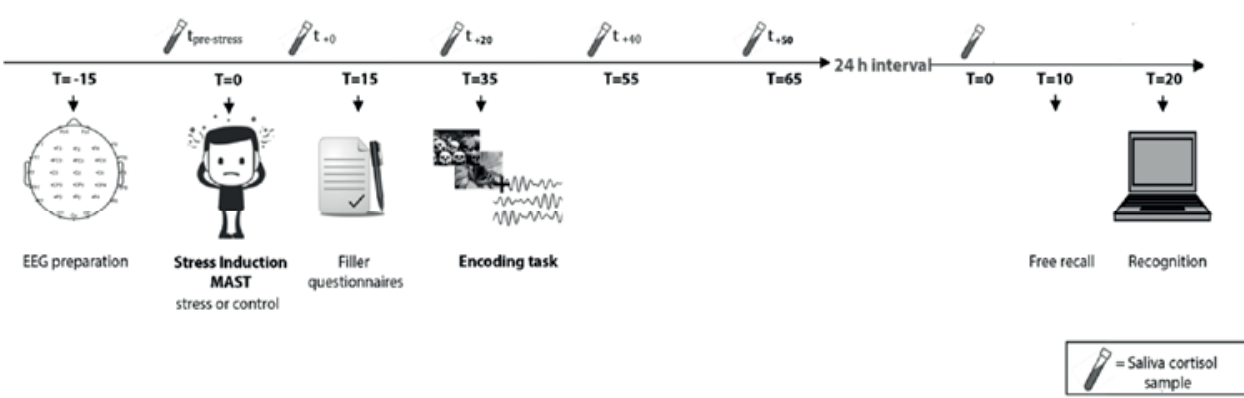

Proximate stress / control (immediately before encoding)

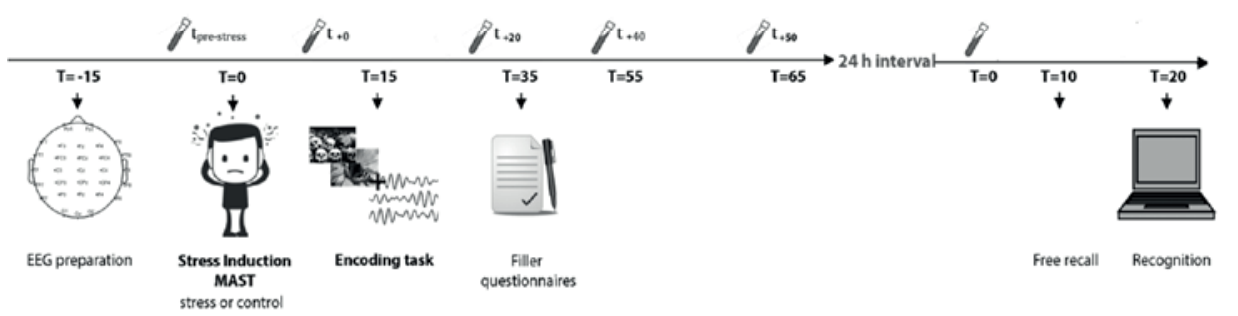

Figure 3.1 Overview of the study procedure. Either immediately (i.e., proximate condition) or 30 min (i.e., distant condition) following MAST or no-stress control condition onset, participants performed the memory encoding task. Abbreviations: MAST, Maastricht Acute Stress Test.

\section{Data analysis}

Subjective stress ratings were analysed using a 2 (Condition: stress vs. control) $\times 2$ (Timing: proximate vs. distant) $\times 2$ (Measurement: PANAS pre vs. PANAS post ) analysis of variance (ANOVA) with the latter as within subject factor. Cortisol data were log-transformed before analysis as Shapiro-Wilk tests of normality showed typical skewness of the data. Cortisol responses were analysed using a 2 (Condition: stress vs. control) $\times 2$ (Timing: proximate vs. distant) $\times 5$ (Measurement: tpre-stress vs. $t+0$ vs. $t+20$, vs. $t+40$ vs. $t+50 \mathrm{~min})$ ANOVA. For descriptive purposes, a responder rate was calculated representing participants with a cortisol increase equal to or larger than $2.5 \mathrm{nmol} / \mathbf{1}^{27,33}$, which is thought to reflect a cortisol secretory episode ${ }^{34}$. The percentage of participants who could be classified as cortisol responders (i.e., a cortisol increase $2.5 \mathrm{nmol} / \mathrm{l}$; see above) was $75 \%$ (12 out of 16) for the proximate stress group and $81 \%$ (13 out of 16) for the distant stress group. One participant out of 16 in the proximate control group showed a cortisol increase of $8.8 \mathrm{nmol} / \mathrm{l}$ and was excluded from further analyses. For each participant individually, the Area Under the Curve with respect to increase (AUCi) was calculated as a single measure of the total hormone (i.e., cortisol) concentration in response to the MAST or control task $\mathrm{k}^{35}$. The following formula was used to compute the AUCi: $\left(\left(\left(t_{+0}+t_{\text {pre-stress }}\right) / 2\right) \times 15\right)+\left(\left(\left(t_{+20}+t_{+0}\right) / 2\right) \times 20\right)+(((t+40+t+20 / 2)$ $x 20)+(((t+50+t+40) / 2) \times 15))+($ tpre-stress $\times(15+20+20+15))$. The effect of timing of stress exposure on memory performance (free recall \%, hits, $F A, P r$, and $B r$ ) and the LPP during encoding taking into account the different stimulus types was assessed using 2 (Picture Type: neutral, negative) x 2 (Condition: stress vs. control) $\times 2$ (Timing: proximate vs. distant) ANOVA with Picture Type as within-subject factor. To explore possible relationships between participants' physiological stress response and memory, bivariate Pearsons's correlations were conducted. We used a hypothesis driven approach for the correlations to limit the inflation of Type I error rates in these analyses. We used Greenhouse-Geisser correction when the assumption of sphericity in the repeated measures ANOVA was not met. All post hoc comparisons were performed using Bonferroni correction for multiple comparisons. In case of significant results, ANOVAs were supplemented with Partial Eta Squared $\left(\eta^{2}\right)$ values as a measure of effect size $\left(\eta^{2}{ }_{p}\right.$ of 0.01 indicate small effects, $\eta^{2}{ }_{p}$ of 0.06 medium effects, and $\eta^{2}{ }_{p}$ of 0.14 large effects) ${ }^{36}$ 


\section{Results}

\section{Subjective and neuroendocrine stress responses}

For PANAS Negative Affect, a significant Measurement x Stress interaction $\left(F_{(1,59)}=20.38, p<\right.$ $\left..001, \eta_{p}^{2}=.26\right)$ was found. Follow-up tests indicated that both stress groups showed an increase in negative affect in response to the stressor. Mean PANAS change after stress minus before stress ( $M_{\text {Post-Pre }}$ ) were $5.5(S E M=1.79)$ in the proximate stress group $\left(t_{(15)}=3.08, p\right.$ $=.008)$, and $2.7($ SEM $=0.90)$ in the distant stress group $\left(t_{(15)}=2.98, p=.009\right)$. Neither of the control conditions showed an increase (proximate control: $t_{(15)}=-2.12, p=.051, M_{\text {Post-pre }}=-0.94$, $\mathrm{SEM}=0.44$, distant control: $t_{(14)}=-1.07, p>.30, M_{\text {Post-Pre }}=-0.47$, SEM $\left.=0.43\right)$.

Mean salivary cortisol concentrations prior to and following the (no-stress control) MAST are shown in Fig. 3.2. For the salivary cortisol concentrations, a significant Measurement $x$ Stress interaction $\left(F_{(4,236)}=50.14, p<.001 ; \eta^{2}=.46 ; \varepsilon=.39\right)$ was found. Simple effects per saliva time point revealed higher cortisol concentrations in the stress groups compared with the control groups at $\mathrm{t}_{+0}\left(F_{(1,60)}=6.73, p=.012 ; \eta^{2}{ }_{\mathrm{p}}=.10\right), \mathrm{t}_{+20}\left(F_{(1,60)}=47.32, p\right.$ $\left.<.001 ; \eta_{\mathrm{p}}^{2}=.44\right), \mathrm{t}_{+40}\left(F_{(1,60)}=32.33, p<.001 ; \eta_{\mathrm{p}}^{2}=.35\right)$ and $\mathrm{t}_{+50 \mathrm{~min}}\left(F_{(1,60)}=23.47, p<.001 ; \eta^{2}{ }_{\mathrm{p}}\right.$ $=.28)$, but not at $\mathrm{t}_{\text {pre-stress }}\left(F_{(1,60)}=0.10 ; p=.76\right)$. Moreover, a univariate ANOVA indicated that the cortisol baseline during the recall task on day 2 did not differ between the four groups (Stress: $F_{(1,60)}=1.05, p=.31$; Measurement: $\left.F_{(1,60)}=0.04, p=.95\right)$. None of the other interaction and main effects was significant.

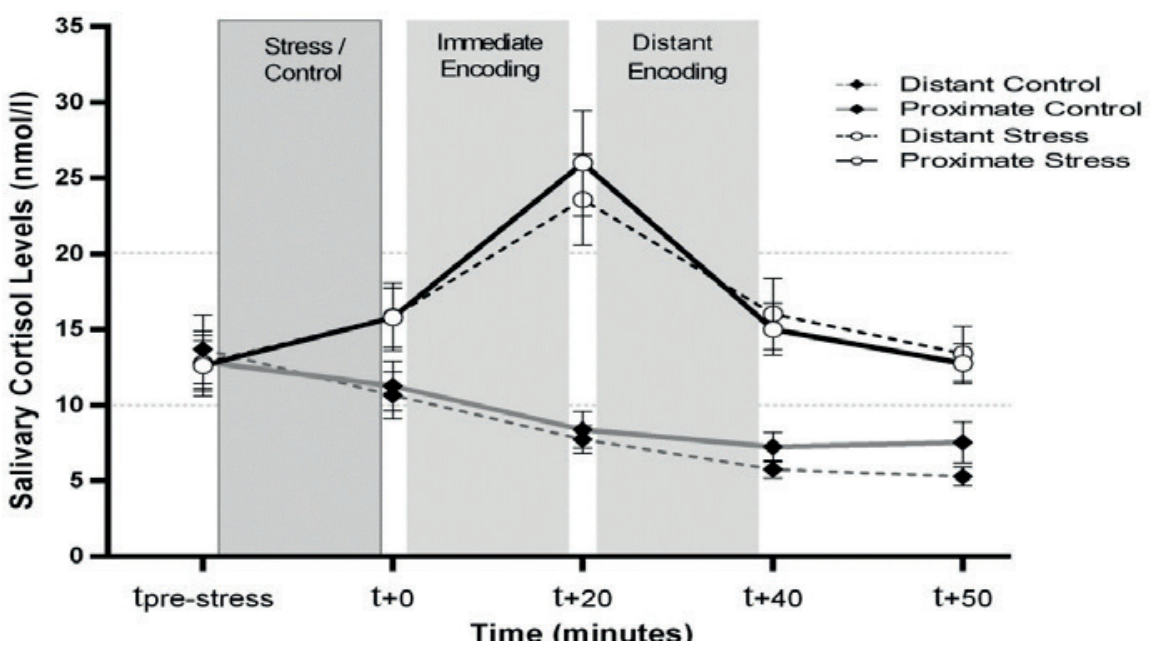

Figure 3.2 Salivary cortisol responses to the Maastricht Acute Stress Test (MAST). Graphs show means $\pm \mathrm{SE}$

\section{Behavioural results}

Mean scores derived from the free recall and recognition test are shown in Table 3.1. Free recall performance was affected by the emotional content of the pictures, as demonstrated by a significant main effect of Picture Type $\left(F_{(1,58)}=60.26, p<.001 ; \eta^{2}{ }_{p}=.51\right)$, with negative pictures being remembered better. Stress and Timing did not differentially affect the recall per picture type, as indicated by the non-significant Picture Type $\mathrm{x}$ Stress $\mathrm{x}$ Timing interaction $\left(F_{(1,58)}=0.26, p=.61\right)$ and the finding that none of the other interactions were significant. There was however a significant main effect of Stress $\left(F_{(1,58)}=6.88, p=.01 ; \eta^{2}{ }_{p}\right.$ $=.11$ ), with stress decreasing the number of pictures that were correctly remembered. Within the stress groups, Pearson's correlations between the percentage correct free recall of negative and neutral pictures, on the one hand, and the stress-induced changes in cortisol (AUCi), on the other hand, were performed to evaluate the relationship between participants' physiological stress responses and long-term memory performance. All correlations were non-significant ( $p^{\prime} s>.16$ ).

The analysis of the recognition data defined by the percentage hits (correct detection of old pictures) did not reveal any interaction or main effects involving Picture Type, Stress or Timing (all $p^{\prime} s>$.13). Yet, the analysis of false alarms revealed a main effect of Stress ( $F_{(1,}$ $\left.{ }_{58)}=7.35, p=.009 ; \eta_{\mathrm{p}}{ }_{\mathrm{p}}=.11\right)$, with stress increasing the number of false alarms, as well as a main effect of Picture Type $\left(F_{(1,58)}=20.45, p<.001 ; \eta^{2}{ }_{p}=.26\right)$, with negative pictures producing higher false alarm rates (no significant interactions emerged; all $p^{\prime} s>.15$ ). Moreover, the number of false alarms of neutral pictures was negatively related to the stressinduced changes in cortisol (AUCi) in the proximate condition $\left(r_{(15)}=-.53, p=.04\right)$.

The analysis of the discrimination index Pr revealed that recognition performance was affected by the emotional content of the pictures, as demonstrated by a significant main effect of Picture Type $\left(F_{(1,58)}=16.90, p<.001 ; \eta^{2}{ }_{p}=.22\right)$, with neutral pictures being better discriminated. Stress and Timing did not differentially affect recall per picture type, as indicated by the non-significant Picture Type $x$ Stress $x$ Timing interaction $\left(F_{(1,58)}=0.06, p\right.$ $=.80$ ), and none of the other interactions were significant. There was however a significant main effect of Stress $\left(F_{(1,58)}=4.24, p=.04 ; \eta^{2}=.07\right)$, with stress decreasing the discrimination 
of old/new pictures!. Moreover, the correct recognition of neutral pictures correlated with the stress-induced changes in cortisol (AUCi). Specifically, in the Proximate stress group, a positive correlation $\left(r_{(15)}=.52, p=.05\right)$ between AUCi and the number of correct recognized neutral pictures was found. While in the distant stress group, a negative correlation $\left(r_{(16)}=-\right.$ $.50, p=.05)$ between $\mathrm{AUCi}$ and the number of correct recognized neutral pictures was found (see Fig. 3.3). These correlations with the cortisol response (AUCi) are significantly different $(Z=2.85, p<.001)$. Importantly, these effects were obtained without group differences in $B r$ (all $\left.p^{\prime} s>.14\right)^{\prime \prime}$.

Table 3.1 Means ( $\pm \mathrm{SE}$ ) of Long-term (24-h) memory for negative and neutral pictures displayed as percentage free recall, percentage hits, false alarms, corrected recognition $(P r)$, and bias index $(B r)$.

\begin{tabular}{|c|c|c|c|c|}
\hline & $\begin{array}{l}\text { Proximate } \\
\text { Stress }\end{array}$ & $\begin{array}{l}\text { Distant } \\
\text { Stress }\end{array}$ & $\begin{array}{l}\text { Proximate } \\
\text { Control }\end{array}$ & $\begin{array}{l}\text { Distant } \\
\text { Control }\end{array}$ \\
\hline Neutral & $10.19(1.07)$ & $11.81(1.35)$ & $13.37(1.48)$ & $14.26(1.86)$ \\
\hline Negative & $18.52(1.79)$ & $20.14(1.78)$ & $23.61(1.81)$ & $22.22(1.94)$ \\
\hline \multicolumn{5}{|c|}{ Recognition (Hits \%) } \\
\hline Neutral & $95.83 \quad(0.98)$ & $95.31(1.26)$ & $95.31(1.54)$ & $96.48(1.16)$ \\
\hline Negative & $94.97(1.30)$ & $94.44(1.43)$ & $94.44(1.39)$ & $95.37(1.29)$ \\
\hline \multicolumn{5}{|c|}{ False Alarms (FA \%) } \\
\hline Neutral & $9.72 \quad(2.85)$ & $9.72 \quad(2.98)$ & $4.86(1.89)$ & $6.30 \quad(2.48)$ \\
\hline Negative & $16.67(2.87)$ & $19.10(3.29)$ & $9.03(1.96)$ & $10.37(2.23)$ \\
\hline \multicolumn{5}{|l|}{ Recognition (Pr) } \\
\hline Neutral & $0.91 \quad(0.02)$ & $0.90 \quad(0.02)$ & $0.93 \quad(0.02)$ & $0.93 \quad(0.02)$ \\
\hline Negative & $0.87 \quad(0.02)$ & $0.85 \quad(0.03)$ & $0.90 \quad(0.02)$ & $0.90 \quad(0.02)$ \\
\hline \multicolumn{5}{|l|}{ Bias Index (Br) } \\
\hline Neutral & $0.49 \quad(0.06)$ & $0.50 \quad(0.06)$ & $0.40 \quad(0.05)$ & $0.48 \quad(0.06)$ \\
\hline Negative & $0.58 \quad(0.06)$ & $0.62 \quad(0.05)$ & $0.50 \quad(0.04)$ & $0.53 \quad(0.05)$ \\
\hline
\end{tabular}

' The recognition task consisted of $25 \%$ new similar and $25 \%$ new different pictures. The analysis of the recognition memory $(P r)$ separately for new similar and new different items yielded highly similar results as the analysis of the overall $P r$, with stress decreasing the number of recognized pictures, independent of the picture type or the timing of the stressor.

"In the current sample, seven non-responders (i.e. participants who did not show a cortisol elevation > $2.5 \mathrm{mmol} / \mathrm{l}$ to the MAST) were identified. When the analyses were restricted to data from only cortisol responders, the $2 \times 2 \times 2$ ANOVAs yielded highly similar results.

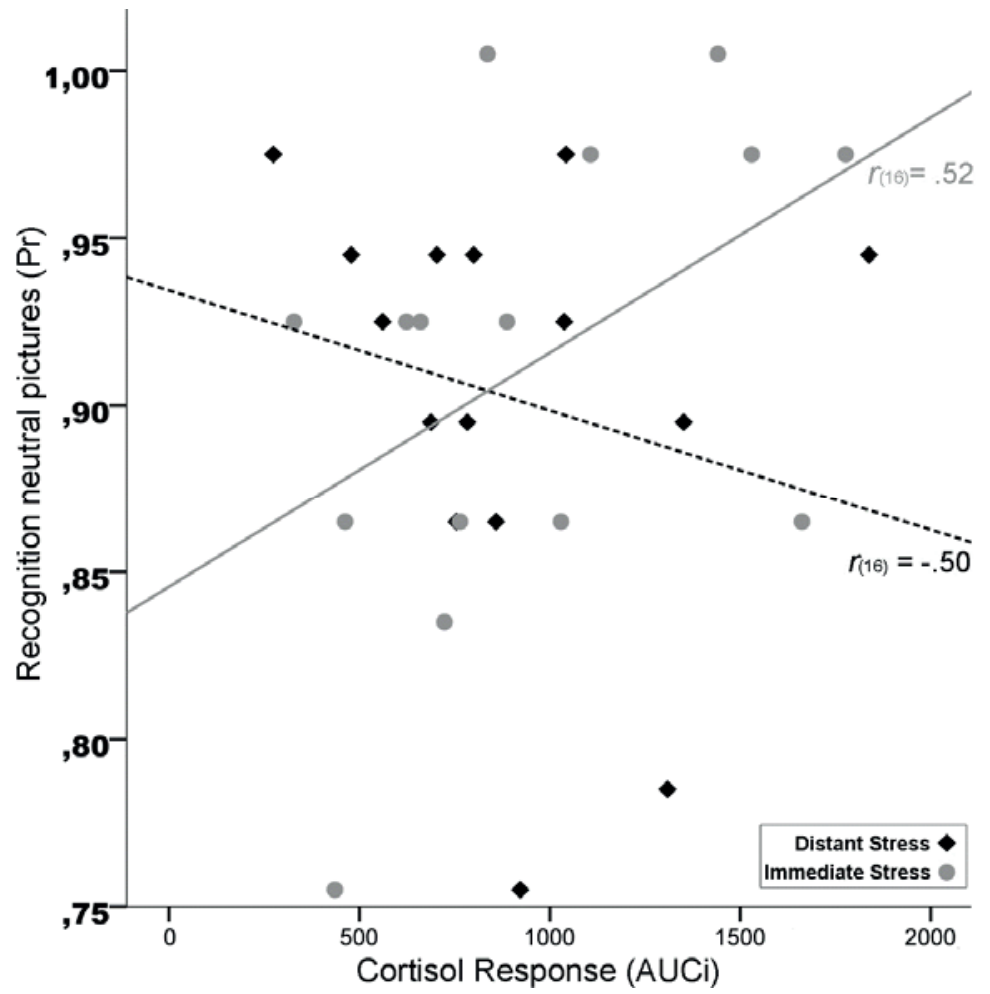

Figure 3.3. Correlations between the cortisol response (AUCi) and the recognition performance $\mathrm{Pr}$ ) of neutral pictures. Stress-induced cortisol changes are reversely associated with subsequen recognition of neutral pictures in the proximate and distant stress groups. Note that the two correlations significantly differ from each other $(p<.001)$.

\section{ERP results: late positive potential}

The overall shape of encoding ERPs was similar for neutral and negative pictures (see Fig. 3.4 and Fig. 3.5). As expected, mean LPP amplitudes for negative pictures were more positive going than for neutral pictures (Neutral $M=6.25, S E M=0.52$; Negative $M=8.59, S E M=0.68$; $\left.F_{(1,56)}=26.24, p<.001 ; \eta^{2}{ }_{p}=.32\right)$. Stress and Timing did not differentially affect the LPP in the 500-1000 ms window per picture type, as indicated by the non-significant interaction and main effects (Stress $x$ Timing $x$ Picture Type interaction $F_{(1,56)}=1.22, p=.28$ ). Within the stress groups, Pearson's correlations between the amplitude of the LPP in the 500-1000 ms window of negative and neutral pictures, on the one hand, and stress-induced changes in cortisol $(\mathrm{A} \cup \mathrm{C} \mathrm{i})$, on the other hand, were performed to evaluate the relationship between participants' physiological stress responses and information processing. In the proximate 
stress group, AUCi correlated positively with the amplitude of the LPP for negative pictures $\left(r_{(14)}=.54, p=.05\right)$, while in the distant stress group a negative correlation $\left(r_{(16)}=-.51, p=.05\right)$ between AUCi and the amplitude of the LLP for neutral pictures was found.

Based on visual inspection, the effect of Stress and Timing on the late LPP was also analysed. The late LPP was scored by mean activity in successive $500 \mathrm{~ms}$ windows from 1 to $2.5 \mathrm{~s}$. The ANOVAs of the LPP in the $1000-1500 \mathrm{~ms}, 1500-2000 \mathrm{~ms}$, and $2000-2500 \mathrm{~ms}$ window all indicated a main effect of Picture Type, with negative pictures eliciting a larger (more positive) LPP irrespective of Stress or Timing $\left(F_{(1,56)}=8.59, p<.005 ; \eta^{2}{ }_{\mathrm{p}}=.13 ; F_{(1,56)}=12.00, p\right.$ $=.001 ; \eta_{\mathrm{p}}^{2}=.18 ; F_{(1,56)}=3.03, p=.09 ; \eta_{\mathrm{p}}^{2}=.05$, respectively). Separate ANOVAs per picture type for the LPP in the 1500-2000 ms window revealed a main effect of Stress at trend level for neutral pictures $\left(F_{(1,56)}=3.42, p=.07 ; \eta_{\mathrm{p}}^{2}=.06\right)$ with stress decreasing (less positive) the LPP in the 1500-2000 ms time window. No interaction or main effects were significant for negative pictures or for the LPP in the 1000-1500 and 2000-2500 ms window"'.

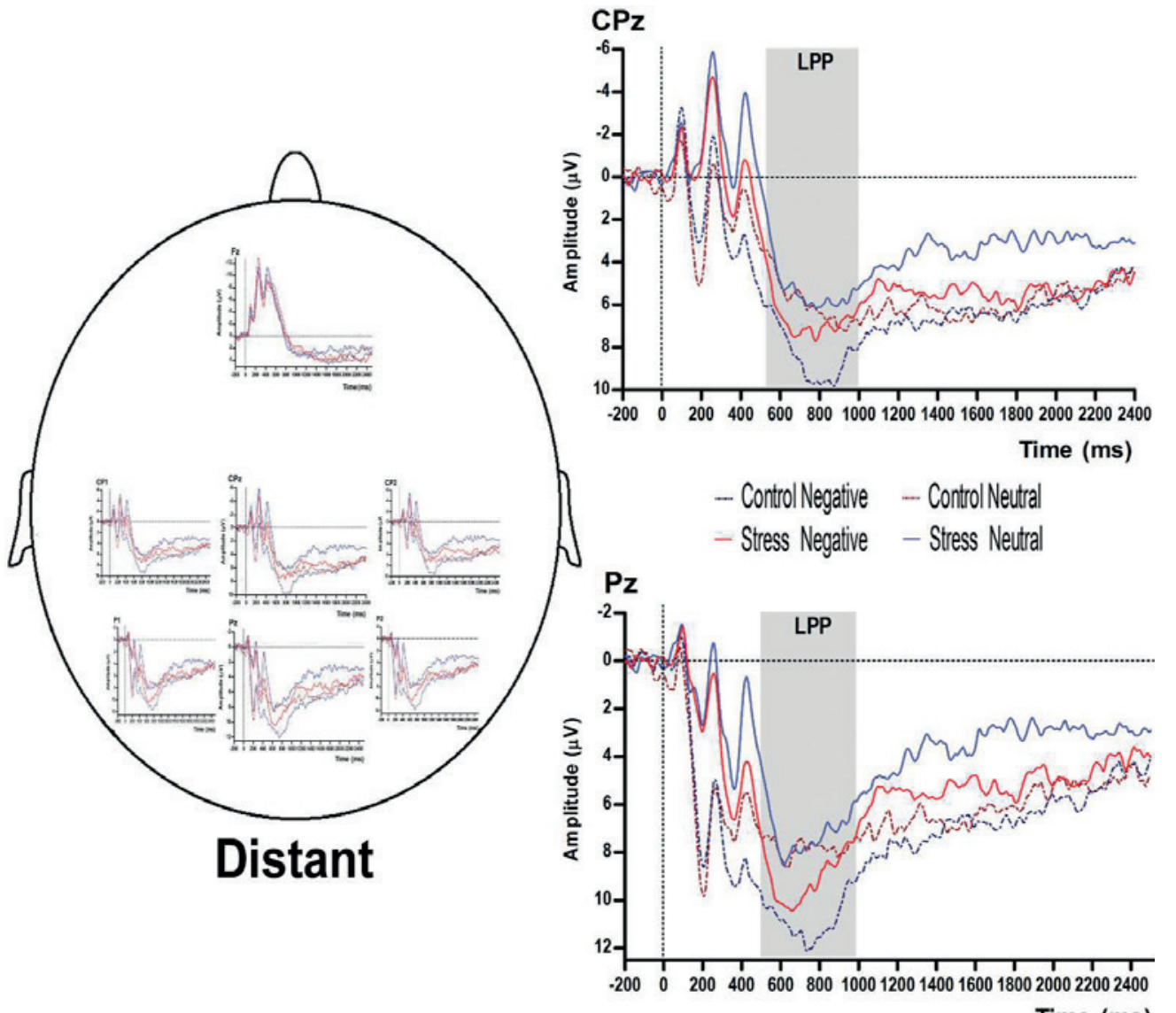

Time (ms)

Figure 3.4 Grand-averaged ERP waveforms over two scalp electrodes (CPz and Pz) for all neutral and negative pictures for the distant condition (control dotted line; stress solid line). The LPP was determined as the mean ERP amplitude in the window of 500-1000 ms, 1000-1500 ms, 1500-2000 ms and 2000-2500 ms over a cluster of centro-parietal electrodes (CP1, CPz, CP2, P1, Pz, P2). The LPP was larger for negative pictures compared to positive pictures $(p<.001)$. For the LPP in the 1500 $2000 \mathrm{~ms}$ window a main effect of stress was found with stress decreasing the LPP for neutral pictures $(p=.05)$. 


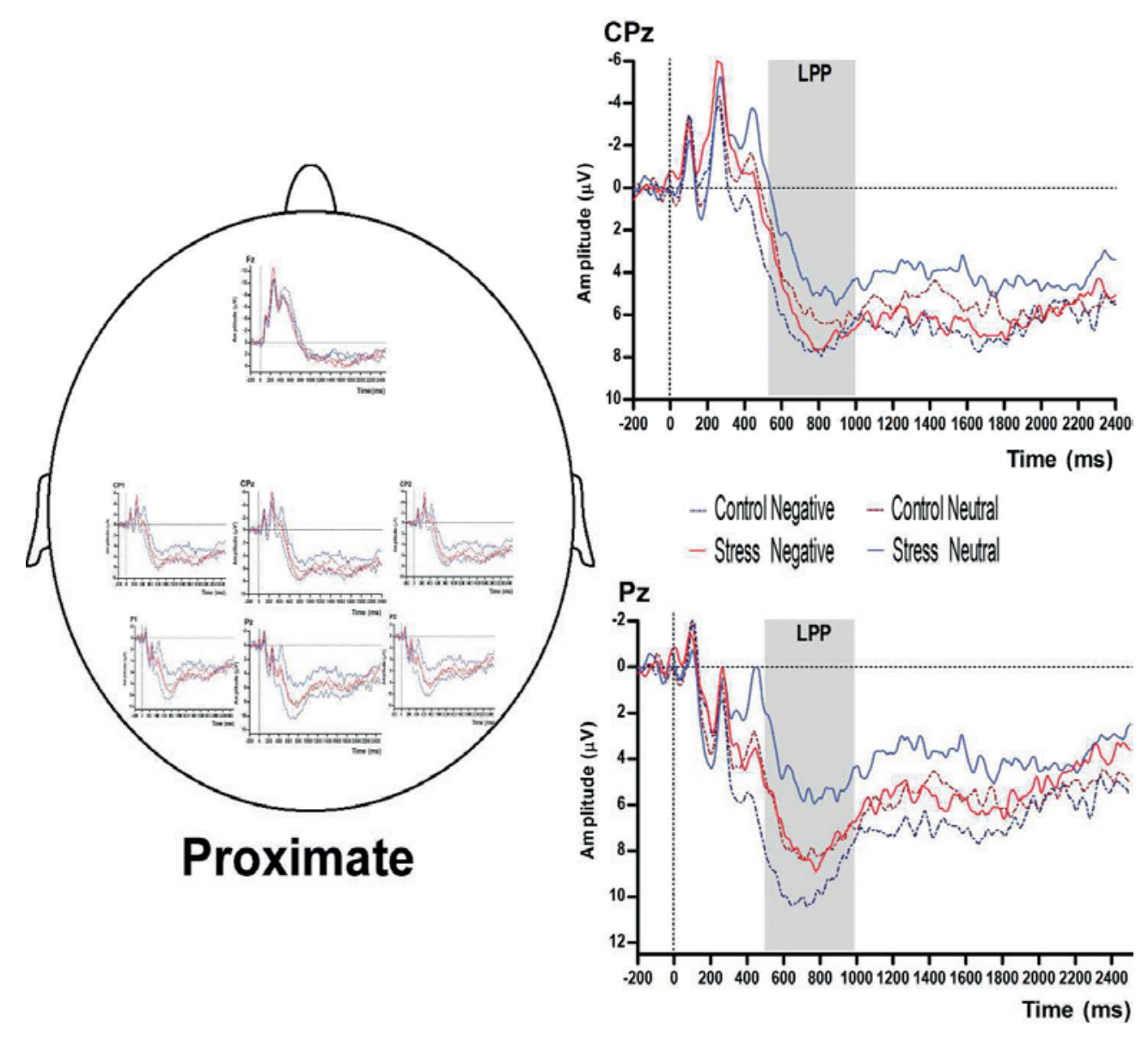

Figure 3.5 Grand-averaged ERP waveforms over two scalp electrodes ( $\mathrm{CPz}$ and Pz) for all neutral and negative pictures for the proximate condition (control dotted line; stress solid line). The LPP was determined as the mean ERP amplitude in the window of 500-1000 ms, 1000-1500 ms, 1500-2000 ms and 2000-2500 ms over a cluster of centro-parietal electrodes (CP1, CPz, CP2, P1, Pz, P2). The LPP was larger for negative pictures compared to positive pictures $(p<.001)$. For the LPP in the 1500 $2000 \mathrm{~ms}$ window a main effect of stress was found with stress decreasing the LPP for neutral pictures $(p=.05)$.

\section{Exploratory analysis of early ERPs}

A data driven approach was chosen to evaluate the effects of stress on early ERP components. Visual inspection served to determine areas of interest, which are shown in Fig. 3.6 \& 3.7. The $\mathrm{N} 100$ was measured as the mean amplitude in the window of 75-125 ms at Fz, the P200 in the window of $150-220 \mathrm{~ms}$ at Pz, and the N200 was in the window of 220$300 \mathrm{~ms}$ at $\mathrm{CPz}$.

For the N100, a significant Stress $x$ Timing $\times$ Picture Type interaction $\left(F_{(1,56)}=6.63, p=\right.$ $.01 ; \eta^{2}=.11$ ) was found. Follow-up analyses per Picture Type revealed no interaction or main effects for the negative ( $p^{\prime} s>.10$ ) or the neutral pictures ( $p^{\prime} s>.38$ ).

For the P200, a Stress $x$ Timing $x$ Picture Type interaction at trend level was found $\left(F_{(1,56)}=3.75, p=.06 ; \eta^{2}=.06\right)$. The corresponding two-way interactions for each picture type were significant (negative pictures: Stress $x$ Timing $F_{(1,56)}=5.70, p=.02 ; \eta_{p}^{2}=.09$; neutral pictures: Stress $x$ Timing $\left.F_{(1,56)}=13.32, p<.001 ; \eta^{2}{ }_{p}=.19\right)$. Post-hoc analyses per timing revealed that for both negative and neutral pictures, stress 30-min before encoding decreased (less positive) the P200 compared to the control group (negative pictures: $F_{(1,28)}=$ 9.83, $p<.005 ; \eta^{2}=.26$; neutral pictures: $\left.F_{(1,28)}=19.15, p<.001 ; \eta^{2}{ }_{p}=.41\right)$, whereas the effect of stress was not significant for the proximate condition in any of the separate ANOVAs per picture type $\left(p^{\prime} s>.40\right)$.

For the N200, a significant Stress $x$ Timing $x$ Picture Type interaction $\left(F_{(1,56)}=7.15, p=\right.$ $.01 ; \eta_{p}^{2}=.11$ ) was found. Follow-up analyses per Picture Type revealed for neutral pictures a Stress $x$ Timing interaction at trend level $\left(F_{(1,56)}=2.85, p=.10 ; \eta^{2}=.05\right)$, but no interaction or main effects for negative pictures (all $p$ 's $>.12$ ). For neutral pictures, simple effects per timing suggest that stress 30-min before encoding increased the N200 (more negative) compared to the control group $\left(F_{(1,28)}=6.29, p=.02 ; \eta^{2}=.18\right)$, whereas this stress effect remained nonsignificant in the proximate stress group. 


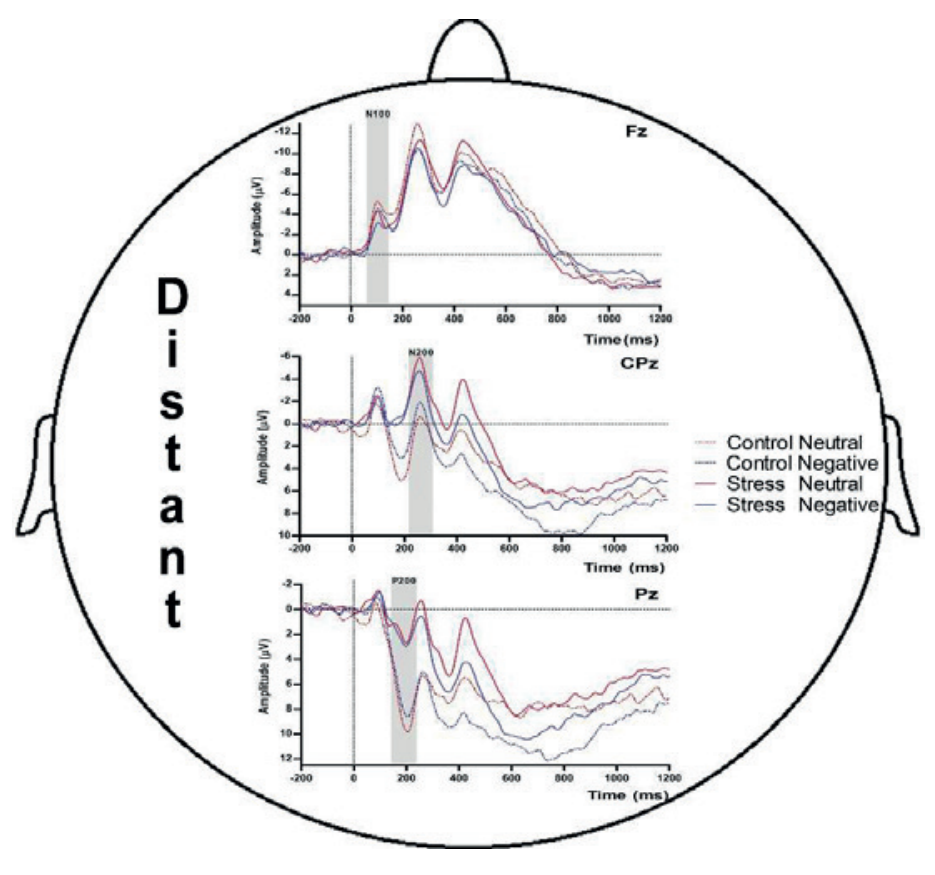

Figure 3.6 Grand-averaged waveforms depicting early ERP components for all neutral and negative pictures for the distant condition (control dotted lines; stress solid lines). Note that the P200 (150$220 \mathrm{~ms}$ ) for both pictures was decreased by stress 30-min before encoding (both $p^{\prime} \mathrm{s}<.01$ ). The N200 $(220-300 \mathrm{~ms})$ for neutral pictures was increased by stress 30-min before encoding $(p=.02)$.

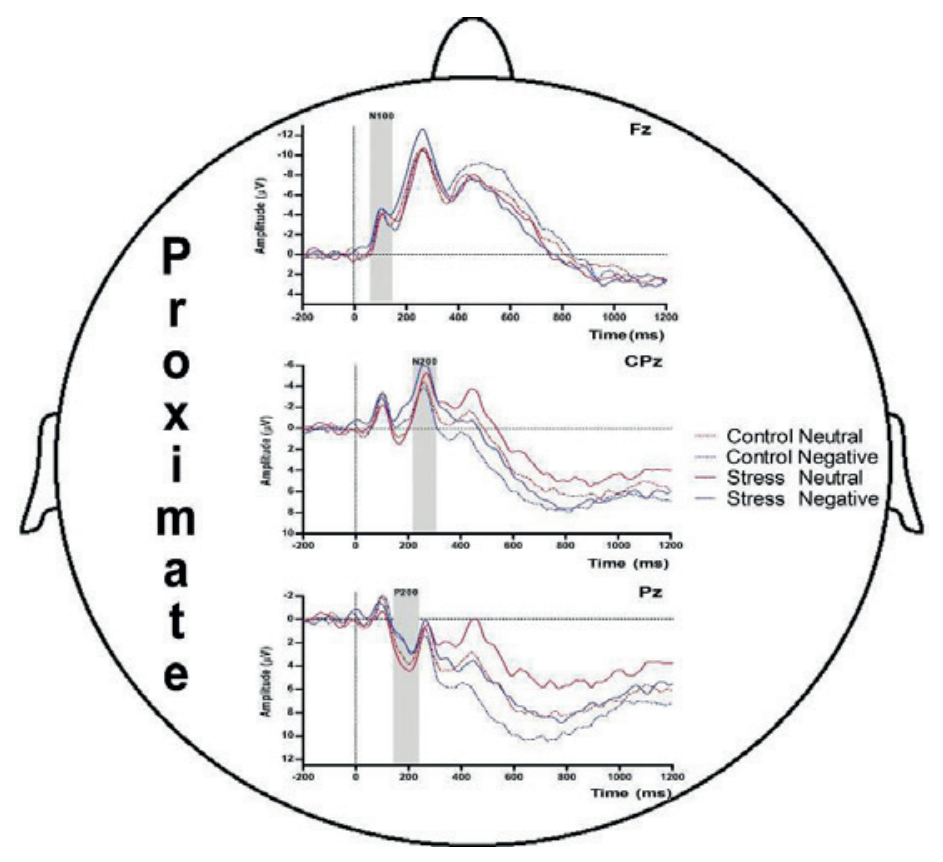

Figure 3.7 Grand-averaged waveforms depicting early ERP components for all neutral and negative pictures for the proximate condition (control dotted lines; stress solid lines).

\section{Discussion}

The primary aim of the current study was to investigate the influence of stress applied immediately or 30-min prior to encoding on information processing and on long-term (24h) memory performance. Results demonstrate that participants in both stress groups displayed significant stress responses in terms of subjective negative affect scores and cortisol levels compared with the no-stress control conditions. In general, stress decreased the number of freely recalled and recognized pictures, increased the number of false alarms, and decreased the LPP in the 1500-2000 ms window of neutral pictures. Moreover, stress-induced cortisol responses (AUCi) and correctly recognized neutral pictures were positively associated within the proximate stress group, whereas they were negatively associated within the distant stress group.

The present finding of stress increasing the number of false alarms is in line with previous research suggesting specific memory alternations due to stress-induced cortisol levels ${ }^{37}$. This increase in false alarms was not produced by an effect of stress on the general probability of answering yes as indicated by the non-significant effect of stress on the response bias. This latter finding to some extent disagrees with previous work showing a more liberal response bias for neutral information as a consequence of a stress-induced hyper vigilant neural state resulting in more generalized memory encoding ${ }^{38}$. Nevertheless, timing of the stressor did not differentially affect the number of false alarms. Moreover, in contrast to Zoladz and colleagues ${ }^{19}$, a differential effect of proximate versus distant stress on subsequent memory performance was not found, although we did find opposite correlations between the stress-induced cortisol response and the correct recognition of neutral pictures in the two stress timing groups. Zoladz and colleagues ${ }^{19}$ found an enhancing effect of stress applied immediately prior to learning for positive words on recognition memory and an impairing effect of stress applied 30-min prior to learning for negative words on free recall in cortisol responders. In the same vein, we found that stressinduced cortisol responses and correctly recognized neutral pictures were positively associated within the proximate stress group, but negatively within the distant stress group. Another apparent difference with Zoladz and colleagues ${ }^{19}$ is that we found associations with cortisol responses only for correct recognition of neutral pictures but not for the emotional pictures. Methodological differences can at least in part explain these divergent findings. For example, while Zoladz and colleagues ${ }^{19}$ used a word list paradigm, the current study employed a pictorial task that lasted 18-min. Additionally, while being comparable in size, 
the study sample in Zoladz and colleagues ${ }^{19}$ included predominantly women while the current study only consisted of men. This could be important given that memory performance of men could be more affected by stress-induced cortisol elevations than that of women ${ }^{39}$.

We found that stress decreased the amount of recalled and correctly recognized neutral and negative pictures. Overall, studies that have investigated the effect of stress on memory encoding have yielded divergent findings, with some reporting enhanced subsequent memory performance ${ }^{40-43}$, others finding no effect ${ }^{11,44,45}$, impairing effects ${ }^{46-48}$, and still others reporting emotion dependent effects. For example, studies similar in design to the current study investigating pre-encoding stress in an incidental learning paradigm on delayed recall revealed an impairment of neutral information, while emotional information was enhanced ${ }^{49,50}$. It has been proposed that emotionally distressing situations might introduce a longer time window during which encoding of emotional information is not impaired ${ }^{17,43}$ which might be mediated by cortisol activation of the amygdala. Note that we did not obtain a generally better recognition performance for negative pictures than neutral pictures, while this emotional memory enhancement effect was observed for the free recal data. These results might appear to be conflicting and at odds with prior studies reporting an emotion memory enhancement effect. Yet, there are also examples of studies that did not find this emotional enhancement effect ${ }^{19,51,52}$. This effect may not have taken place in the present study because in general, the percentage correctly recognized old pictures was near ceiling irrespective of condition (i.e., $\mathrm{M}_{\mathrm{Hit}}>$ 94\%), which can have obscured emotion effects on recognition memory.

The exploratory ERP analyses revealed that stress 30-min before encoding decreased the P200 for negative and neutral pictures, while it increased the N200 for neutral pictures. It has been proposed that the amplitude of those two mid latency components reflect automatic post-perceptual selective attention ${ }^{53}$. A reduced P200 amplitude and an increased N200 amplitude has also been found in post-traumatic stress patients (PTSD) and in anxiety disorders in general ${ }^{54-57}$ reflecting the distorted attentional bias and hyper-arousal symptoms found in anxiety related disorders. The observation of an early modulation of the ERP by stress 30-min before encoding support the idea of sensory hyper-arousal under stress affecting attention-modulated information processing.

In line with previous research, the amplitude of the LPP in the 500-1000 ms window was larger for negative than for neutral pictures, known as the emotion effect ${ }^{23}$. Stress decreased the LPP in the 1500-2000 ms window for neutral pictures although irrespective of the timing of the stressor. Markedly, stress-induced cortisol responses (AUCi) were negatively associated with the amplitude of the LPP in the 500-1000 ms window within the distant stress group. Interestingly, the correct recognition of neutral pictures also correlated negatively with the AUCi in this distant stress group. It seems that stress 30-min before encoding decreased attentional allocation to neutral information thereby impairing the processing of new information. This observed antagonistic link on the behavioural and information processing level between the increase in cortisol and neutral information may mirror a refractory state of the hippocampus induced by genomic GR as well as non-genomic MR actions ${ }^{4,15,17,18}$. It is known that acute stress results in the release of neurotransmitters like noradrenaline in amongst others the prefrontal corte ${ }^{58}$. Based on animal and human work, it has been proposed that an interaction of cortisol with the noradrenergic system in the BLA is crucial for stress to affect emotional memory ${ }^{3,5}$. In line with this suggestion, we found a positive association between the AUCi and the amplitude of the LPP in the 500-1000 ms window for negative pictures within the proximate stress group. Our finding is also in accordance with Weymar and colleagues ${ }^{25}$, who found a selective enhancement of the LPP for unpleasant pictures in stressed participants suggesting that pre-learning stress tunes the brain for the processing of biologically relevant stimuli. Nevertheless, the present study did not replicate the finding of Weymar and colleagues ${ }^{25}$ regarding a positive correlation between the amplitude of the LPP of unpleasant pictures and memory performance 24-h later among stressed participants.

The fact that the timing of pre-learning stress did not directly affect attentional processing (i.e., LPP amplitude) or long term memory could indicate the need for a finer delineation of the precise time windows during which stress and glucocorticoids can affect memory encoding processes. For example, Diamond and colleagues ${ }^{15}$ found that predator stress immediately but not 30-min prior to learning in the water maze enhanced long-term memory in rats. Unfortunately, the task measurements used by Diamond and colleagues ${ }^{15}$ did not allow assessing the model based anticipated memory impairments when stress was applied 30-min before learning. Although animal research has been very valuable regarding the time dependent involvement of the MR and GR receptors to stress responses ${ }^{59}$, translation to humans seems to be intricate ${ }^{19,43}$.

Some limitations to the current study are worth mentioning. First, we did not measure activity of the sympathetic nervous system (e.g., via salivary alpha-amylase). 
However, it is very likely that by using emotionally arousing IAPS pictures, the current task also stimulated the adrenergic system including the amygdala ${ }^{60,61}$. In support of this interpretation, the used negative IAPS pictures were subjectively rated as arousing. Second, several studies involving acute psychological stressors have occasionally found evidence suggesting that sex differences may modulate the effects of cortisol on memory. Future studies using the current study design may benefit from including both men and women. Third, it would be interesting to investigate the effect of stress before encoding on the electrophysiological signature of remembered items, i.e., the subsequent memory effect ${ }^{23}$. This was not possible in the current study since 36 pictures per category were presented and the mean free recall percentage was $15 \%$, which clearly is an insufficient number of trials for ERP averaging. Future studies should take the low percentage of free recall into account, for instance by including more pictures in the encoding task. Moreover, the current study included moderately arousing unpleasant pictures. Effects of picture arousal on the LPP have consistently been found ${ }^{24}$ indicating the need to examine the effect of the proximity of stress on the encoding of high arousing stimuli. Finally, we have interpreted some of the findings based on high correlations found in relatively small samples. In order to further delineate the effect of timing of stress on memory, future work with larger sample sizes will need to be conducted.

In sum, the results of this study suggest that the time at which a stressor is applied might differentially impact the association between stress-induced cortisol elevations and memory formation for emotionally arousing and neutral events learned after the stressor. In this way, this study adds knowledge to the relatively sparse and inconsistent literature regarding the modulating effects of stress on memory formation. Administering stress prior to encoding seems a promising avenue to extend the temporal dynamics model of emotional memory processing ${ }^{15,18}$ that is supported by numerous studies demonstrating enhancing effects when stress is applied after learning during memory consolidation while stress applied before retrieval impairs memory. Thus, future studies should further delineate the exact time window under which memory is enhanced or impaired by acute psychosocial stress separately across different types of memory (e.g., emotional and neutral) and different memory paradigms (e.g., words, pictorial, incidental, and intentional), while carefully controlling for time of day and gender. Meanwhile, imaging techniques like EEG should be used since they seem to be more sensitive and can help to determine the underlying neurobiological mechanism.

\section{References}

1 de Kloet, E.R., Joels, M. \& Holsboer, F. (2005). Stress and the brain: from adaptation to disease. Nature Reviews: Neuroscience, 6, 463-475.

2 McGaugh, J.L. \& Roozendaal, B. (2002). Role of adrenal stress hormones in forming lasting memories in the brain. Current Opinion in Neurobiology, 12, 205-210.

3 Roozendaal, B., McEwen, B.S. \& Chattarji, S. (2009). Stress, memory and the amygdala. Nature Reviews: Neuroscience, 10, 423-433.

4 Joels, M., Fernandez, G. \& Roozendaal, B. (2011). Stress and emotional memory: a matter of timing. Trends in Cognitive Sciences, 15, 280-288.

5 Roozendaal, B. \& McGaugh, J. L. (2011). Memory modulation. Behavioral Neuroscience, 125, 797-824

6 LeDoux, J.E. (2000). Emotion circuits in the brain. Annual Review of Neuroscience, 23, 155-184.

7 Schwabe, L., Wolf, O.T. \& Oitzl, M.S. (2010). Memory formation under stress: quantity and quality. Neuroscience and Biobehavioral Reviews, 34, 584-591.

8 Wolf, O.T. (2009). Stress and memory in humans: twelve years of progress? Brain Research, 1293, 142-154.

9 Schwabe, L., Joels, M., Roozendaal, B., Wolf, O.T. \& Oitzl, M. S. (2012). Stress effects on memory: an update and integration. Neuroscience and Biobehavioral Reviews, 36, 1740-1749.

10 Cahill, L., Gorski, L. \& Le, K. (2003). Enhanced human memory consolidation with post-learning stress: interaction with the degree of arousal at encoding. Learning and Memory, 10, 270-274.

11 Smeets, T., Otgaar, H., Candel, I. \& Wolf, O.T. (2008). True or false? Memory is differentially affected by stress-induced cortisol elevations and sympathetic activity at consolidation and retrieval. Psychoneuroendocrinology, 33, 1378-1386.
12 de Quervain, D.J., Roozendaal, B. \& McGaugh, J.L. (1998). Stress and glucocorticoids impair retrieval of longterm spatial memory. Nature, 394, 787-790.

13 Kuhlmann, S., Piel, M. \& Wolf, O.T. (2005) mpaired memory retrieval after psychosocial stress in healthy young men. The Journal of neuroscience: the official journal of the Society for Neuroscience, 25 2977-2982.

14 Joels, M., Pu, Z.W., Wiegert, O., Oitzl, M. S. \& Krugers, H. J. (2006). Learning under stress: How does it work? Trends in Cognitive Sciences, 10, 152-158.

15 Diamond, D. M., Campbell, A.M., Park, C. R., Halonen, J. \& Zoladz, P.R. (2007). The temporal dynamics model of emotional memory processing: a synthesis on the neurobiological basis of stress-induced amnesia, flashbulb and traumatic memories, and the Yerkes-Dodson law. Neural Plasticity, 2007, 60803.

16 Joels, M., Karst, H., DeRijk, R. \& de Kloet, E.R. (2008). The coming out of the brain mineralocorticoid receptor. Trends in Neurosciences, 31, 1-7.

17 Karst, H., Berger, S., Erdmann, G., Schutz, G \& Joels, M. (2010). Metaplasticity of amygdalar responses to the stress hormone corticosterone. Proceedings of the National Academy of Sciences of the United States of America, 107, 14449-14454.

18 Joels, M., Pu, Z., Wiegert, O., Oitzl, M.S. \& Krugers, H.J. (2006). Learning under stress: how does it work? Trends in Cognitive Sciences, 10, 152-158.

19 Zoladz, P.R. et al. (2011). Pre-learning stress differentially affects long-term memory for emotional words, depending on temporal proximity to the learning experience. Physiology and Behavior, 103, 467-476. 
20 Hajcak, G., Weinberg, A., MacNamara., A, \& Foti, D. (2012). ERPs and the Study of Emotion. In Steven J. Luck \& Emily S. Kappenman (Eds), The Oxford Handbook of Event-Related Potential Components (pp 441-474). New York: Oxford University Press Inc.

21 Wilding, E.L. \& Ranganath, C. (2012) Electrophysiological Correlates of Episodic Memory Processes. In Steven J. Luck \& Emily S. Kappenman (Eds), The Oxford Handbook of Event-Related Potential Components (pp. 373-396). New York: Oxford University Press Inc

22 Cuthbert, B.N., Schupp, H. T., Bradley, M.M., Birbaumer, N. \& Lang, P.J. (2000). Brain potentials in affective picture processing: covariation with autonomic arousal and affective report. Biological Psychology, 52, 95-111.

23 Dolcos, F. \& Cabeza, R. (2002). Eventrelated potentials of emotional memory: encoding pleasant, unpleasant, and neutral pictures. Cognitive, Affective \& Behavioral Neuroscience, 2, 252-263.

24 Olofsson, J.K., Nordin, S., Sequeira, H. \& Polich, J. (2008). Affective picture processing: An integrative review of ERP findings. Biological Psychology, 77, 247-265.

25 Weymar, M., Schwabe, L., Low, A. \& Hamm, A.O. (2012). Stress sensitizes the brain: increased processing of unpleasant pictures after exposure to acute stress. Journal of Cognitive Neuroscience, 24, 1511 1518.

26 Roozendaal, B., Okuda, S., De Quervain D.J.F. \& McGaligh, J.L. (2006) Glucocorticoids interact with emotioninduced noradrenergic activation in influencing different memory functions. Neuroscience, 138, 901-910.
27 Smeets, T. et al. (2012). Introducing the Maastricht Acute Stress Test (MAST): A quick and non-invasive approach to elicit robust autonomic and glucocorticoid stress responses. Psychoneuroendocrinology, 37, 1998-2008.

28 Watson, D., Clark, L.A. \& Tellegen, A. (1988) Development and Validation of Brie Measures of Positive and Negative Affect the Panas Scales. Journal of Personality and Social Psychology, 54, 1063-1070.

29 Lang, P.J., Bradley, M.M. \& Cuthbert, B.N (2008). International Affective Picture System (IAPS): Affective Ratings of Pictures and Instruction Manual, Technical Report A-8.

30 Bradley, M.M. \& Lang, P.J. (1994). Measuring emotion: the Self-Assessment Manikin and the Semantic Differential. Journal of Behavior Therapy and Experimental Psychiatry, 25, 49-59.

31 Snodgrass, J.G. \& Corwin, J. (1988). Pragmatics of measuring recognition memory: applications to dementia and amnesia. Journal of Experimental Psychology-General, 117, 34-50.

32 Kudielka, B.M., Hellhammer, D.H. \& Kirschbaum, C. (2007). Ten Years of Research with the Trier Social Stress TestRevisited. In E. Harmon-Jones \& P. Winkielman (Eds.), Social neuroscience: Integrating biological and psychological explanations of social behavior (pp. 56-83). New York, NY, US: The Guilford Press.

33 Kirschbaum, C., Pirke, K.M. \& Hellhammer, D.H. (1993). The Trier Social Stress Test - A tool for investigating psychobiological stress responses in a laboratory setting. Neuropsychobiology, 28, 76-81.

34 Van Cauter, E. \& Refetoff, S. (1985). Evidence for two subtypes of Cushing's disease based on the analysis of episodic cortisol secretion. The New England journal of medicine, 312, 1343-1349.
35 Pruessner, J.C., Kirschbaum, C., Meinlschmid, G. \& Hellhammer, D.H. (2003). Two formulas for computation of the area under the curve represent measures of total hormone concentration versus timedependent

change. Psychoneuroendocrinology, 28, 916-931.

36 Fritz, C.O., Morris, P.E. \& Richler, J.J. (2012). Effect Size Estimates: Current Use, Calculations, and Interpretation. Journal of Experimental Psychology-General, 141, 2-18.

37 Domes, G., Heinrichs, M., Rimmele, U., Reichwald, U. \& Hautzinger, M. (2004). Acute stress impairs recognition for positive words--association with stressinduced cortisol secretion. Stress, 7, 173 181.

38 Qin, S., Hermans, E.J., van Marle, H.J. \& Fernandez, G. (2012). Understanding low reliability of memories for neutral information encoded under stress: alterations in memory-related activation in the hippocampus and midbrain. Journal of Neuroscience, 32, 4032-4041.

39 Wolf, O.T., Schommer, N.C., Hellhammer, D.H., McEwen, B.S. \& Kirschbaum, C. (2001). The relationship between stress induced cortisol levels and memory differs between men and women Psychoneuroendocrinology, 26, 711-720.

40 Kuhlmann, S. \& Wolf, O.T. (2006). Arousa and cortisol interact in modulating memory consolidation in healthy young men. Behavioral Neuroscience, 120, $217-$ 223

41 Nater, U.M. et al. (2007). Performance on a declarative memory task is better in high than low cortisol responders to psychosocial stress. Psychoneuroendocrinology, 32, 758-763.
42 Schwabe, L., Bohringer, A., Chatterjee, M. \& Schachinger, H. (2008). Effects of prelearning stress on memory for neutral positive and negative words: Differen roles of cortisol and autonomic arousal. Neurobiology of Learning and Memory, 90, 44-53.

43 Smeets, T. et al. (2009). Stress selectively and lastingly promotes learning of contextrelated high arousing information Psychoneuroendocrinology, 34, 1152-1161.

44 de Quervain, D.J., Roozendaal, B., Nitsch, R.M., McGaugh, J.L. \& Hock, C. (2000). Acute cortisone administration impairs retrieval of long-term declarative memory in humans. Nature Neuroscience, 3, 313-314.

45 Domes, G., Heinrichs, A., Reichwald, U. \& Hautzinger, M. (2002). Hypothalamicpituitary-adrenal axis reactivity to psychological stress and memory in middle-aged women: high responders exhibit enhanced declarative memory performance. Psychoneuroendocrinology, 27, 843-853.

46 Kirschbaum, C., Wolf, O.T., May, M. Wippich, W. \& Hellhammer, D.H. (1996). Stress- and treatment-induced elevations of cortisol levels associated with impaired declarative memory in healthy adults. Life Sciences, 58, 1475-1483.

47 Lupien, S.J. et al. (1997). Stress-induced declarative memory impairment in healthy elderly subjects: relationship to cortisol reactivity. The Journal of Clinical Endocrinology and Metabolism, 82, 20702075.

48 Kim, J.J., Lee, H.J.J., Han, J.S. \& Packard, M. G. (2001). Amygdala is critical for stressinduced modulation of hippocampal longterm potentiation and learning. Journal of Neuroscience, 21, 5222-5228.

49 Payne, J.D. et al. (2006). The impact of stress on neutral and emotional aspects of episodic memory. Memory, 14, 1-16. 
50 Payne, J.D. et al. (2007). Stress administered prior to encoding impairs neutral but enhances emotional long-term episodic memories. Learning and Memory, 14, 861 868.

51 Schwabe, L. \& Wolf, O.T. (2010). Learning under stress impairs memory formation. Neurobiology of Learning and Memory, 93, 183-188.

52 Zoladz, P.R. et al. (2013). Pre-learning stress that is temporally removed from acquisition exerts sex-specific effects on long-term memory. Neurobiology of Learning and Memory, 100, 77-87.

53 Luck, S.J. (2005). An Introduction to the Event-related Potential Technique. The MIT Press.

54 Felmingham, K.L., Tran, T.P., Fong, W.C. \& Bryant, R.A. (2012). Sex differences in emotional memory consolidation: The effect of stress-induced salivary alphaamylase and cortisol. Biological Psychology, 89, 539-544.

55 Wessa, M., Karl, A. \& Flor, H. (2005). Central and peripheral psychophysiological responses to trauma-related cues in subclinical posttraumatic stress disorder: a pilot study. Experimental Brain Research, 167, 56-65.

56 Sass, S.M. et al. (2010). Time course of attentional bias in anxiety: Emotion and gender specificity. Psychophysiology, 47, 247-259.

57 Javanbakht, A., Liberzon, I., Amirsadri, A Gjini, K. \& Boutros, N.N. (2011). Eventrelated potential studies of post-traumatic stress disorder: a critical review and synthesis. Biology of Mood \& Anxiety Disorders, 1, 5.

58 Arnsten, A.F. (2009). Stress signalling pathways that impair prefrontal cortex structure and function. Nature Reviews: Neuroscience, 10, 410-422.
59 de Kloet, E.R. (2008). About stress hormones and resilience to psychopathology. Journal of Neuroendocrinology, 20, 885-892.

60 Phan, K.L., Wager, T., Taylor, S.F. \& Liberzon I. (2002). Functional neuroanatomy of emotion: A meta-analysis of emotion activation studies in PET and FMRI. Neuroimage, 16, 331-348.

61 van Stegeren, A.H., Roozendaal, B., Kindt M., Wolf, O.T. \& Joels, M. (2010). Interacting noradrenergic and corticosteroid systems shift human brain activation pattern during encoding. Neurobiology of Learning and Memory, 93, 56-65.

\section{Appendix}

IAPS numbers of the 36 negative and 36 neutral pictures used in the encoding task

Neutral:

$\begin{array}{lllllllllll}2392 & 2489 & 2362 & 2102 & 2383 & 2377 & 2019 & 7506 & 2002 & 2374 & 7235 \\ 7211 & 7546 & 7056 & 7055 & 7530 & 7081 & 7001 & 7016 & 7018 & 7492 & 5870\end{array}$ $\begin{array}{llllllllllll}7037 & 5829 & 7580 & 7499 & 7165 & 5635 & 7509 & 7512 & 7505 & 7014 & 1603\end{array}$ 161016701500

Negative:

$\begin{array}{lllllllllll}9250 & 3053 & 6021 & 6571 & 6550 & 3001 & 2710 & 9413 & 9921 & 6212 & 9440\end{array}$ $\begin{array}{lllllllllll}2682 & 6263 & 9301 & 9600 & 9409 & 9622 & 3005,1 & 2692 & 7361 & 9911 & 9001\end{array}$ $\begin{array}{lllllllllll}9291 & 5970 & 3213 & 9050 & 9253 & 6010 & 9630 & 9592 & 2799 & 6200 & 1300\end{array}$

$1050 \quad 9181 \quad 1930$

IAPS numbers of the 36 negative and 36 neutral pictures used in the recognition task.

Negative similar:

$\begin{array}{lllllllllll}9410 & 3051 & 6022 & 6821 & 6540 & 9480 & 2681 & 6260 & 9300 & 9620 & 9903\end{array}$ $\begin{array}{lllllll}9000 & 9295 & 5973 & 8485 & 9594 & 1525 & 1052 .\end{array}$

Negative different:

$\begin{array}{lllllllllll}2206 & 9800 & 9325 & 2345,1 & 3185 & 9008 & 9010 & 9006 & 6300 & 9810 & 9940\end{array}$ $\begin{array}{lllllll}5950 & 8230 & 6312 & 2751 & 7079 & 9180 & 1201\end{array}$

Neutral similar:

$\begin{array}{lllllllllll}2391 & 2488 & 2332 & 2570 & 2381 & 7026 & 7190 & 7547 & 7034 & 7236 & 7489\end{array}$ $\begin{array}{lllllll}5982 & 7039 & 5830 & 7507 & 2840 & 1602 & 1750 .\end{array}$

Neutral different:

$\begin{array}{lllllllllll}2394 & 2530 & 2410 & 7550 & 2191 & 7950 & 7900 & 5390 & 7233 & 7080 & 2594\end{array}$

$\begin{array}{llllllll}7501 & 7620 & 7036 & 7000 & 2032 & 1740 & 1720 .\end{array}$ 


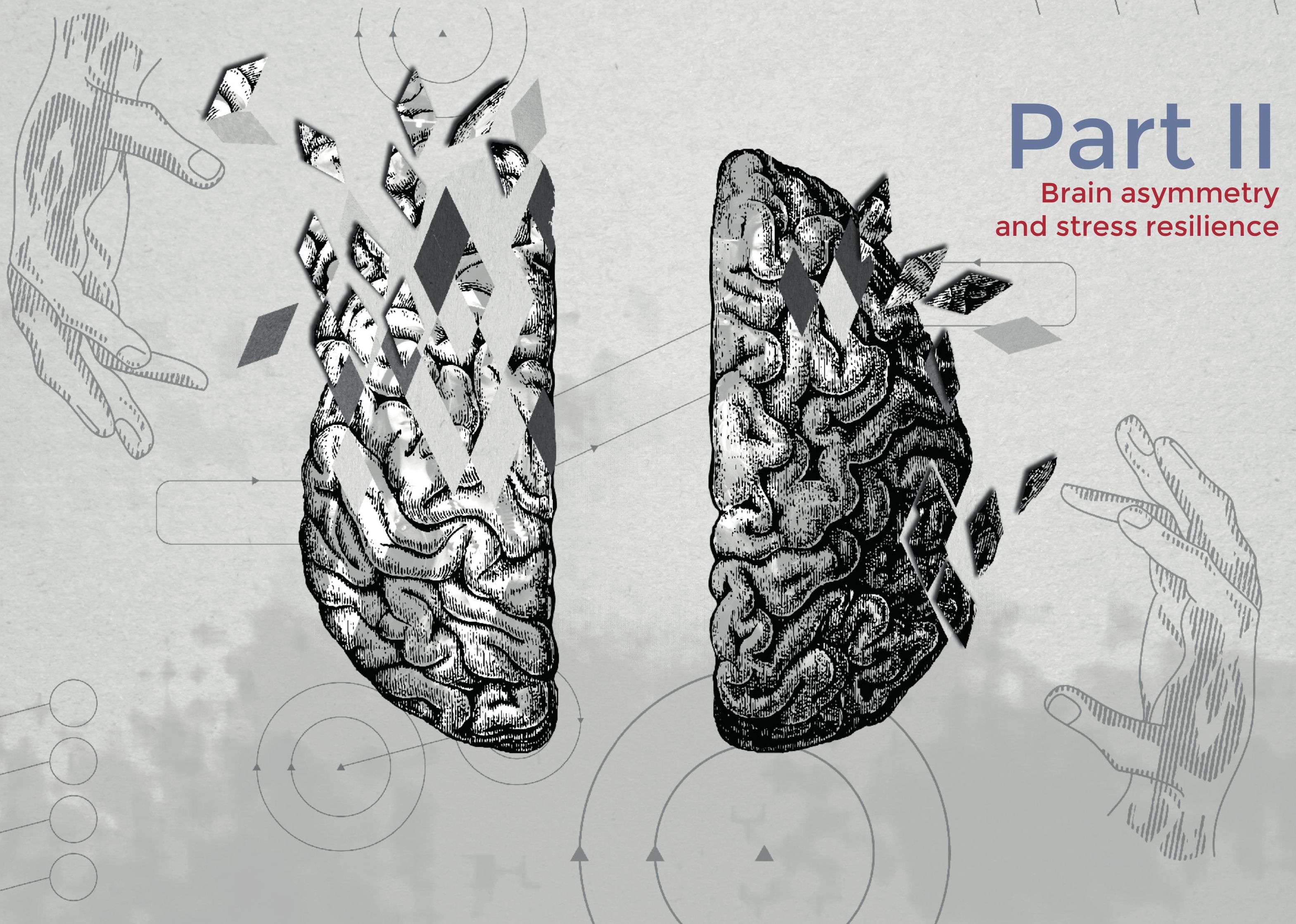




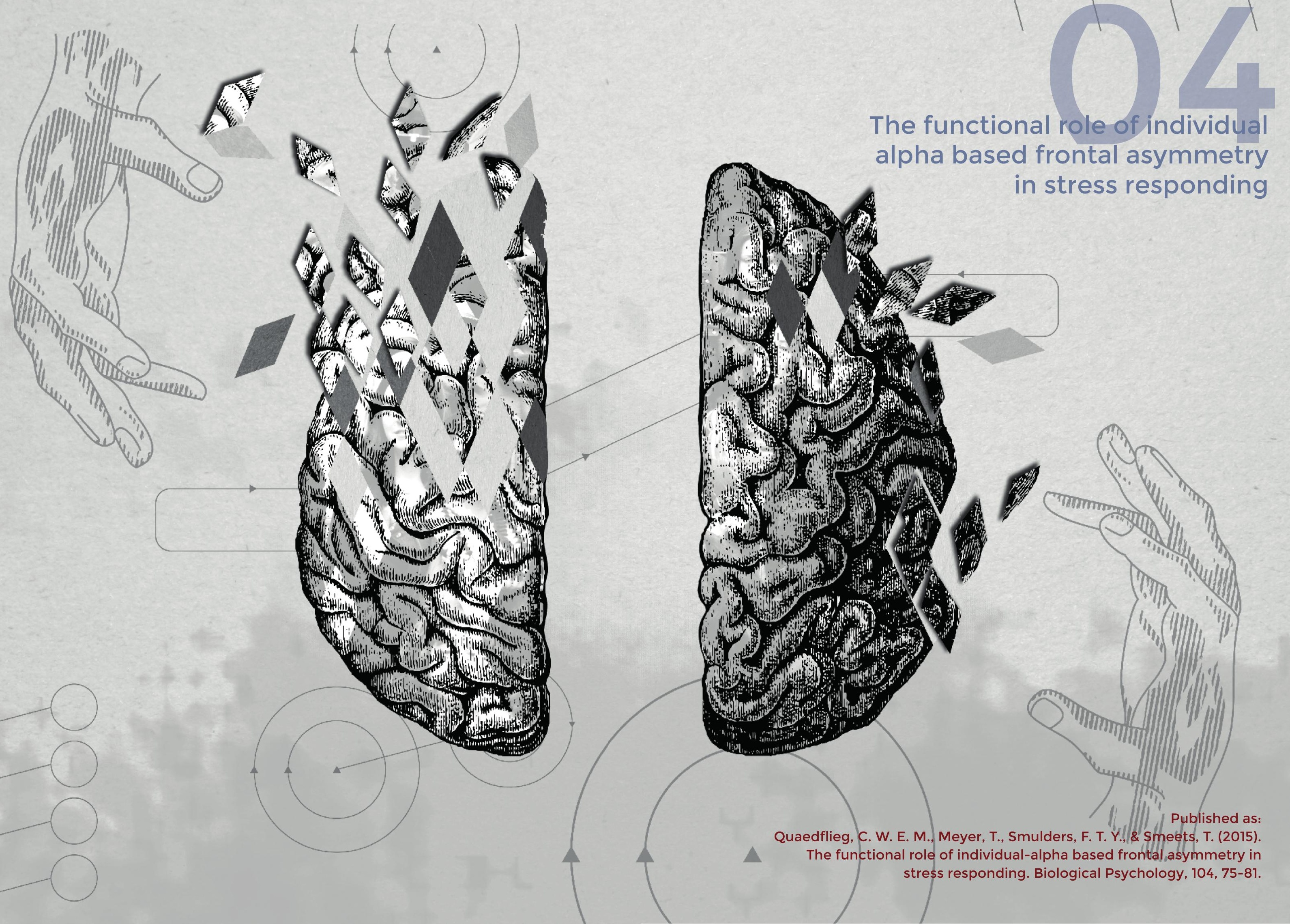




\section{Abstract}

Asymmetry in frontal electrical activity has been suggested to index tendencies in affective responding and thus may be associated with hormonal stress responses. To assess the functional role of frontal asymmetry (FA) in stress, we measured FA at rest and following exposure to acute stress induced with the Maastricht Acute Stress Task (MAST; N=70) in the standard $8-13 \mathrm{~Hz}$ band as well as based on individual alpha frequency (IAF) band. IAF-based resting $\mathrm{FA}_{\mathrm{F} 4-\mathrm{F} 3}$ was associated with the stress-induced neuroendocrine response, such that left individual frontal activity predicted smaller total cortisol increases in response to the MAST. Like previous studies, we found resting left-sided $F A_{F 8-F 7}$ to predict trait behavioural activation measured with the BIS/BAS scales. FA remained unaffected by stress-induced cortisol response. These findings suggest that individual FA might reflect a trait-like characteristic that moderates the stress response. Our results underscore the utility of IAF in studying individual differences in stress responding.

Keywords: frontal alpha oscillations, individual alpha frequency, cortisol, EEG 
Research suggests that a functional lateralization in the prefrontal cortex is involved in affective processing ${ }^{1-3}$. A reliable correlate of frontal activity is frontal asymmetry (FA) measured with electroencephalography (EEG). FA refers to the average difference in alphaband activity (typically $8-13 \mathrm{~Hz}$ ) between the left and right frontal areas across several minutes. FA measured while participants are at rest has been regarded as an index of individuals' trait-like style of affective processing, whereas FA measured during or after emotional challenge has been linked to state-dependent individual differences in affective processing $^{1,4-7}$. For instance, more left-sided resting FA (i.e., higher activity in the left frontal areas) has been linked to superior emotional flexibility ${ }^{8}$, more effective emotion regulation ${ }^{9}$ as well as to more positive and decreased negative affect ${ }^{10}$. Furthermore, an association between right-sided FA and higher basal cortisol levels has been found in animal studies at baseline ${ }^{11}$ and after maternal separation ${ }^{12}$, as well as in human studies in six-month-old infants at baseline and during a withdrawal task ${ }^{13}$, and in students during a stressful exam period $^{14}$. While these findings suggest that resting FA may be predictive of stress hormonal responses, other lines of research indicate that the stress response itself might involve lateralized processes in the brain. That is, animal and human studies suggest that the right hemisphere initiates neuroendocrine and behavioural fight-or-flight responses, while the left hemisphere regulates them ${ }^{15-20}$. Right-sided FA at rest and in response to stress could thus be expected to predict stronger hormonal stress responses. However, to date, only three experimental studies have examined the relationship between FA, stress, and stress hormonal responses, and have yielded mixed results. Tops and colleagues ${ }^{21}$ found that the administration of cortisol shifted the relative FA to the right. In a follow-up study, however, these authors found that cortisol administration in a formal and arousing testing condition shifted FA to the left ${ }^{22}$. Alternatively, Lewis, Weekes, and Wang ${ }^{23}$ found that high in comparison to low examination stress increased relative right-sided FA activity.

Previous research has shown that alpha frequency varies in peak and bandwidth from individual to individual ${ }^{24-27}$. Moreover, individual differences in alpha peak frequency have been related to several cognitive functions including perception, attention, and memory ${ }^{28,29}$. Thus, the use of fixed frequency bands in previous studies may have obscured information about specific individual differences in neurophysiology of the brain. Using individual alpha frequency bands could potentially improve the signal-to-noise ratio and thus improve reliability of FA measurements.
In the current study, we used the Maastricht Acute Stress Task (MAST) ${ }^{30}$ to induce neuroendocrine stress responses and investigated the functional role of asymmetric frontal alpha oscillations. Based on previous studies, we hypothesized that more right-sided FA at rest and in response to stress, would be related to higher cortisol increases in response to the MAST. Furthermore, we expected that FA at rest would be associated with level of trait motivation. The second aim of the study was to examine whether FA based on an individualized alpha frequency band can provide a more sensitive measure of individual differences in lateralized stress responding.

\section{Methods}

\section{Participants}

The present experiment was part of a larger study that investigated frontal alpha asymmetry and resilience. Right-handed healthy men $(n=30)$ and women $(n=40)$ undergraduates (mean age: 20.83 SD 2.67; range: 18-31 years) were recruited via advertisements at Maastricht University. Participants were screened for eligibility using the following exclusion criteria: history of psychiatric, neurologic, cardiovascular or neuroendocrine diseases, heavy smoking (i.e., more than 15 cigarettes/day), medication use known to affect the autonomic nervous system (ANS) or hypothalamic-pituitary-adrenal (HPA) axis, drug use, or body mass index outside the normal range. Moreover, for women, the use of oral contraceptives served as an inclusion criterion to reduce variability in cortisol responses related to hormonal alterations throughout the menstrual cycle phase ${ }^{31}$. Test protocols were approved by the standing ethics committee of the Faculty of Psychology and Neuroscience, Maastricht University. All participants signed a written informed consent and were given a small reward (course credits or money) in return for their participation.

\section{Procedure}

An overview of the experimental session is displayed in Fig. 4.1. All testing took place between 12:30 and 18:00-h to avoid morning fluctuations in the circadian rhythm of cortisol and time-of-day effects on frontal asymmetry ${ }^{32}$. Beforehand, participants were asked via email to refrain from eating, exercising extensively or drinking anything but non-sparkling water for 2-h prior to the experimental session. Upon arrival in the laboratory, participants 
received information on the experimental procedure and gave written consent. Next, a saliva sample was taken and participants were told that this sample would be immediately assayed to check their adherence to the instructions not to eat, drink, etc. This was done to increase truth-telling behaviour. In reality, the sample was discarded without being analysed. Participants were seated in front of a 22-inch widescreen monitor (Philips, The Netherlands) at approximately $56 \mathrm{~cm}$ viewing distance and drank $200 \mathrm{ml}$ of apple juice to standardize glucose levels ${ }^{33}$.

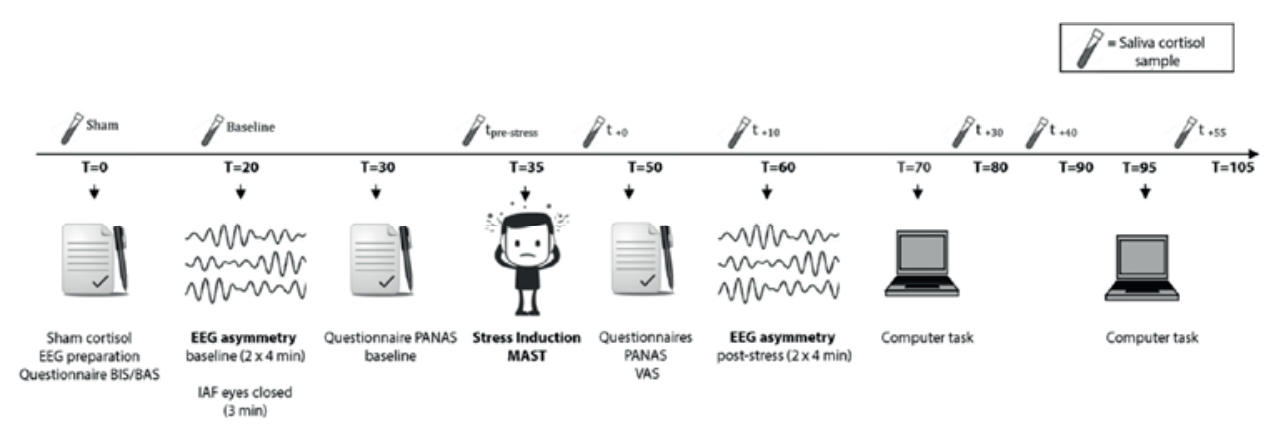

Figure 4.1 Overview of the study procedure. $T_{+0}$ refers to the end of the stress procedure. Abbreviations: IAF: Individual Alpha Frequency, MAST: Maastricht Acute Stress Test.

\section{Stress manipulation}

The Maastricht Acute Stress Test (MAST) ${ }^{30}$ consists of a 5 -min preparation phase in which the task is explained and a 10-min acute stress phase that includes several exposures to cold pressor stress and various mental arithmetic challenges along with social-evaluative pressure (i.e., negative feedback). Specifically, in five trials that varied in duration from 60 to $90-s$, participants immersed their hand into ice-cold water $\left(2^{\circ} \mathrm{C}\right.$; plexiglas box with an electrical cooler and a circulation pump from JULABO Labortechnik, Seelbach, Germany). In between the hand immersion trials, participants engaged in mental arithmetic challenges in which they had to count backwards as fast and accurately as possible in steps of 17 starting at 2043 for 45,60 , or 90 -s. Whenever they counted too slowly or made a mistake, they received negative feedback (i.e., to count faster or start over again at 2043). To increase unpredictability and uncontrollability, participants were told that the order and duration of the hand immersion and mental arithmetic trials would be randomly chosen by the computer and that they would be videotaped.

\section{Neuroendocrine stress responses}

Cortisol values prior to and in response to the MAST were obtained with synthetic Salivettes (Sarstedt ${ }^{\circ}$, Etten-Leur, The Netherlands). Participants provided saliva samples 20-min after arrival in the lab ( $\left.t_{\text {base }}\right), 5$-min before ( $\left.t_{\text {pre-stress }}\right)$ the MAST and five times afterwards $\left(t_{+0,}, t_{+10}, t_{+30}\right.$ $t_{+40}, t_{+55 \min }$ with reference to the end of the stressor). Samples were stored at $-20^{\circ} \mathrm{C}$ until cortisol levels were determined by a commercially available luminescence immune assay kit (IBL, Hamburg, Germany). Mean intra- and inter-assay coefficients of variation are typically less than $5 \%$, and the lower and upper detection limits were $0.015 \mathrm{mg} / \mathrm{dl}(0.41 \mathrm{nmol} / \mathrm{l})$ and $4.0 \mathrm{mg} / \mathrm{dl}(110.4 \mathrm{nmol} / \mathrm{l})$, respectively. One woman did not provide enough saliva to be analysed. Thus, the final sample consisted of 69 participants.

Cortisol after arrival in the lab ( $\left.t_{\text {base }}\right)$ and before the MAST ( $\left.t_{\text {pre-stress }}\right)$ did not differ significantly $\left(F_{(1,64)}=1.91, p=.17\right)$. For the correlations, the Area Under the Curve with respect to increase (AUCi) from the pre-stress sample was calculated as a single measure of the total cortisol concentration in response to the MAST for each participant individually ${ }^{34}$.

\section{Approach behaviour}

\section{Behavioural inhibition and activation system scales ${ }^{35}$}

The BIS/BAS Scales were used to assess a person's disposition towards the two motivational systems i.e. approach and withdrawal. The questionnaire consists of 22 items assessing behavioural inhibition (BIS; 7 items) and behavioural activation (BAS; 13 items). The BAS is further divided into three subscales: fun seeking (BAS-F; 4 items), reward responsiveness (BAS-R; 5 items), and drive (BAS-D; 4 items). Participants answer the extent to which they agree with the statements on a four-point Likert-type scale, ranging from 1 (strong agreement) to 4 (strong disagreement). Higher scores relate to higher BIS/BAS sensitivity.

\section{EEG data acquisition and analysis}

The electroencephalogram (EEG) was recorded from $23 \mathrm{Ag} / \mathrm{AgCl}$ electrodes (F7, F3, Fz, F4, F8, FC3, FC4, T7, T8, C3, Cz, C4, CP3, CPz, CP4, P7, P3, Pz, P4, P8, O1, Oz, O2) positioned in an elastic cap according to the international 10-20 system using a BrainAmp amplifier and Brain Recorder software (BrainProducts, Germany). Signals were sampled continuously at $100 \mathrm{~Hz}$, referenced online to the left mastoid (A1) and band-pass filtered $(0.01-30 \mathrm{~Hz})$. An electrode at AFz served as signal ground. Two electrodes at the outer canthi of both eyes recorded 
horizontal eye movements and two electrodes above and below the left eye recorded vertical eye movements. Scalp-electrode impedance was kept below $5 \mathrm{k} \Omega$ to ensure highquality EEG recordings and homologous scalp electrodes were within $1 \mathrm{k} \Omega$ of each other. Participants were shown the raw recording signals to demonstrate common artefacts that occur due to body and eye movements. FA was measured before and after stress induction during two 4-min blocks, whereby participants focused on a black fixation cross on grey background on the computer monitor.

Offline analyses were performed with Vision Analyzer 2.0 (Brain Products, Germany). Consistent with data reduction procedures in previous FA studies ${ }^{36,37}$, the data was rereferenced offline to the average of $A 1$ and $A 2$, band-pass filtered from 1 to $30 \mathrm{~Hz}$ and corrected for EOG activity using an algorithm similar to Gratton and Coles $^{38}$. To derive resting-state FA scores, 2-s epochs with 75\% overlap were extracted. Epochs containing EEG changes exceeding $\pm 75 \mu \mathrm{V}$ were automatically omitted from averages. On average 955.61 $($ range $=895-1005 ;$ SEM $=2.49)$ of baseline and 963.67 (range $=913-1020 ;$ SEM $=2.10)$ of post stress epochs were artefact free. Artefact-free epochs were analysed using fastFourier transformation (FFT) with a 100\% Hanning window to compute power density values. Average power densities of the two 4-min resting state measurements were calculated and weighted for the number of artefact-free epochs for both the baseline and the post-stress measurement.

The individual alpha peak frequency (IAF) was determined as the dominant frequency rhythm between 5 and $15 \mathrm{~Hz}$ at the posterior electrode (Pz) on 3-min of resting eyes closed data ${ }^{25,29}$. The IAF bandwidth was defined as the IAF $\pm 0.20 \times$ IAF. The frontal asymmetry (FA) scores were determined in the IAF band and in the standard alpha band between 8 and $13 \mathrm{~Hz}$ and were calculated on log-transformed alpha-power density values, $\ln ($ right) $-\ln$ (left). Positive frontal alpha asymmetry scores indicate greater left than right frontal activity since alpha band power is inversely related to brain activity ${ }^{39}$.

\section{Data analysis}

Cortisol data were log-transformed before analysis as Shapiro-Wilk tests of normality showed typical positive skewness of the data. Effectiveness of the stress induction procedure on neuroendocrine measures including differences between men and women were addressed using repeated measures ANOVA with time ( 6 levels: $t_{\text {pre-stress }}, t_{+0}, t_{+10}, t_{+30}, t_{+40}$, $\left.t_{+55 \min }\right)$ as within-subject variable and gender as between-subject variable. Effects of the stress on FA as well as differences between men and women were addressed using repeated measures ANOVA with manipulation (baseline, post-stress) and location (F4-F3, F8-F7) as within subject variables and gender as between subject variable. To explore possible relationships between participants' physiological stress response (i.e., AUCi) and brain asymmetry, bivariate Pearson correlations were conducted. We used a hypothesis driven approach (i.e., including asymmetry scores at frontal electrodes only) to limit the inflation of Type I error rates in these analyses and corrected for multiple testing (i.e., F4-F3 and F8-F7) by using a significance level of $p \leq .025$. When sphericity assumptions for ANOVAs were violated, Greenhouse-Geisser corrected $p$-values, along with the respective epsilon and uncorrected degrees of freedom are reported.

\section{Results}

\section{Stress manipulation: neuroendocrine responses}

Mean cortisol concentrations prior to and following the MAST are shown in Fig. 4.2. Repeated measures ANOVAs revealed a significant time $x$ gender interaction $\left(F_{(5,310)}=5.09\right.$, $p=.004$, Epsilon $=.48$ ). Follow-up analyses per gender revealed a main effect of time (six levels: $\mathrm{t}_{\text {prestress }}, \mathrm{t}_{+0,} \mathrm{t}_{+10,} \mathrm{t}_{+30}, \mathrm{t}_{+40,} \mathrm{t}_{+55 \mathrm{~min}}$ men: $F_{(5,130)}=22.84, p<.001$, Epsilon $=.44$; women: $F_{(5,180)}$ $=8.14, p<.001$, Epsilon $=.44)$. Bonferroni corrected simple effects per saliva time point revealed a significant increase between $\mathrm{t}_{\text {pre-stress }}$ and $\mathrm{t}_{+10}$ (men: $p<.001$; women: $p=.01$ ) and a significant decrease between $\mathrm{t}_{+30}$ and $\mathrm{t}_{+40}$ (men: $p<.001$; women: $p<.001$ ) to a return to baseline at the end of the experiment ( $\mathrm{t}_{\text {prestress }}$ vs $\mathrm{t}_{+55}$ men: $p>.99$; women: $p>.99$ ).

\section{Frontal asymmetry: standard versus individual alpha}

The mean of the IAF was $9.98 \mathrm{~Hz}($ SEM .11; minimum $=7.4$ maximum $=11.3)$ and was not statistically different from the centre $(10 \mathrm{~Hz})$ of the standard alpha band $\left(F_{(1,50)}=0.05, p=.82\right)$. Bivariate Pearson correlations were calculated between the first and second block of the baseline $\mathrm{FA}_{\mathrm{F} 4 \mathrm{~F} 3}$ for the standard and IAF band separately to evaluate whether IAF-based FA yielded higher (test-retest) reliability scores than the standard $8-13 \mathrm{~Hz}$ alpha band. The standard alpha $(8-13 \mathrm{~Hz})$ based baseline $\mathrm{FA}_{\mathrm{F} 4 \mathrm{~F} 3}$ correlation between the two 4-min blocks was $r_{67}=.70$ and the IAF-based FA correlation was $r_{67}=.85$. These correlations were 
significantly different (baseline: $Z=-2.22, p=.026$, two-tailed) indicating a better test-retest stability for IAF-based $\mathrm{FA}_{\mathrm{F} 4 \mathrm{~F} 3}$.

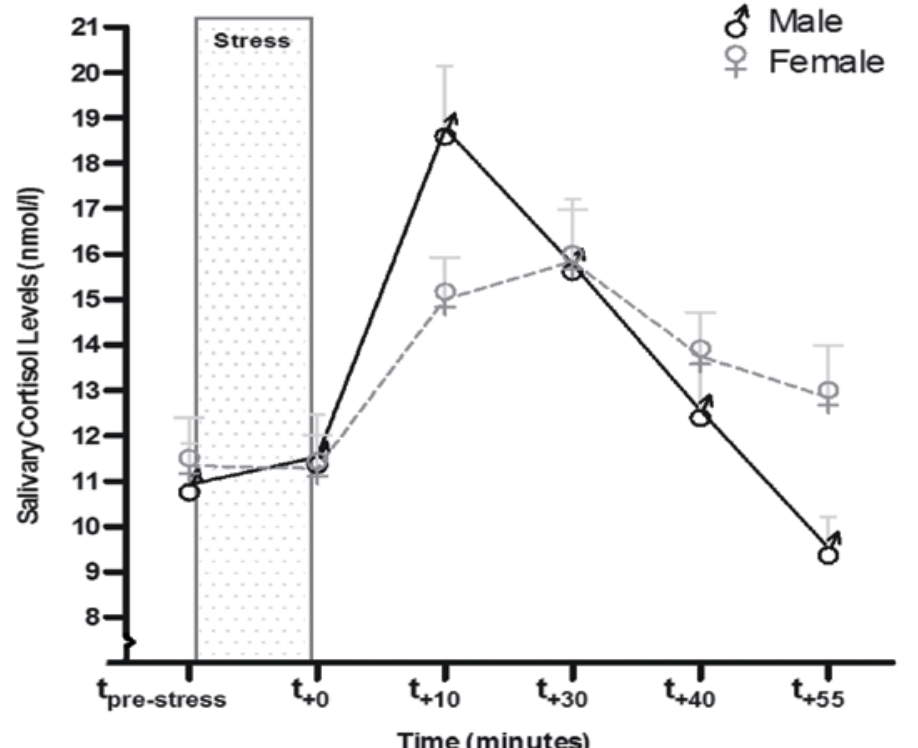

Figure 4.2 Salivary cortisol responses to the Maastricht Acute Stress Test (MAST). Graphs show means $\pm \mathrm{SE}$.

\section{Frontal asymmetry and stress induction}

For the mean power density values based on standard alpha band $(8-13 \mathrm{~Hz})$, repeated measures ANOVA with manipulation (baseline, post-stress) and location (F4-F3, F8-F7) as within subject variables and gender as between subject variable revealed no significant interactions and only a main effect of location $\left(F_{(1,67)}=25.84, p<.001\right)$. Likewise, for the IAFbased $F A$, no significant interactions and only a main effect of location $\left(F_{(1,67)}=25.69, p<.001\right)$ was found, indicating that stress did not directly affect frontal asymmetry' (see Fig. 4.3).

Hemisphere specific stress effects were assessed using a MANOVA with manipulation (baseline, post-stress) and hemisphere (left, right) as within subject variables and gender as between subject variable. No manipulation $x$ hemisphere interaction was found $\left(F_{(1,6)}=2.58, p=.12\right)$.

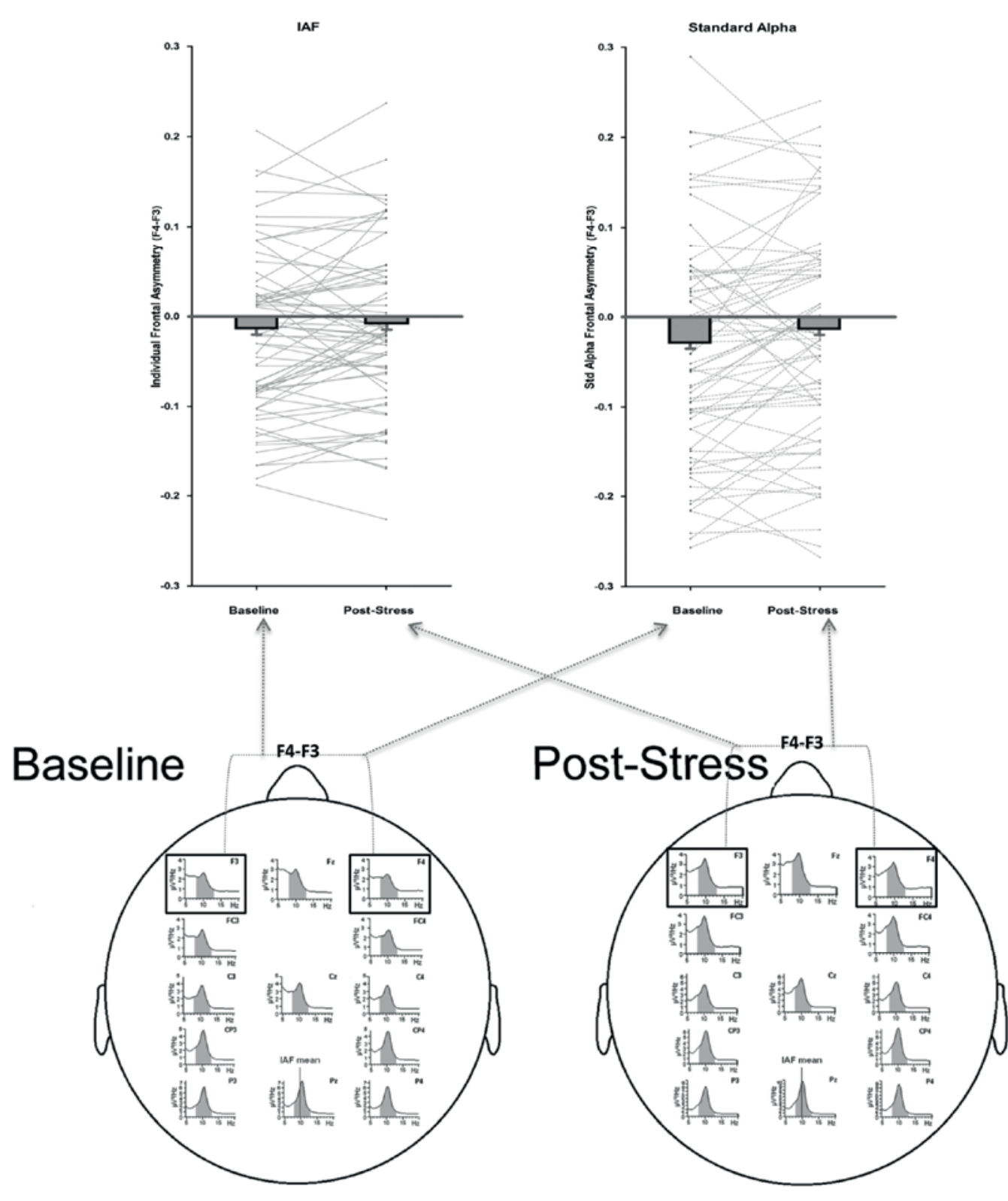

Figure 4.3. Effect of stress induction on alpha oscillations. Upper panel: Effect of stress induction on frontal alpha asymmetry across the whole sample (mean plus std. error) as well as per individual (lines) for individual (left) and standard (right) alpha band. Lower panel: Topographical display of average power density at rest before and after the stress induction. The grey area marks $8-13 \mathrm{~Hz}$, in which power densities were averaged to derive alpha asymmetry scores in the standard alpha band. The red line at Pz marks the mean IAF. Note the difference in Y-axis scaling between frontal and parietal electrodes. 


\section{Frontal asymmetry, neuroendocrine response, and behavioural activation}

Bivariate Pearson correlations between participants' physiological stress response and brain asymmetry were computed. Table 4.1 displays the correlations between total cortiso concentration (AUCi) and the standard alpha band $(8-13 \mathrm{~Hz})$ and IAF for baseline, post-stress

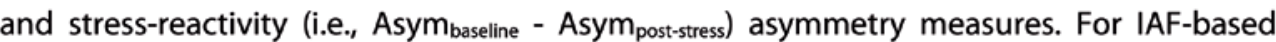
baseline F4-F3 asymmetry, a negative correlation $\left(r_{67}=-.27 ; p=.025\right)$ was found, showing that more left-sided frontal activity at baseline was associated with a smaller cortiso response to the stressor (see Fig. 4.4). There was also a negative association with $\mathrm{FA}_{\mathrm{F} 4 \mathrm{~F} 3}$ poststress based on IAF $\left(r_{67}=-.24 ; p=.05\right)$. However, the associations with $\mathrm{FA}_{\mathrm{F} 4 \mathrm{~F} 3}$ baseline and post-stress based on standard alpha remained non-significant (baseline: $r_{67}=-.21 ; p=.08$; post-stress: $r_{67}=-.20 ; p=.10$ ). Moreover, the IAF associations for baseline and post-stress FA were specific for the mid-frontal electrodes (F4-F3) since all other correlations were nonsignificant (all ps $>$.78). Stress-reactivity FA did not correlate significantly with the cortisol response, for both standard alpha and IAF (all ps > .68, see Table 4.1)"

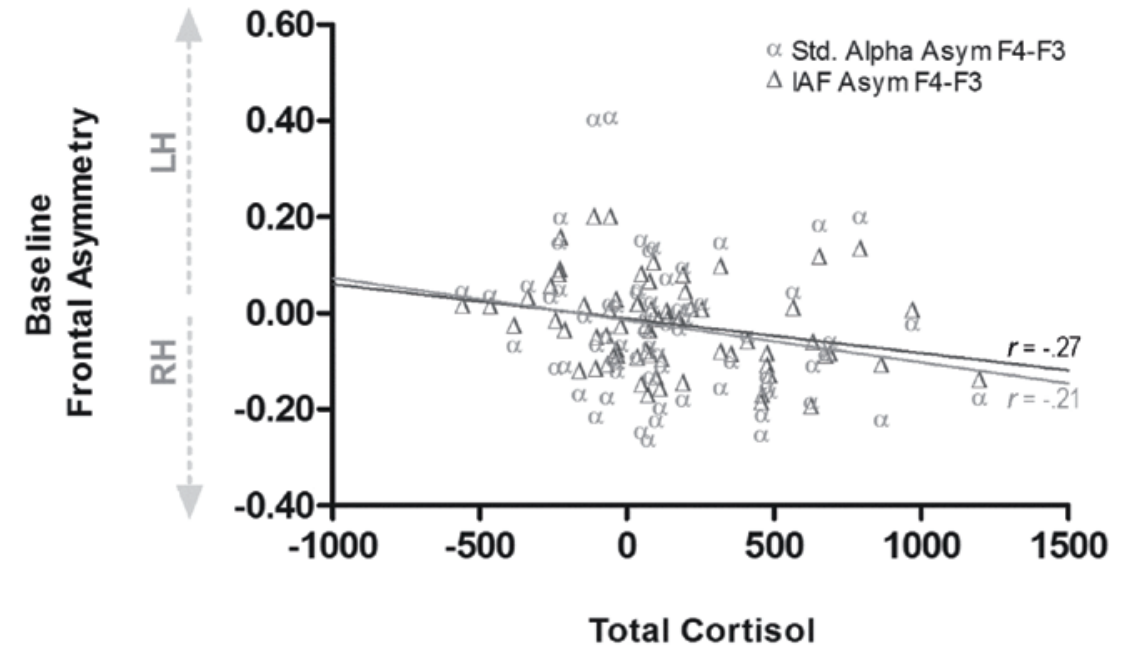

Figure 4.4. Association between baseline asymmetry at $\mathrm{F4}-\mathrm{F} 3$ and total cortisol response. More right-sided asymmetry resulted in a higher cortisol increase. Note that the correlation was only significant for IAF.

"Bivariate Pearson correlations between the cortisol response immediately before the frontal asymmetry measurement (i.e., $\left.t_{+10}\right)$ and brain asymmetry were nearly identical (IAF: $r_{69}=-.25 ; p=.04 ;$ std. alpha $r_{69}=-.22 ; p$ $=.08)$.
Table 4.1 Summary of the EEG results. Upper part: Mean \pm S.E.M. of the frontal asymmetry before and after stress induction for $\mathrm{F} 4 \mathrm{~F} 3$ and $\mathrm{F} 8-\mathrm{F} 7$ separately based on $8-13 \mathrm{~Hz}$ or IAF. Lower part associations between the cortisol response and brain asymmetry based on 8-13Hz or IAF.

\begin{tabular}{|c|c|c|c|c|c|}
\hline & & \multicolumn{2}{|c|}{ Standard Alpha 8-13Hz } & \multicolumn{2}{|c|}{ Individual Alpha } \\
\hline & & Mean & SEM & Mean & SEM \\
\hline \multirow{2}{*}{ Baseline } & Asym $_{\text {F4-F3 }}$ & -0.024 & 0.018 & -0.022 & 0.011 \\
\hline & Asym $_{F 8-F 7}$ & -0.186 & 0.037 & -0.118 & 0.024 \\
\hline \multirow{2}{*}{$\begin{array}{l}\text { Post- } \\
\text { stress }\end{array}$} & Asym $_{F 4-F 3}$ & -0.010 & 0.017 & -0.012 & 0.011 \\
\hline & Asym $_{\text {F8-F7 }}$ & -0.159 & 0.034 & -0.112 & 0.023 \\
\hline
\end{tabular}

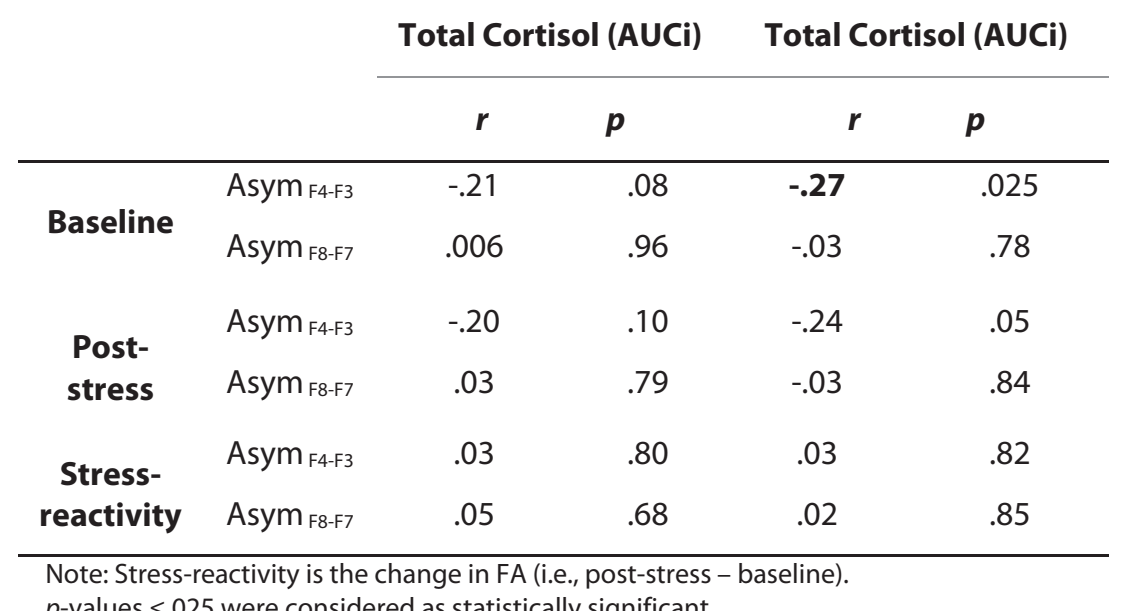

$p$-values $\leq 025$ were considered as statistically significant.

Measures of behavioural activation and inhibition (BIS/BAS) were correlated with brain

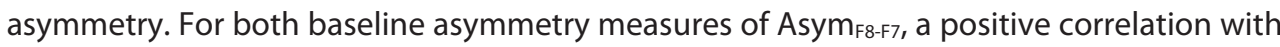
BAS drive (IAF: $r_{60}=.35 ; p=.006$; Standard Alpha: $\left.r_{60}=.32 ; p=.01\right)$ and a negative correlation with BIS (IAF: $r_{60}=-.33 ; p=.01$; Standard Alpha: $r_{67}=-.30 ; p=.02$ ) was found, indicating that more left-sided frontal activity at baseline was associated with more behavioural activation (see Fig. 4.5). The association with FA was specific for the lateral frontal electrodes (F8-F7) since all other correlations were non-significant (all $p s>.10$ ). Bivariate Pearson correlations between BIS/BAS and stress-reactivity FA did not reveal any significant associations for both standard alpha and IAF (all ps > .21). Bivariate Pearson correlations between BIS/BAS and the participants' physiological stress response did not reveal any significant associations (all ps $>.17)$. 


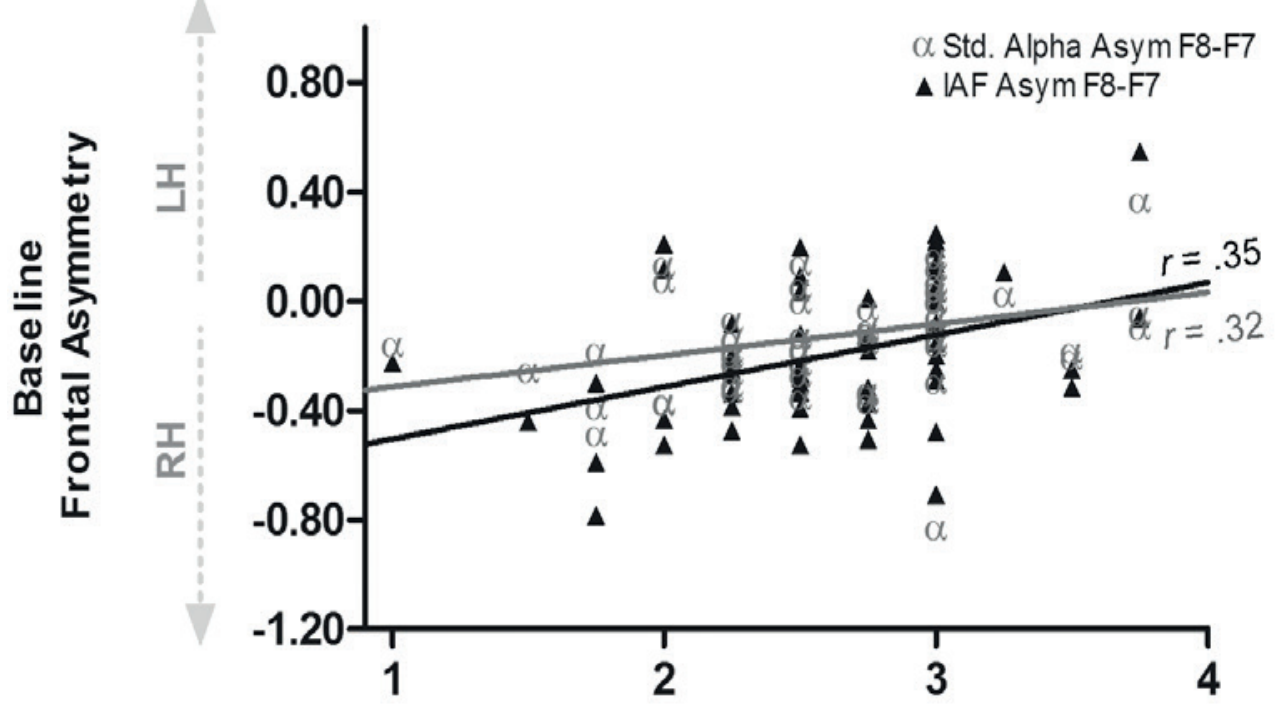

BAS Drive

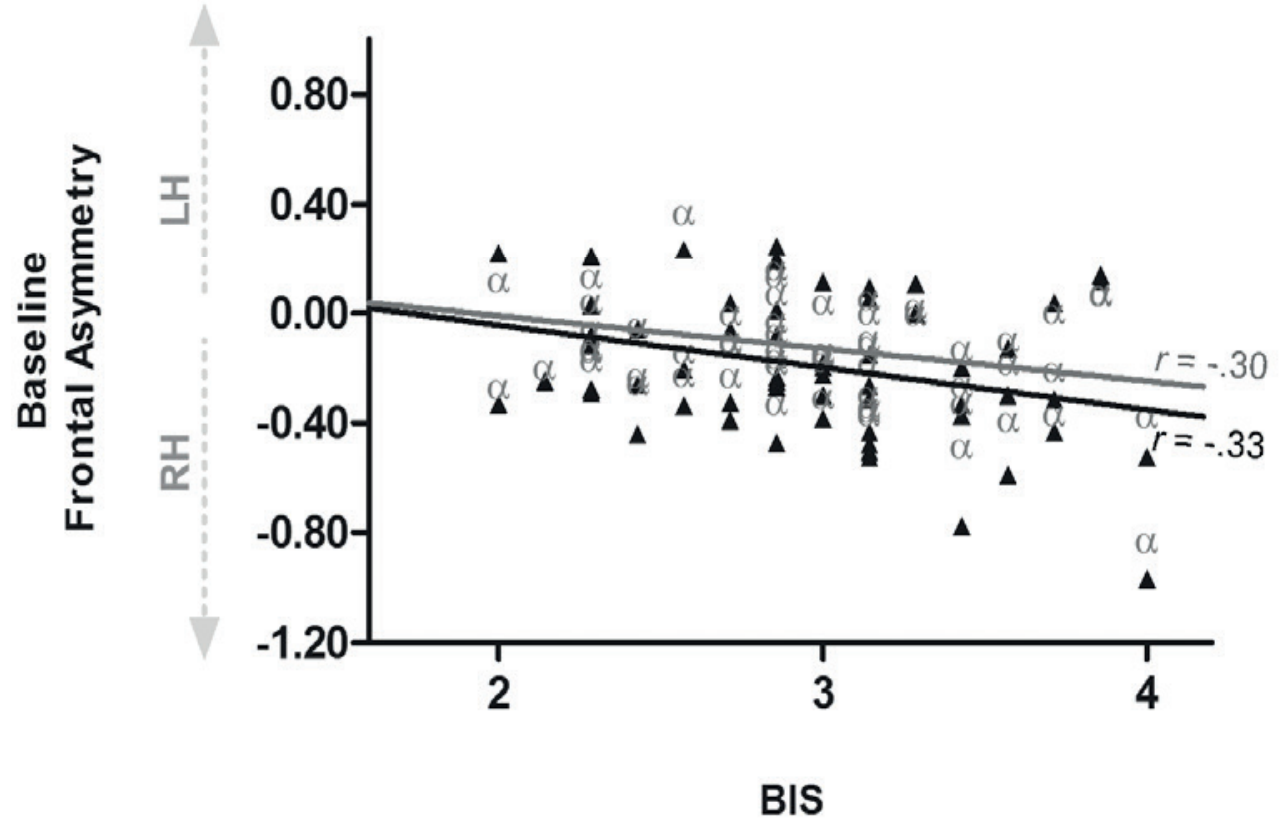

Figure 4.5 Association between frontal asymmetry and behavioural activation. Baseline asymmetry at F8-F7 was positively associated with the BAS drive subscale and negatively with BIS.

\section{Discussion}

The aim of the current study was to investigate the functional role of asymmetric frontal alpha oscillations in stress-induced neuroendocrine responses. Secondly, we explored whether the relationship between FA and individual differences in stress responding are more reliably described if based on IAF instead of the standard $8-13 \mathrm{~Hz}$ alpha band. Resting EEG was measured before and after stress induction by means of the MAST ${ }^{30}$. The MAST, indeed, generated robust increases in cortisol levels. The cortisol responses were moderated by gender, which is in line with the typically larger increases found in men compared to women in response to laboratory stressors ${ }^{31,40}$. We found that higher left hemispheric activity at baseline was associated with lower stress-induced cortisol levels (AUCi), an effect that was specific to the sites F4-F3 and was only significant when IAF was used. Besides, we found higher baseline $\mathrm{FA}_{\mathrm{F} 8-\mathrm{F} 7}$ to correlate with higher BAS and lower BIS scores. Meanwhile, the stress induction did not result in a change in $F A$, which suggests that individual FA might reflect a trait-like characteristic that relates to cortisol reactivity to a stressor.

The finding that IAF based baseline $\mathrm{FA}_{\mathrm{F} 4-\mathrm{F} 3}$ was associated with lower stress-induced cortisol levels (AUCi) is in line with our expectations and agrees well with studies suggesting that left frontal activity is involved in the regulation of hormonal stress responses ${ }^{15,16,19}$. No association was found when FA was based on the standard $8-13 \mathrm{~Hz}$ alpha band, which is in agreement with previous studies investigating the relation between cortisol and FA in the standard alpha band ${ }^{14,21,23}$. The mean of the IAF was not different from the centre of the standard alpha band indicating that the difference in sensitivity and reliability cannot be explained by the use of alpha sub-bands that are, across participants, consistently different from standard alpha. Our results support the idea that the individualized alpha band (IAF) is more reliable than standard alpha in the prediction of individual differences in the processing of stressors.

The current results suggest an association of BAS drive with baseline left lateral frontal activity and an association of BIS with baseline right lateral frontal activity (i.e., Asym $_{F-F-77}$. Studies that have investigated the relation between BIS and frontal lateralisation have yielded divergent findings, with most reporting weak or no association ${ }^{41-44}$ while Sutton and Davidson ${ }^{45}$ also found a negative correlation. Nevertheless, in line with the current findings, all before mentioned studies hypothesized to find a negative correlation between BIS and FA. BIS is a complex motivational concept ${ }^{46}$ that probably is reflected in a 
less robust relation with right-sided FA. In terms of BAS, relative left sided FA was related to more behavioural drive. This result is consistent with previous studies ${ }^{4-49}$ and more general with studies associating frontal asymmetry with overall behavioural activation ${ }^{41,43,45,46}$. A noteworthy observation is that most studies found associations between the behavioural activation system and mid-frontal (F4-F3) locations corresponding to the dorsolateral prefrontal cortex $(\mathrm{dIPFC})^{50}$. Still, associations with lateral FA (F8-F7) have also been demonstrated in previous studies ${ }^{41,44}$, suggesting a more ventrolateral PFC source. Numerous neuroimaging studies have demonstrated opposite affective lateralized processing effects within specific areas of the $\mathrm{PFC}^{51,52}$, which could explain these divergent location findings and indicates the need to measure EEG at a high spatial density and combine EEG and $\mathrm{MMRI}^{53}$.

The finding that FA remained unaffected by stress is at first glance at odds with the study of Lewis and colleagues ${ }^{23}$ and the prior cortisol administration studies of Tops and colleagues ${ }^{21,22}$. Yet, Lewis and colleagues ${ }^{23}$ found different results when applying a region or a single electrode analysis and in line with our results, no effect of examination stress was found for the single electrode analysis of F4-F3. One explanation for the disparity with the studies of Tops and colleagues ${ }^{21,22}$ may be that exogenous cortisol administration activates the HPA-axis at a different level than a psychological stressor, which also increases the release of other hormones such as catecholamines ${ }^{31}$. Moreover, the timing of the EEG measurements could also account for the differential findings, as Tops and colleagues ${ }^{21,22}$ collapsed multiple asymmetry measurements over 30-min and 2-h, respectively, whereas the current study did not. The current finding that stress induction did not result in a change in FA while at the same time individual $\mathrm{FA}_{\mathrm{F} 4 \mathrm{~F} 3}$ predicted the cortisol response, suggests that individual FA reflects individual differences in a trait-like mechanism that moderates the cortisol stress response.

A few limitations of the current study should be noted. First, this study was performed in healthy subjects. Cortical asymmetry scores are expected to reflect more symmetrical activity in healthy subjects than in clinical populations $s^{54,55}$ and therefore statistical results might be dampened. Second, FA was measured before and after the MAST, but not during the MAST. This was done because the MAST consists of mental arithmetic trials, and the induced cognitive effort could confound effects of stress on alpha activity when measured during the MAST. Thus, while we found no change in FA in response to the
MAST, FA may still have transiently changed during stress. Finally, the present study is limited in generalizability by the fact that cortisol but no other measures of the stress response, such as autonomic or subjective, were assessed.

In sum, the present findings suggest that baseline frontal alpha activity reflects a mechanism that has a moderating role in the fight-or-flight response to acute stress. Specifically, relatively left activation appears to be associated with resilience characterized by behavioural activation and neuroendocrine regulation. Thereby, this study contributes to the relatively sparse and inconsistent literature regarding the role of trait characteristics in moderating responses to state manipulation. Our data show that hemispheric asymmetry measures based on frontal alpha frequencies may serve as a better individual difference variable if they are based on individualized alpha frequencies than if they are based on standard alpha $(8-13 \mathrm{~Hz})$. Constructing frontal asymmetry on the individualized alpha band seems a promising avenue to the refinement and extension of our knowledge of the role of asymmetric hemispheric activation as a determinant of individuals' degree of stress resilience or vulnerability. 


\section{References}

1 Coan, J.A., Allen, J.J.B. \& McKnight, P.E. (2006). A capability model of individua differences in frontal EEG asymmetry. Biological Psychology, 72, 198-207.

2 Harmon-Jones, E., Gable, P.A. \& Peterson, C.K. (2010). The role of asymmetric frontal cortical activity in emotion-related phenomena: a review and update. Biological Psychology, 84, 451-462.

3 Heller, W. (1993). Neuropsychological mechanisms of individual differences in emotion, personality, and arousal. Neuropsychology, 7, 476-489.

4 Coan, J.A. \& Allen, J.J.B. (2003). The State and Trait nature of Frontal EEG Asymmetry in Emotion In Kenneth Hughdahl and Richard J. Davidson (Ed.) The asymmetrical brain (pp. 565-616). Cambridge, Massachusetts, London England: The MIT Press.

5 Coan, J.A. \& Allen, J.J.B. (2004). Frontal EEG asymmetry as a moderator and mediator of emotion. Biological Psychology, 67, 7-49.

6 Goodman, R.N., Rietschel, J.C., Lo, L.C. Costanzo, M.E. \& Hatfield, B.D. (2013). Stress, emotion regulation and cognitive performance: The predictive contributions of trait and state relative frontal EEG alpha asymmetry. International Journal of Psychophysiology, 87, 115-123.

7 Perez-Edgar, K., Kujawa, A., Nelson, S. K. Cole, C. \& Zapp, D.J. (2013). The relation between electroencephalogram asymmetry and attention biases to threat at baseline and under stress. Brain and Cognition, 82, 337-343.

8 Papousek, I., Reiser, E.M., Weber, B. Freudenthaler, H.H. \& Schulter, G. (2012). Frontal brain asymmetry and affective flexibility in an emotional contagion paradigm. Psychophysiology, 49, 489-498.

9 Jackson D.C etal. (2003). Now you feel it, now you don't: Frontal brain electrical asymmetry and individual differences in emotion regulation. Psychological Science, 14, 612-617.

10 Tomarken, A.J., Davidson, R.J., Wheeler, R.E. \& Doss, R.C. (1992). Individual Differences in Anterior Brain Asymmetry and Fundamental Dimensions of Emotion. Journal of Personality and Social Psychology, 62, 676-687.

11 Kalin, N.H., Larson, C., Shelton, S.E. \& Davidson, R.J. (1998). Asymmetric frontal brain activity, cortisol, and behaviour associated with fearful temperament in rhesus monkeys. Behavioural Neuroscience, 112, 286-292.

12 Rilling, J.K. et al. (2001). Neural correlates of maternal separation in Rhesus monkeys. Biological Psychiatry, 49, 146157.

13 Buss, K.A. et al. (2003). Right frontal brain activity, cortisol, and withdrawal behaviour in 6-month-old infants. Behavioural Neuroscience, 117, 11-20.

14 Hewig, J. et al. (2008). Associations of the cortisol awakening response (CAR) with cortical activation asymmetry during the course of an exam stress period. Psychoneuroendocrinology, 33, 83-91.

15 Baeken, C. et al. (2014). One left dorsolateral prefrontal cortical HF-rTMS session attenuates HPA-system sensitivity to critical feedback in healthy females. Neuropsychologia, 57, 112-121.

16 Davidson, R.J. (2000). Affective style, psychopathology, and resilience: Brain mechanisms and plasticity. American Psychologist, 55, 1196-1214.

17 Lueken, U. et al. (2009). Altered tonic and phasic cortisol secretion following unilateral stroke. Psychoneuroendocrinology, 34, 402-412.

18 Sullivan, R.M. (2004). Hemispheric asymmetry in stress processing in rat prefrontal cortex and the role of mesocortical dopamine. Stress-the International Journal on the Biology of Stress, 7, 131-143.

19 Sullivan, R.M. \& Gratton, A. (2002). Prefrontal cortical regulation of hypothalamic-pituitary-adrenal function in the rat and implications for psychopathology: side matters. Psychoneuroendocrinology, 27, 99-114.

20 Wittling, W. \& Pfluger, M. (1990). Neuroendocrine hemisphere asymmetries: salivary cortisol secretion during lateralized viewing of emotionrelated and neutral films. Brain and Cognition, 14, 243-265.

21 Tops, M. et al. (2005). Acute cortisol administration modulates EEG alpha asymmetry in volunteers: relevance to depression. Biological Psychology, 69, 181-193.

22 Tops, M., van Peer, J.M., Wester, A.E, Wijers, A.A. \& Korf, J. (2006). Statedependent regulation of cortical activity by cortisol: An EEG study. Neuroscience Letters, 404, 39-43.

23 Lewis, R.S., Weekes, N.Y. \& Wang, T. H. (2007). The effect of a naturalistic stresso on frontal EEG asymmetry, stress, and health. Biological Psychology, 75, 239-247.

24 Bazanova, O.M. \& Vernon, D. (2014). Interpreting EEG alpha activity. Neuroscience and Biobehavioural Reviews, 44C, 94-110.

25 Doppelmayr, M., Klimesch, W., Pachinger T. \& Ripper, B. (1998). Individual differences in brain dynamics: important implications for the calculation of eventrelated band power. Biological Cybernetics, 79, 49-57.

26 Gasser, T., Bacher, P. \& Steinberg, H. (1985). Test-retest reliability of spectral parameters of the EEG. Electroencephalography and Clinical Neurophysiology, 60 312-319.
27 Tomarken, A.J., Davidson, R.J., Wheeler, R.E. \& Kinney, L. (1992). Psychometric properties of resting anterior eeg asymmetry - temporal stability and internal consistency. Psychophysiology, 29, 576-592.

28 Klimesch, W., Schimke, H. \& Pfurtscheller, G. (1993). Alpha frequency, cognitive load and memory performance. Brain Topography, 5, 241-251.

29 Klimesch, W. (1999). EEG alpha and thet oscillations reflect cognitive and memory performance: a review and analysis. Brain Research Reviews, 29, 169-195.

30 Smeets, T. et al. (2012). Introducing the Maastricht Acute Stress Test (MAST): A quick and non-invasive approach to elicit robust autonomic and glucocorticoid stress responses. Psychoneuroendocrinology, 37, 1998-2008.

31 Kudielka, B.M., Hellhammer, D.H. \& Wust S. (2009). Why do we respond so differently? Reviewing determinants of human salivary cortisol responses to challenge. Psychoneuroendocrinology, 34, 2-18.

32 Velo, J.R., Stewart, J. L., Hasler, B.P. Towers, D.N. \& Allen, J.J.B. (2012). Should it matter when we record? Time of year and time of day as factors influencing frontal EEG asymmetry. Biological Psychology, 91, 283-291.

33 Kudielka, B.M., Hellhammer, D.H. \& Kirschbaum, C. (2007). Ten Years of Research with the Trier Social Stress Test-Revisited. In E. Harmon-Jones \& P. Winkielman (Eds.), Social neuroscience: Integrating biological and psychological explanations of social behaviour (pp. 5683). New York, NY, US: The Guilford Press.

34 Pruessner, J.C., Kirschbaum, C., Meinlschmid, G. \& Hellhammer, D.H. (2003). Two formulas for computation of the area under the curve represent measures of total hormone concentration versus time-dependent 
change. Psychoneuroendocrinology, 28, 916-931.

35 Carver, C.S. \& White, T.L. (1994) Behavioural-Inhibition, Behavioural Activation, and Affective Responses to Impending Reward and Punishment - the Bis Bas Scales. Journal of Personality and Social Psychology, 67, 319-333.

36 Meyer, T. et al. (2014). Frontal EEG asymmetry as predictor of physiological responses to aversive memories Psychophysiology, 51, 853-865.

37 Allen, J.J.B., Coan, J.A. \& Nazarian, M. (2004). Issues and assumptions on the road from raw signals to metrics of frontal EEG asymmetry in emotion. Biological Psychology, 67, 183-218.

38 Gratton, G., Coles, M.G. \& Donchin, E. (1983). A new method for off-line removal of ocular artifact. Electroencephalography and Clinical Neurophysiology, 55, 468-484.

39 Pfurtscheller, G., Stancak, A. \& Neuper, C. (1996). Event-related synchronization (ERS) in the alpha band - An electrophysiological correlate of cortica idling: A review. International Journal of Psycho-physiology, 24, 39-46.

40 Meyer, T., Smeets, T., Giesbrecht, T. Quaedflieg, C.W.E.M. \& Merckelbach, H. (2013). Acute stress differentially affects spatial configuration learning in high and low cortisol-responding healthy adults. European Journal of Psycho-traumatology, 4.

41 Coan, J.A. \& Allen, J.J.B. (2003). Frontal EEG asymmetry and the behavioura activation and inhibition systems. Psychophysiology, 40, 106-114.

42 De Pascalis, V., Cozzuto, G., Caprara, G.V. \& Alessandri, G. (2013). Relations among EEG-alpha asymmetry, BIS/BAS, and dispositional optimism. Biological Psychology, 94, 198-209.

43 Harmon-Jones, E. \& Allen, J.J.B. (1997). Behavioural activation sensitivity and resting frontal EEG asymmetry: Covariation of putative indicators related to risk for mood disorders. Journal of Abnormal Psychology, 106, 159-163.

44 Hewig, J., Hagemann, D., Seifert, J. Naumann, E. \& Bartussek, D. (2006). The relation of cortical activity and BIS/BAS on the trait level. Biological Psychology, 71, 42-53.

45 Sutton, S.K. \& Davidson, R.J. (1997) Prefrontal brain asymmetry: A biological substrate of the behavioural approach and inhibition systems. Psychological Science, 8, 204-210.

46 Amodio, D.M., Master, S.L., Yee, C.M. \& Taylor, S.E. (2008). Neurocognitive components of the behavioural inhibition and activation systems: implications for theories of selfregulation. Psychophysiology, 45, 11-19.

47 Berkman, E.T. \& Lieberman, M.D. (2010) Approaching the bad and avoiding the good: lateral prefrontal cortical asymmetry distinguishes between action and valence. Journal of Cognitive Neuroscience, 22, 1970-1979.

48 De Pascalis, V., Varriale, V. \& D'Antuono, L. (2010). Event-related components of the punishment and reward sensitivity. Clinical Neurophysiology, 121, 60-76.

49 Tops, M. \& Boksem, M.A. (2010). Absorbed in the task: Personality measures predic engagement during task performance as tracked by error negativity and asymmetrical frontal activity. Cognitive, Affective \& Behavioural Neuroscience, 10 441-453.

50 Herwig, U., Satrapi, P. \& Schonfeldt Lecuona, C. (2003). Using the international 10-20 EEG system for positioning of transcranial magnetic stimulation. Brain Topography, 16, 95-99.

51 Miller, G.A., Crocker, L.D., Spielberg, J. M., Infantolino, Z.P. \& Heller, W. (2013). Issues in localization of brain function: The case of lateralized frontal cortex in cognition, emotion, and psycho-pathology Frontiers in Integrative Neuroscience, 7, 2.

52 Wager, T.D., Phan, K.L., Liberzon, I. \& Taylor, S.F. (2003). Valence, gender, and lateralization of functional brain anatomy in emotion: a meta-analysis of findings from neuroimaging. Neuroimage, 19, 513-531.

53 Davidson, R.J. (2004). What does the prefrontal cortex "do" in affect: perspectives on frontal EEG asymmetry research. Biological Psychology, 67, 219233.

54 Gordon, E., Palmer, D.M. \& Cooper, N. (2010). EEG alpha asymmetry in schizophrenia, depression, PTSD, panic disorder, ADHD and conduct disorder. Clinical EEG and Neuroscience, 41, 178183.

55 Quinn, C.R., Rennie, C.J., Harris, A.W. \& Kemp, A.H. (2014). The impact of melancholia versus non-melancholia on resting-state, EEG alpha asymmetry: electrophysiological evidence for depression heterogeneity. Psychiatry Research, 215, 614-617. 


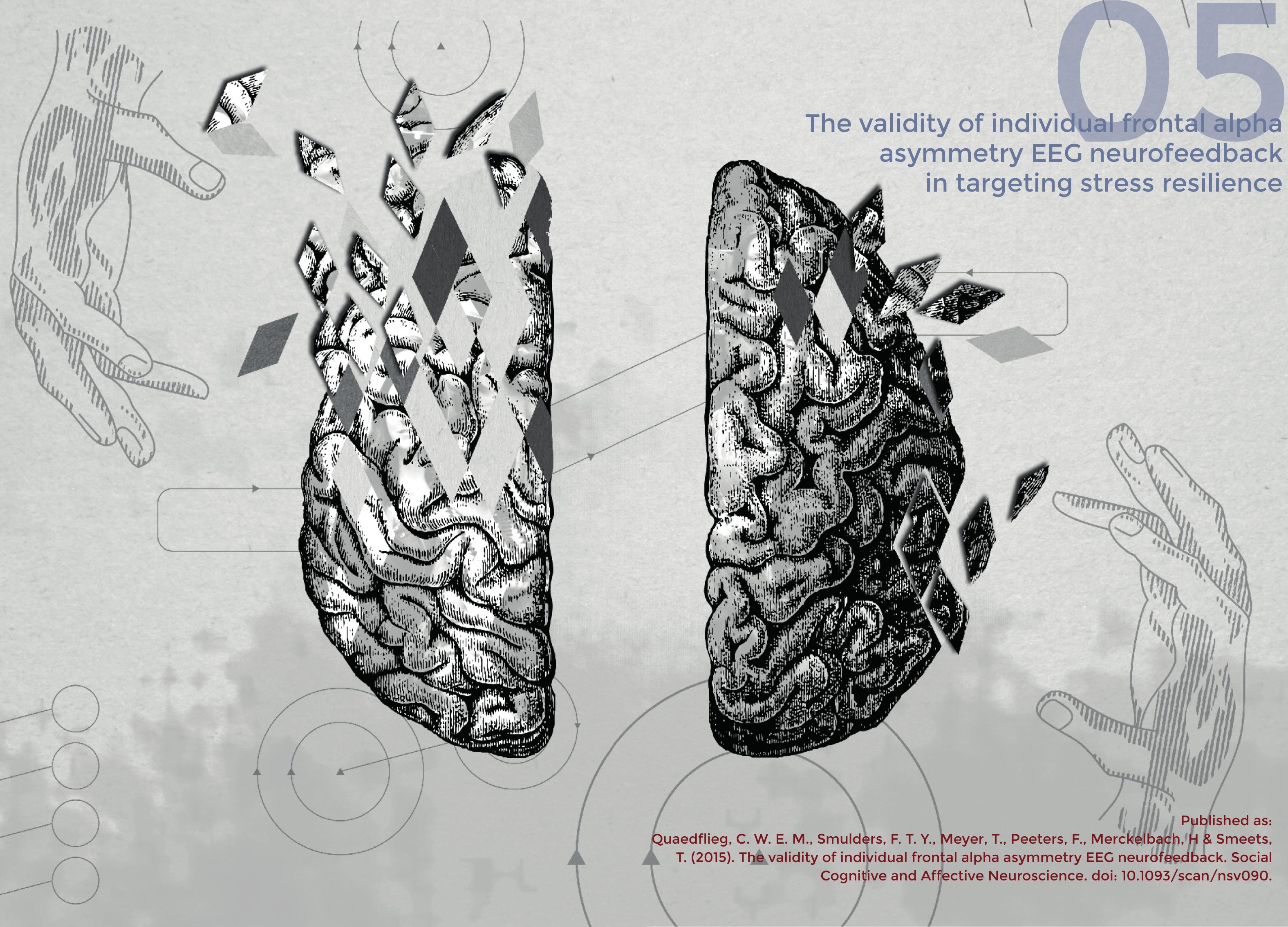




\section{Abstract}

Frontal asymmetry in alpha oscillations is assumed to be associated with psychopathology and individual differences in emotional responding. Brain-activity-based feedback is a promising tool for the modulation of cortical activity. Here, we validated a neurofeedback protocol designed to change relative frontal asymmetry based on individual alpha peak frequencies (IAF), including real-time average referencing and eye-correction. Participants $(N=60)$ were randomly assigned to a right, left, or placebo neurofeedback group. Results show a difference in trainability between groups, with a linear change in frontal alpha asymmetry over time for the right neurofeedback group during rest. Moreover, the asymmetry changes in the right group were frequency and location specific, even though trainability did not persist at one week and one month follow-ups. On the behavioural level, subjective stress on the second test day was reduced in the left and placebo neurofeedback groups, but not in the right neurofeedback group. We found individual differences in trainability that were dependent on training group, with participants in the right neurofeedback group being more likely to change their frontal asymmetry in the desired direction. Individual differences in trainability were also reflected in the ability to change frontal asymmetry during the feedback.

Keywords: frontal asymmetry, randomized placebo control design, trainability, specificity, interpretability 
Frontal asymmetry has been studied extensively in individual differences research on emotional and motivational processes. It refers to the average difference in brain activity between the left and right frontal areas, measured as hemispheric differences in alpha power in electroencephalography (EEG) recordings across several minutes'. Activity in the left-frontal hemisphere has been linked to an approach system that is activated when an individual is moving towards goals or experiences positive emotions. Conversely, a right lateralised withdrawal system is involved in negative affect or the motivation to move away from potentially dangerous situations or stimuli ${ }^{2-4}$.

Frontal asymmetry has important implications for mental health and stress adaptation ${ }^{5,6}$. For instance, greater left-sided frontal activity at rest was found to predict greater emotional flexibility ${ }^{7}$, better emotion regulation ${ }^{8}$, more positive and decreased negative affect ${ }^{4}$, as well as a smaller task-induced cortisol increase ${ }^{9}$. Moreover, extreme right frontal asymmetry has been associated with affective disorders such as depression ${ }^{10}$ and social anxiety disorder (SAD) ${ }^{11}$. In addition, increasing left-sided activity or decreasing rightsided activity with repetitive transcranial magnetic stimulation (rTMS) resulted in improvements in depression ${ }^{12}$ and anxiety ${ }^{13}$ symptoms.

EEG neurofeedback is another method used to modulate cortical activity. The individual is given feedback on his or her current brain activity, based on real-time analysis of EEG signals. In order to induce a specific pattern of brain activity, the individual receives 'rewarding' feedback whenever the EEG changes in the preferred direction. Using this positive reinforcement, the desired brain activity is learned through operant conditioning ${ }^{1,14}$. There has been a recent surge in the use of neurofeedback for the treatment of several kinds of disorders and preliminary studies suggest its clinical efficacy (see for example $A D H D^{15}$ but see 16 , Depression ${ }^{17,18}$ ). Even though neurofeedback has often been applied in clinical research, the validation of neurofeedback as a treatment protocol lags behind ${ }^{19,20}$. To assess the development of self-regulation, three criteria have been proposed $^{21}$. First, the trained frequency needs to change significantly (i.e., trainability). If trainability is successful, the specificity in the trained frequency band and location (i.e., independence) as well as the stability over time has to be assessed. Finally, the behavioural effects of the training need to be determined (i.e., interpretability).

EEG neurofeedback has often been used in attempts at changing frontal asymmetry (i.e., F4-F3). Allen and colleagues ${ }^{22}$ investigated the effect of five frontal alpha asymmetry neurofeedback sessions on participants' emotional responses to movies. Training healthy participants to display relative right-sided frontal activity was associated with less approach responses to happy movies. However, they did not investigate the independence or stability of the training. Independence of location of frontal alpha asymmetry neurofeedback training was assessed by Harmon-Jones and colleagues ${ }^{23}$, who trained participants for two days, and found a specific difference in the change in alpha asymmetry on frontal but not on parietal electrodes for the relative left frontal asymmetry group. Recently, Peeters and colleagues $^{24}$ investigated the possibility of changing frontal asymmetry in a single neurofeedback session. They reported that it is feasible to change frontal asymmetry in both directions in healthy participants, and that this change is specific in terms of location. All in all, the effectiveness of neurofeedback trainings to change relative frontal asymmetry and mood state is in dire need of a more thorough empirical validation.

In light of these considerations, the present study aimed to further validate and explore the potential of frontal alpha asymmetry neurofeedback. In particular, we compared three frontal asymmetry protocols that were developed to increase relative right-sided frontal alpha asymmetry, to increase relative left-sided frontal alpha asymmetry, or to yield no effects on frontal alpha asymmetry (i.e., the placebo control group). Extending prior studies that examined the effect of neurofeedback on frontal asymmetry, we determined frontal asymmetry for each participant based on individual alpha peak frequency (IAF). The main advantage of this approach is that it controls for the large individual differences in alpha frequency ${ }^{25,26}$ that may impair the trainability of frontal asymmetry based on conventional frequency bands. We hypothesised that following six days of neurofeedback, participants would display the intended change in frontal asymmetry along with a change in current mood and task-induced subjective and neuroendocrine stress responses. Specifically, participants trained to shift relative frontal alpha power towards the right hemisphere were expected to show decreased negative affect and decreased subjective and neuroendocrine stress responses over time. The opposite pattern of findings was expected for the left group. No changes over time were hypothesized for the placebo group. Additionally, we explored whether gender differences in the effectiveness of neurofeedback training exist. Furthermore, for both the left and right group, we expected that the frontal alpha asymmetry training would result in changes specifically in the (individual) alpha band and solely at frontal locations. In addition, we explored whether the asymmetry changes 
would persist up to one month following the final neurofeedback session. Finally, based on previous studies demonstrating large individual differences in the ability to learn how to regulate cortical activity ${ }^{21,27-29}$, we classified and compared participants who responded well to the training to those who did not or did so to a lesser degree.

\section{Methods}

\section{Participants}

The present experiment was part of a larger study that investigated the effect of neurofeedback on resilience ${ }^{9}$. Right-handed healthy men $(n=30)$ and women $(n=30)$ undergraduates (mean age $=20.96 S D=2.82$; range: 18-31 years) were recruited via advertisements at Maastricht University. Participants were screened for eligibility using the following exclusion criteria: history of psychiatric, neurologic, cardiovascular or neuroendocrine diseases, heavy smoking (i.e., more than 15 cigarettes/day), medication use known to affect the autonomic nervous system (ANS) or hypothalamic-pituitary-adrenal (HPA) axis, drug use, and body mass index (BMI) outside the normal range (i.e., $18-30 \mathrm{~kg} / \mathrm{m}^{2}$ ). For women, the use of oral contraceptives served as an inclusion criterion to reduce variability in cortisol responses resulting from hormonal alterations ${ }^{30}$. Test protocols were approved by the standing ethics committee of the Faculty of Psychology and Neuroscience, Maastricht University. All participants provided written informed consent and were given a minor incentive (course credits or money) in return for their participation.

\section{EEG data acquisition}

The electroencephalogram (EEG) was recorded using a BrainAmp amplifier and BrainVision Recorder software (BrainProducts, Germany) from $23 \mathrm{Ag} / \mathrm{AgCl}$ electrodes (F7, F3, Fz, F4, F8, FC3, FC4, T7, T8, C3, Cz, C4, CP3, CPz, CP4, P7, P3, Pz, P4, P8, O1, Oz, O2) positioned in an elastic cap according to the international 10-20 system, referenced to the left mastoid (A1). Signals at A2 were also recorded for re-referencing to computerized linked mastoids. Signals were sampled continuously at $100 \mathrm{~Hz}$ and band-pass filtered $(0.01-30 \mathrm{~Hz})$. An electrode at AFz served as ground. Two electrodes at the outer canthi of both eyes recorded horizontal eye movements and two electrodes above and below the left eye recorded vertical eye movements. Scalp-electrode impedance was kept below $5 \mathrm{k} \Omega$ to ensure high-quality EEG recordings and homologous scalp electrodes were within $1 \mathrm{k} \Omega$ of each other. Participants were shown the raw recording signals to demonstrate common artefacts that occur due to body and eye movements.

Before the start of the first neurofeedback session, the individual alpha peak frequency (IAF) was determined as the dominant frequency rhythm between 5 and $15 \mathrm{~Hz}$ at the posterior electrode $(\mathrm{Pz})$ on 3-min of resting eyes closed data ${ }^{25,31}$. The IAF bandwidth was defined as the IAF $\pm 0.20 \times I A F$. The same bandwidth was used for all six training sessions since IAF has been shown to be stable over time ${ }^{32}$. The frontal alpha asymmetry scores were determined in the IAF band.

Resting frontal asymmetry was measured twice during 4-min, at the beginning and the end of the test or training session, whereby participants focused on a black fixation cross on a grey background on the computer monitor.

\section{Procedure}

An overview of the experimental procedure is displayed in Fig. 5.1. The experiment consisted of a baseline test day followed by six neurofeedback sessions and two follow-up test days, one week and one month later. The six neurofeedback sessions were distributed over the course of two weeks. All testing took place between 12:30 and 18:00-h to avoid morning fluctuations in the circadian rhythm of cortisol and time-of-day effects on frontal asymmetry ${ }^{33}$. Participants were instructed to refrain from eating, exercising extensively, or drinking anything but non-sparkling water for 2-h prior to the experimental session. Upon arrival in the laboratory, a bogus saliva sample was taken to increase participants' honesty in disclosing non-adherence to these instruction $\mathrm{s}^{34}$. Participants were seated in front of a 22 inch widescreen monitor (Philips, The Netherlands) at approximately $56 \mathrm{~cm}$ viewing distance. 


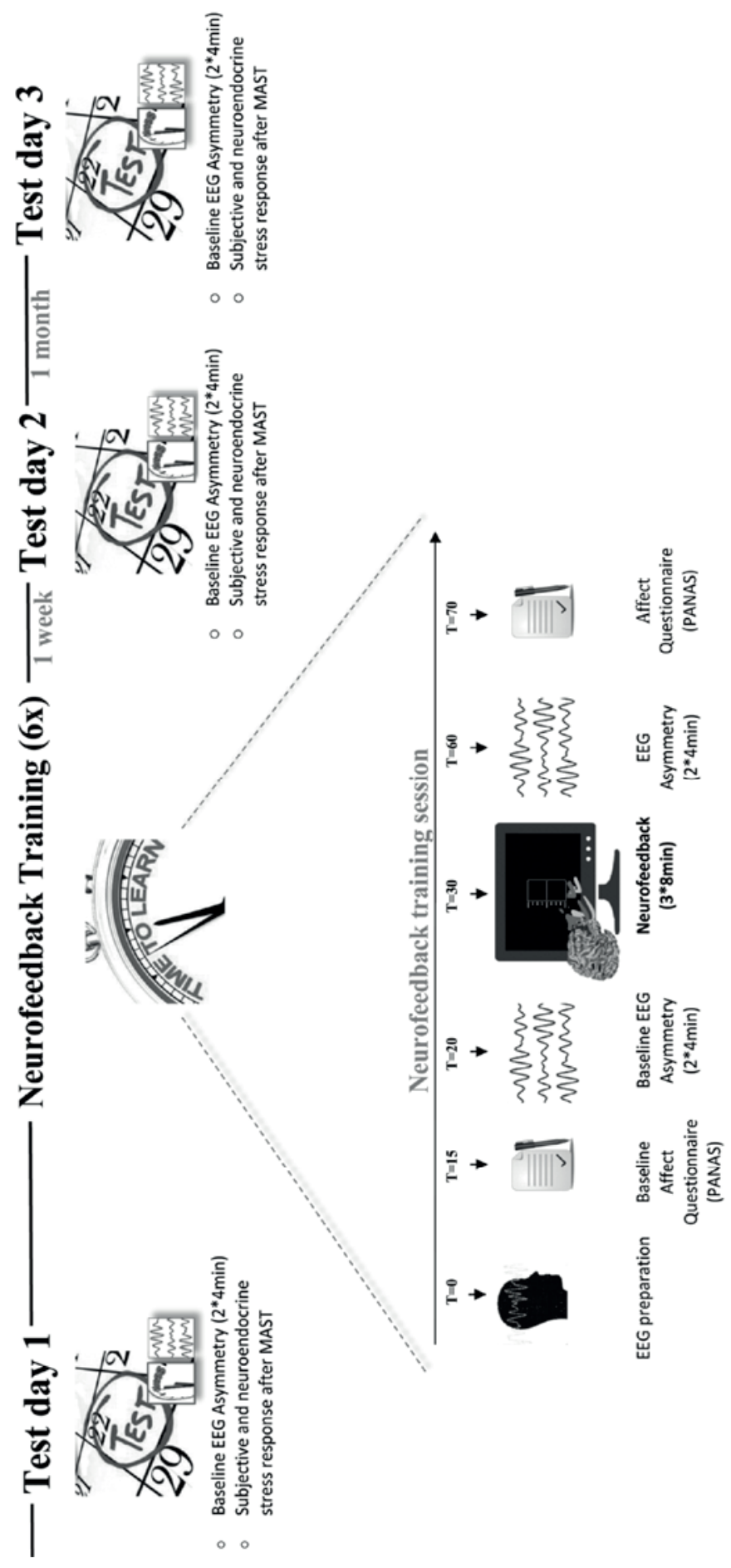

Figure 5.1 Overview of the study design and neurofeedback training sessions.

\section{Test days}

Each test day consisted of a baseline asymmetry measurement after which stress was induced using the Maastricht Acute Stress Test (MAST) ${ }^{35}$. The MAST consists of a 5-min preparation phase in which the task is explained and a 10-min acute stress phase that includes alternating trials of immersing their hand into ice water $\left(2^{\circ} \mathrm{C}\right)$ and counting backwards in steps of 17 starting at 2043 along with social-evaluative pressure (i.e., negative feedback and videotaping). Neuroendocrine and subjective stress responses were measured with synthetic Salivettes (Sarstedt ${ }^{\circ}$, Etten-Leur, The Netherlands) and $100 \mathrm{~mm}$ Visual Analogue scales (VAS). Participants provided saliva samples 20-min after arrival in the lab $\left(t_{\text {base }}\right), 5$-min before $\left(t_{\text {pre-stress }}\right)$ the MAST and five times afterwards $\left(t_{+0}, t_{+10,}, t_{+30}, t_{+40,} t_{+55 \min }\right.$ with reference to the end of the stressor). The Area Under the Curve with respect to increase (AUCi) from the pre-stress sample was calculated as a single measure of the total cortisol concentration in response to the MAST for each participant individually ${ }^{36}$.

Samples were stored at $-20^{\circ} \mathrm{C}$ until cortisol levels were determined by a commercially available luminescence immune assay kit (IBL, Hamburg, Germany). Mean intra- and interassay coefficients of variation are typically less than $5 \%$, and the lower and upper detection limits were $0.015 \mathrm{mg} / \mathrm{dl}(0.41 \mathrm{nmol} / \mathrm{l})$ and $4.0 \mathrm{mg} / \mathrm{dl}(110.4 \mathrm{nmol} / \mathrm{l})$, respectively.

\section{Training days}

Each neurofeedback session consisted of a baseline asymmetry measurement, three neurofeedback blocks, and a post-neurofeedback asymmetry measurement (see Fig. 5.1). Current negative affect was assessed using the state version of the Positive and Negative Affect Schedule (PANAS) ${ }^{37}$. The PANAS consists of 20 items divided in two subscales that quantify current positive affect (PA) and negative affect (NA) using 5-point scales (anchors: $1=$ very slightly or not at all; $5=$ extremely). Higher scores on the NA scale are indicative of higher levels of experienced negative affect.

\section{EEG neurofeedback training}

A double-blind placebo controlled design was applied in which subjects were randomly assigned to one of three frontal alpha asymmetry neurofeedback groups. The power of alpha oscillations is inversely related to brain activity ${ }^{38-41}$. Thus, a brain that is said to be 
relative asymmetric to the right has an alpha predominance in the left hemisphere. Participants in the left neurofeedback group received positive feedback if they increased relative right alpha power. Participants in the right neurofeedback group received positive feedback if they increased relative left alpha power. Participants in the placebo group were not fed back their own current asymmetry. Instead, the feedback received by the placebo group was based on neurofeedback training scores extracted from a pilot study with ten participants. The placebo feedback included randomized data from three left and three right neurofeedback sessions and was unique for each participant.

Each neurofeedback session consisted of three neurofeedback training blocks of 8min. Real-time calculations were done by a filter written for BrainVision Recview (Brain Products, Germany) and included re-referencing to an average A1 and A2 reference, eye blink correction, epoching, transformation to the frequency domain, and asymmetry calculation. Online eye blink correction and re-referencing were performed since eye movement artefacts influence the EEG activity especially at the frontal sites and in the alpha band, and because computerized linked mastoids (i.e., average of $A 1+A 2$ ) reference has a somewhat superior signal-to-noise ratio compared to $C z$ as a reference ${ }^{2,42}$. Re-referencing and eye correction were performed with a linear derivation. The eye correction coefficients were determined in every neurofeedback training session using linear regression ${ }^{43}$ implemented in a plugin written for EEGLAB ${ }^{44}$. To compute power density values, corrected data were divided in 2-s epochs with 75\% overlap and then transformed to the frequency domain using a fast-Fourier transformation (FFT; 100\% Hanning window). Asymmetry scores were calculated every 0.5 -s in the individual alpha frequency band as log-transformed alpha-power density values, In (F4) - In (F3). Positive alpha asymmetry scores indicate greater relative left than right frontal activity; negative alpha asymmetry scores indicate greater relative right than left frontal activity. In order to provide smooth feedback to the participant, the 10 last asymmetry values were included in a linear weighted (i.e., oldest data given a weight of 0.1 and newest a weight of 1) moving average.

The feedback stimulus was presented visually via a PC using Presentation (Neurobehavioural Systems), in the form of a boxplot-like meter. The start position of the line of the meter was always in the middle and represented the baseline asymmetry of the training day. The position of the line provided real-time feedback with regard to the baseline asymmetry and was above the middle if the current relative asymmetry was a shift in the desired direction compared to the baseline of the day. Moreover, the colour of the line provided feedback with regard to the asymmetry measured 0.5 -s before and became green if the current asymmetry was a shift in the desired direction compared to the previous measurement. A numerical value that represented overall training performance, and which was continuously upgraded during the 8-min neurofeedback blocks, was displayed below the meter ${ }^{24}$. The increase of the score represented the size of the shift in the asymmetry in the desired direction. It was explained to the participants that the line represented their brain activity at that moment and that this line would turn green if they would have the desired activity. They were instructed to try to earn as many points as they could by keeping the line of the meter green. It was made clear to participants that the total score was unrelated to the compensation that the participants would be receiving. Analyses showed that the average score that was attained, was the same for the left and right neurofeedback training group!

\section{EEG offline analysis and statistical analysis}

Offline analyses were performed with Vision Analyzer 2.0 (Brain Products, Germany). The offline derivation of frontal asymmetry scores was consistent with previous studies ${ }^{45,46}$ and almost identical to the real-time data steps (see EEG neurofeedback training). One step was added, namely epochs containing EEG changes exceeding $\pm 75 \mu \mathrm{V}$ were automatically omitted from averages. On average, 735 epochs of the rest measurements and 701 epochs of the neurofeedback blocks was artefact free. Data from three participants were excluded because less than $30 \%$ of artefact-free epochs were retained and data of one other participant were lost due to recording software failure. Thus, the final sample for the EEG analysis consisted of 56 participants (i.e., right group: 20, left group: 18, placebo: 18). Moreover, the subjective stress data of two participants were missing. The final sample for the behavioural analysis consisted of 54 participants (i.e., right group: 19, left group: 17, placebo: 18)

Statistical analyses were performed on $z$-transformed (i.e., using the mean of rest asymmetry on test day 1 and training 1 and its $S D$ ) frontal asymmetry scores with repeated measures ANOVAs reporting linear contrasts ${ }^{47}$. Follow-up simple effects analyses were

Linear contrasts with training ( 6 levels) and group (left, right) revealed a non-significant interaction effect (training $\times$ group $F_{(1,3)}=0.93, p=.34$ ). 
performed using one-way ANOVAs. A $p$-value less than 0.05 was considered statistically significant and a $p$-value between 0.05 and 0.10 as a statistical trend towards significance. If analyses yielded significant or trend-level findings, ANOVAs were supplemented with Partial Eta Squared $\left(\eta^{2}{ }_{p}\right)$ values as a measure of effect size $\left(\eta^{2}{ }_{p}\right.$ of 0.01 indicate small effects, $\eta^{2}{ }_{p}$ of 0.06 medium effects, and $\eta^{2}{ }_{p}$ of 0.14 large effects $)^{48}$.

\section{Results}

Trainability was assessed by investigating the effect of the neurofeedback protocol on relative frontal asymmetry in the alpha band during the neurofeedback blocks and at rest over the six training sessions. If trainability was successful, the specificity (i.e., frequency and location) and stability as well as the effect on the behavioural level of the neurofeedback training were assessed. Finally, individual differences in self-regulation were evaluated.

\section{The effectiveness of frontal alpha asymmetry neurofeedback}

\section{Trainability}

Fig. 5.2 displays the time course of change in relative frontal alpha asymmetry (F4-F3) during the neurofeedback blocks over 6 neurofeedback training sessions. Trainability, defined as a change in alpha-band frontal asymmetry over training days was assessed first. The linear contrasts with training $(1,2,3,4,5,6)$ and block (block 1, block 2, block 3 ) as linear-subject variables and group (left, right, control) and gender (men, women) as between-subject variables, revealed that training group did not differentially change the relative frontal asymmetry measured over the training days during the feedback (training $\times$ group interaction: $\left.F_{(2,50)}=0.32, p=.72\right)$. Moreover, training had also no effect on frontal asymmetry within sessions measured during the feedback (group $\times$ block interaction: $F_{(2,50)}=1.35, p$ $=.27$; group main: $\left.F_{(2,50)}=0.49, p=.62\right)$ and was not different between men and women (training $\times$ group $\times$ gender interaction: $F_{(2,50)}=0.48, p=.62$ ).

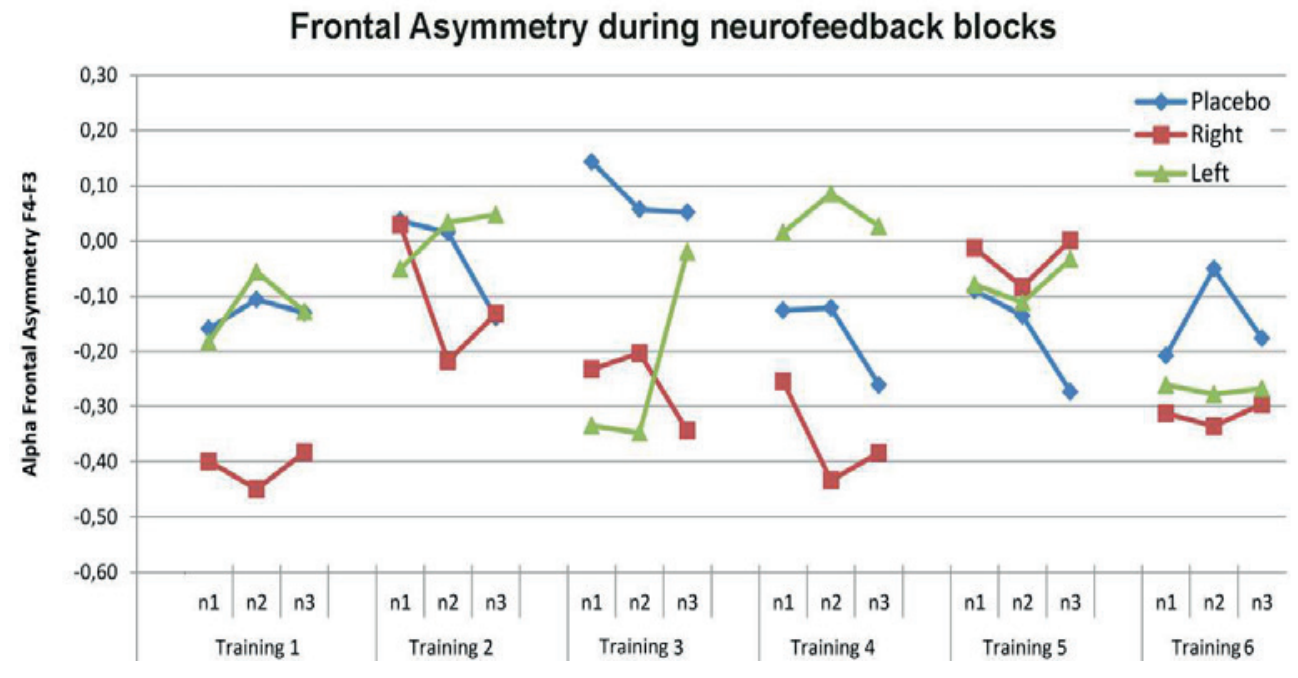

Figure 5.2 Frontal alpha asymmetry (F4-F3) during the neurofeedback blocks over 6 neurofeedback training sessions. Training group did not differentially change the relative frontal asymmetry measured during feedback between day 1 and 6 . Positive alpha asymmetry scores indicate greater relative left than right frontal activity, while negative alpha asymmetry scores indicate greater relative right than left frontal activity. Each neurofeedback session consisted of three neurofeedback blocks. Abbreviations: n1-3= neurofeedback training blocks.

For the frontal asymmetry during rest, the linear contrasts with training $(1,2,3,4,5$, 6) and measurement (pre, post) as within-subject variables and group (left, right, control) and gender (men, women) as between-subject variables revealed a training main effect $\left(F_{(1,50)}=4.14, p=.05 \eta^{2}{ }_{p}=.08\right)$ and a trend-level training $\times$ group interaction $\left(F_{(2,50)}=2.86, p\right.$ $=.07 ; \eta_{\mathrm{p}}^{2}=.10$ ), without a gender difference (training $\times$ group $\times$ gender interaction: $F_{(2,50)}=$ $0.52, p=.60$ ) or measurement (pre-post) effect (measurement main effect $F_{(1,50)}=0.24, p=.63$; training $\times$ group $\times$ measurement interaction: $\left.F_{(2,50)}=1.83, p=.18\right)$. Thus, follow-up analyses were performed across pre and post asymmetry measurements and across gender. The neurofeedback training changed the frontal asymmetry for the right group; the frontal asymmetry became relatively more right sided over training days (training main effect $F_{(1,19)}$ $\left.=10,69 p=.004 ; \eta^{2}=.36\right)$. However, for the left and control group there was no significant main effect of training (left: $F_{(1,17)}=0.65, p=.43$; control: $F_{(1,17)}=0.23, p=.64$ ). Fig. 5.3 displays the time course of change in relative frontal alpha asymmetry (F4-F3) during the rest measurements before and after the neurofeedback blocks over six neurofeedback training sessions per group. 


\section{Frontal Asymmetry during rest}

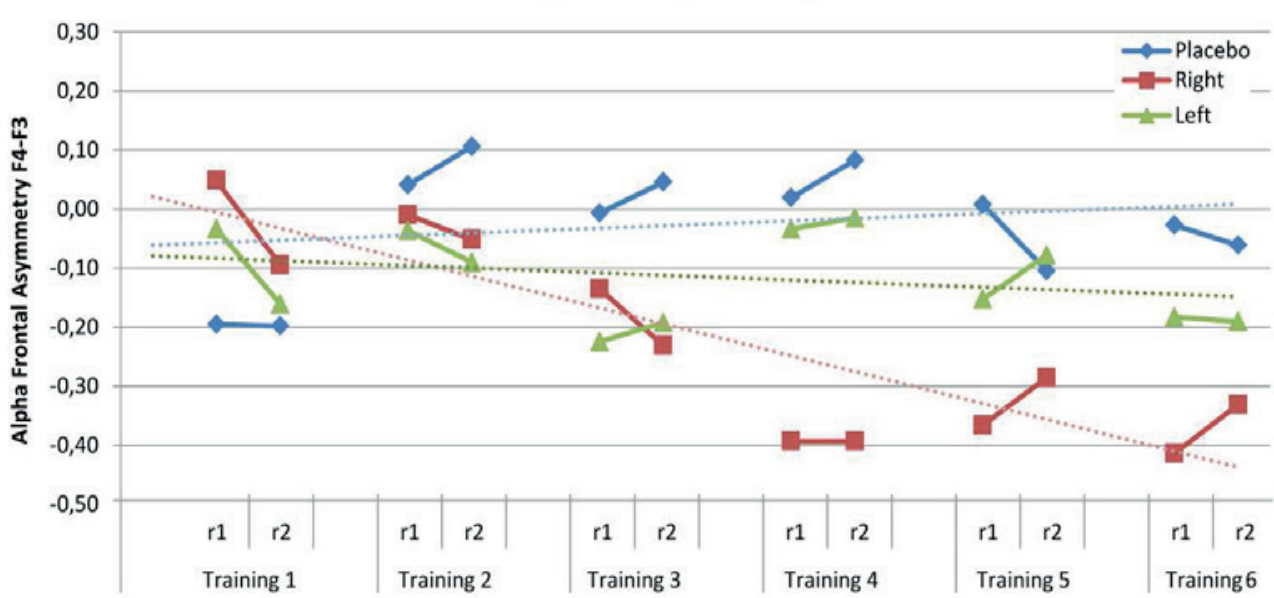

Figure 5.3 Frontal alpha asymmetry (F4-F3) during the rest measurements over 6 neurofeedback training sessions. Training group differentially changed the relative frontal asymmetry measured during rest between day 1 and 6 with a significant linear training effect for the right group (dashed red line). Positive alpha asymmetry scores indicate greater relative left than right frontal activity, while negative alpha asymmetry scores indicate greater relative right than left frontal activity. Abbreviations: $\mathrm{r} 1=$ asymmetry measurement before the neurofeedback training, $\mathrm{r} 2=$ asymmetry measurement after the neurofeedback training.

\section{Independence}

As the trainability analyses revealed that the trained frequency changed significantly in the right group, independence, stability, and interpretability, were subsequently assessed in the right group. The changes in relative frontal alpha asymmetry in the right group were compared to changes in asymmetry in other frequency bands or on other locations to assess the specificity of the frontal alpha asymmetry training. Both the theta and beta frequency bands were defined individually based on the IAF band. Similar to prior studies ${ }^{21}$ theta was defined as the frequency ranging 3 till $1 \mathrm{~Hz}$ below the lower bound of the IAF band. Beta was defined as the band ranging 1 to $3 \mathrm{~Hz}$ above the upper bound of the IAF band. The $1 \mathrm{~Hz}$ range below and above IAF was excluded to avoid frequency smearing. Fig. 5.4 displays the frequency spectra of training 1 and 6 for the beta, individual alpha frequency (IAF) and theta band and shows that the influence of the neurofeedback training on the spectra is most pronounced in the trained IAF band. This was corroborated by the linear contrasts with training $(1,2,3,4,5,6)$ and measurement (pre, post) as within subject variables (training $\times$ frequency interaction: $\left.F_{(1,16)}=13.80, p=.002 ; \eta^{2}{ }_{p}=.46\right)$. Follow-up analysis indicated that in the right group, frontal asymmetry in both the theta and beta band did not change over the six training sessions (training main effect theta: $F_{(1,17)}=0.05, p=.83$; Beta: $F_{(1,16)}=0.47, p=.51$ ).

The effect of the neurofeedback training in the right group on different electrode positions was assessed using linear contrasts with training $(1,2,3,4,5,6)$, location (F4-F3, C4-C3, P4-P3) and measurement (pre, post) as within subject factors. This revealed a trendlevel training $\times$ location interaction $\left(F_{(1,18)}=3.29, p=.08 ; \eta_{p}^{2}=.15\right)$. A follow-up analysis revealed that the regulation of frontal alpha asymmetry at F4-F3 in the right group was independent of changes in alpha asymmetry on the medial central and parietal locations (training main effect: $\mathrm{C} 4-\mathrm{C} 3: F_{(1,19)}=.001, p=.98$; P4-P3: $\left.F_{(1,19)}=0.73, p=.40\right)$. The location specificity of the frontal alpha asymmetry training was further investigated on the adjacent frontal locations F8-F7 and FC4-FC3. The pattern of the alpha asymmetry change was the same in adjacent frontal locations (training $\times$ location interaction: $F_{(1,19)}=1.38, p=.25$; training main effect $\left.F_{(1,19)}=15.36, p=.001 ; \eta^{2}=.45\right)$.

\section{Frequency independence in the right-trained group}

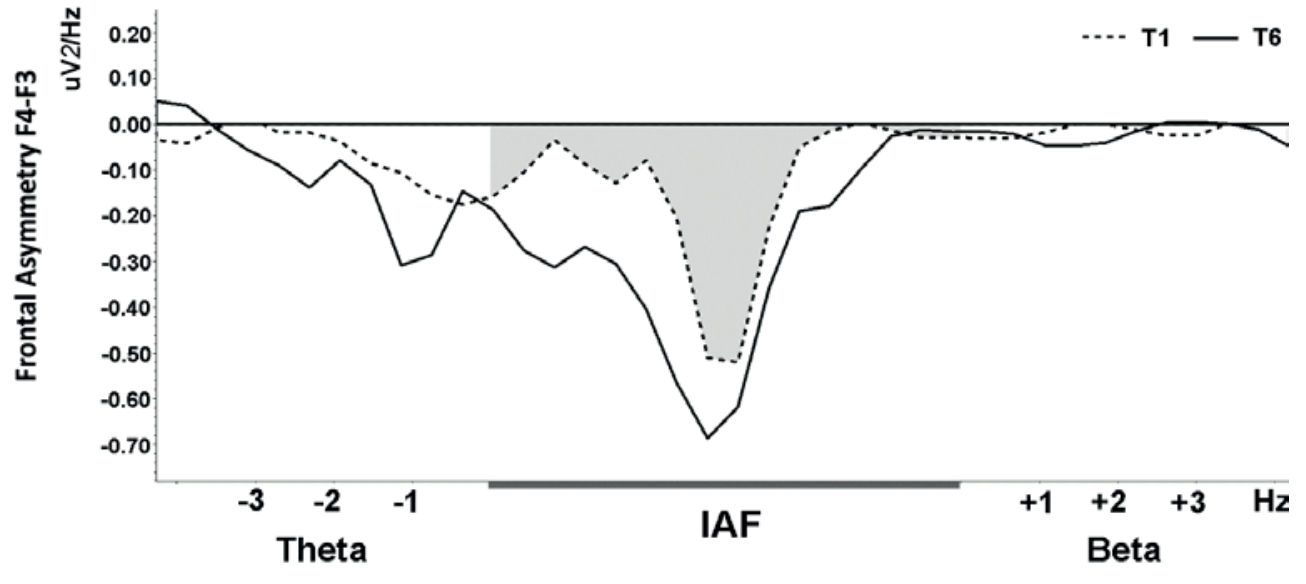

Figure 5.4 Frequency independence of the neurofeedback training in the right group. Frequency spectra of the baseline frontal asymmetry (F4-F3) of $\mathrm{T} 1$ (dashed lines) and of the baseline asymmetry of T6 (solid lines). The influence of the neurofeedback training on the spectra is most pronounced in the trained IAF band (grey area). There was no significant effect in theta (IAF -1 till -3 $\mathrm{Hz}$ ) and beta $(\mathrm{IAF}+1 \mathrm{till}+3 \mathrm{~Hz})$ band, thereby fulfilling the independence criterion. 


\section{Stability}

The stability of the frontal alpha asymmetry training in the right group was assessed one week and one month after the last neurofeedback training session. Fig. 5.5 shows that the relative frontal alpha asymmetry returned to baseline after the last neurofeedback training session. This was corroborated by the significant main effect of day $\left(F_{(3,57)}=2.88, p=.04 ; \eta^{2}{ }_{p}\right.$ $=.13$ ). Follow-up planned comparisons confirmed the expected changes in asymmetry between training 1 and $6(p=.002$ ), but the effect did not persist (training 1 versus one week: $p=.60$; training 1 versus one month $p=.57$ ).

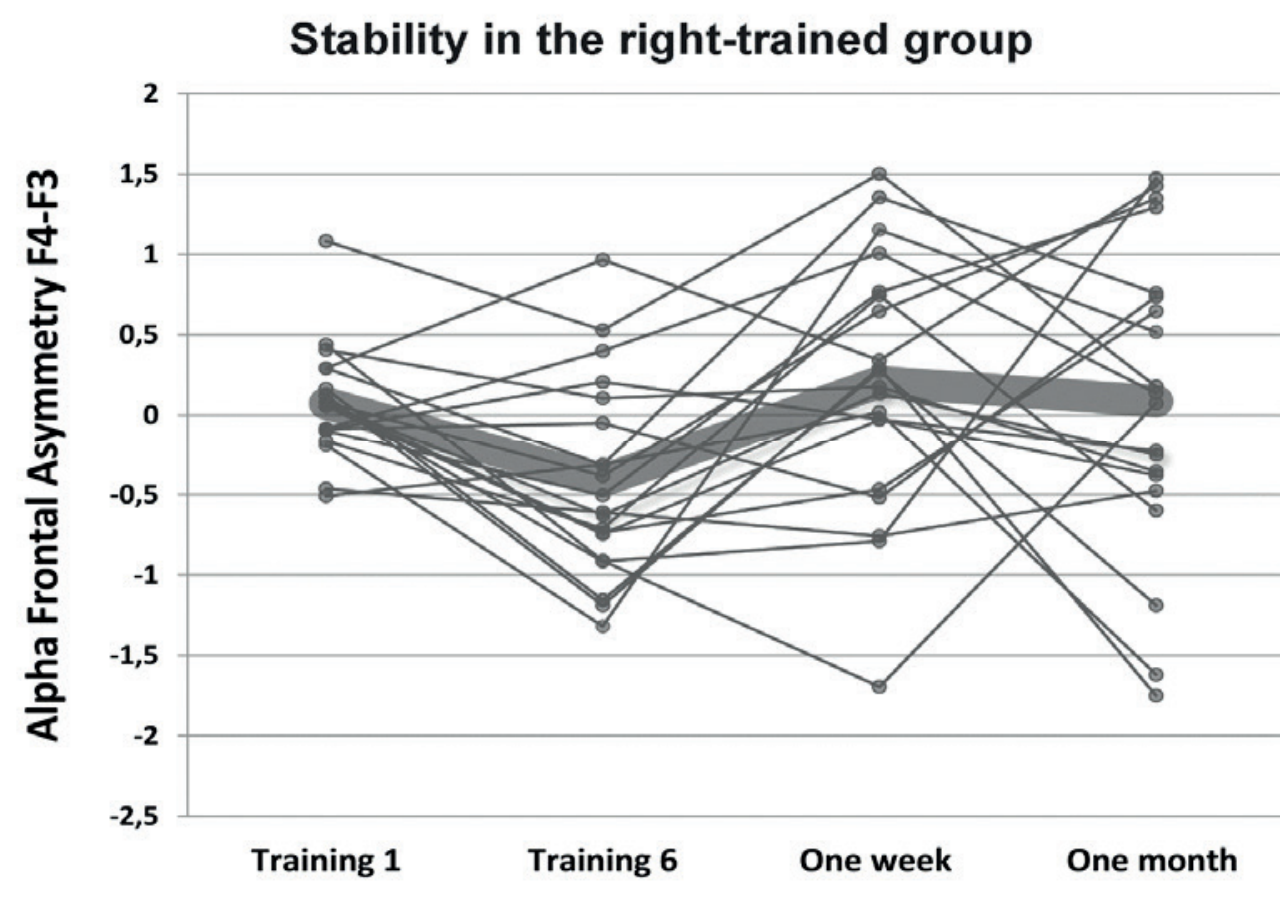

Figure 5.5 Stability of change in Frontal Alpha Asymmetry (F4-F3) measured during rest ( $r 1$ ) in the right group. Thick lines represent the group mean and thin lines display the individual stability one week and one month after the last neurofeedback training. Positive alpha asymmetry scores indicate greater relative left than right frontal activity while negative alpha asymmetry scores indicate greater relative right than left frontal activity.

\section{Interpretability}

The behavioural effect of the right neurofeedback training was assessed by testing the effect of training group on current mood using baseline corrected NA scores measured on the training days $(1,2,3,4,5,6)$ and on task-induced subjective and neuroendocrine stress response induced by the MAST on test days $(1,2,3)$. Table 5.1 displays the subjective stress response on the test days. Training group seemed to differentially affect the subjective stress response between test day 1 and 2 (test day $\times$ group trend-level interaction $F_{(2,48)}=2.34, p$ $=.10 ; \eta_{\mathrm{p}}^{2}=.09$ ). Simple-effect analyses revealed that the three training groups did not differ in task-induced subjective stress on test day $1\left(F_{(2,52)}=0.98, p=.38\right)$, but did differ at trendlevel on test day $2\left(F_{(2,52)}=2.49, p=.09 ; \eta^{2}=.09\right)$, with participants in the right neurofeedback group being more stressed than participants in the left group $(p=.03)$, while the left and placebo group did not differ and were less stressed on the second test day $(p=.23)$. Our interpretation is that the reduction in subjective stress response observed in the left and placebo groups is due to repeated stress induction in the laboratory. The neurofeedback training effect in the right group seemingly counteracted the reduction in subjective stress. No change over training days, nor neurofeedback group effects were found for current negative mood and neuroendocrine stress response (all $p s>.65$ ).

Table 5.1 Task-induced subjective stress response on the three test days. Mean + S.E.M.

\begin{tabular}{lllllll}
\hline & Right & & Left & \multicolumn{3}{l}{ Placebo } \\
\hline Test day 1 & 73.50 & 3.02 & 69.94 & 3.83 & 69.11 & 3.94 \\
Test day 2 & 72.50 & 3.21 & 59.28 & 5.99 & 62.77 & 5.36 \\
Test day 3 & 70.16 & 2.64 & 62.22 & 4.84 & 65.83 & 3.88 \\
\hline
\end{tabular}

\section{Individual differences in frontal asymmetry neurofeedback}

Fig. 5.6 displays the individual differences in frontal asymmetry during the rest measurements across training days as well as the average asymmetry score per group. It has been suggested that not everybody is able to learn how to self-regulate one's own cortical activity ${ }^{21,27,28}$. We defined responders as participants who produced a shift in relative asymmetry score (difference between $\mathrm{T} 6$ and $\mathrm{T} 1$ ) during rest $(\mathrm{r} 1)$ in the desired direction (i.e., right relative more negative, while left relative more positive alpha asymmetry at $\mathrm{T} 6$ than at T1) 


\section{Individual differences and neurofeedback group}

In the left group, 8 participants were classified as responders and 10 as non-responders. In the right group, 15 participants were responders and 5 were non-responders. This difference in relative proportion of responders per training group was statistically significant $\left(X^{2}(1)=\right.$ $3.70, p=.05)$. Based on the odds ratio, participants were 3.8 times more likely to change their frontal asymmetry in the desired direction in the right than in the left training group. This result is consistent with the above results in the group as-a-whole, showing only a significant effect in the right group. There were no differences between men and women with respect to the number of responders per group (right: $X^{2}{ }_{(1)}=0.61, p=.44$; Left: $X^{2}(1)=0.18, p=.67$ ).

\section{Individual differences and trainability, interpretability and stability}

The validity of the classification into responders and non-responders based on the difference in rest asymmetry over training sessions was assessed on independent measures. A difference between responders and non-responders in changing frontal asymmetry during the feedback was assessed with training $(1,2,3,4,5)$ and block (block 1, block 2, block 3 ) as linear subject variables and group (left, right) and responder (responder, nonresponder) as between subject variables. This revealed a trend-level training $\times$ responder $\left(F_{(1,34)}=3.72, p=.06 ; \eta_{\mathrm{p}}^{2}=.10\right)$ and a group $\times$ responder interaction $\left(F_{(1,34)}=5.55, p=.02 ; ; \eta^{2}{ }_{\mathrm{p}}\right.$ $=.14)$. Follow-up analysis revealed a trend-level responder main effect for the right group $\left(F_{(1,18)}=3.89, p=.06 ; \eta^{2}{ }_{p}=.18\right)$ and a trend-level training $\times$ responder interaction $\left(F_{(1,16)}=3.67\right.$, $p=.07 ; \eta_{\mathrm{p}}^{2}=.19$ ) for the left group (see Fig. 5.7) suggesting that responders and nonresponders differed in changing frontal asymmetry during the neurofeedback blocks across training sessions. For both groups, no differences between responders and non-responders with regard to subjective stress (test day 1 versus one week: day $\times$ responder interaction $p$ s $>$.24) or stability (training 1 versus one week: day $\times$ responder interaction $p s>.21$; training 1 versus one month: day $\times$ responder interaction $p s>.11$ ) were found.
Alpha Frontal Asymmetry F4-F3

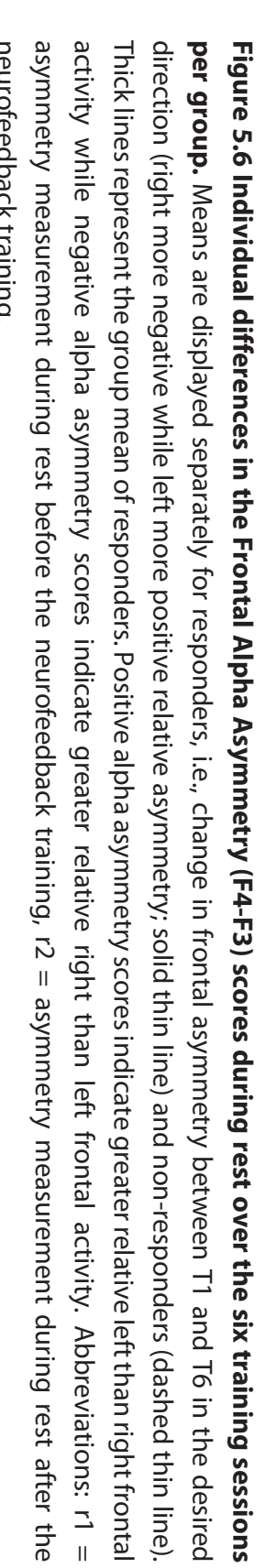

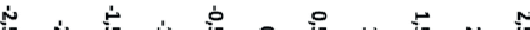

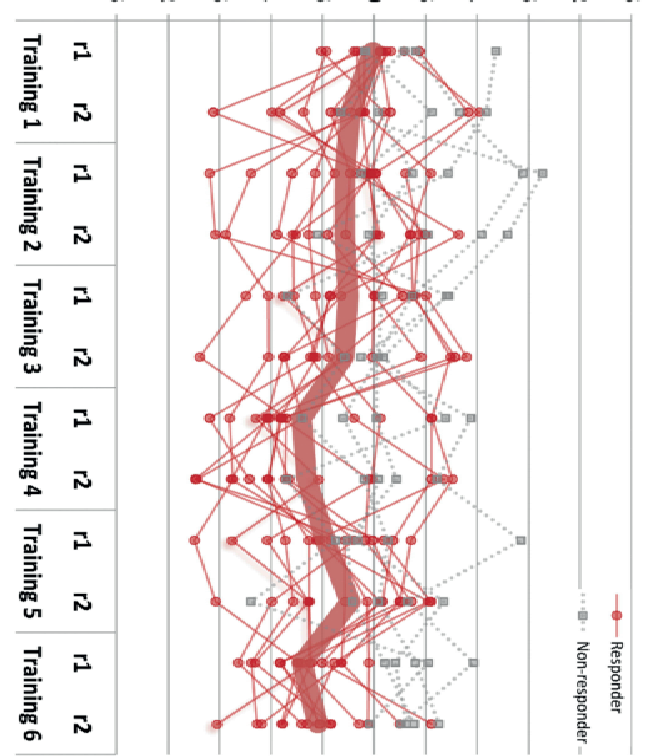

Alpha Frontal Asymmetry F4-F3

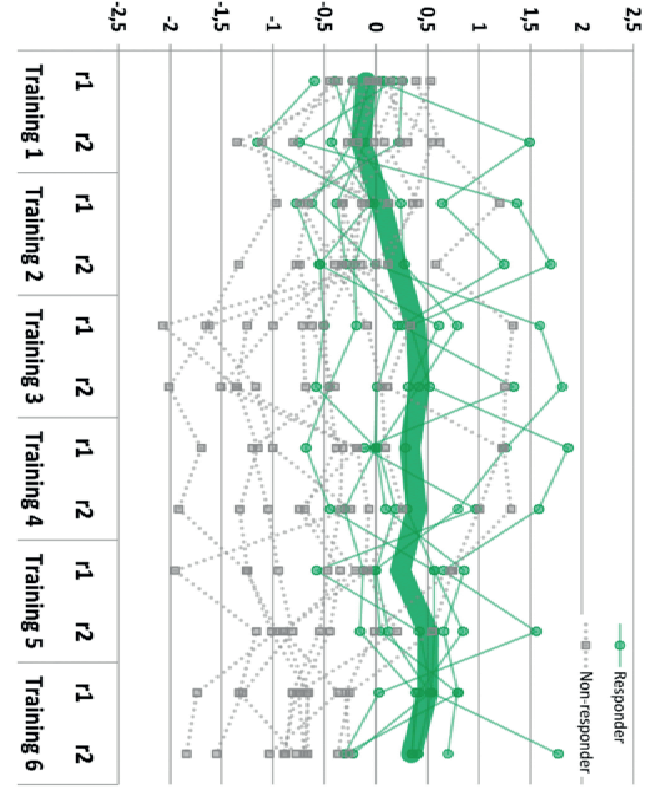

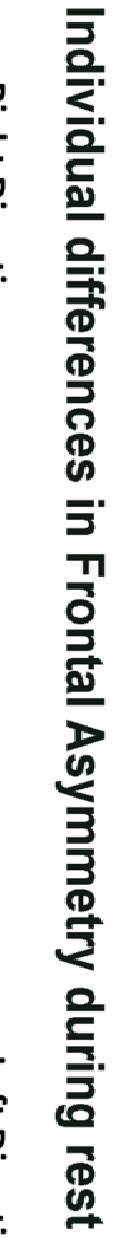




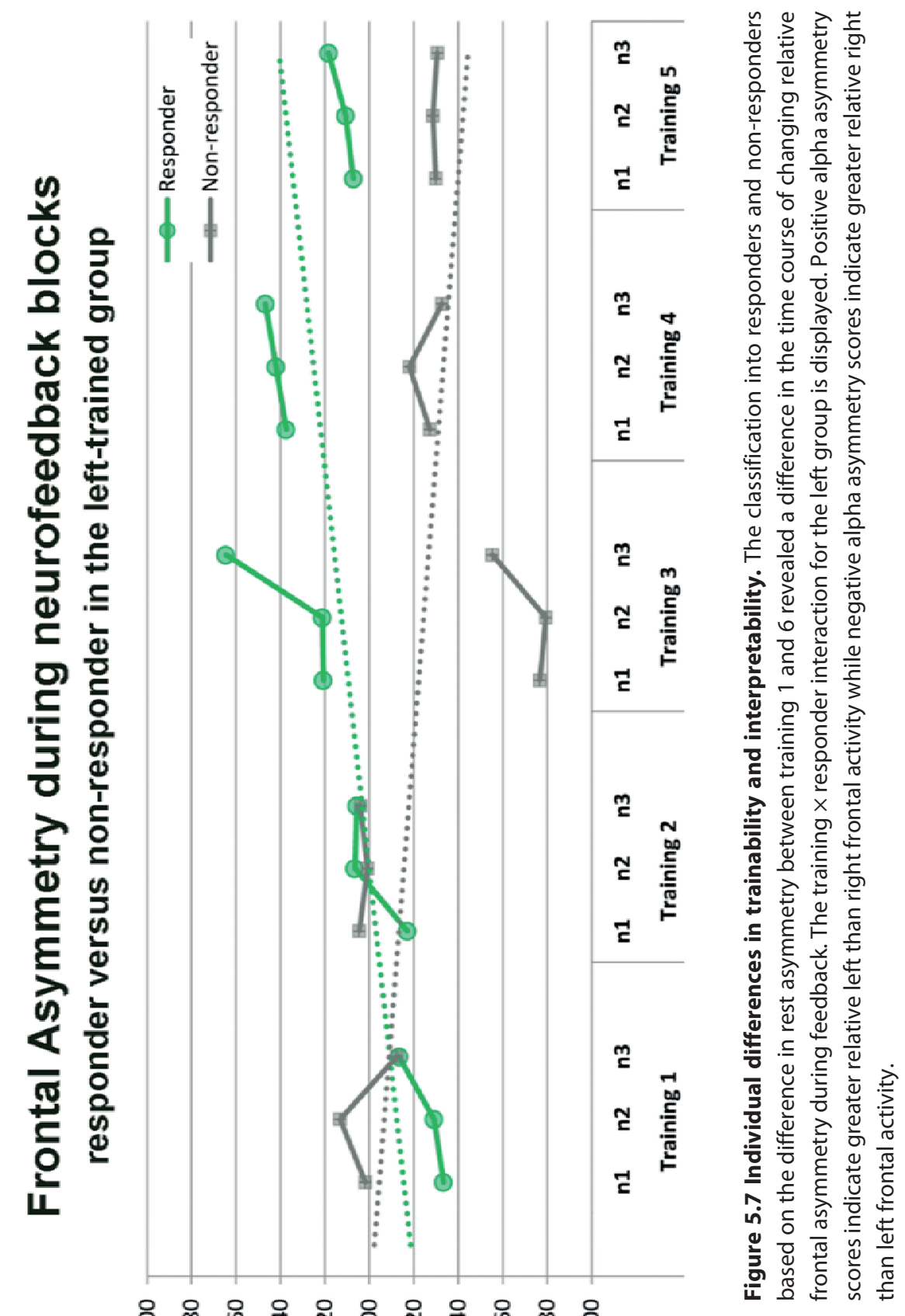

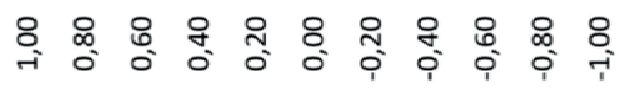

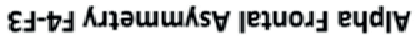

\section{Discussion}

Frontal alpha asymmetry is assumed to be associated with psychopathology and individual differences in emotional responding. Neurofeedback is a tool that can be used to change frontal alpha asymmetry and could, therefore, prove to be a practical intervention option to increase resilience. The current study assessed the trainability, interpretability, stability, and specificity of a neurofeedback protocol that was designed to change relative frontal alpha asymmetry. The neurofeedback protocol that was developed and evaluated in the current work uses real-time eye-corrected and average mastoid-referenced individual alpha frequency data as the basis for frontal asymmetry feedback. Furthermore, by including a placebo group and follow-up measurements one week and one month later, the current study extends the knowledge that was accumulated in previous alpha asymmetry neurofeedback studies.

We first assessed the effectiveness of the neurofeedback protocol for the whole sample by evaluating the change in relative frontal asymmetry in the alpha band. For the right group, a linear increase of relative frontal asymmetry during rest over training sessions was found, suggesting transfer of the previous learning experience to the next training. This change in relative asymmetry to the right was only found during the rest measurement and not during the feedback itself. This could be due to the fact that learning from feedback involves high levels of cognitive effort and attention. Importantly, both cognitive effort and outward attention are accompanied by alpha suppression ${ }^{49}$, which may decrease the sensitivity to detect asymmetry changes. Furthermore, it is possible that neurofeedback is more effective in changing tonic rather than phasic alpha asymmetry as tonic alpha changes occur at a slower rate ${ }^{28}$. It has been shown that manipulation of alpha oscillations during rest is related to high performance levels and alpha suppression during cognitive tasks ${ }^{50}$.

For task-induced subjective stress responses between test day 1 and 2, we found a reduction in subjective stress on test day 2 in the left and placebo group. Participants in the right group, on the other hand, felt equally stressed on both test days. The absence of a decrease in the right group could result from the neurofeedback training and its shift in relative frontal asymmetry to the right. This interpretation is consistent with the idea that asymmetrical activation of the prefrontal cortex plays a role in adaptive coping, with a right lateralised withdrawal system involved in negative affect $^{2-4}$. These results mirror our previous fMRI finding of an association between reduced task-induced subjective stress and 
enhanced connectivity between the amygdala and left dorsolateral prefrontal cortex during rest (see Chapter 2$)^{51}$. No changes in mood were observed over the six training days. Our finding that mood is seemingly unaffected by alpha asymmetry neurofeedback training is well in line with those of previous studies ${ }^{22,23}$, which might imply that unprovoked selfreported mood is not sufficiently sensitive. Several frontal alpha asymmetry neurofeedback studies in patient samples found reduced depressive symptoms ${ }^{17,18,52,53}$. Some authors also suggested that in healthy participants, asymmetrical frontal activity acts as a moderator of mood responses when the appropriate emotional stimuli are presented ${ }^{2,54}$. This is supported by studies in healthy participants demonstrating that frontal asymmetry is not related to unprovoked affective states ${ }^{55,56}$ but see ${ }^{57}$. Note in passing that the precise motivational circumstances determining the association with affective responding is not yet fully understood and require further study ${ }^{45}$. Training direction did not differentially change the task-induced cortisol response between test day 1 and 2 . This corroborates our previous findings of no stress-induced change in frontal asymmetry (see Chapter 4) ${ }^{9}$. Moreover, high frequency rTMS intended to change relative frontal activity had no effect on cortisol levels in an unchallenged situation in healthy participants ${ }^{58}$. However, that same procedure did change the cortisol response in experimentally stressed women ${ }^{59}$, suggesting that the moderating role of baseline frontal alpha activity on the fight-or-flight neuroendocrine response is state-dependent.

The changes in relative frontal alpha asymmetry in the right group occurred independent of other frequency bands and were also found at adjacent frontal locations, but not at central and parietal locations. This confirms the specificity of the results obtained by the current study's neurofeedback protocol. Similarly, Harmon-Jones and collegues ${ }^{23}$ used a two-day alpha frontal asymmetry protocol and found a specific effect between the increase and decrease relative frontal asymmetry group on F4/F3, but not on P4/P3 and, more recently, Peeters and colleagues ${ }^{24}$ also reported frequency specific effects after one day of frontal alpha asymmetry training. Other studies using alpha amplitude neurofeedback protocols, however, did find changes beyond the target frequency and location ${ }^{19,32,60,61}$. This suggests that the specificity of the training depends on the type of alpha neurofeedback protocol used (e.g., asymmetry versus amplitude training). The changes in the right group did not persist at one week and one month follow-up measurements. While such a return to baseline has also been reported by previous studies ${ }^{22,24}$, it disagrees with findings of Choi and colleagues ${ }^{17}$ showing a stable effect of leftsided frontal asymmetry training after one month in depressive patients. Our data suggests that although we can effectively train healthy people to display more relative right-sided asymmetry, these effects seem to be short-lived.

In line with previous studies using different alpha neurofeedback protocols ${ }^{21,27,28}$, the current data show that not all participants learned from the neurofeedback how to modulate their cortical activity. Trained in the left group, $44 \%$ of the participants demonstrated a less negative rest asymmetry score while after training in the right group $75 \%$ demonstrated a more negative rest asymmetry score. These data support Allen and colleagues ${ }^{22}$ observation that it is easier to increase right- rather than left-relative frontal asymmetry in healthy participants using EEG neurofeedback. We assessed the validity of the classification into responders and non-responders based on the difference in rest asymmetry between training 1 and 6 based in terms of trainability during the neurofeedback, interpretability, and stability. While we did not find an effect of the neurofeedback training on relative frontal asymmetry during the feedback for the whole group analysis, the resting-EEG-based classification did differentiate responders from nonresponders during the feedback training. The predictive value of changes in resting alpha oscillations for the learning ability of alpha neurofeedback was also shown in the study of Wan and colleagues ${ }^{62}$. Previous alpha neurofeedback studies defined their own responder classification criteria and did not assess the validity of the employed classification on independent measures. Our finding that individual differences in trainability is dependent on training direction, with participants in the right direction being more likely to change their frontal asymmetry in the desired direction, indicates that it is important to make a responder versus non-responder distinction. By doing so, the current and future studies may gain further insight in the processes involved in training self-regulation. Also, for therapeutic applications it is important to unambiguously define a training criterion. A responder criterion would enable to determine the end point of the training or could help to identify participants who do not respond to neurofeedback treatment as early as possible so that no additional and unnecessary training is imposed upon the unresponsive participants.

To be truly effective, feedback should be contingent on brain activity alone. This has implications for the methodological design of the neurofeedback training. For example, since eye movement artefacts influence the EEG activity, especially at the frontal sites and 
in the alpha band, it is important to correct them real-time when using a frontal alpha asymmetry neurofeedback training protocol ${ }^{63,64}$. Moreover, computerized linked mastoids (i.e., average of $\mathrm{A} 1+\mathrm{A} 2$ ) reference has a somewhat superior signal-to-noise ratio compared to $C z$, which has been used in previous frontal alpha asymmetry neurofeedback protocols ${ }^{2,42}$. The current neurofeedback protocol calculated real-time averages of both references and corrected eye blinks online and, thus, represents an important step forward to making instrumental conditioning of brain rhythms more specific.

In sum, individual frontal alpha frequency neurofeedback resulted in a change in relative frontal asymmetry at rest in participants in the right group, and this change in relative frontal alpha asymmetry seemed to affect subjective stress. Moreover, we found that the trainability in the right group was specific with regard to frequency band and location Individual differences in trainability dependent on training group were found, with participants in the right group being more likely to change their frontal asymmetry in the desired direction. The individual differences in trainability were also reflected in the ability to change frontal asymmetry during the feedback. Whether the current neurofeedback is also capable of eliciting reliable effects at the behavioural level, which would be especially useful in clinical populations, remains open to further empirical testing.

\section{References}

Harmon-Jones, E., Gable, P.A. \& Peterson, C.K. (2010). The role of asymmetric frontal cortical activity in emotion-related phenomena: a review and update. Biological Psychology, 84, 451-462.

2 Coan, J.A. Allen, J.J.B. \& McKnight, P.E. (2006). A capability model of individual differences in frontal EEG asymmetry. Biological Psychology, 72, 198-207.

3 Davidson, R.J. (1998). Affective style and affective disorders: Perspectives from affective neuroscience. Cognition \& Emotion, 12, 307-330.

4 Tomarken, A.J., Davidson, R.J., Wheeler R.E. \& Doss, R.C. (1992). Individual differences in anterior brain asymmetry and fundamental dimensions of emotion. Journal of Personality and Social Psychology, 62, 676-687.

5 Davidson, R.J. (2004). What does the prefrontal cortex "do" in affect: perspectives on frontal EEG asymmetry research. Biological Psychology, 67, 219 233.

6 Sullivan, R.M. \& Gratton, A. (2002) Prefrontal cortical regulation of hypothalamic-pituitary-adrenal hypothalamic-pituitary-adrenal
function in the rat and implications for psychopathology: side matters. Psychoneuroendocrinology, 27, 99-114.

7 Papousek, I., Reiser, E.M., Weber, B. Freudenthaler, H.H. \& Schulter, G. (2012). Frontal brain asymmetry and affective flexibility in an emotiona contagion paradigm. Psychophysiology, 49, 489-498.

8 Jackson, D.C. et al. (2003). Now you feel it, now you don't: Frontal brain electrica asymmetry and individual differences in emotion regulation. Psychological Science, 14, 612-617.
9 Quaedflieg, C.W.E.M., Meyer, T., Smulders, F.T.Y. \& Smeets, T. (2015). The functional role of individual-alpha based frontal asymmetry in stress responding. Biological Psychology, 104, 75-81.

10 Thibodeau, R., Jorgensen, R.S. \& Kim, S. (2006). Depression, anxiety, and resting frontal EEG asymmetry: a meta-analytic review. Journal of Abnormal Psychology 115, 715-729.

11 Moscovitch, D.A. et al. (2011). Frontal EEG asymmetry and symptom response to cognitive behavioural therapy in patients with social anxiety disorder. Biological Psychology, 87, 379-385.

12 Loo, C.K. \& Mitchell, P.B. (2005). A review of the efficacy of transcranial magnetic stimulation (TMS) treatment for depression, and current and future strategies to optimize efficacy. Journal of Affective Disorders, 88, 255-267.

13 Pallanti, S. \& Bernardi, S. (2009). Neurobiology of repeated transcranial magnetic stimulation in the treatmen of anxiety: a critical review. International Clinical Psychopharma-cology, 24, 163173.

14 Heinrich, H., Gevensleben, H. \& Strehl, U. (2007). Annotation: Neurofeedback train your brain to train behaviour. Journal of Child Psychology and Psychiatry, 48, 3-16.

15 Arns, M., Heinrich, H. \& Strehl, U. (2014). Evaluation of neurofeedback in ADHD: The long and winding road. Biological Psychology, 95, 108-115.

16 van Dongen-Boomsma, M., Vollebregt, M.A., Slaats-Willemse, D. \& Buitelaar, J.K. (2013). A randomized placebocontrolled trial of electroencephalographic (EEG) neurofeedback in children with attention- 
deficit/hyperactivity disorder. Journal of Clinical Psychiatry, 74, 821-827.

17 Choi, S.W. et al. (2011). Is alpha wave neurofeedback effective with randomized clinical trials in depression? A pilot study. Neuro-psychobiology, 63 , 43-51.

18 Peeters, F., Oehlen, M., Ronner, J., van Os, J. \& Lousberg, R. (2014). Neurofeedback as a treatment for majo depressive disorder-a pilot study. PloS One, 9, e91837.

19 Gruzelier, J.H. (2014). EEG neurofeedback for optimising performance. III: a review of methodological and theoretical considerations. Neuroscience and Biobehavioural Reviews, 44, 159-182.

20 Lofthouse, N., Arnold, L. E., Hersch, S. Hurt, E. \& DeBeus, R. (2012). A review of neurofeedback treatment for pediatric ADHD. Journal of Attention Disorders, 16 , 351-372.

21 Zoefel, B., Huster, R.J. \& Herrmann, C. S. 2011). Neurofeedback training of the upper alpha frequency band in EEG improves cognitive performance. Neuroimage, 54, 1427-1431.

22 Allen, J.J., Harmon-Jones, E. \& Cavender, J.H. (2001). Manipulation of frontal EEG asymmetry through biofeedback alters self-reported emotional responses and facial EMG. Psychophysiology, 38, 685693.

23 Harmon-Jones, E., Harmon-Jones, C., Fearn, M., Sigelman, J.D. \& Johnson, P. (2008). Left frontal cortical activation and spreading of alternatives: Tests of the action-based model of dissonance. Journal of Personality and Social Psychology, 94, 1-15.

24 Peeters, F., Ronner, J., Bodar, L., van Os, J. \& Lousberg, R. (2014). Validation of neurofeedback paradigm: manipulating frontal EEG alpha-activity and its impact on mood. International
Journal of Psycho-physiology, 93, 116-

120.

25 Doppelmayr, M., Klimesch, W. Pachinger, T. \& Ripper, B. (1998). Individual differences in brain dynamics: important implications for the calculation of event-related band power. Biological Cybernetics, 79, 49-57.

26 Klimesch, W., Schimke, H. \& Pfurtscheller, G. (1993). Alpha frequency, cognitive load and memory performance. Brain Topography, 5, 241 251.

27 Dekker, M.K., Sitskoorn, M.M., Denissen A.J. \& van Boxtel, G.J. (2014). The timecourse of alpha neurofeedback training effects in healthy participants. Biological Psychology, 95, 70-73.

28 Hanslmayr, S., Sauseng, P., Doppelmayr M., Schabus, M. \& Klimesch, W. (2005) Increasing individual upper alpha power by neurofeedback improves cognitive performance in human subjects. Applied Psychophysiology and Biofeedback, 30, 1-10.

29 Kouijzer, M.E., van Schie, H.T., Gerrits, B.J., Buitelaar, J.K. \& de Moor, J.M. (2013). Is EEG-biofeedback an effective treatment in autism spectrum disorders? A randomized controlled trial. Applied Psychophysiology and Biofeedback, 38, 17-28.

30 Kudielka, B.M., Hellhammer, D.H. \& Wust, S. (2009). Why do we respond so differently? Reviewing determinants of human salivary cortisol responses to challenge. Psychoneuroendocrinology, $34,2-18$

31 Klimesch, W. (1999). EEG alpha and theta oscillations reflect cognitive and memory performance: a review and analysis. Brain Research Reviews, 29, 169195.

32 van Boxtel, G.J.et al. (2012). A novel selfguided approach to alpha activity training. International Journal of Psychophysiology, 83, 282-294.

33 Velo, J.R., Stewart, J.L., Hasler, B.P. Towers, D.N. \& Allen, J.J.B. (2012) Should it matter when we record? Time of year and time of day as factors influencing frontal EEG asymmetry. Biological Psychology, 91, 283-291.

34 Quaedflieg, C.W.E.M., Schwabe, L. Meyer, T. \& Smeets, T. (2013). Time dependent effects of stress prior to encoding on event-related potentials and 24-h delayed retrieval. Psychoneuroendocrinology, 38, 30573069.

35 Smeets, T. et al. (2012). Introducing the Maastricht Acute Stress Test (MAST): A quick and non-invasive approach to elicit robust autonomic and glucocorticoid stress responses. Psychoneuroendocrinology, 37, 19982008.

36 Pruessner, J.C., Kirschbaum, C. Meinlschmid, G. \& Hellhammer, D.H. (2003). Two formulas for computation of the area under the curve represent measures of total hormone concentration versus time-dependen change. Psychoneuroendocrinology, 28, 916-931.

37 Watson, D., Clark, L.A. \& Tellegen, A (1988). Development and validation of brief measures of positive and negative affect: the PANAS scales. Journal of Personality and Social Psychology, 54, 1063-1070.

38 Goldman, R.I., Stern, J.M., Engel, J. \& Cohen, M.S. (2002). Simultaneous EEG and fMRI of the alpha rhythm. Neuroreport, 13, 2487-2492.

39 Laufs, H. et al. (2003). EEG-correlated fMRI of human alpha activity. Neuroimage, 19, 1463-1476.

40 Pfurtscheller, G., Stancak, A. \& Neuper, C. (1996). Event-related synchronization (ERS) in the alpha band -
An electrophysiological correlate of cortical idling: A review. International Journal of Psycho-physiology, 24, 39-46.

41 Pizzagalli, D.A., Sherwood, R.J. Henriques, J.B. \& Davidson, R.J. (2005) Frontal brain asymmetry and reward responsiveness - A source-localization study. Psychological Science, 16, 805 813.

42 Hagemann, D. (2004). Individual differences in anterior EEG asymmetry: methodological problems and solutions. Biological Psychology, 67, 157 182.

43 Semlitsch, H.V., Anderer, P., Schuster, P. \& Presslich, O. (1986). A solution for reliable and valid reduction of ocula artefacts, applied to the P300 ERP. Psychophysiology, 23, 695-703.

44 Delorme, A. \& Makeig, S. (2004). EEGLAB: an open source toolbox for analysis of single-trial EEG dynamic including independent componen analysis. Journal of Neuroscience Methods, 134, 9-21.

45 Meyer, T. et al. (2014). Frontal EEG asymmetry as predictor of physiologica responses to aversive memories. Psychophysiology, 51, 853-865.

46 Allen, J.J.B., Coan, J.A. \& Nazarian, M. (2004). Issues and assumptions on the road from raw signals to metrics of frontal EEG asymmetry in emotion. Biological Psychology, 67, 183-218.

47 Rosnow, R.L. \& Rosenthal, R. (1989). Statistical Procedures and the Justification of Knowledge in Psychological Science. American Psychologist, 44, 1276-1284.

48 Fritz, C.O., Morris, P.E. \& Richler, J.J. (2012). Effect Size Estimates: Curren Use, Calculations, and Interpretation. Journal of Experimental PsychologyGeneral, 141, 2-18.

49 Klimesch, W., Doppelmayr, M Russegger, $H_{.}$, Pachinger, T. \& 
Schwaiger, J. (1998). Induced alpha band power changes in the human EEG and attention. Neuroscience Letters, 244 73-76.

50 Klimesch, W., Sauseng, P. \& Gerloff, C. (2003). Enhancing cognitive performance with repetitive performance with repetitive
transcranial magnetic stimulation at human individual alpha frequency. European Journal of Neuroscience, 17, 1129-1133.

51 Quaedflieg, C.W.E.M. et al. (2015). Temporal dynamics of stress-induced alternations of intrinsic amygdala connectivity and neuroendocrine levels. PloS One, 10, e0124141.

52 Baehr, E., Rosenfeld, J.P., Baehr, R. \& Earnest, C. (1998). Comparison of two EEG asymmetry indices in depressed patients vs. normal controls. International Journal of Psychophysiology, 31, 89-92.

53 Hammond, D.C. (2005). Neurofeedback treatment of depression and anxiety. Journal of Adult Development, 12, 131 137.

54 Coan, J.A. \& Allen, J.J.B. (2004). Frontal EEG asymmetry as a moderator and mediator of emotion Biological Psychology, 67, 7-49.

55 Harmon-Jones, E. \& Allen, J.J.B. (1997) Behavioural activation sensitivity and resting frontal EEG asymmetry: Covariation of putative indicators related to risk for mood disorders. Journal of Abnormal Psychology, 106, 159-163.

56 Sutton, S.K. \& Davidson, R.J. (1997). Prefrontal brain asymmetry: A biological substrate of the behavioural approach and inhibition systems. Psychological Science, 8, 204-210.

57 Hagemann, D., Naumann, E., Becker, G., Maier, S. \& Bartussek, D. (1998). Fronta brain asymmetry and affective style: a conceptual replication. Psychophysiology, 35, 372-388.

58 Baeken, C., Vanderhasselt, M.A. \& De Raedt, R. (2011). Baseline 'state anxiety' influences HPA-axis sensitivity to on sham-controlled HF-rTMS session applied to the right dorsolatera prefrontal cortex. Psychoneuroendocrinology, 36, 60-67.

59 Baeken, C. et al. (2014). One left dorsolateral prefrontal cortical HF-rTMS session attenuates HPA-system sensitivity to critical feedback in healthy females. Neuropsychologia, 57, 112-121.

60 Egner, T., Zech, T.F. \& Gruzelier, J.H. (2004). The effects of neurofeedback training on the spectral topography of

the electroencephalogram. Clinical Neurophysiology, 115, 2452-2460.

61 Enriquez-Geppert, S. et al. (2014). Modulation of frontal-midline theta by neurofeedback. Biological Psychology, 95, 59-69.

62 Wan, F., Nan, W., Vai, M.I. \& Rosa, A. (2014). Resting alpha activity predicts learning ability in alpha neurofeedback. Frontiers in Human Neuroscience, 8, 500.

63 Huster, R.J., Mokom, Z.N., EnriquezGeppert, S. \& Herrmann, C.S. (2014). Brain-computer interfaces for EEG neurofeedback: peculiarities and solutions. International Journal of Psychophysiology, 91, 36-45.

64 Sherlin, L. et al. (2011). Neurofeedback and basic learning theory: Implications for research and practice. Journal of Neurotherapy: Investigations in Neuromodulation, Neurofeedback and Applied Neuroscience, 15, 292-304. 


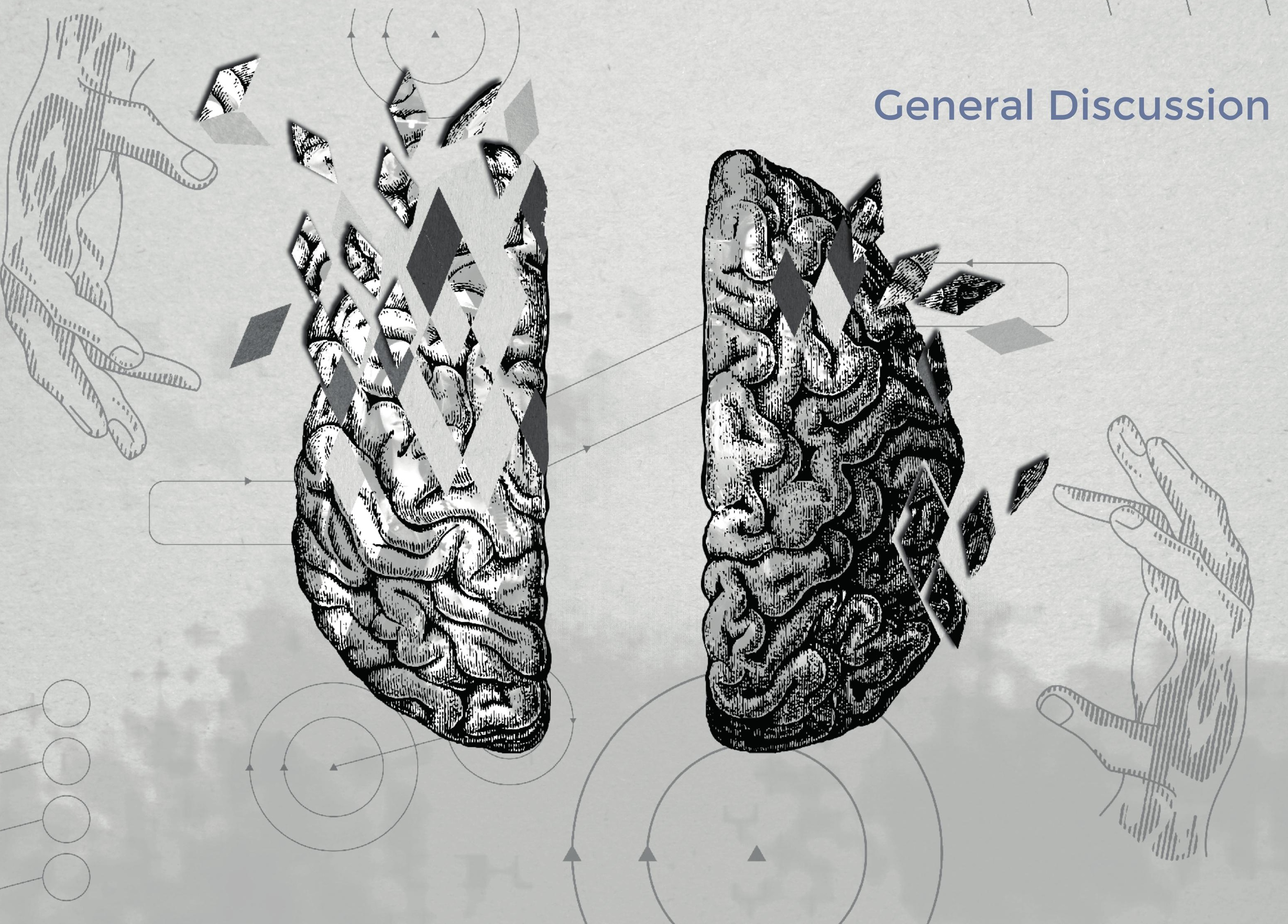


A wide range of events can provoke stress, though the amount of stress that is experienced in any given situation can differ between individuals. The ability to cope with stressful events is defined by individual differences in resilience to stress events. The work presented in this dissertation aimed to identify the changes in neural responding that underlie adaptability after exposure to acute stress and that thus characterize stress resilience. To this end, we used an interdisciplinary approach that combines psychophysiology and neuroimaging methods. In particular, we used functional magnetic resonance imaging (fMRI) and electroencephalography (EEG) measurements and developed the imaging Maastricht Acute Stress Test (iMAST). In Part 1 of this thesis, "Imaging the stressed brain", we investigated the effects of acute stress on functional connectivity of the brain and on the neural correlates of memory formation. In Part $\mathbf{2}$ of the thesis, "Brain asymmetry and stress resilience", we tested the functional role of frontal alpha asymmetry in stress responding and explored the possibility to modify cortical brain activity via neurofeedback to target stress resilience. The following sections first summarize the key findings and insights in the underlying neural mechanisms of stress resilience that are presented in Part 1 of this thesis. This is followed by a summary of the chapters described in Part 2 of this thesis highlighting insights that are relevant for novel treatments based on the real-time analysis of brain activity. Subsequently, based on this dissertation's findings, new avenues for research on individual differences in stress resilience are suggested.

\section{Imaging the stressed brain}

It is generally accepted that our brain regulates the acute stress response and that acute stress in turn affects neuronal activity in the brain. The response to acute stress is multidimensional and the activation of this integrated neuroendocrine system is time specific. It is known that the interaction between fast-acting catecholamines and slow-acting glucocorticoids influences neuronal and cognitive processing in the human brain ${ }^{1-3}$. However, how catecholamines and glucocorticoids change neural processing in a spatial and time-dependent matter in response to acute stress is not yet fully understood. In Chapters $\mathbf{1}$ and $\mathbf{2}$, fMRI was used to investigate the temporal and spatial changes in neural responding that underlie flexible adaptability after acute stress. Given the scarcity of paradigms that reliably evoke a glucocorticoid response in the neuroimaging environment, we developed and validated a paradigm to induce acute stress in the MRI scanner. In the 
following sections, the imaging stress paradigm is first summarized followed by several key findings regarding the moderating role of glucocorticoids on the change in amygdala resting state functional connectivity. The stress-induced changes in amygdala connectivity during the acute stress phase are first discussed, followed by the changes during the early recovery phase.

\section{Imaging the stressed brain - use the iMAST}

Systematically investigating the mediating role of the stress hormone cortisol in the brain's response to a stressor requires a paradigm that effectively activates the HPA-axis stress response. Chapter 1 presents a novel fMRI compatible stress paradigm consisting of several exposures to cold-pressor stress and various mental arithmetic challenges along with socialevaluative pressure to reliably elicit solid cortisol responses. Cortisol increases of at least 1.5 $\mathrm{nmol} / \mathrm{l}$ following exposure to a laboratory stressor, compared with baseline, are currently defined as an indication that the stressor indeed activates the HPA-axis ${ }^{4}$. In healthy participants, we observed that the iMAST successfully elicited cortisol responses of at least $1.5 \mathrm{nmol} / \mathrm{L}$ in almost $70 \%$ of the participants, making the iMAST one of the most powerfu tools currently available to elicit HPA-axis stress responses in a neuroimaging environment. Additionally, the iMAST was also capable of eliciting strong autonomic stress responses as determined by changes in salivary alpha-amylase. Adding to the idea that the iMAST is one of the most powerful tools to elicit autonomic and cortisol responses in fMRI studies, is the fact that previous neuroimaging studies generally classified participants as responders when they displayed any positive departure from baseline cortisol levels $s^{5-7}$. The effectiveness of the iMAST is most likely due to the unique combination of a physical and a psychological stressor. Monitoring of and verbal negative feedback on participants' performance while in the scanner creates a social-evaluative threat component that triggers the HPA-axis via activation of the prefrontal cortex (PFC), thalamus, and limbic structures ${ }^{8,9}$.

It is important to expose participants to acute stress whilst they are inside the scanner to be able to capture the effects of the fast acting autonomic nervous system and the slow acting HPA-axis on brain processing. Moreover, inducing stress inside the scanner avoids possible confounding by stress elicited by the scanning environment itself. Paramount among the imaging stress tests so far is the Montreal Imaging Stress Task (MIST) ${ }^{10}$. The MIST uses mental arithmetic's in combination with computer generated negative feedback. Thus, the MIST is a motivated performance task that consists of multiple components including cognitive processing during the math task, social evaluative threat, and the processing of failure, that are hard to model separately. Consequently, the activation patterns could reflect differences in task performance rather than individual differences in acute stress processing. The current design of the iMAST allows for investigating the post-stress contribution of different brain areas during stress regulation while participants are counting out loud. This resembles behavioural laboratory stress research that typically investigates the effects of stress on cognitive processes after stress induction.

\section{Imaging the stressed brain - the immediate stress phase}

The response to acute stress is multi-faceted. Initially, during the immediate stress response, catecholamines coincide with the rapid non-genomic glucocorticoid effects, thereby reallocating neuronal resources to the salience network to promote vigilance, encoding of experiences, and stimulus-response behaviour ${ }^{11-13}$. Subsequently, delayed genomic actions of glucocorticoids boost the prefrontal executive control network to restore homeostasis in the aftermath of stress ${ }^{11}$. This time-specific dual mode of action of glucocorticoids in the brain ${ }^{11,14}$ indicates that it is important to investigate the temporal dynamics of the stressinduced shift in brain activity patterns or in interregional neuronal interactions (i.e., functional connectivity). Acute stress-induced changes in functional connectivity can be investigated by repeated resting state assessments. Since the amygdala is one of the fastest brain areas to react to a stressor, we investigated stress-induced changes in functional connectivity of the amygdala at rest [Chapter 2]. In healthy participants, amygdala connectivity was assessed three times using resting state fMRI: at baseline, immediately after acute stress induction (i.e., immediate stress phase), and 30-min after stress induction (i.e., early recovery phase).

The immediate stress phase was defined as the difference between the resting state scan immediately after stress induction and the baseline scan. We found that the amygdala - left ventrolateral PFC and the amygdala - ventral posterior cingulate cortex (PCC) connectivity was reduced immediately after stress. These areas determine the emotional significance of a stimulus and are involved in identifying threat ${ }^{5,7,15}$. Moreover, immediate stress was characterized by a connectivity increase of the amygdala with the right parahippocampal gyrus. The parahippocampal gyrus has a strong functional connection 
with the amygdala ${ }^{16}$ and has been associated with perceived (i.e., subjective) stress ${ }^{17,18}$ Nevertheless, activity in these areas was not modulated by the cortisol response alone, as the specific comparison between responders and non-responders did not reveal a difference in connectivity.

We found that the baseline corrected amygdala - bilateral vmPFC strength was related to individual differences in task-induced cortisol response. In line with the facilitating effect of the ventromedial PFC (vmPFC) on the HPA-axis, cortisol responders were characterized by enhanced baseline corrected amygdala - vmPFC connectivity immediately after acute stress induction (see Figure 1). Our findings extend previous reports of enhanced amygdala - mPFC coupling immediately after acute stress ${ }^{19}$ by directly comparing the intrinsic amygdala connectivity immediately after stress with their connectivity at baseline.

The role of lateralization in the vmPFC still needs to be unravelled. Animal studies demonstrate that the right vmPFC regulates and integrates autonomic and neuroendocrine responses ${ }^{20}$. Moreover, animals showing efficient HPA-axis regulation were characterized by a right lateralized dopaminergic modulation of the $\mathrm{VmPFC}{ }^{21}$. The dopaminergic modulation of the VmPFC prevents excessive HPA-axis activation ${ }^{22}$. Thus, stress-induced vmPFC lateralization and its interaction with dopamine needs to be carefully considered, as this might be a crucial factor in distinguishing those who are vulnerable for psychopathology marked by HPA-axis dysregulation, from those who are not.

\section{Imaging the stressed brain - the early recovery phase}

The early recovery period after the stressor, defined as the change in connectivity between the resting state scans immediately and 30-min after stress induction, was characterized by connectivity changes in areas involved in emotion regulation and appraisal of affective state. The amygdala - VACC connectivity was reduced while the connectivity with the $\mathrm{AACC}$ and bilateral cuneus was enhanced. Interestingly, we found that the amygdala - dACC connectivity was regulated by the amount of iMAST induced glucocorticoid release. Opposite to non-responders, cortisol responders were characterized by reduced amygdala - dACC connectivity during early recovery (see Fig. 1). Our results extend previous reports of enhanced amygdala connectivity with the dACC during acute stress ${ }^{19,26}$ by directly comparing cortisol responders and non-responders, and by directly comparing the connectivity immediately and 30-min after the stressor. Together, these findings suggest a dynamically changing amygdala - dACC connectivity, regulating adaptive behaviour in response to stress.

Interestingly, threat appraisal has been found to moderate the amygdala - dACC $27-32$. A recent imaging study in soldiers found that individual differences in perceived threat during their first deployment moderated the effect of stress on amygdala - dACC connectivity ${ }^{27}$. Stress exposure enhanced the negative coupling in individuals who experienced low levels of perceived threat, but reduced the negative coupling in individuals who experienced high levels of perceived threat. This altered connectivity persisted for 1.5 years after the experience of a severe stressor ${ }^{27-29}$. Thus, the cortisol specific amygdala dACC connectivity change might be related to the appraisal of threat.

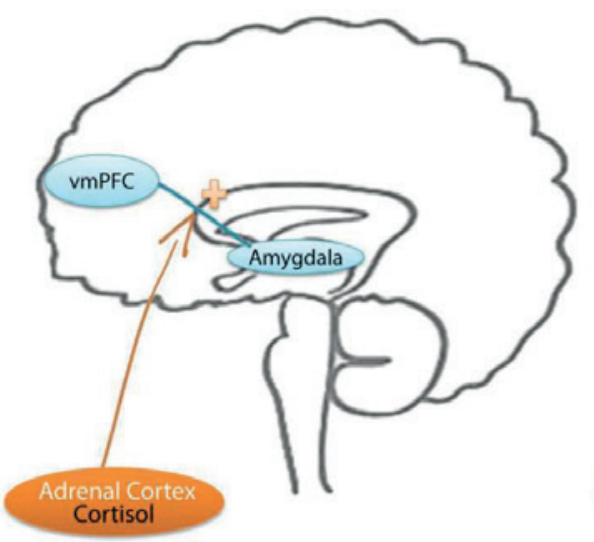

\section{Acute stress}

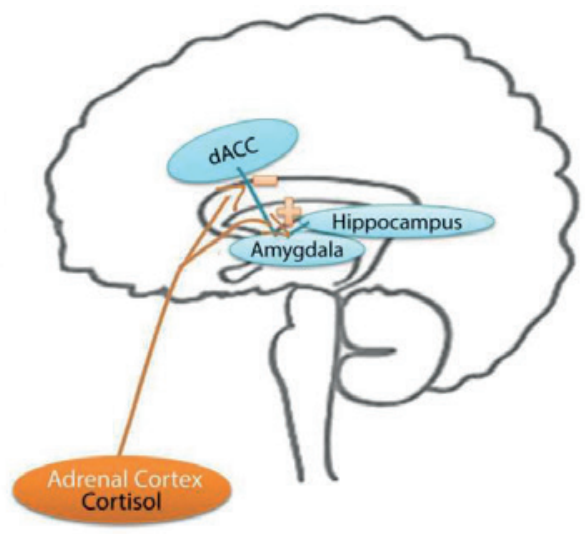

Early Stress Recovery
Figure 1. The phase-specific moderating effect of cortisol on the amygdala connectivity. Cortisol responders were characterized by enhanced amygdala-vmPFC connectivity during the immediate stress phase while during the early recovery phase cortisol responders were characterized by enhanced amygdala-hippocampus and reduced amygdala-dACC connectivity.

\section{Imaging the stressed brain - the early recovery phase \& the hippocampus}

We also found that cortisol moderated the connectivity between the amygdala and anterior hippocampal complex during the early stress recovery phase. Cortisol responders were characterized by an enhanced amygdala - right hippocampal complex connectivity (see Figure 1). The amygdala and hippocampus are thought to interact in response to stress. In a 
prospective study, it was found that the pre-stress amygdala activation was associated with greater symptoms after stress, depending on the degree of hippocampal plasticity following stress $^{33}$. Schwabe and colleagues ${ }^{34}$ found that immediate stress decreased the amygdala hippocampal connectivity during a classification task. This acute stress-induced reduction in functional connectivity correlated with a shift in memory processing from hippocampusdependent declarative toward dorsal striatum-dependent procedural memory ${ }^{34}$. Additionally, a pharmacological fMRI study found that administration of glucocorticoids reduced amygdala - hippocampal resting state connectivity in men ${ }^{35}$. Together, these findings suggest that glucocorticoids boost the amygdala - hippocampal connectivity after an initial reduction following acute stress exposure ${ }^{7}$. This is in accordance with the view that rapid glucocorticoid effects in the amygdala create a brain state that enhances encoding via enhanced attention and alertness while, over time, delayed glucocorticoid effects facilitate the consolidation of memory via suppression of processing of new information and working memory $3,12,13,36,37$. The amygdala - hippocampal connectivity and the interaction of cortisol with the noradrenergic system in the basolateral nucleus of the amygdala (BLA) have both been related to a more pronounced effect of stress on emotional memory consolidation (see Fig. 2) $)^{1,38-42}$.

\section{Imaging the stressed brain - the early recovery phase and the dIPFC}

We found that the immediate stress corrected amygdala - left dIPFC connectivity strength was moderated by the strength of the cortisol responses. Cortisol responders displayed a reduced amygdala - left dIPFC functional connectivity during the early recovery. Thus, our results point to a cortisol-mediated left dIPFC regulatory circuit that serves to adaptively adjust our brain activity to stressors. The modulation of the left dIPFC is probably indirect, since the dIPFC and amygdala are not directly connected ${ }^{43,44}$. Future studies could investigate whole brain network models by for example using independent component analyses to more comprehensively assess how stress affects functional connectivity. The role of lateralized dIPFC activity in stress responding has been further demonstrated in studies using repetitive transcranial magnetic stimulation (rTMS). Activation of the left dIPFC by high frequency rTMS resulted in a lower cortisol response in experimentally stressed women ${ }^{45}$.

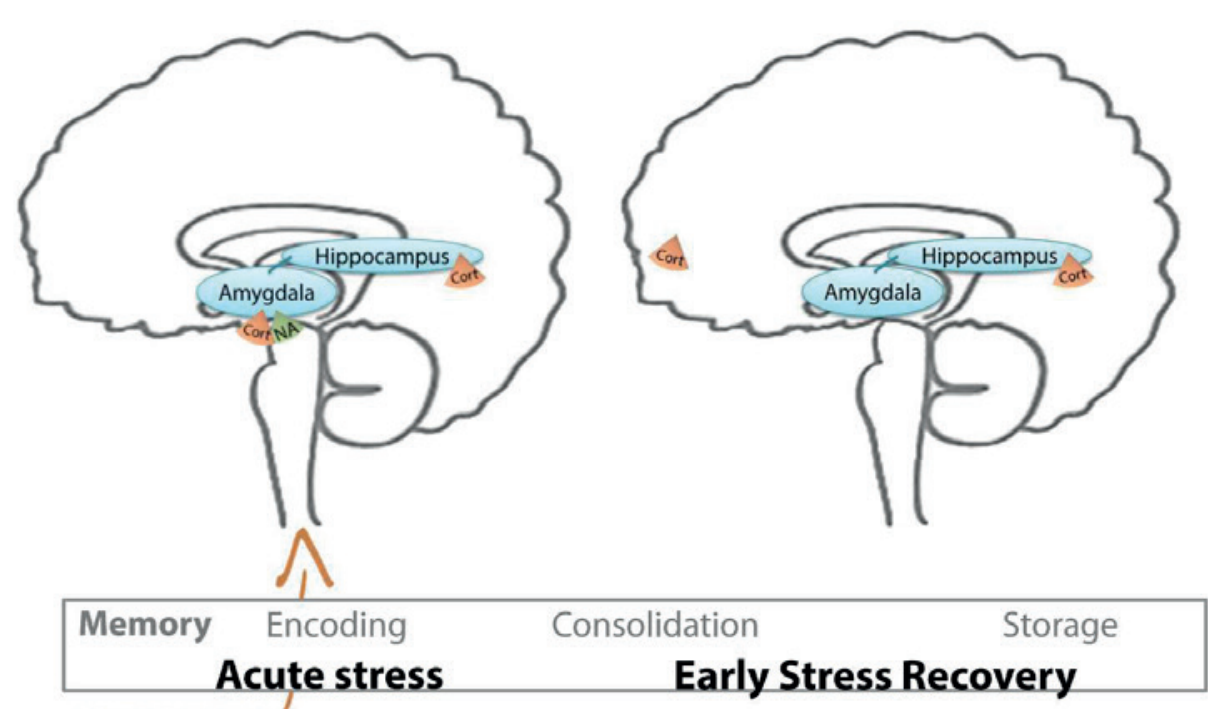

Adrenaline Cortisol

Figure 2. The role of cortisol and noradrenaline during the different phases of stress and memory encoding. Emotionally arousing experiences trigger adrenaline release from the adrenal gland, resulting in the release of noradrenaline (NA) in the BLA. In turn, cortisol is secreted by the adrenal cortex and binds directly bind to receptors in the BLA and to other brain regions. During acute stress, the interaction of cortisol with the noradrenergic system in the BLA enhances memory consolidation by influencing neuroplasticity in other brain regions. Following this period of potential memory enhancement, slow cortisol actions impair the processing of new information via suppression of the hippocampus. We found that during this early recovery phase, cortisol enhanced the connectivity between the amygdala and hippocampus during rest.

Two indirect pathways connecting the dIPFC with the hippocampus and the ACC ${ }^{46}$ were proposed as a mechanism. Increased activity in the dIPFC results in i) enhanced activity in the ACC inhibiting the amygdala, and ii) enhanced activity in the hippocampus. Both reduced amygdala and enhanced hippocampal activity result in a reduced release of corticotrophin-releasing hormone $(\mathrm{CRH})$ from the hypothalamus and, ultimately, decreased cortisol concentrations ${ }^{8,47}$. However, as high frequency rTMS over the right dIPFC has no effect on cortisol levels in an unchallenged situation, the dIPFC asymmetry may only become apparent during high arousing states ${ }^{48}$. Moreover, studies in psychiatric disorders point out the importance of further investigating the role of asymmetric dIPFC activity and 
connectivity in acute stress responding. rTMS induced either an increase in left-sided or a decrease in right-sided dIPFC activity, and both resulted in improvements of depression ${ }^{49,50}$ and anxiety symptoms $\mathrm{s}^{5,52}$.

In sum, we found that cortisol responders displayed a reduced amygdala - left dIPFC functional connectivity during the early recovery phase. We did not assess whether the lateralized connectivity findings are a result of a functional asymmetry, as we did not directly compare it with the activity in the right dIPFC. Future work could investigate functional asymmetry by extracting the data of the same region in both hemispheres and directly testing an interaction effect.

Note that we cannot infer any directionality based on our correlational seed-based connectivity analyses. An interesting next step would be to use a Granger causality approach $^{53}$. Granger causality analyses could be used to investigate whether the stressinduced changes in amygdala connectivity are a result of bottom-up (i.e., the amygdala influencing other regions), or top-down (i.e., other regions influencing the amygdala) processing. Moreover, as spontaneous activity patterns reveal information about the functional organization of the brain ${ }^{54}$, it would be interesting to investigate the relation between time-dependent stress-induced changes in spontaneous functional connectivity as well as in task-dependent recruitment of those same cortical circuits. For example, learning of neutral and arousing material involves two distinct hippocampal connectivity patterns that are also implicated in the time-specific dual mode of action of glucocorticoids in the brain. Specifically, encoding of arousing stimuli has been found to activate the amygdala - hippocampal network, while encoding of neutral stimuli activates the PFC hippocampal network ${ }^{55}$

\section{Imaging the stressed brain - predicting the stress response}

Studying the role of amygdala connectivity in stress responding [Chapter 2], we observed that the intrinsic baseline connectivity between the amygdala and the right dorsal mPFC (dmPFC) predicted variability in the later acute stress induced cortisol response. Specifically, variability in baseline amygdala - right dmPFC connectivity explained $54 \%$ of the variance in cortisol increase in response to the iMAST. Higher amygdala - right dmPFC connectivity prior to stress induction predicted a lower cortisol response. Our baseline results are in line with animal studies suggesting that the right dmPFC inhibits the HPA-axis via modulation of negative feedback ${ }^{56}$. Moreover, these task-absent baseline findings are supportive of the idea that the amygdala is involved in determining the trait response to threat ${ }^{57,58}$

\section{Imaging the stressed brain - inverted U}

It has been suggested that both low and high levels of glucocorticoids can interfere with cognitive functioning and that this is particularly true for processes dependent on the hippocampus ${ }^{12,23,24}$. Hitherto, no neuroimaging studies have investigated a possible inverted U-shaped stress-induced cortisol mechanism in the human brain. Exploratory analyses on previously unpublished data were performed to investigate a possible U-shaped effect of cortisol on the amygdala connectivity during immediate stress. To this end, participants with a cortisol response up to $2.5 \mathrm{nmol} / \mathrm{I}$ were classified as low, between 2.5 and $5.0 \mathrm{nmol} / \mathrm{l}$ as medium, and above $5.0 \mathrm{nmol} / \mathrm{l}$ as high cortisol responders. In medium responders, acute stress enhanced the functional connectivity between the amygdala and cuneus, whereas it reduced the strength in the low and high responder group. The enhanced functional connectivity in medium responders could entail a mechanism of enhanced visual processing that optimizes detection of threats. Of course, a further exploration of this possible U-shaped mechanism is warranted and needed. Animal research has been very valuable regarding the time-dependent involvement of the mineralocorticoid receptor (MR) and glucocorticoid receptor (GR) receptors in stress responses ${ }^{25}$. Likewise, animal research could be the first step to define a possible U-shaped cortisol mechanism on processing in the brain after stress.

\section{Imaging the stressed brain - a network model}

Studies investigating regional differences in emotion processing using task-related contrasts, found a hyper-activation of the amygdala and insula and hypo-activation of the anterior cingulate cortex and prefrontal cortex in posttraumatic stress disorder (PTSD) ${ }^{59,60}$ We found time- and spatial-specific changes in amygdala connectivity with the core areas of the salience network compromising the ventral emotional processing system (e.g., ventral ACC and PFC ${ }^{16,17}$, and the dorsal control network (e.g., hippocampus, dorsal ACC and PFC) ${ }^{17}$. One interesting aspect of our observations is the correspondence between the pattern of acute stress-induced amygdala resting state connectivity changes and the functional 
changes found within the salience and dorsal control network in stress-related psychopathology like PTSD and depression ${ }^{18-23}$.

Although amygdala connectivity provides a valuable framework to study the effects of acute stress, to understand higher cognitive function, we should investigate intrinsic spatial connectivity networks. Alternations in one region cannot be regarded independent from what happens in connected areas. That is, glucocorticoid actions in the amygdala seem to modulate network changes in areas that are reciprocally connected such as the hippocampus and the $\mathrm{PFC}^{3,61,62}$. For example, activation of the amygdala modulates longterm potentiation in the hippocampus and $\mathrm{PFC}^{3,63}$. Moreover, the core affected areas in PTSD are all part of different networks and the functioning of these networks has been related to specific clinical symptoms in PTSD ${ }^{64}$. Specifically, the salience network including the $\mathrm{dACC}$ and insula has been associated with altered threat sensitivity contributing to hyper vigilance and hyper arousal symptoms. The central executive network including the dIPFC has been associated with cognitive dysfunctions, while the default mode network including the MPFC has been associated with an altered meta-awareness. Future studies could investigate interactions between these different intrinsic networks ${ }^{65}$. Moreover, future studies could profit from assessing functional asymmetries in these networks more in-depth. Neuroimaging studies have identified context-dependent lateralisation in specific frontal brain regions during emotional processing ${ }^{66-69}$. Additionally, a time-dependent lateralisation of the amygdala has been suggested, with the right amygdala regulating fast implicit threat detection and the left amygdala regulating slow explicit emotion appraisal processes ${ }^{70}$. Therefore, a hemisphere level analysis of functional differentiation will not provide a desirable level of specificity ${ }^{71}$.

\section{The role of timing of stress on memory formation}

The time-dependent change in brain processing induced by the acute physiological stress response promotes behavioural adaptation in times of stress. Enhanced memory consolidation, especially of emotional experiences ${ }^{72-74}$, is a well-known long-term adaptive behavioural consequence of stress exposure. Much like acute stress does, the emotional arousal that is generated by emotional stimuli boost attentional resources via engagement of subcortical structures that in turn modulate visual processing ${ }^{75,76}$. The effect of stress on emotional memory is regulated by the combined glucocorticoid and noradrenergic activity in the amygdala and is also time dependent ${ }^{13,77,78}$. This time-dependency is strongly rooted in a large body of neuroendocrinological literature demonstrating that stress hormones influence memory performance dependent on the memory phase (i.e., consolidation enhancement ${ }^{79,80}$ or retrieval impairment ${ }^{81-83}$; for an extensive review see $\left.{ }^{84}\right)$. However, this time window is more specific than the consolidation versus retrieval phase. The exact temporal dynamics of stress hormone release relative to the learning that takes place are even crucial within the consolidation memory phase ${ }^{1,13}$. When the release of stress hormones and learning coincide in time, stress initiates a rapid enhancement of hippocampal neuroplasticity via the amygdala. This is followed by an enhancement of hippocampal neuroplasticity via non-genomic membrane MR activity ${ }^{1,46,85}$. When there is a delay between the stress hormone release and hippocampus-dependent learning, genomic GR actions induce a refractory state of the hippocampus, thereby impairing the processing of new information'. Nonetheless, experimental studies testing this stress-timing hypothesis in humans are sparse (e.g., pre-learning ${ }^{86}$; retrieval ${ }^{87}$ ).

The Maastricht Acute Stress Test (MAST) was employed to investigate the effect of timing of a stressor on memory encoding [Chapter 3]. Acute stress was induced either immediately or 30-min before learning emotional stimuli that were taken from the International Affective Picture System (IAPS) ${ }^{88}$. The time-dependent stress effect was assessed at the behavioural and at the electrophysiological level. A delayed memory test was carried out 24 hours later. Event-related potentials (ERPs) were measured with EEG to investigate the underlying temporal mechanism of the stress-timing effect. We found that timing of stress influenced the direction of the association between the increase in cortisol response and the amount of remembered pictures $24 \mathrm{~h}$ later. To be specific: correctly recognized neutral pictures were positively associated with the cortisol response within the immediate stress condition, whereas a negative association within the 30-min pre-learning stress condition was found (i.e., fewer pictures recognized with higher cortisol responses). This behavioural association in the 30-min pre-learning stress condition was supplemented by a negative association between the amplitude of the late positive potential (LPP; an electrophysiological index of attention and visual processing ${ }^{89}$ ) in the 500-1000 ms window and the increase in cortisol response. The combined behavioural and electrophysiological results suggest that an underlying attentional mechanism impaired the learning in the 30min pre-learning stress condition. A recent combined fMRI - EEG study found that the LPP 
is generated by a network of cortical and subcortical areas $^{90}$. The LPP amplitude was correlated with the BOLD activity in the amygdala, VIPFC, MPFC, insula, and occipital cortex $^{90}$. The results of the amygdala connectivity study [Chapter 2] also demonstrated a time-dependent stress modulation of visual and attention areas. Specifically, we found that the amygdala - cuneus connectivity was influenced by the time lag after an acute stressor. The amygdala - cuneus connectivity was less negative during the immediate stress phase, compared to baseline, while it was more negative during the early recovery phase ${ }^{91}$. In sum it seems that stress 30-min before encoding decreased attentional allocation to neutral information, thereby impairing the processing of new information.

Our timing of the delayed condition (i.e., 30-min) is too short to disentangle rapid and slow glucocorticoid effects. From animal and pharmacological studies, it is known that the specific temporal profile of MRs and GRs are responsible for different aspects of stressdependent modulation of memory formation. Blockade of the MR before learning affects memory quality by changing the learning strategy ${ }^{44,92}$, while blockade of the GR affects memory quantity by impairing consolidation ${ }^{92,93}$. The majority of studies investigating the temporal profile of MRs and GRs only administered cortisol, even though this does not capture all aspects of the complex stress response ${ }^{94}$. Future studies may want to combine the timing of the stressor with pharmacological manipulations (e.g., selective MR and GR agonists and antagonists) to investigate the role of non-genomic and genomic MR and GR actions. Moreover, stress is multi-faceted and arousal plays a critical role in the effect on memory formation, which makes evident why future studies may profit from simultaneously manipulating the HPA-axis and the ANS system (e.g., by administering cortisol and yohimbine together; see ${ }^{95}$ ).

\section{Brain asymmetry and stress resilience}

Functional asymmetry of the lateral PFC has been suggested to be relevant to stress adaptation, with the left lateral PFC being involved in effective stress coping ${ }^{56,96}$. Moreover, altered lateralized brain activity has been found in stress-related psychopathology ${ }^{97,98}$ Studying the role of amygdala connectivity during the acute stress response [Chapter 2], we found that subjective stress was inversely associated with the amygdala - left dorsolateral PFC (dIPFC) connectivity immediately after acute stress. In line with the proposed role of down-regulation of negative affect ${ }^{99}$, we found indirect evidence that stronger amygdala - left dIPFC connectivity was associated with less experienced stress. In Part $\mathbf{2}$ of the thesis, we first assessed the functional role of frontal alpha asymmetry in acute stress responding. Hereafter, we explored the possibility to modify cortical brain activity via self-regulation, to provide an account for the role of our brain as potential protective factor in stress resilience.

\section{Brain asymmetry and stress resilience - EEG frontal alpha oscillations}

In Chapter 4, we assessed the functional role of frontal lateralization in stress responding by directly measuring the average difference in low-frequency alpha activity between the left and right frontal areas using EEG. Frontal alpha lateralisation was measured across eight minutes at baseline and following exposure to acute stress induced with the MAST in healthy men and women. The alpha activity was measured from the mid-frontal (F4-F3) locations, suggested to cover the dIPFC ${ }^{100-102}$

Relative left-sided frontal baseline asymmetry resulted in a smaller task-induced cortisol response. This association was specific for the F4-F3 location and was only significant when the individual alpha frequency (IAF) was used. It is well known that there are inter-individual variations in spectral frequencies ${ }^{103,104}$ particularly in alpha peak and bandwidth ${ }^{105-107}$. Moreover, individual differences in alpha peak frequency have been related to interindividual differences in several cognitive functions including perception, attention, and memory ${ }^{108}$. We found that the association between frontal asymmetry and the cortisol response was lacking when frontal asymmetry was based on the standard $8-13 \mathrm{~Hz}$ alpha band. Our standard alpha band results are in accordance to previous studies that also found no association with the stress response ${ }^{109,110}$ 
Baseline frontal asymmetry was not associated with measures of subjective stress. Our results support the capability model of individual differences in frontal asymmetry stating that frontal asymmetry does not correlate with current motivational states under all circumstances ${ }^{111}$. The capability model suggests that frontal asymmetry more likely predicts the vulnerability or propensity to experience particular states when it is measured under motivationally challenging conditions. In other words, frontal asymmetry reflects an individuals' capacity for emotion regulation in situations that demand it. This idea is supported by two recent studies demonstrating that only state-specific frontal asymmetry was associated with emotion regulation capacity ${ }^{112}$ and could differentiate between current depression and healthy controls ${ }^{113}$. Nonetheless, the precise motivational circumstances determining the association between frontal asymmetry and affective responding are not fully understood ${ }^{114}$ and require further study. The EEG results of Chapter 4 are in agreement with our fMRI study [Chapter 2], which showed that subjective stress was inversely associated with amygdala - left dIPFC connectivity immediately after acute stress, but not with the baseline connectivity. Taken together, our results suggest that frontal asymmetry, possibly originating in the dIPFC modulates the cognitive processing of threatening information during high arousing states.

Positive and negative emotions generally involve approach versus avoidance motivations, which have been associated with the left and right prefrontal cortex ${ }^{115}$. These approach and withdrawal systems could be related to differences in resilience, more specifically by how these differences bias motivational processing ${ }^{116}$. We found that relative left-sided frontal asymmetry was related to more behavioural activation. This, together with our finding that relative left-sided frontal asymmetry resulted in a smaller cortisol response, argues in favour of the idea that stress coping might be facilitated via a glucocorticoidmediated prioritization of automatic approach-avoidance responses ${ }^{117}$. Whether stress ultimately facilitates more approach or avoidance related behaviours, though likely depends on the context and the individual. Stress is a motivationally complex concept. For instance, the motivational direction model of frontal asymmetry ${ }^{118}$ would predict fightrelated responses to be associated with approach strategies and left-sided activity. Flight responses, on the other hand, may be driven by withdrawal strategies through fear or approach strategies (i.e., active avoidance), both driven by right-sided activity. Thus, individual differences in the experience of stress in any given situation may be differentially associated with frontal asymmetry. Combined with the finding that approach and withdrawal tendencies transiently suppress each other ${ }^{119}$, this calls for a refinement of the temporal specificity of the measurement of frontal asymmetry. One potential solution would be to measure short bursts of alpha activity instead of averaging across several minutes ${ }^{111}$. Moreover, future studies could focus on measuring frontal asymmetry and affect during the stress task in order to capture its role under motivationally challenging conditions.

The weak strength of the association found between frontal baseline asymmetry and the cortisol response in combination with diverse findings when the alpha band was defined differently, seems to suggest that alpha is a nonspecific asymmetry metric. This together with more general methodological differences in the frontal lateralization literature (e.g., use of reference, length of measurement), questions the foundation of the currently accepted theory about the role frontal lateralization in emotion. Emotions elicited by a stressor are too complex to be understood by simple frontal lateralisation. The findings described in this thesis suggest a need to for refine frontal asymmetry theories by including state-dependency as well as switching from lateralization to a network account. Combined EEG-fMRI studies have demonstrated that phase synchronisation of low-frequency oscillations coordinate communication between distant areas in the brain that contribute to resting-state networks ${ }^{120,121}$. In addition, cross-frequency phase coupling between alpha and high-frequency oscillations may play a significant role in cognitive processes ${ }^{121}$. Studies are needed that assess frontal asymmetry in the alpha band in relation to synchronisation in other frequency bands during stress in healthy participants and in patient populations.

\section{Brain asymmetry and stress resilience - modification of cortical brain activity}

The results from Chapters $\mathbf{2}$ and $\mathbf{4}$ suggest that the left dIPFC might be a target area for brain activity based treatments to enhance stress resilience. We explored the validity of EEG neurofeedback to modulate relative asymmetry of the frontal cortex (i.e., F4-F3 covering the dIPFC) [Chapter 5]. While it has been often reported that people can learn to change their own brain activity via neurofeedback, this has rarely been investigated appropriately. To reiterate, our experiment consisted of a baseline test day assessing relative frontal asymmetry and acute stress responding, followed by 6 neurofeedback sessions and two follow-up test days (i.e., one week and one month later) again assessing relative frontal asymmetry and 
acute stress responding. In particular, we compared three frontal asymmetry neurofeedback protocols that were developed to increase relative right-sided frontal alpha asymmetry, to increase relative left-sided frontal asymmetry, or to yield no effects on frontal alpha asymmetry (i.e., the placebo control group). Participants in the placebo condition were not fed back their own current asymmetry, but instead their feedback was based on training scores of ten participants from a pilot study. We hypothesised that following six days of neurofeedback, participants trained to shift relative frontal activity towards the left hemisphere (i.e., relative right-sided frontal alpha power) were expected to show decreased negative affect and decreased subjective and neuroendocrine stress responses. The opposite pattern of findings was expected for the right group. No changes over time were hypothesized for the placebo group.

Individual frontal alpha frequency neurofeedback resulted only in a change in relative frontal asymmetry at rest in participants in the right-trained direction. The learning occurred independently of other frequency bands and locations, but did not persist one week and one month follow-up. The change in relative asymmetry to the right was only found during the rest measurement and not during the feedback itself. This could be due to the fact that learning from feedback involves high levels of cognitive effort and outward attention. Importantly, both cognitive effort and outward attention are accompanied by alpha suppression, which may decrease the sensitivity to detect asymmetry changes. Furthermore, it is possible that neurofeedback is more effective in changing tonic rather than phasic alpha asymmetry as tonic alpha changes occur at a slower rate ${ }^{122}$. It has been shown that manipulation of alpha oscillations during rest is related to high performance levels and alpha suppression during cognitive tasks ${ }^{123}$.

On the behavioural level, we found that the change in relative frontal alpha asymmetry in the right group seemed to preclude a decrease in subjective stress on test day 2. These results fit well with the association found in Chapter $\mathbf{2}$ during immediate stress between the amygdala - left dIPFC connectivity and reduced task-induced subjective stress. No changes in unprovoked current mood were observed over the six training days. This latter finding is in line with previous frontal asymmetry studies ${ }^{124,125}$, with the baseline frontal asymmetry findings of Chapter $\mathbf{4}$ as well as with the baseline amygdala - left dIPFC connectivity findings of Chapter 2. The neurofeedback training direction did not differentially change the task-induced cortisol response between test days 1 and 2 . This corroborates the findings of Chapter 4 showing no stress-induced changes in frontal asymmetry. A recent rTMS study found that in an unchallenged situation, a change in relative frontal activity had no effect on cortisol levels $\mathrm{s}^{38}$, while the same procedure did change the cortisol response in experimentally stressed healthy participants ${ }^{35}$. These rTMS findings agree well with our results and suggest that the moderating role of lateralised frontal activity on the fight-or-flight response is state-dependent.

There is a need for studies investigating whether neurofeedback may be useful in combination with behavioural interventions to open a window of opportunity that may enhance the efficacy of the other treatments. In general, to assess the effectiveness and behavioural usefulness of neurofeedback training, studies should compare neurofeedback with other therapies such as cognitive behavioural therapy, pharmacological treatment or the combination. Importantly, our results indicate that the change in frontal asymmetry did not persist over time and that there are marked individual differences in the ability to learn how to self-regulate frontal asymmetry. Only $44 \%$ of the participants trained in the left direction and $75 \%$ trained in the right direction demonstrated a change in asymmetry. These limited findings make us question whether effects are universally beneficial, and also suggest that it is highly unlikely that EEG neurofeedback can be used as an effective standalone treatment.

\section{Behind the scenes - methodological consideration}

In this thesis, the contribution of different brain areas in post-stress regulation was investigated to identify what makes people thrive in the face of adverse life events. Moreover, to further explore the role of glucocorticoids in stress resilience, we tested the time-dependent effects of acute stress on brain connectivity and memory formation. Finally, we tried to determine to what extent frontal brain asymmetry is functionally relevant for stress responding and went beyond the limits of correlational studies by using neurofeedback. To accomplish this, we made specific choices with regard to the study population, experimental manipulation, and the design of the study. With a view towards the future, we will discuss some general methodological considerations. 


\section{Study population}

We included healthy young participants in all studies. The advantage of working with a healthy population for acute stress research lies precisely in the fact that these subjects have not yet been exposed to any major stressful experiences. This enables investigating mechanisms that could contribute to the development of psychopathology. Moreover, we kept age consistent (i.e., between 18 and 35 years) since it is known that aging influences the effects of glucocorticoids on memory ${ }^{126,127}$. However, as a consequence, the generalization of our results to other populations remains to be empirical tested.

Furthermore, gender is an important biological determinant of the effects of stress on cognition and brain function ${ }^{128,129}$. It has been suggested that men and women use different stress coping strategies ${ }^{130}$. This might be due to differences in engagement of frontal and limbic structures during stress processing. For instance, using the serial subtraction task to induce acute stress, Wang and colleagues ${ }^{131}$ found that stress activated the right prefrontal cortex in men, whereas in women stress activated the ventral striatum, putamen, insula, and cingulate cortex. Notably, these differences were observed in the absence of a difference in the physiological stress response. Wang and colleagues ${ }^{131}$ also showed that after completion of the serial subtraction task, only women displayed activity in the anterior and posterior cingulate cortex, structures that have been implicated in emotional processing and reflection of emotional traits. Besides, it is known that the menstrual cycle affects the stress response in women ${ }^{132}$. Particularly, the menstrual cycle was found to modulate activation of subcortical arousal structures that are part of stress response circuitry ${ }^{133,134}$. These findings suggest that gender should be taken into account when investigating the contribution of different brain areas in post-stress regulation. Meanwhile, many neuroimaging studies have included only men or did not address gender differences in the study design due to relatively small sample sizes. In the studies described in Chapter 1, 2, 4, and 5, both men and women were included, but no gender differences were found. One reason for our finding that gender did not play major role could lie in the fact that women were included only if they used oral contraceptives to avoid menstrual cycle phase-dependent cortisol differences. Additionally, the use of the bilateral amygdala as seed in the fMRI study could also have masked a gender difference, as gender-related differences in amygdala connectivity during rest have been demonstrated with stronger right amygdala connectivity in men and stronger left amygdala connectivity in women ${ }^{135}$
Including gender as an explicit to-be-tested factor in the design of future studies (e.g., by including women during the various phases of the menstrual cycle), and specifically evaluating the laterality of the amygdala, could aid tremendously in improving our understanding of specific gender related vulnerability patterns for stress-related disorders.

\section{Stress induction}

The definition of acute stress is broad and the physiological response to acute stress is multidimensional. It is known that, in the lab, psychological measures (i.e., questionnaires) of stress often disagree with physiological ones like heart rate, blood pressure or cortisol. This suggests that there is no single best measure of determining acute stress in the lab. In our view, what it means to be stressed is, at least in part, showing a physiological stress response. Consequently, in this thesis stress was mainly evaluated based on the HPA-axis reactivity (e.g., cortisol responses). Salivary cortisol is related to stress-induced changes in behavioural outcomes, indicating that it is linked to affective and cognitive processes regulated by our brain. Moreover, using cortisol as a measure of stress is clinically relevant. There is substantial evidence of an altered functioning of the neuroendocrine system in depression (i.e., distorted basal cortisol levels) and PTSD (i.e., lowered basal cortisol levels). Studies investigating HPA-axis reactivity in PTSD have yielded divergent findings. The studies that use laboratory stressors report lower task-induced cortisol responses ${ }^{136-139}$, while studies using trauma reminders ${ }^{140}$ or anticipation of stressful events ${ }^{141-143}$ report elevated cortisol responses. This raises an important question for future research: Do different stressors converge on common resilience mechanisms?

We only investigated the effect of a single acute stressor on processing in the brain. Animal research has shown that prolonged periods of stress affect both brain function and structure in a region-specific manner. Specifically, there is a functional distinction between the effects of prolonged stress hormone exposure on the dorsal cognitive control regions (i.e., reduced hippocampus and mPFC volume and function) ${ }^{144-146}$, on the one hand, and the ventral emotion processing regions (i.e., enhanced amygdala volume and function) ${ }^{144}$, on the other hand. Besides, the effect of stress on the amygdala may also depend on previous stressful experiences ${ }^{46}$. Since the effect of a single acute stressor might well be different from prolonged exposure, the generalization of our results to chronic stress effects on the brain is quite limited. 
Acute stress was induced using the (i)MAST and, in Chapter 5, we demonstrate that this laboratory stressor can be used repeatedly: no differences in cortisol response between the three stress days were found in the placebo neurofeedback condition. Importantly, the neurofeedback study described in Chapter $\mathbf{5}$ and the fMRI study described in Chapter $\mathbf{1}$ and 2 only included a stress condition, but not a no-stress control condition. For future research, a no-stress placebo version of the iMAST could be very practical. Consistent with the placebo version for the MAST ${ }^{147}$, the iMAST' $s$ no-stress placebo version could consist of small, relatively neutral (e.g., $35^{\circ} \mathrm{C}$ and $40^{\circ} \mathrm{C}$ ) temperature deviations, and a simplified counting task (counting consecutively from 1 to 25 ) without any performance feedback being given to the subjects. This way, the no-stress placebo version would be similar to the IMAST in terms of physical and mental load as well as in its duration, but without eliciting stress reactions. Such a placebo condition will allow a better evaluation of the effects of stress on various cognitive tasks more reliably.

\section{Divergent opinions on how to define a neurofeedback responder}

Generally speaking, there is not a single laboratory stress task that is capable of inducing a significant physiological stress response in all participants. The same principle seems to hold for neurofeedback in that not all participants are able to learn how to modulate their cortical activity using EEG neurofeedback ${ }^{122,148,149}$. Previous neurofeedback studies have used different responder classification criteria and did not assess the validity of the employed classification on an independent measure. We defined responders as participants who produced a significant shift in the trained direction in relative frontal asymmetry (i.e., difference between training 6 and training 1 ) as assessed during baseline. The validity of the classification was tested on the trainability during neurofeedback. We found that the baseline classification differentiated responders from non-responders during the feedback training itself, whilst no effect of the neurofeedback training was found for the whole group analysis. Moreover, individual differences in trainability were dependent on the training direction, with participants in the right direction being more likely to change their frontal asymmetry in the desired direction, relative to participants in the left direction condition. This finding underscores the importance of making a responder versus non-responder distinction to accurately evaluate the effectiveness of neurofeedback protocols in terms of the magnitude of the trained responses within such responders groups. Clearly defining a training responder criterion is also important for therapeutic reasons as this would enable therapists to determine the end point of the training, or to identify those participants who do not respond to neurofeedback treatment as early as possible.

\section{The neurofeedback protoco}

In order to optimize learning, we developed and used a novel frontal asymmetry neurofeedback protocol. Our neurofeedback protocol calculated real-time averages of both references and corrected eye blinks online. Eye movement artefacts influence the EEG activity, especially at the frontal sites and in the alpha band. It is therefore said to be important to correct eye blinks real-time when using a frontal alpha asymmetry neurofeedback training protocol ${ }^{150,151}$. Also, a computerized linked mastoids (i.e., average of $A 1+A 2)$ reference has a superior signal-to-noise ratio compared to the previously used $C z$ reference ${ }^{152}$. Individual alpha was used because there are large individual differences in alpha ${ }^{114,115}$ and using the conventional frequency band may impair the trainability of frontal asymmetry. Nonetheless, not all participants learned how to modulate their cortical activity. Our neurofeedback protocol was based on frequency as information source. Note that a frequency represents spatio-temporal overlap of many simultaneously active brain systems and this might be too general to learn how to change frontal brain asymmetry. Increasing the specificity of the feedback signal might result in an increase of the efficiency. This could be done for example by using multi-channel systems to include spatial information (e.g., low-resolution electromagnetic tomography; LORETA) or by including interactions between different brain regions using coherence or phase synchrony. Alternatively, the feedback could be based on event-related potentials instead of alpha power. The late positive potential (LPP; see Chapter 3) is in this regard interesting, as the early LPP is sensitive to intrinsic factors (e.g., emotionality of the stimuli) while the late LPP reflects regulation of stimuli153-155. Moreover, the late LPP has been used as neural marker of approach and avoidance actions to emotional stimuli ${ }^{156}$. Event-related neurofeedback (i.e., targeting the LPP) is more specific on the neural level (i.e., LPP) and includes a specific event (i.e., emotional stimuli). Hence, this would go beyond EEG power activity related to broad brain areas, and might be more effective.

Providing participants with a strategy might be another way to increase efficiency. Real time fMRI (rt-fMRI) neurofeedback ${ }^{157}$ is effective and is often performed using active 
tasks like (instructed) imagination ${ }^{158,159}$ and emotion regulation strategies. For example, Johnston and colleagues ${ }^{159}$ trained participants to upregulate the insula and amygdala using self-generated mental images. They found that participants were able to learn to upregulate the emotion areas after a brief training. Moreover, the activation increases were not confined to the target areas, and included areas related to emotional memory and imagination like the left dmPFC, ACC, striatum, basal forebrain, and parietal cortex ${ }^{159}$ Sarkheil and colleagues ${ }^{160}$ instructed participants to reappraise evoked negative emotions while feedback of left PFC activity was provided by rt-fMRI. They found that the fMRI feedback resulted in a reduced amygdala response during emotion regulation of aversive stimuli. Brühl and colleagues ${ }^{161}$ used reality checking as emotion regulation strategy to successfully down-regulate amygdala activity while viewing negative emotional faces in four rt-fMRI sessions. A possible strategy to change relative frontal asymmetry could be motor imagery. Motor imagery modulates the sensorimotor-rhythm ${ }^{162}$, which is linked to the alpha frequency. Moreover, both frequencies are suggested to be regulated by the thalamocortical system ${ }^{121,163}$. Participants could be instructed to approach positive stimuli by imagining to walk to the stimuli or to avoid negative stimuli by walking away from the stimuli to increase relative left and right-sided frontal asymmetry, respectively. Such an active task, however, will likely rely on a different mechanism of regulation. The only study that investigated strategy use for frontal asymmetry training found no differences in strategies employed between an increase and decrease left frontal asymmetry group ${ }^{164}$ These results cast doubt on whether frontal alpha asymmetry could be regulated by an explicit strategy. To optimize the efficacy of neurofeedback training, future studies could evaluate strategy use in frontal asymmetry neurofeedback. In general, more research is needed into the specific parameters like the duration of a feedback block, the modality and design of the feedback, and the design of the control condition. Besides, future studies could include fMRI measurements before and after the neurofeedback training to assess the underlying mechanism and to gain knowledge about the neural networks modulated by successful EEG neurofeedback. A recent study showed that a 30-min EEG neurofeedback training targeted at suppression of alpha amplitude changed the functional connectivity of the $\mathrm{dACC}$ within the salience network ${ }^{165}$. Interestingly, the degree of alpha reduction during EEG neurofeedback was predictive of the resting-state alpha amplitude change measured with EEG, which was negatively correlated with the enhanced connectivity in the salience network. Finally, future studies could assess transfer and generalisation from the laboratory setting to real life. In our study, transfer was assessed at the very end of the last neurofeedback session by asking the participants to try to produce the same activity as during learning but now while watching a black screen and getting no feedback to see whether they could produce the activity without the feedback. We are currently evaluating such transfer in the right trained group.

\section{fMRI study design}

Based on the findings of a recent study showing that changes in salience network connectivity after exposure to a fear-related stressor (i.e., a movie fragment depicting extreme violence) are partly driven by noradrenergic signaling ${ }^{166}$, we used a time window of 30-min after the end of the acute stress induction phase, because in that interval both autonomic and glucocorticoid stress mediators are still substantially elevated. However, using a 30-min time period has as consequence that the reported early recovery effects are most likely the result of non-genomic glucocorticoid effects on the amygdala's connectivity. The exact role of rapid non-genomic and delayed genomic glucocorticoid actions (i.e., glucocorticoids' dual mode of action) on the functional amygdala network in humans is limited. Two elegantly designed pharmacological fMRI studies investigated the timedependent non-genomic and genomic effects on amygdala connectivity during selective attention ${ }^{167}$, and vigilance regulation ${ }^{168}$. Slow genomic glucocorticoid effects reduced the amygdala - cuneus coupling during selective attention ${ }^{167}$ while it enhanced the amygdala mPFC coupling accompanied by higher amygdala activity for negative compared to positive faces ${ }^{168}$. It would be interesting to also investigate the delayed genomic effect of acute stress-induced cortisol on changes in amygdala connectivity by repeatedly measuring resting state measurements during a longer period after the stress induction. Moreover, pharmacological manipulations could be combined with an acute stress exposure to disentangle the early and delayed effects.

Physiological responses, such as cardiac or respiratory rate, might have a confounding effect on resting-state measurements ${ }^{169,170}$. Particularly, correction is important when a data driven approach is used (see Chapter $\mathbf{2}$ of this thesis). We aimed to minimize these possible confounding effects by removing signals from ventricular and white matter areas and by restricting the resting state functional connectivity analyses to low frequencies. 
Moreover, we used a within-subjects design (i.e., constructing differences scores) to correct for possible stress-unrelated effects. Still, the amygdala activity might have been generally higher during stress as compared to the baseline. Future studies investigating stress processing in the brain could measure the effects of acute stress on perfusion patterns of the brain for example with arterial spin labelling ${ }^{171}$.

\section{Concluding Remarks}

Stress resilience may be revealed by a combination of adaptive neural responsiveness during acute stress and its recovery, in combination with vulnerability or resilience factors. Pertaining to the stress vulnerability models that were discussed at the beginning of this dissertation, personality traits, early life experiences, and the genetic make-up will influence brain function and, thereby, how someone will cope with stressful situations. For instance, personality traits like neuroticism have been related to individual differences in activity of the amygdala in response to emotional stimuli ${ }^{172}$. Furthermore, variations in genes coding for the glucocorticoid receptors have been related to emotional memory processing ${ }^{173,174}$, cortisol responsiveness to stress ${ }^{175,176}$, and the susceptibility to develop PTSD ${ }^{177}$ and depression ${ }^{178}$. Moreover, different early stress experiences result in either a HPA-axis hyperor hypo-function, due to a different regulation of glucocorticoid receptors in the hippocampus ${ }^{179-181}$. In turn, altered HPA-axis activity shapes the activity in prefrontal areas as well as the connectivity between prefrontal areas and the amygdala, which in turn influences the processing of emotions and stress coping strategies ${ }^{179,182}$. It is our hope that by continuing to learn from imaging the brain, stress vulnerability models can be further refined to ultimately develop specific models that are capable of describing and predicting when and how people will be resilient in the face of adversity.

\section{References}

1 Joels, M., Fernandez, G. \& Roozendaal, B. (2011). Stress and emotional memory: a matter of timing. Trends in Cognitive Sciences, 15, 280-288.

2 Krugers, H.J., Karst, H. \& Joels, M. (2012) Interactions between noradrenaline and corticosteroids in the brain: from electrical activity to cognitive performance. Frontiers in Cellular Neuroscience, 6.

3 Roozendaal, B., McEwen, B.S. \& Chattarji, S. (2009). Stress, memory and the amygdala. Nature Reviews: Neuroscience, 10, 423-433.

4 Miller, R., Plessow, F., Kirschbaum, C. \& Stalder, T. (2013). Classification Criteria for Distinguishing Cortisol Responders From Nonresponders to Psychosocia Stress: Evaluation of Salivary Cortiso Pulse Detection in Panel Designs. Psychosomatic Medicine, 75, 832-840.

5 Dedovic, K. et al. (2009). Neural correlates of processing stressful information: An event-related fMRI study. Brain Research 1293, 49-60

6 Khalili-Mahani, N., Dedovic, K., Engert, V. Pruessner, M. \& Pruessner, J. C. (2010). Hippocampal Activation During a Cognitive Task Is Associated With Subsequent Neuroendocrine and Cognitive Responses to Psychological Stress. Hippocampus, 20, 323-334.

7 Pruessner, J.C. et al. (2008). Deactivation of the limbic system during acute psychosocial stress: evidence from positron emission tomography and functional magnetic resonance imaging studies. Biological Psychiatry, 63, 234-240.

8 Herman, J.P. et al. (2003). Centra mechanisms of stress integration hierarchical circuitry controlling hypothalamo-pituitary-adrenocortical responsiveness. Frontiers in Neuroendocrinology, 24, 151-180.
9 Ulrich-Lai, Y.M. \& Herman, J.P. (2009) Neural regulation of endocrine and autonomic stress responses. Nature Reviews: Neuroscience, 10, 397-409.

10 Dedovic, K. et al. (2005). The Montreal Imaging Stress Task: using functional maging to investigate the effects of perceiving and processing psychosocial stress in the human brain. Journal of Psychiatry and Neuroscience, 30, 319-325.

11 Hermans, E.J., Henckens, M.J.A.G., Joels, M. \& Fernandez, G. (2014). Dynamic adaptation of large-scale brain network in response to acute stressors. Trends in Neurosciences, 304-314.

12 Joels, M., Pu, Z., Wiegert, O., Oitzl, M. S. \& Krugers, H.J. (2006). Learning under stress: how does it work? Trends in Cognitive Sciences, 10, 152-158.

13 Diamond, D.M., Campbell, A.M., Park, C.R Halonen, J. \& Zoladz, P.R. (2007). The temporal dynamics model of emotiona memory processing: a synthesis on the neurobiological basis of stress-induced amnesia, flashbulb and traumatic memories, and the Yerkes-Dodson law. Neural Plasticity, 2007, 60803.

14 Joels, M., Karst, H., DeRijk, R. \& de Kloet E.R. (2008). The coming out of the brain mineralocorticoid receptor. Trends in Neurosciences, 31, 1-7.

15 Phillips, M.L., Drevets, W.C., Rauch, S. L. \& Lane, R. (2003). Neurobiology of emotion perception I: The neural basis of norma emotion perception. Biological Psychiatry, 54, 504-514.

16 Stein, J.L. et al. (2007). A validated network of effective amygdala connectivity. Neuroimage, 36, 736-745.

17 Phillips, M., Ladouceur, C. \& Drevets, W. (2008). A neural model of voluntary and automatic emotion regulation: implications for understanding the pathophysiology and neuro- 
development of bipolar disorder. Molecular Psychiatry, 13, 833-857.

18 Li, H.J. et al. (2014). Examining brain structures associated with perceived stress in a large sample of young adults via voxel-based morphometry. Neuroimage, 92, 1-7.

19 van Marle, H.J., Hermans, E.J., Qin, S. \& Fernandez, G. (2010). Enhanced restingstate connectivity of amygdala in the immediate aftermath of acute psychological stress. Neuroimage, 53, 348-354.

20 Sullivan, R.M. \& Gratton, A. (1999). Lateralized effects of medial prefrontal cortex lesions on neuroendocrine and autonomic stress responses in rats. Journal of Neuroscience, 19, 2834-2840.

21 Sullivan, R.M. \& Dufresne, M.M. (2006). Mesocortical dopamine and HPA axis regulation: Role of laterality and early environment. Brain Research, 1076, 49-59.

22 Sullivan, R.M. \& Gratton, A. (1998). Relationships between stress-induced increases in medial prefrontal cortical dopamine and plasma corticosterone levels in rats: Role of cerebral laterality. Neuroscience, 83, 81-91.

23 Lupien, S.J., Maheu, F., Tu, M., Fiocco, A. \& Schramek, T.E. (2007). The effects of stress and stress hormones on human cognition: Implications for the field of brain and cognition. Brain and Cognition, 65, 209-237.

24 de Kloet, E.R., Oitzl, M.S. \& Joels, M. (1999). Stress and cognition: are corticosteroids good or bad guys? Trends in Neurosciences, 22, 422-426.

25 de Kloet, E.R., Karst, H. \& Joels, M. (2008). Corticosteroid hormones in the central stress response: quick-and-slow. Frontiers in Neuroendocrinology, 29, 268-272.

26 Clewett, D., Schoeke, A. \& Mather, M. (2013). Amygdala functional connectivity is reduced after the cold pressor task.
Cognitive Affective \& Behavioural Neuroscience, 13, 501-518.

27 van Wingen, G.A., Geuze, E., Vermetten, E. \& Fernandez, G. (2011). Perceived threat predicts the neural sequelae of combat stress. Molecular Psychiatry, 16, 664-671.

28 van Wingen, G.A., Geuze, E., Vermetten, E. \& Fernandez, G. (2012). The neura consequences of combat stress: longterm follow-up. Molecular Psychiatry, 17, 116-118.

29 van Wingen, G.A. et al. (2012). Persistent and reversible consequences of combat stress on the mesofrontal circuit and cognition. Proceedings of the National Academy of Sciences of the United States of America, 109, 15508-15513.

30 Bush, G., Luu, P. \& Posner, M.I. (2000). Cognitive and emotional influences in anterior cingulate cortex. Trends in Cognitive Sciences, 4, 215-222.

31 McRae, K., Reiman, E.M., Fort, C.L., Chen, K. \& Lane, R.D. (2008). Association between trait emotional awareness and dorsal anterior cingulate activity during emotion is arousal-dependent. Neuroimage, 41, 648-655.

32 Seeley, W.W. et al. (2007). Dissociable intrinsic connectivity networks for salience processing and executive control. Journal of Neuroscience, 27, 23492356.

33 Admon, R. et al. (2009). Human vulnerability to stress depends on amygdala's predisposition and hippocampal plasticity. Proceedings of the National Academy of Sciences of the United States of America, 106, 14120-14125.

34 Schwabe, L., Tegenthoff, M., Hoffken, O. \& Wolf, O.T. (2013). Mineralocorticoid receptor blockade prevents stressinduced modulation of multiple memory systems in the human brain. Biological Psychiatry, 74, 801-808.

35 Henckens, M.J.A.G., van Wingen, G.A. Joels, M. \& Fernandez, G. (2012).
Corticosteroid Induced Decoupling of the Amygdala in Men. Cerebral Cortex, 22, 2336-2345.

36 Karst, H., Berger, S., Erdmann, G., Schutz, G. \& Joels, M. (2010). Metaplasticity of amygdalar responses to the stress hormone corticosterone. Proceedings of the National Academy of Sciences of the United States of America, 107, 1444914454.

37 Henckens, M.J.A.G. et al. (2012). Dynamically changing effects of corticosteroids on human hippocampal and prefrontal processing. Human Brain Mapping, 33, 2885-2897.

38 LeDoux, J.E. (2000). Emotion circuits in the brain. Annual Review of Neuroscience, 23, 155-184.

39 McGaugh, J.L. (2000). Stress hormones and amygdala regulate long-term memory storage. International Journal of Psychology, 35, 405-405.

40 McGaugh, J.L., McIntyre, C.K. \& Power, A.E. (2002). Amygdala modulation of memory consolidation: interaction with other brain systems. Neurobiology of Learning and Memory, 78, 539-552.

41 Roozendaal, B., Okuda, S., De Quervain D.J.F. \& McGaligh, J.L. (2006) Glucocorticoids interact with emotioninduced noradrenergic activation in influencing different memory functions. Neuroscience, 138, 901-910.

42 Roozendaal, B. (2003). Systems mediating acute glucocorticoid effects on memory consolidation and retrieval. Progress in Neuro-Psychopharmacology and Biological Psychiatry, 27, 1213-1223.

43 Price, J.L. (2005). Free will versus survival: Brain systems that underlie intrinsic constraints on behaviour. Journal of Comparative Neurology, 493, 132-139.

44 Urry, H.L. et al. (2006). Amygdala and ventromedial prefrontal cortex are inversely coupled during regulation of negative affect and predict the diurnal pattern of cortisol secretion among older adults. Journal of Neuroscience, 26, 4415 4425.

45 Baeken, C. et al. (2014). One left dorsolateral prefrontal cortical HF-rTMS session attenuates HPA-system sensitivity to critical feedback in healthy females. Neuropsychologia, 57, 112-121.

46 Ray, R.D. \& Zald, D.H. (2012). Anatomica insights into the interaction of emotion and cognition in the prefrontal cortex Neuroscience and Biobehavioural Reviews, 36, 479-501.

47 Dedovic, K., Duchesne, A., Andrews, J. Engert, V. \& Pruessner, J.C. (2009). The brain and the stress axis: the neural correlates of cortisol regulation in response to stress. Neuroimage, 47, 864 871.

48 Baeken, C., Vanderhasselt, M.A. \& De Raedt, R. (2011). Baseline 'state anxiety' influences HPA-axis sensitivity to one sham-controlled HF-rTMS session applied to the right dorsolatera prefrontal cortex. Psychoneuroendocrinology, 36, 60-67.

49 Chen, J.J. et al. (2013). Left versus right repetitive transcranial magnetic stimulation in treating major depression A meta-analysis of randomised controlled trials. Psychiatry Research, 210, 1260-1264.

50 Loo, C. K. \& Mitchell, P.B. (2005). A review of the efficacy of transcranial magnetic stimulation (TMS) treatment for depression, and current and future strategies to optimize efficacy. Journal of Affective Disorders, 88, 255-267.

51 Osuch, E.A. et al. (2009). Repetitive TMS combined with exposure therapy for PTSD: A preliminary study. Journal of Anxiety Disorders, 23, 54-59.

52 Pallanti, S. \& Bernardi, S. (2009). Neurobiology of repeated transcranial magnetic stimulation in the treatment of anxiety: A critical review. International 
Clinical Psychopharmacology, 24, 163173.

53 Roebroeck, A., Formisano, E. \& Goebel, R. (2005). Mapping directed influence over the brain using Granger causality and fMRI. Neuroimage, 25, 230-242.

54 Fox, M.D., Snyder, A.Z., Vincent, J.L. \& Raichle, M.E. (2007). Intrinsic fluctuations within cortical systems account for intertrial variability in human behaviour. Neuron, 56, 171-184.

55 Kensinger, E.A. \& Corkin, S. (2004). Two routes to emotional memory: Distinct neural processes for valence and arousal. Proceedings of the National Academy of Sciences of the United States of America, 101, 3310-3315.

56 Sullivan, R.M. \& Gratton, A. (2002). Prefrontal cortical regulation of hypothalamic-pituitary-adrenal function in the rat and implications for psychopathology: side matters. Psychoneuroendocrinology, 27, 99-114.

57 Etkin, A. et al. (2004). Individual differences in trait anxiety predict the response of the basolateral amygdala to unconsciously processed fearful faces. Neuron, 44, 1043-1055.

58 Hariri, A.R. et al. (2002). Serotonin transporter genetic variation and the response of the human amygdala. Science, 297, 400-403.

59 Vermetten, E. \& Bremner, J.D. (2002). Circuits and systems in stress. II. Applications to neurobiology and treatment in posttraumatic stress disorder. Depression and Anxiety, 16, 1438.

60 Etkin, A. \& Wager, T.D. (2007). Functional neuroimaging of anxiety: a meta-analysis of emotional processing in PTSD, social anxiety disorder, and specific phobia. American Journal of Psychiatry, 164, 14761488.

61 Kim, M.J. et al. (2011). The structural and functional connectivity of the amygdala:
From normal emotion to pathological anxiety. Behavioural Brain Research, 223, 403-410.

62 Hermans, E.J. et al. (2014). How the amygdala affects emotional memory by altering brain network properties. Neurobiology of Learning and Memory, 112, 2-16.

63 Richter-Levin, G. \& Maroun, M. (2010). Stress and Amygdala Suppression of Metaplasticity in the Medial Prefrontal Cortex. Cerebral Cortex, 20, 2433-2441.

64 Lanius, R.A., Frewen, P.A., Tursich, M Jetly, R. \& McKinnon, M.C. (2015). Restoring large-scale brain networks in PTSD and related disorders: a proposal for neuroscientifically informed treatment interventions. 2015, 6 .

65 Menon, V. (2011). Large-scale brain networks and psychopathology: a unifying triple network model. Trends in Cognitive Sciences, 15, 483-506.

66 Wager, T.D., Phan, K.L., Liberzon, I. \& Taylor, S.F. (2003). Valence, gender, and lateralization of functional brain anatomy in emotion: a meta-analysis of findings from neuroimaging. Neuroimage, 19 513-531.

67 Herrington, J.D. et al. (2005). Emotionmodulated performance and activity in left dorsolateral prefrontal cortex. Emotion, 5, 200-207.

68 Herrington, J.D. et al. (2010). Localization of asymmetric brain function in emotion and depression. Psychophysiology, 47, 442-454.

69 Engels, A.S. et al. (2010). Co-occurring anxiety influences patterns of brain activity in depression. Cognitive Affective \& Behavioural Neuroscience, 10, 141-156.

70 McMenamin, B.W. \& Marsolek, C.J. (2013). Can theories of visual representation help to explain asymmetries in amygdala function? Cognitive, Affective \& Behavioural Neuroscience, 13, 211-224.
71 Miller, G.A., Crocker, L.D., Spielberg, J. M., Infantolino, Z.P. \& Heller, W. (2013). Issues in localization of brain function: The case of lateralized frontal cortex in cognition emotion, and psycho-pathology. Frontiers in Integrative Neuroscience, 7, 2.

72 Jelicic, M., Geraerts, E., Merckelbach, H. \& Guerrieri, R. (2004). Acute stress enhances memory for emotional words, but impairs memory for neutral words. International Journal of Neuroscience, 114 1343-1351.

73 Smeets, T., Jelicic, M. \& Merckelbach, H. (2006). The effect of acute stress on memory depends on word valence. International Journal of Psychophysiology 62, 30-37.

74 Payne, J.D. et al. (2007). Stress administered prior to encoding impairs neutral but enhances emotional longterm episodic memories. Learning and Memory, 14, 861-868.

75 Sabatinelli, D., Lang, P.J., Bradley, M. M. Costa, V.D. \& Keil, A. (2009). The Timing of Emotional Discrimination in Human Amygdala and Ventral Visual Cortex. Journal of Neuroscience, 29, 14864-14868.

76 Pessoa, L. \& Adolphs, R. (2010). Emotion processing and the amygdala: from a 'low road' to 'many roads' of evaluating biological significance. Nature Reviews Neuroscience, 11, 773-782.

77 Joels, M., Pu, Z.W., Wiegert, O., Oitzl, M.S. \& Krugers, H.J. (2006). Learning under stress: How does it work? Trends in Cognitive Sciences, 10, 152-158.

78 Schwabe, L., Joels, M., Roozendaal, B. Wolf, O.T. \& Oitzl, M.S. (2011). Stress effects on memory: An update and integration. Neuroscience and Biobehavioural Reviews.

79 Cahill, L., Gorski, L. \& Le, K. (2003). Enhanced human memory consolidation with post-learning stress: interaction with the degree of arousal at encoding Learning and Memory, 10, 270-274.
80 Smeets, T., Otgaar, H., Candel, I. \& Wolf, O.T. (2008). True or false? Memory is differentially affected by stress-induced cortisol elevations and sympathetic activity at consolidation and retrieval. Psychoneuroendocrinology, 33, 13781386.

81 de Quervain, D.J., Roozendaal, B., Nitsch, R. M., McGaugh, J.L. \& Hock, C. (2000). Acute cortisone administration impairs retrieval of long-term declarative memory in humans. Nature Neuroscience, 3, 313-314.

82 Kuhlmann, S., Piel, M. \& Wolf, O.T. (2005). Impaired memory retrieval afte psychosocial stress in healthy young men. The Journal of neuroscience, 25 , 2977-2982.

83 Smeets, T. (2011). Acute stress impairs memory retrieval independent of time of day. Psychoneuroendocrinology, 36, 495501.

84 Schwabe, L., Joels, M., Roozendaal, B. Wolf, O.T. \& Oitzl, M.S. (2012). Stress effects on memory: an update and integration. Neuroscience and Biobehavioural Reviews, 36, 1740-1749.

85 Joels, M., Krugers, H. \& Karst, H. (2008), Stress-induced changes in hippocampal function. Progress in Brain Research, 167, 3-15.

86 Zoladz, P.R. et al. (2011). Pre-learning stress differentially affects long-term memory for emotional words, depending on temporal proximity to the learning experience. Physiology and Behaviour, 103, 467-476.

87 Schwabe, L. \& Wolf, O. (2014). Timing matters: Temporal dynamics of stress effects on memory retrieval. Cognitive Affective \& Behavioural Neuroscience, 14, 1041-1048.

88 Lang, P.J., Bradley, M.M. \& Cuthbert, B.N. (2008) International Affective Picture System (IAPS): Affective Ratings of 
Pictures and Instruction Manual, Technical Report A-8.

89 Cuthbert, B.N., Schupp, H.T., Bradley, M.M., Birbaumer, N. \& Lang, P.J. (2000). Brain potentials in affective picture processing: covariation with autonomic arousal and affective report. Biological Psychology, 52, 95-111.

90 Liu, Y.L., Huang, H.Q., McGinnis-Deweese, M., Keil, A. \& Ding, M.Z. (2012). Neural Substrate of the Late Positive Potential in Emotional Processing. Journal of Neuroscience, 32, 14563-14572.

91 Strelzyk, F. et al. (2012). Tune it down to live it up? Rapid, nongenomic effects of cortisol on the human brain. Journal of Neuroscience, 32, 616-625.

92 Schwabe, L., Wolf, O.T. \& Oitzl, M.S. (2010). Memory formation under stress: quantity and quality. Neuroscience and Biobehavioural Reviews, 34, 584-591.

93 Oitzl, M.S. \& Dekloet, E.R. (1992). Selective Corticosteroid Antagonists Modulate Specific Aspects of Spatial Orientation Learning. Behavioural Neuroscience, 106, 62-71.

94 Joels, M. \& Baram, T.Z. (2009). The neurosymphony of stress. Nature Reviews Neuroscience, 10, 459-U484.

95 Schwabe, L., Tegenthoff, M., Hoffken, O. \& Wolf, O.T. (2010). Concurrent glucocorticoid and noradrenergic activity shifts instrumental behaviour from goal-directed to habitual control. Journal of Neuroscience, 30, 8190-8196.

96 Davidson, R.J. (2004). What does the prefrontal cortex "do" in affect: perspectives on frontal EEG asymmetry research. Biological Psychology, 67, 219233.

97 Thibodeau, R., Jorgensen, R.S. \& Kim, S. (2006). Depression, anxiety, and resting frontal EEG asymmetry: a meta-analytic review. Journal of Abnormal Psychology, $115,715-729$.
98 Moscovitch, D.A. et al. (2011). Frontal EEG asymmetry and symptom response to cognitive behavioural therapy in patients with social anxiety disorder. Biological Psychology, 87, 379-385.

99 Davidson, R.J. (2000). Affective style, psychopathology, and resilience: Brain mechanisms and plasticity. American Psychologist, 55, 1196-1214.

100 Herwig, U., Satrapi, P. \& SchonfeldtLecuona, C. (2003). Using the international 10-20 EEG system for positioning of transcranial magnetic stimulation. Brain Topography, 16, 95-99.

101 Pizzagalli, D.A., Sherwood, R.J Henriques, J.B. \& Davidson, R.J. (2005). Frontal brain asymmetry and reward responsiveness - A source-localization study. Psychological Science, 16, 805-813.

102 Shackman, A.J., McMenamin, B.W., Maxwell, J.S., Greischar, L.L. \& Davidson, R.J. (2009). Right dorsolateral prefrontal cortical activity and behavioural inhibition. Psychological Science, 20, 1500-1506.

103 Gasser, T., Bacher, P. \& Steinberg, H. (1985). Test-retest reliability of spectral parameters of the EEG. Electroencephalography and Clinical Neurophysiology, 60, 312-319.

104 Tomarken, A.J., Davidson, R.J., Wheeler R.E. \& Kinney, L. (1992). Psychometric Properties of Resting Anterior EEG Asymmetry - Temporal Stability and Internal Consistency. Psychophysiology, 29, 576-592.

105 Klimesch, W., Schimke, H. \& Pfurtscheller, G. (1993). Alpha frequency, cognitive oad and memory performance. Brain Topography, 5, 241-251.

06 Doppelmayr, M., Klimesch, W., Pachinger, T. \& Ripper, B. (1998). Individual differences in brain dynamics: importan implications for the calculation of eventrelated band power. Biological Cybernetics, 79, 49-57.
107 Bazanova, O.M. \& Vernon, D. (2014). Interpreting EEG alpha activity. Neuroscience and Biobehavioural Reviews, 44C, 94-110.

108 Klimesch, W. (1999). EEG alpha and theta oscillations reflect cognitive and memory performance: a review and analysis. Brain Research Reviews, 29, 169-195.

109 Lewis, R.S., Weekes, N.Y. \& Wang, T.H. (2007). The effect of a naturalistic stressor on frontal EEG asymmetry, stress, and health. Biological Psychology, 75, 239-247.

110 Tops, M. et al. (2005). Acute cortiso administration modulates EEG alpha asymmetry in volunteers: relevance to depression. Biological Psychology, 69, 181-193.

111 Coan, J.A., Allen, J.J.B. \& McKnight, P.E. (2006). A capability model of individual differences in frontal EEG asymmetry. Biological Psychology, 72, 198-207.

112 Goodman, R.N., Rietschel, J.C., Lo, L.C., Costanzo, M.E. \& Hatfield, B.D. (2013) Stress, emotion regulation and cognitive performance: The predictive contributions of trait and state relative frontal EEG alpha asymmetry International Journal of Psychophysiology 87, 115-123.

113 Stewart, J.L., Coan, J.A., Towers, D.N. \& Allen, J.J.B. (2014). Resting and task elicited prefrontal EEG alpha asymmetry in depression: Support for the capability model. Psychophysiology, 51, 446-455.

114 Meyer, T. et al. (2014). Frontal EEG asymmetry as predictor of physiological responses to aversive memories. Psychophysiology.

115 Davidson, R.J. (1998). Affective style and affective disorders: Perspectives from affective neuroscience. Cognition \& Emotion, 12, 307-330.

116 Curtis, W.J. \& Cicchetti, D. (2007). Emotion and resilience: A multilevel investigation of hemispheric electro-encephalogram asymmetry and emotion regulation in maltreated and non-maltreated children Development and Psychopathology, 19, 811-840.

117 Putman, P. \& Roelofs, K. (2011). Effects of single cortisol administrations on human affect reviewed: Coping with stress through adaptive regulation of automatic cognitive processing Psychoneuroendocrinology, 36, 439-448.

118 Schutter, D.J.L.G. \& Harmon-Jones, E. (2013). The corpus callosum: A commissural road to anger and aggression. Neuroscience Biobehavioural Reviews, 37, 2481-2488.

119 Allen, J.J. \& Cohen, M.X. (2010). Deconstructing the "resting" state: exploring the temporal dynamics of frontal alpha asymmetry as an endophenotype for depression. Frontiers in Human Neuroscience, 4, 232.

120 Brookes, M.J. et al. (2011). Investigating the electrophysiological basis of resting state networks using magnetoencephalography. Proceedings of the National Academy of Sciences of the United States of America, 108, 16783-16788.

121 Wang, L., Saalmann, Y.B., Pinsk, M.A Arcaro, M.J. \& Kastner, S. (2012). Electrophysiological Low-Frequency Coherence and Cross-Frequency Coupling Contribute to BOLD Connectivity. Neuron, 76, 1010-1020.

122 Hanslmayr, S., Sauseng, P., Doppelmayr, M., Schabus, M. \& Klimesch, W. (2005). Increasing individual upper alpha powe by neurofeedback improves cognitive performance in human subjects. Applied Psychophysiology and Biofeedback, 30, 1

123 Klimesch, W., Sauseng, P. \& Gerloff, C. (2003). Enhancing cognitive performance with repetitive transcranial magnetic stimulation at human individual alpha frequency. European Journal of Neuroscience, 17, 1129-1133. 
124 Harmon-Jones, E. \& Allen, J.J.B. (1997). Behavioural activation sensitivity and resting frontal EEG asymmetry: Covariation of putative indicators related to risk for mood disorders. Journal of Abnormal Psychology, 106, 159-163.

125 Sutton, S.K. \& Davidson, R.J. (1997). Prefrontal brain asymmetry: A biological substrate of the behavioural approach and inhibition systems. Psychological Science, 8, 204-210.

126 Heffelfinger, A.K. \& Newcomer, J.W. (2001). Glucocorticoid effects on memory function over the human life span. Development and Psychopathology, 13, 491-513.

127 Kukolja, J., Thiel, C., Wolf, O. \& Fink, G (2008). Increased cortisol levels in cognitively challenging situations are beneficial in young but not older subjects. Psychopharmacology, 201, 293304.

128 Andreano, J.M. \& Cahill, L. (2009). Sex influences on the neurobiology of learning and memory. Learning and Memory, 16, 248-266.

129 Wolf, O.T., Schommer, N.C., Hellhammer D.H., McEwen, B.S. \& Kirschbaum, C. (2001). The relationship between stress induced cortisol levels and memory differs between men and women. Psychoneuroendocrinology, 26, 711-720.

130 Kirschbaum, C., Wust, S. \& Hellhammer, D. (1992). Consistent sex-differences in cortisol responses to psychological stress. Psychosomatic Medicine, 54, 648657.

131 Wang, J.J. et al. (2007). Gender difference in neural response to psychological stress. Social Cognitive and Affective Neuroscience, 2, 227-239.

132 Kirschbaum, C., Kudielka, B.M., Gaab, J. Schommer, N.C. \& Hellhammer, D. H. (1999). Impact of gender, menstrual cycle phase, and oral contraceptives on the activity of the hypothalamus-pituitary- adrenal axis. Psychosomatic Medicine, 61, 154-162.

133 Ossewaarde, L. et al. (2010). Neural mechanisms underlying changes in stress-sensitivity across the menstrua cycle. Psychoneuroendocrinology, 35, 4755.

134 Goldstein, J.M., Jerram, M., Abbs, B., Whitfield-Gabrieli, S. \& Makris, N. (2010). Sex differences in stress response circuitry activation dependent on female hormonal cycle. Journal of Neuroscience, 30, 431-438.

135 Kilpatrick, L.A., Zald, D.H., Pardo, J.V. \& Cahill, L.F. (2006). Sex-related differences in amygdala functional connectivity during resting conditions. Neuroimage, 30, 452-461.

136 Roelofs, K. et al. (2009). HypothalamusPituitary-Adrenal axis hyperresponsiveness is associated with increased social avoidance behaviour in social phobia. Biological Psychiatry, 65, 336-343.

137 Simeon, D. et al. (2007). Hypothalamicpituitary-adrenal axis function in dissociative disorders, post-traumatic stress disorder, and healthy volunteers. Biological Psychiatry, 61, 966-973.

138 MacMillan, H.L. et al. (2009). Cortisol Response to Stress in Female Youths Exposed to Childhood Maltreatment Results of the Youth Mood Project. Biological Psychiatry, 66, 62-68.

139 Pierrehumbert, B. et al. (2009). The influence of attachment on perceived stress and cortisol response to acute stress in women sexually abused in childhood or adolescence. Psychoneuroendocrinology, 34, 924-938.

140 Elzinga, B.M., Schmahl, C.G., Vermetten, E., van Dyck, R. \& Bremner, J.D. (2003). Higher cortisol levels following exposure to traumatic reminders in abuse-related PTSD. Neuropsychopharmacology, 28, 1656-1665.
141 Bremner, J.D. et al. (2003). Cortisol response to a cognitive stress challenge in posttraumatic stress disorder (PTSD) related to childhood abuse. Psychoneuroendocrinology, 28, 733-750.

142 Stoppelbein, L., Greening, L. \& Fite, P. (2012). The role of cortisol in PTSD among women exposed to a trauma-related stressor. Journal of Anxiety Disorders, 26, 352-358.

143 de Kloet, C.S. et al. (2006). Assessment of HPA-axis function in posttraumatic stress disorder: pharmacological and nonpharmacological challenge tests, a review. Journal of Psychiatric Research, 40 , 550-567.

144 Vyas, A., Mitra, R., Rao, B.S.S. \& Chattarji, S. (2002). Chronic stress induces contrasting patterns of dendritic remodeling in hippocampal and amygdaloid neurons. Journal of Neuroscience, 22, 6810-6818.

145 Liston, C. et al. (2006). Stress-induced alterations in prefrontal cortical dendritic morphology predict selective impairments in perceptual attentiona set-shifting. Journal of Neuroscience, 26 , 7870-7874.

146 Lee, T., Jarome, T., Li, S.J., Kim, JJJ \& Helmstetter, F.J. (2009). Chronic stress selectively reduces hippocampal volume in rats: a longitudinal magnetic resonance imaging study. Neuroreport, 20, 1554-1558.

147 Smeets, T. et al. (2012). Introducing the Maastricht Acute Stress Test (MAST): A quick and non-invasive approach to elicit robust autonomic and glucocorticoid stress responses. Psychoneuroendocrinology, 37, 1998-2008.

148 Zoefel, B., Huster, R. J. \& Herrmann, C.S. (2011). Neurofeedback training of the upper alpha frequency band in EEG improves cognitive performance. Neuroimage, 54, 1427-1431.
149 Dekker, M.K., Sitskoorn, M.M., Denissen A.J. \& van Boxtel, G.J. (2014). The timecourse of alpha neurofeedback training effects in healthy participants. Biological Psychology, 95, 70-73.

150 Huster, R.J., Mokom, Z.N., EnriquezGeppert, S. \& Herrmann, C.S. (2014). Brain-computer interfaces for EEG neurofeedback: peculiarities and solutions. International Journal of Psychophysiology, 91, 36-45.

151 Sherlin, L. et al. (2011). Neurofeedback and Basic learning Theory: Implications for Research and Practice. Journal of Neurotherapy: Investigations in Neuromodulation, Neurofedback and Applied Neuroscience, 15, 292-304.

152 Hagemann, D. (2004). Individual differences in anterior EEG asymmetry: methodological problems and solutions. Biological Psychology, 67, 157-182.

153 Baur, R., Conzelmann, A., Wieser, M.J. \& Pauli, P. (2015). Spontaneous emotion regulation: Differential effects on evoked brain potentials and facial muscle activity. International Journal of Psychophysiology, 96, 38-48.

154 MacNamara, A., Fod, D. \& Hajcak, G. (2009). Tell Me About It: Neural Activity Elicited by Emotional Pictures and Preceding Descriptions. Emotion, 9, 531 543.

155 Moser, J.S., Hajcak, G., Bukay, E. \& Simons, R.F. (2006). Intentional modulation of emotional responding to unpleasan pictures: An ERP study. Psychophysiology, 43, 292-296.

156 Bamford, S. et al. (2015). The late positive potential: a neural marker of the regulation of emotion-based approachavoidance actions? Biological Psychology, 105, 115-123.

157 Goebel, R. \& Linden, D. (2014) Neurofeedback with Real-Time Functional MRI. In Christoph Mulert \& 
Martha E. Shenton (Eds.), MRl in Psychiatry (pp. 35-46): Springer.

158 Zotev, V. et al. (2011). Self-Regulation of Amygdala Activation Using Real-Time fMRI Neurofeedback. PloS One, 6.

159 Johnston, S.J., Boehm, S.G., Healy, D. Goebel, R. \& Linden, D.E.J. (2010). Neurofeedback: A promising tool for the self-regulation of emotion networks. Neuroimage, 49, 1066-1072.

160 Sarkheil, P. et al. (2015). fMRI feedback enhances emotion regulation as evidenced by a reduced amygdala response. Behavioural Brain Research, 281, 326-332.

161 Bruhl, A. et al. (2014). Real-time Neurofeedback Using Functional MR Could Improve Down-Regulation of Amygdala Activity During Emotional Stimulation: A Proof-of-Concept Study. Brain Topography, 27, 138-148.

162 Halder, S. et al. (2011). Neura mechanisms of brain-computer interface control. Neuroimage, 55, 1779-1790.

163 Dasilva, F.L. (1991). Neural Mechanisms Underlying Brain Waves - from Neural Membranes to Networks. Electroencephalography and Clinical Neurophysiology, 79, 81-93.

164 Harmon-Jones, E., Harmon-Jones, C. Fearn, M., Sigelman, J.D. \& Johnson,P. (2008). Left frontal cortical activation and spreading of alternatives: Tests of the action-based model of dissonance. Journal of Personality and Social Psychology, 94, 1-15.

165 Ros, T. et al. (2013). Mind over chatter: plastic up-regulation of the fMRI salience network directly after EEG neurofeedback. Neuroimage, 65, 324-335.

66 Hermans, E.J. et al. (2011). Stress-related noradrenergic activity prompts largescale neural network reconfiguration. Science, 334, 1151-1153.

67 Henckens, M.J.A.G., van Wingen, G.A Joels, M. \& Fernandez, G. (2012). Time- dependent effects of cortisol on selective attention and emotional interference: a functional MRI study. Frontiers in Integrative Neuroscience, 6, 66.

168 Henckens, M.J.A.G., van Wingen, G.A. Joels, M. \& Fernandez, G. (2010). Timedependent effects of corticosteroids on human amygdala processing. Journal of Neuroscience, 30, 12725-12732.

169 Birn, R.M., Murphy, K., Handwerker, D.A. \& Bandettini, P.A. (2009). fMRI in the presence of task-correlated breathing variations. Neuroimage, 47, 1092-1104.

170 Shmueli, K. et al. (2007). Low-frequency fluctuations in the cardiac rate as a source of variance in the resting-state $\mathrm{FMRI} B O L D$ signal. Neuroimage, 38, 306-320.

171 Wang, J.et al. (2005). Perfusion functiona MRI reveals cerebral blood flow pattern under psychological stress. Proceedings of the National Academy of Sciences of the United States of America, 102, 1780417809.

172 Canli, T. et al. (2001). An fMRI study of personality influences on brain reactivity to emotional stimuli. Behavioural Neuroscience, 115, 33-42.

173 Hauer, D. et al. (2011). Relationship of a common polymorphism of the glucocorticoid receptor gene to traumatic memories and posttraumatic stress disorder in patients after intensive care therapy. Critical Care Medicine, 39, 643-650.

174 Ackermann, S., Heck, A., Rasch, B. Papassotiropoulos, A. \& de Quervain, D.J. (2013). The Bcll polymorphism of the glucocorticoid receptor gene is associated with emotional memory performance in healthy individuals. sychoneuroendocrinology, 38, 12031207.

175 Wust, S. et al. (2004). Common polymorphisms in the glucocorticoid receptor gene are associated with adrenocortical responses to psychosocial stress. Journal of Clinical Endocrinology and Metabolism, 89, 565-573.

176 DeRijk, R.H. et al. (2006). A common polymorphism in the mineralocorticoid receptor modulates stress responsiveness. Journal of Clinical Endocrinology and Metabolism, 91, 50835089.

177 Bachmann, A.W. et al. (2005) Glucocorticoid receptor polymorphisms and post-traumatic stress disorder. Psychoneuroendocrinology, 30, 297-306.

178 van Rossum, E.F. et al. (2006) Polymorphisms of the glucocorticoid receptor gene and major depression Biological Psychiatry, 59, 681-688.

179 Struber, N., Struber, D. \& Roth, G. (2014). Impact of early adversity on glucocorticoid regulation and later mental disorders. Neuroscience and Biobehavioural Reviews, 38, 17-37.

180 Elzinga, B.M. et al. (2008). Diminished cortisol responses to psychosocial stress associated with lifetime adverse events A study among healthy young subjects. Psychoneuroendocrinology, 33, 227-237.

181 Lovallo, W.R., Farag, N.H., Sorocco, K. H. Cohoon, A.J. \& Vincent, A.S. (2012). ifetime Adversity Leads to Blunted Stress Axis Reactivity: Studies from the Oklahoma Family Health Patterns Project. Biological Psychiatry, 71, 344-349.

182 Cryan, J.F. \& Dinan, T.G. (2013). Unraveling the Longstanding Scars of Early Neurodevelopmental Stress. Biological Psychiatry, 74, 788-789. 


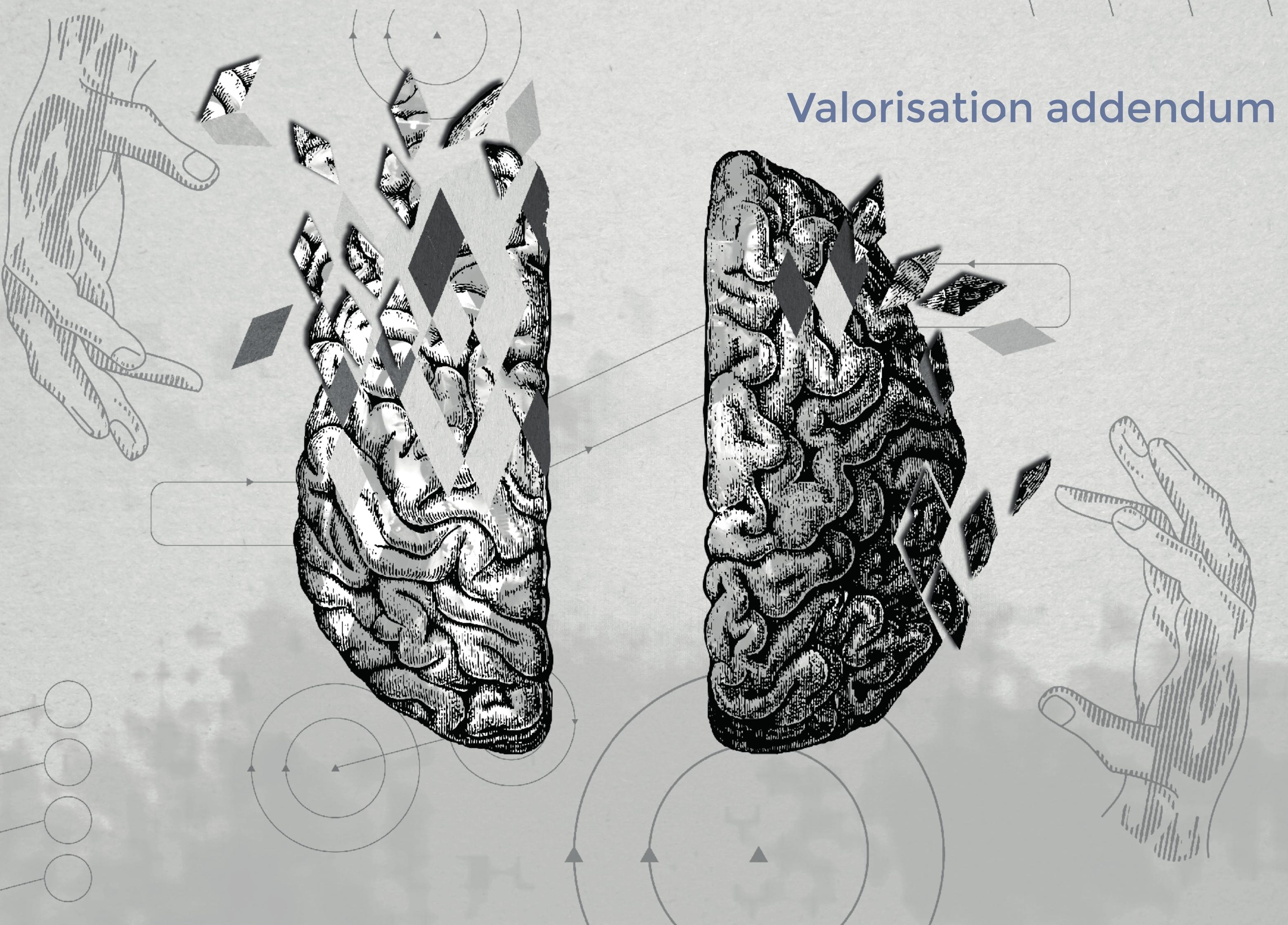


The work presented in this dissertation aimed to identify the changes in neural responding that underlie adaptability after exposure to acute stress, i.e., changes that are said to characterize stress resilience. The theoretical and practical implications of our findings were described in the various chapters throughout this thesis. Below, our findings are discussed in a broader societal context.

\section{Societal relevance}

We all know how it feels to be stressed. Our lives are filled with daily hassles such as rushing for an appointment for which you are already late, managing deadlines at work, taking an exam at school or giving a presentation in front of a large audience. Even planning a holiday or going to the dentist can be daunting to some of us. Besides, about half of all adults experiences at least one severe stressor in their life (e.g., violence, abuse, a life threatening accident). In all forms, prolonged exposure to stress has adverse effects on mental functions and makes us vulnerable for mental disorders. Mood and anxiety disorders are examples of a loss of resilience and are accompanied by social disabilities and high societal costs. Depression and post-traumatic stress disorder (PTSD) are characterized by changes in brain circuitry and function. These neural alternations probably result from both stress vulnerability and acute stressors that precipitate the disorder. Still, the exact changes in brain function leading to stress-related psychopathology are hitherto not well understood, hampering the development of novel therapeutic interventions. Moreover, the current psychological and pharmacological treatments are efficacious in only approximately half of the patients, thus leaving much room for improvement.

Learning why some individuals are better able to cope with stressful events than others is important to inform intervention and prevention programs targeting stress resilience. We therefore explored the possibility to modify cortical brain activity via neurofeedback to open a window of opportunity that may boost the efficacy of the other treatments. The development of prevention programs is particularly significant for professionals who have a heightened risk of exposure to stressful life events, such as emergency workers, police officers, and military personnel. Overall, the main aim of this thesis was to identify changes in the brain associated with acute stress. The next step is to understand how exactly these brain processes impact psychological functions and increase 
the risk for mental disorders. This requires collaborative efforts from experimental psychology, neuroimaging and psychiatry.

\section{Scientific perspectives and innovation}

Abnormalities in the regulation of the neuroendocrine stress system have been linked to an increased susceptibility to psychopathology and disease after a stressful experience. There is substantial evidence of an altered functioning of the neuroendocrine system in depression (i.e., distorted basal cortisol levels) and PTSD (i.e., lowered basal cortisol levels). Therefore, empirical knowledge on how stress and stress hormones affect brain function can inform us about the aetiology and mechanisms in stress-related psychopathology. A first step in achieving this is reaching a better understanding of the dynamic acute stressinduced changes in processing in the brain that underlie adaptability after exposure to acute stress.

We investigated the reactivity of the stress system in a multidisciplinary way by addressing the interplay between psychophysiological markers (e.g., cortisol) and brain substrates underlying stress resilience. Using the definition of resilience as the ability to recover swiftly after a stressful life event, we investigated the contribution of different brain areas in post-stress regulation. To this end, we adapted a procedure used in experimental psychology during neuroimaging (i.e., the iMAST) that allows investigating the contribution of different brain areas during different phases in stress regulation. In particular, by looking at changes in amygdala connectivity, this work reveals a dynamic brain mechanism that is regulated by cortisol and involved in coping with acute stress. A particularly innovative aspect of this study is that it included repeated resting-state assessments enabling the investigation of post-stress brain activation changes during different phases after acute stress exposure.

Based on this innovative design, we were able to show that in the immediate stress phase, cortisol moderated the connectivity in the core areas of the salience network. Particularly, we found an enhanced amygdala - vmPFC connectivity in cortisol responders during immediate stress. This, together with the enhanced amygdala - vmPFC connectivity found in disorders marked by HPA-axis dysregulation like PTSD, suggests that the connectivity between the amygdala and vmPFC might characterize vulnerability during $a$ state of acute stress. During the early recovery, a cortisol moderated time-dependent shift in the dorsal control network was found. Cortisol responders were characterized by reduced amygdala-left dIPFC connectivity during stress recovery. Combined with the findings that depressed patients show an impaired cortisol recovery pattern and display right-sided lateralized frontal brain activity, this points toward a cortisol mediated left dIPFC regulatory circuit involved in adaptive recovery from stress. An important objective for future research will be the use of longitudinal designs, to determine whether acute stress related functional connectivity is useful in predicting clinical outcomes.

Another hallmark feature of PTSD, besides dysregulation of the stress system are disturbances of memory. Therefore, basic knowledge about how stress hormones affect memory processes, might have implications for the understanding and treatment of anxiety disorders. We investigated the time-dependent cortisol effects on memory encoding and found that timing of stress exposure influenced the direction of the association between the cortisol response and the total number of remembered pictures 24 hours later. The number of correctly recognized neutral pictures was positively associated with cortisol responses in the immediate stress condition, whereas it was negatively associated within the 30-min prelearning stress condition. Our results indicate that it is crucial to carefully take the time lag into account when considering cortisol as additional treatment during exposure techniques that are part of cognitive-behavioural therapies for PTSD.

In recent years, important insights into the putative functional role of oscillatory brain activity for cognitive processes and emotional states have been gained. For instance, it has been shown that frontal hemispheric asymmetry in the alpha band is linked to the processing of emotions. We demonstrated for the first time that brain asymmetry of the frontal cortex plays a functional role in acute stress processing (i.e., relative left frontal baseline activity resulted in a smaller task-induced cortisol response). This corresponds wel with prior research showing that alternations in frontal asymmetry are associated with features of stress-related disorders, such as depression. Together, these findings suggest that frontal asymmetry is a potential target for interventions aimed at increasing resilience.

EEG neurofeedback is a method that could be used to modulate frontal asymmetry. EEG neurofeedback is based on real-time analysis of EEG signals. The use of real-time analysis of ongoing brain activity enables us to provide individuals with feedback information about their brain activity, which can then be used to directly train specific parameters of brain 
activity and consequently change behavior. We aimed to validate and explore the potential of frontal alpha asymmetry neurofeedback and its behavioural usefulness. A particula strength of our study is that we compared two frontal asymmetry protocols with a placebo condition. Moreover, we extended prior studies by determining frontal asymmetry for each participant based on individual alpha peak frequency and by including two follow-up measurements (i.e., one week and one month later). Individual frontal alpha frequency neurofeedback resulted only in a change in relative frontal asymmetry at rest in participants who were trained to increase relative right-sided frontal alpha asymmetry. This change in relative frontal alpha asymmetry seemingly intensified subjective stress experiences. Importantly, our results indicate that there are marked individual differences in the ability to learn how to self-regulate frontal asymmetry. Ensuring reproducibility both on the individual level and over time is a key challenge in neurofeedback studies. Investigating the effectiveness of EEG neurofeedback using a placebo controlled designed and by comparing it with other therapies (i.e., psychotherapy, pharmacological treatment) is important before any translation to a more applied context is justified.

\section{Translation and knowledge dissemination}

This dissertation describes studies looking into the neural correlates of acute stress processing in the brain and may thus be of interest to members of the scientific community working in various fields, including affective neuroscience, emotion and memory. Given the stage of imaging in stress research and the fact that the current findings warrant replication, the main focus is on knowledge transfer within the scientific community. The findings of our studies have been communicated via publications in peer-reviewed journals. Moreover, the knowledge that was acquired while conducting the studies has been communicated via several presentations on international research conferences and meetings of the National Initiative Brain and Cognition (e.g., brain product day; explore your research day; meetings of the innovative programme resilience and vulnerability following stress). Additionally, several lectures (e.g., Maastricht University) and workshops were given about the application of neurofeedback (e.g., first meeting focus area Safety in Utrecht; 2nd Forensic Psychology Update in Maastricht). Furthermore, transfer of the practical skills that were acquired in the process of conducting the studies has taken place via teaching (i.e., practical; internships). Knowledge transfer outside the scientific community occurred via demonstrations on the university open day. Finally, communication of the findings of the studies in this thesis outside the scientific community will be done via a press release of NWO. 


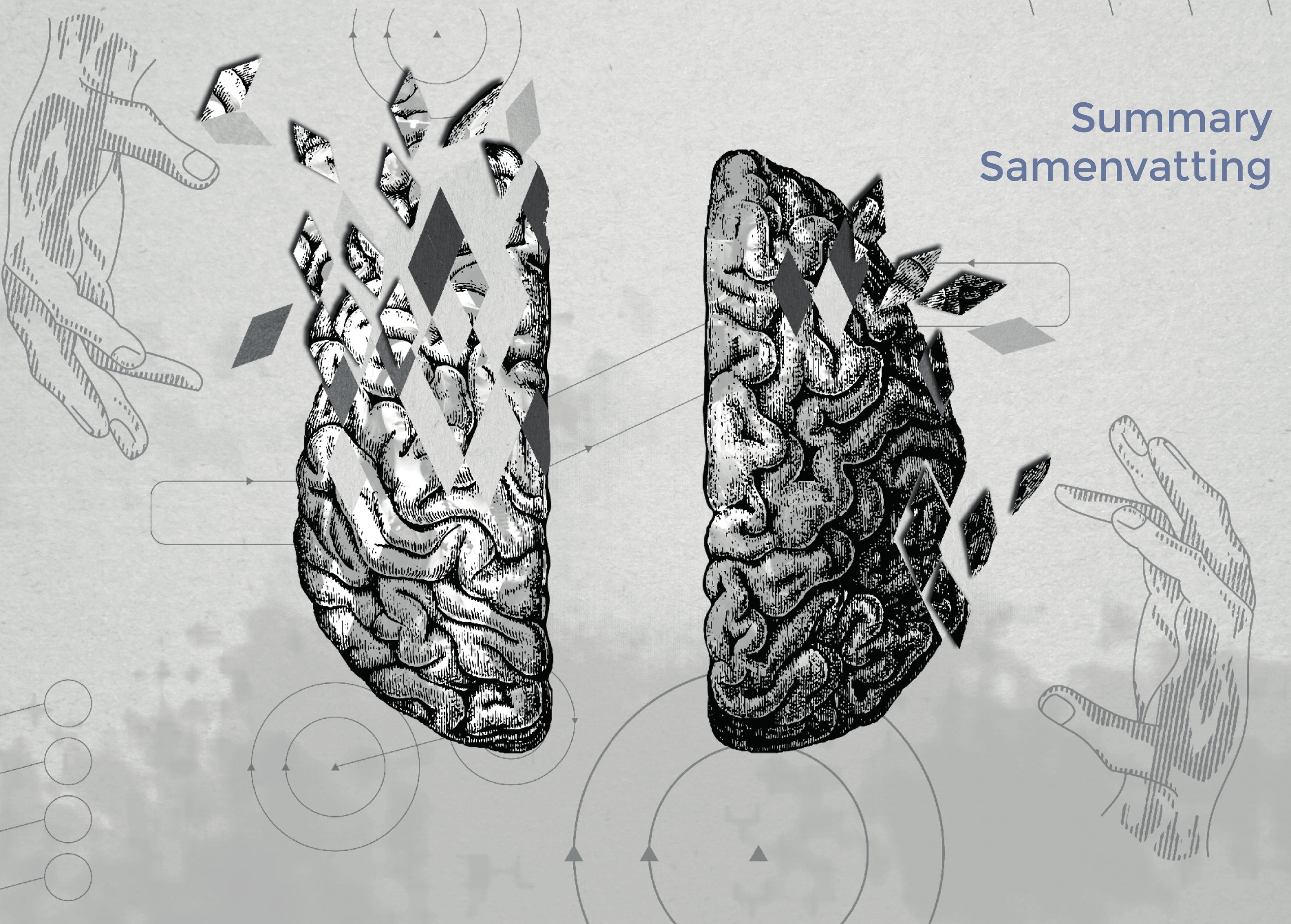




\section{Summary}

Stress resilience is the ability to recover swiftly after a stressful life event. It depends on the nature and intensity of the stressor interacting with the ability of the individual to cope with the experience. To help identify what makes people thrive in the face of adverse life events, it is imperative to investigate the contribution of different brain areas in post-stress regulation. In this thesis, changes in neural responding to acute stress processing were investigated with a focus on the physiological cortisol response. Hypothalamic-pituitaryadrenal (HPA) axis reactivity has been linked to a wide range of mental disorders. Therefore, basic knowledge on how stress and stress hormones affect brain function can inform us about the aetiology and mechanisms in stress-related psychopathology. A first step in achieving this, is reaching a better understanding of the dynamic acute stress-induced changes in processing in the brain that underlie adaptability after exposure to acute stress.

Using the definition of resilience as the ability to recover swiftly after a stressful life event, we investigated the contribution of different brain areas in post-stress regulation. One of the driving forces in this thesis was the hypothesis that stress hormones trigger a time-dependent shift in neuronal resource to promote behavioural adaptation in various phases after acute stress. To this end, we developed a stress task (i.e., the iMAST) that allows for investigating the contribution of different brain areas during different phases in stress regulation (i.e., stress reactivity and recovery). By looking at changes in amygdala connectivity, this work reveals a dynamic brain mechanism that is regulated by cortisol and involved in coping with acute stress. In the immediate stress phase, we observed a cortisol moderated time-dependent shift in the core areas of the salience network. Particularly, we found an enhanced amygdala - vmPFC connectivity in cortisol responders during immediate stress. This, together with the enhanced amygdala - vmPFC connectivity found in disorders marked by HPA-axis dysregulation like PTSD, suggests that the connectivity between the amygdala and vmPFC might characterize vulnerability during a state of acute stress. During the early recovery, a cortisol moderated time-dependent shift in the dorsal control network was found. Cortisol responders were characterized by reduced amygdala-left dIPFC connectivity during stress recovery. Combined with the findings that depressed patients show an impaired cortisol recovery pattern and show right-sided lateralized frontal brain activity, this points toward a cortisol mediated left dIPFC regulatory circuit involved in adaptive recovery from stress. 
The right lateralized characteristic brain activity profile in depression was the main impetus to assess the functional role of frontal alpha asymmetry in acute stress responding and to investigate the validity of individualized alpha EEG neurofeedback targeted at modulating the asymmetry of the frontal cortex to change stress responding. Relative left frontal baseline activity resulted in a smaller task-induced cortisol response. In addition, individual frontal alpha frequency neurofeedback resulted in a change in relative frontal asymmetry at rest in participants in the right group, and this change in relative frontal alpha asymmetry seemingly intensified subjective stress experiences. Importantly, our results indicate that there are marked individual differences in the ability to learn how to selfregulate frontal asymmetry. Ensuring reproducibility both on the individual level and over time is a key challenge in neurofeedback studies.

Eliciting stress prior to encoding seems a promising avenue to test and extend the temporal dynamics model of stress effects on memory formation. We found that timing of stress exposure influenced the direction of the association between the cortisol response and the amount of remembered pictures 24 hours later. Correctly recognized neutral pictures were positively associated with cortisol responses in the immediate stress condition, whereas they were negatively associated within the 30-min pre-learning stress condition. Stress-related disorders are characterized disturbances of memory and by alternations in the stress response. Our results indicate that in this context, the timedependent actions of cortisol are of relevance, pointing toward secondary prevention possibilities.

All in all, this thesis provides insights in the dynamic brain mechanisms that underlie stress resilience. Most importantly, we developed a task that enables researchers to investigate the role of cortisol in dynamically changing the contribution of different brain areas during various phases in acute stress regulation. Specifically, the amygdala - vmPFC and amygdala - dIPFC are modulated by acute stress in a time specific manner relevant for adaptive stress coping. Frontal asymmetry, possibly originating in the dIPFC, modulates the cognitive processing of threatening information as well as the fight-or-flight response in a state-dependent manner. Our research provokes new questions, and we hope that in the near future it will become apparent whether different resilience and vulnerability factors converge on common brain mechanisms.

\section{Samenvatting}

Vrijwel iedereen ervaart geregeld enige mate van stress. Onze hedendaagse maatschappij kent vele uiteenlopende gebeurtenissen die een stressreactie kunnen uitlokken, zoals dagdagelijkse taken en afspraken, het halen van bijna onmogelijke deadlines op het werk, of problemen in de relationele sfeer. De hoeveelheid stress die bij dit soort gebeurtenissen wordt ervaren hangt af van persoon tot persoon. Het vermogen goed om te kunnen gaan met, en snel te kunnen herstellen van, deze stressvolle gebeurtenissen noemen we veerkracht ("resilience"). Het doel van dit proefschrift is om de veranderingen in het brein die kenmerkend zijn voor stressbestendigheid, te identificeren en daarmee bij te dragen aan ons inzicht in stress-gerelateerde psychische aandoeningen. Ons brein reguleert de acute stressreactie, waarbij de situatie als bedreigend wordt herkend en waardoor (stress)hormonen vrijgegeven worden. Deze stressreactie zorgt er op zijn beurt voor dat we adequaat kunnen reageren tijdens een stressvolle situatie. Deze hormonen werken daarbij ook in op hersengebieden die een belangrijke rol spelen bij gedrag en emoties, zoals de amygdala, hippocampus en prefrontale cortex. Om de effecten van stresshormonen op het brein te onderzoeken hebben we beeldvormende technieken (EEG en functionele MRI) gecombineerd met het experimenteel onder stress brengen van onze proefpersonen. In deel 1 van dit proefschrift, getiteld "Imaging the stressed brain", hebben we de specifieke temporele effecten van acute stress op de communicatie tussen de amygdala en andere hersengebieden (functionele hersenconnectiviteit) bestudeerd. Daarnaast hebben we gekeken naar de tijdsafhankelijke effecten van stress op het geheugen waarbij we hersenactiviteit (EEG) gemeten hebben tijdens het leren van afbeeldingen.

Het efficiënt induceren van stress tijdens beeldvormend hersenonderzoek is een hele uitdaging. Gezien de prominente rol van acute stressinductie in dit proefschrift, was de eerste stap dan ook gericht op het valideren van een adequate methode om acute stress uit te lokken (Hoofdstuk 1). Wij deden dit aan de hand van de imaging Maastricht Acute Stress Test (iMAST), die bestaat uit een combinatie van fysieke en psychologische stress. De fysieke stress wordt ontlokt door kortstondige, maar evenwel sterke koude stimulaties op de hand van de proefpersonen. De psychologische stress bestaat eruit een moeilijke hoofdrekentaak te combineren met sociale evaluatie. De iMAST werd door het merendeel van onze proefpersonen als zeer stressvol ervaren en resulteerde in duidelijk verhoogde niveaus van 
stresshormonen (cortisol en het sterk met noradrenaline samenhangende enzym alphaamylase).

Tijdens de acute stressfase zijn noradrenaline en cortisol beide actief in het brein. Je wordt voorbereid op een vechten- of vluchtreactie. Eens de stressvolle gebeurtenis voorbij is speelt cortisol een rol bij het kiezen van passend gedrag en bij het normaliseren van het brein. In hoofdstuk 2 hebben we gekeken naar de effecten van acute stress op veranderingen van de functionele connectiviteit van de amygdala tijdens verschillende fases na stress. De amygdala is een van de eerste hersengebieden die actief wordt als we worden geconfronteerd met een lichamelijk en/of psychisch stressvolle gebeurtenis. We hebben bij gezonde deelnemers de connectiviteit van de amygdala drie keer gemeten: voor, onmiddellijk na (acute stressfase) en 30-min na (vroege herstelfase) acute stressinductie met de iMAST. De connectiviteit tussen de amygdala en de mediale prefrontale cortex (mPFC), gemeten voor de stressinductie in rust, voorspelde de latere taak gerelateerde cortisol toename. Een sterkere connectiviteit resulteerde in lagere cortisolwaardes. Daarnaast vonden we een verschil in amygdala connectiviteit tussen individuen die een lage of een hoge toename in cortisol vertoonden (cortisol responders). Tijdens de acute stressfase werden cortisol responders gekenmerkt door versterkte connectiviteit tussen de amygdala en de ventromediale prefrontale cortex (vmPFC). Deze versterkte amygdala-vmPFC connectiviteit is in voorgaande onderzoeken ook gevonden in stress-gerelateerde stoornissen zoals post-traumatische stressstoornis (PTSS), hetgeen erop wijst dat deze versterkte connectiviteit een mogelijke kwetsbaarheid zou kunnen zijn tijdens acute stress. Tijdens de vroege herstelfase na stress werden cortisol responders gekenmerkt door versterkte connectiviteit tussen de amygdala en de hippocampus, een verzwakte connectiviteit tussen de amygdala en de dorsale anterieure cingulate cortex (dACC) en de linker dorsolaterale prefrontale cortex (dIPFC). De verminderde connectiviteit met de dACC en linker dIPFC kan wijzen op minder effectieve manier van omgaan met (coping) en herstel van stress. Deze bevindingen doen ons denken aan de rechtszijdige laterale frontale activiteit en een verminderde afname van cortisol zoals die gezien wordt bij patiënten met een depressie. De verstrekte connectiviteit met de hippocampus na stress draagt mogelijk ook bij aan een beter geheugen voor stressvolle gebeurtenissen.

Gebaseerd op de theorie dat de exacte temporele relatie tussen het vrijkomen van de stresshormonen en leren cruciaal is voor het verbeterde geheugen voor stressvolle gebeurtenissen, hebben we in Hoofdstuk 3 gekeken naar de tijdsafhankelijke effecten van stress op het langetermijngeheugen. Hierbij hebben we de onderliggende mechanismen onderzocht door het meten van hersenactiviteit (EEG) tijdens het leren van afbeeldingen. De proefpersonen werden hetzij onmiddellijk, hetzij 30-min, voor het leren van neutrale en negatieve afbeeldingen onderworpen aan de Maastricht Acute Stress Test (MAST). Het langtermijngeheugen voor deze afbeeldingen werd 24-uur later getest. Wij vonden dat de prestatie op de geheugentest niet verschillende tussen de controle- en de stressconditie. Wel vonden we dat het verband tussen de hoeveelheid onthouden neutrale plaatjes en de cortisolresponse totaal verschillend was in de onmiddellijke versus de 30-min conditie. In overeenstemming met wat we theoretisch zouden verwachten, bleek dat in de onmiddellijke stressconditie een hoger cortisolniveau gerelateerd was aan het beter onthouden van de neutrale afbeeldingen. In de 30-min conditie was een hoger cortisolniveau echter gerelateerd aan het slechter onthouden van de neutrale afbeeldingen. Deze tijdspecifieke relatie met cortisol was bovendien gerelateerd aan de grootte van de amplitude van een elektrofysiologische index van aandacht en visuele verwerking die bekend staat als de Late Positive Potential (LPP). We vonden een negatief verband tussen de amplitude van de LPP en het niveau van cortisol in de 30-min stressconditie. De gecombineerde gedrags- en elektrofysiologische data suggereren dat bij hoge stressniveaus het leren in de 30-min conditie slechter was door een gecompromitteerd onderliggend aandachtsmechanisme.

In deel $\mathbf{2}$ van het proefschrift, getiteld "Brain asymmetry and stress resilience", hebben we gekeken naar de functionele rol van de frontale alfa asymmetrie in stressbestendigheid en hebben we de mogelijkheid onderzocht om frontale asymmetrische hersenactiviteit te veranderen via zelfregulatie (EEG neurofeedback) om zo stressbestendigheid te verhogen. In hoofdstuk 4 vonden we dat frontale alfa hersenasymmetrie gemeten met EEG een rol speelt in de stressreactie. Frontale alfa asymmetrie werd gemeten voor en meteen na acute stress die werd geïnduceerd door de MAST. Hierbij hebben we een vergelijking gemaakt tussen frontale alfa asymmetrie gebaseerd op de standaard $8-13 \mathrm{~Hz}$ band en een band gebaseerd op de individuele alfa piek. Relatieve linkszijdige frontale asymmetrie resulteerde in een lagere cortisolresponse, evenwel alleen indien de frontale asymmetrie gebaseerd was 
op de individuele alfa band. We vonden geen associatie tussen baseline frontale asymmetrie en subjectieve stress.

We waren ook geïnteresseerd in de mogelijkheid om frontale alfa asymmetrie te veranderen door middel van EEG neurofeedback (Hoofdstuk 5). Neurofeedback is gebaseerd op een online analyse van de huidige hersenactiviteit die omgezet wordt in visuele feedback. In een experimentele studie hebben we extensief drie neurofeedbackcondities vergeleken: trainen naar meer relatieve rechtszijdige frontale asymmetrie, trainen naar relatieve linkszijdige frontale asymmetrie, of een placebo controlegroep. Verder hebben we gekeken of de richting van verandering in frontale asymmetrie invloed had op de cortisol en subjectieve stress response zoals geïnduceerd met de MAST. Alleen proefpersonen in de rechtszijdige conditie waren in staat om hun frontale asymmetrie te veranderen, waarbij de verandering gepaard ging met verhoogde subjectieve stress. Belangrijk is dat onze resultaten grote individuele verschillen aantonen in het vermogen om je eigen frontale asymmetrie te leren veranderen. Daarnaast is reproduceerbaarheid over tijd een belangrijke uitdaging in neurofeedback studies.

Tot slot worden de belangrijkste resultaten en conclusies van de studies uit het proefschrift besproken in de algemene discussie. Veder worden parallellen tussen de resultaten van de verschillende hoofdstukken getrokken en suggesties voor verder onderzoek gedaan. Samenvattend kunnen we stellen dat cortisol een cruciale rol lijkt te spelen in de normalisatie van het brein na acute stress, en dat situatie-specifieke frontale asymmetrie belangrijk is bij de verwerking van dreigende informatie. Deze bevindingen, die uitgebreid beschreven staan in dit proefschrift, hebben daarbij geleid tot een beter inzicht in de dynamische hersenmechanismen die ten grondslag liggen aan stressbestendigheid. 


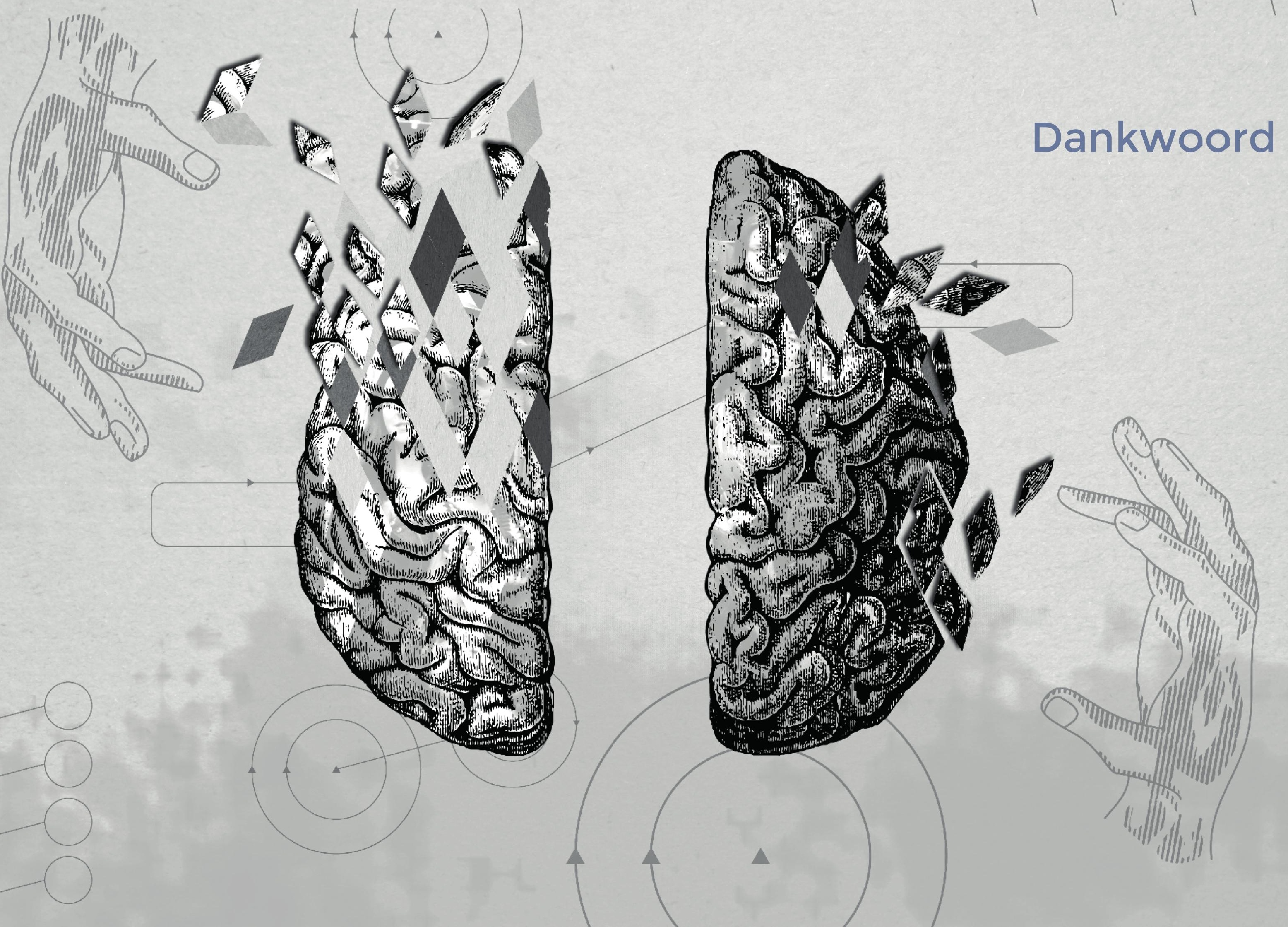


Mijn promotieonderzoek heeft mij de afgelopen 4,5 jaar veel leerzame en leuke ervaringen gebracht. Een dankjewel aan iedereen die aan deze onvergetelijke periode heeft bijgedragen. In het bijzonder:

Mijn begeleiders Harald en Tom. Allereest wil ik jullie bedanken voor deze kans die jullie me geboden hebben en de vrijheid die ik binnen het project kreeg. Harald, de nauwkeurigheid en snelheid waarmee jij leest is ronduit indrukwekkend. Daarnaast is je wetenschappelijk inzicht grenzeloos, ik hoop dat ik ooit ook zoveel literaire kennis mag vergaren als jij bezit. Tom, onze samenwerking heeft me enorm verrijkt. De kennis die ik door jouw begeleiding op het gebied van stressonderzoek heb kunnen opdoen is van onschatbare waarde. Ik wil je bedanken voor je vertrouwen, de aanmoedigingen om, naast de neurofeedback studie, aan wat kleinere projecten te werken en het stimuleren van mijn zelfstandigheid. Je was altijd beschikbaar voor vragen, al had dit tot gevolg dat ik in het begin iedere dag op je kantoor stond voor een sessie 'hardop nadenken'. Je beantwoordde mijn, vaak te lange, e-mails snel en gaf me altijd constructieve feedback op de artikelen. Ware luxe en ook zeer bepalend voor de hoeveelheid wetenschappelijke output (al is het misschien niet ten goede gekomen aan mijn ongeduldigheidstraining). Tom, ik kan je niet genoeg bedanken voor je harde werk, je toewijding en steun in de afgelopen jaren. Een betere begeleider had ik me niet kunnen wensen. Ik vind het fijn dat we onze samenwerking blijven voorzetten!

Thomas, ik heb genoten van ons teamwork. Ik vond je meteen al bizar slim en we hebben heel wat tijd discussiërend over frontale asymmetrie en andere interessante onderwerpen samen doorgebracht. Jij bent de perfecte combinatie van een wetenschapper en een filosoof. Ik waardeer je rust, geduld en grondigheid in alles wat je doet. Ik ben er zelfs een beetje jaloers op. Of het nu het lezen van mijn stukken was, het plakken van EEG of het delen van co-auteurschappen, je stond altijd voor mij klaar. Ook dank dat je me op mijn promotiedag wilt bijstaan. Blijf zo als je bent Thomas! Onze paden zullen zich vast nog vaak kruisen in de toekomst.

Graag wil ik ook een aantal collega's bedanken voor de fijne samenwerking. Inge, al sinds onze studietijd is duidelijk dat we onze passie voor onderzoek met elkaar delen. Het was leuk om samen te brainstormen, prestaties te vieren of gewoon te kletsen onder het genot 
van een kop koffie. Je directe bijdrage aan dit proefschrift waardeer ik zeer. Anke S, je was degene die me introduceerde in de wereld van de wetenschap. De kans die jij me hebt gegeven in Finland heeft aan de basis gestaan van waar ik nu sta. Fren, je elektrofysiologische kennis en nauwkeurigheid hebben een belangrijke bijdrage geleverd aan het neurofeedbackproject. Nicolette, je hebt veel inspanningen in het begin van het fMRI-project verricht. Samen met Vincent heb je veel vragen over de fMRI analyse beantwoordt. Frenk en Richel, jullie hebben ons mega neurofeedback project een vliegensvlugge start gegeven. Jacco, met je hulp bij het programmeren van de neurofeedbacksoftware en de werkgeheugentaak heb je een belangrijke bijdrage geleverd. Timo, jij liet me al snel kennis maken met het wereldje van publiceren. Dennis, bedankt voor de leuke studiejaren en de gezellige lunches. Sinikka and Eija, you made me feel so welcome in Finland, an international experience like this is a delight. Lars, thanks for providing feedback on the stress-timing paper and rubicon proposal and for giving me the opportunity to come to Hamburg.

Ik wil ook de collega's van Klinische Psychologie bedanken voor de gezelligheid. De Forensische groep, bedankt voor de gezellige koffie momenten, lunches, borrels en retraites. BuufMaartje, de auto- en (af en toe) fietsritjes naar de uni waren gezellig. Nathalie, mijn pingpong maatje, Elly en Colinde, ik kijk uit naar een spelletjesavond in Hamburg. Mijn "EPProomies" Lotte en Suzanne, de congressen waren gezellig. Mijn kamergenootjes Marjolein, Yvo, Henry, Elke, Jessica, Ghislaine, Karolien, Peggy, Irena, en mijn fMRI buddie Linda, dankzij jullie was het een genot om iedere dag naar kantoor te komen.

Graag dank ik ook mijn stagiaires Jonny, Michelle, Sofie, Celine, Phyllis en in het bijzonder Loran en Marijke, voor jullie inzet, toewijding en hulp bij het verzamelen van de data. Phyllis, bedankt voor de lekkere koekjes tijdens de vele gezellige scanuurtjes en je hulp bij mijn start in Hamburg. Bedankt aan de leden van de leescommissie en coronaleden voor hun tijd en bijdrage aan deze promotie. Johan, bedankt voor de bijzonder mooie artistieke cover

Tot slot dank aan (schoon)familie en vrienden, voor jullie steun. Cor en Elly, bedankt dat jullie altijd voor ons klaar staan. Cor, wat zouden we moeten zonder je talent en passie voor klussen. Elly, de verjaardagsuitjes houden we erin. Jacky, het is altijd fijn om een dokter in de familie te hebben. Laura, Anke, Peggy en Leonne, ik ben echt ontzettend blij om jullie als vriendinnen te hebben. Laura, er is een speciale plek voor jou in mijn hart. Sinds de middelbare school delen we leuke en verdrietige momenten, belangrijke en minder belangrijke dingen. Van een smaaktest van chocolade eitjes tijdens de Engels les, tot op stap gaan tot in de late uurtjes. Onze vriendschap blijft voor altijd! Anke, wat was ik trots toen ik van dichtbij mocht meemaken hoe jij met verve je proefschrift verdedigde. De treinritjes naar de uni waren een stuk gezelliger samen met jou. De vele koffiemomentjes waren vaak een welkome afleiding op het werk. Daarnaast kan ik me een betere personal coach tijdens een hardloopwedstrijd niet voorstellen. Peggy, bedankt voor alle gezellige zaterdag sportuurtjes, subway dates en je luisterend oor. Leonne, ik vind het altijd fijn om je weer te zien en bij te kletsen over van alles en nog wat en om terug te blikken naar onze vakanties.

Es, Pa en Ken, ik ben zo trots op jullie! Es, lieve zus, the only person who's been were l've been. We hebben samen veel meegemaakt, daardoor we zijn meer dan zussen. Onze vriendschap is onvoorwaardelijk en dat is het mooiste wat er is. Het is zo fijn om iemand te hebben met wie ik alles kan delen, die er altijd voor me is, hoe groot de afstand ook is en met wie ik goed kan shoppen. Ik vind het bewonderenswaardig hoe je voor jullie kleine meid zorgt, mijn lieve dierbare petekindje Elin. Ik ben er trots op dat jij op mijn promotiedag achter me staat, zoals je mijn hele leven al gedaan hebt.

Pa mijn held. Jij was er voor me toen ik je het meest nodig had. Je staat altijd voor me klaar. Een ritje naar het vliegveld of een defecte auto, echt voor alles kan ik je bellen. Als ik het even niet meer weet, ben jij degene die het relativeert. Je enthousiasme en oneindige interesse in mijn werk, hebben me enorm gesteund. Je kwam naar mijn EEG demonstratie op de open dag om te zien wat ik voor een werk doe. lk ga de zaterdag autopoets middagen en het samen eten de komende twee jaar zeker missen.

Ken, als mijn eerste medewerker lay-out heb je een directe bijdrage aan dit proefschrift gehad. Er is niemand waarmee ik mijn voorliefde voor zoveel dingen deel als met jou. Je steunt me in alles wat ik doe en keer op keer verras je me met iets waarvan ik kan leren. Je me laat zien dat er meer is dan wetenschap en je hebt een grenzeloos vertrouwen in alles wat in onderneem en probeer. Alles is gewoon leuker met jou erbij! 


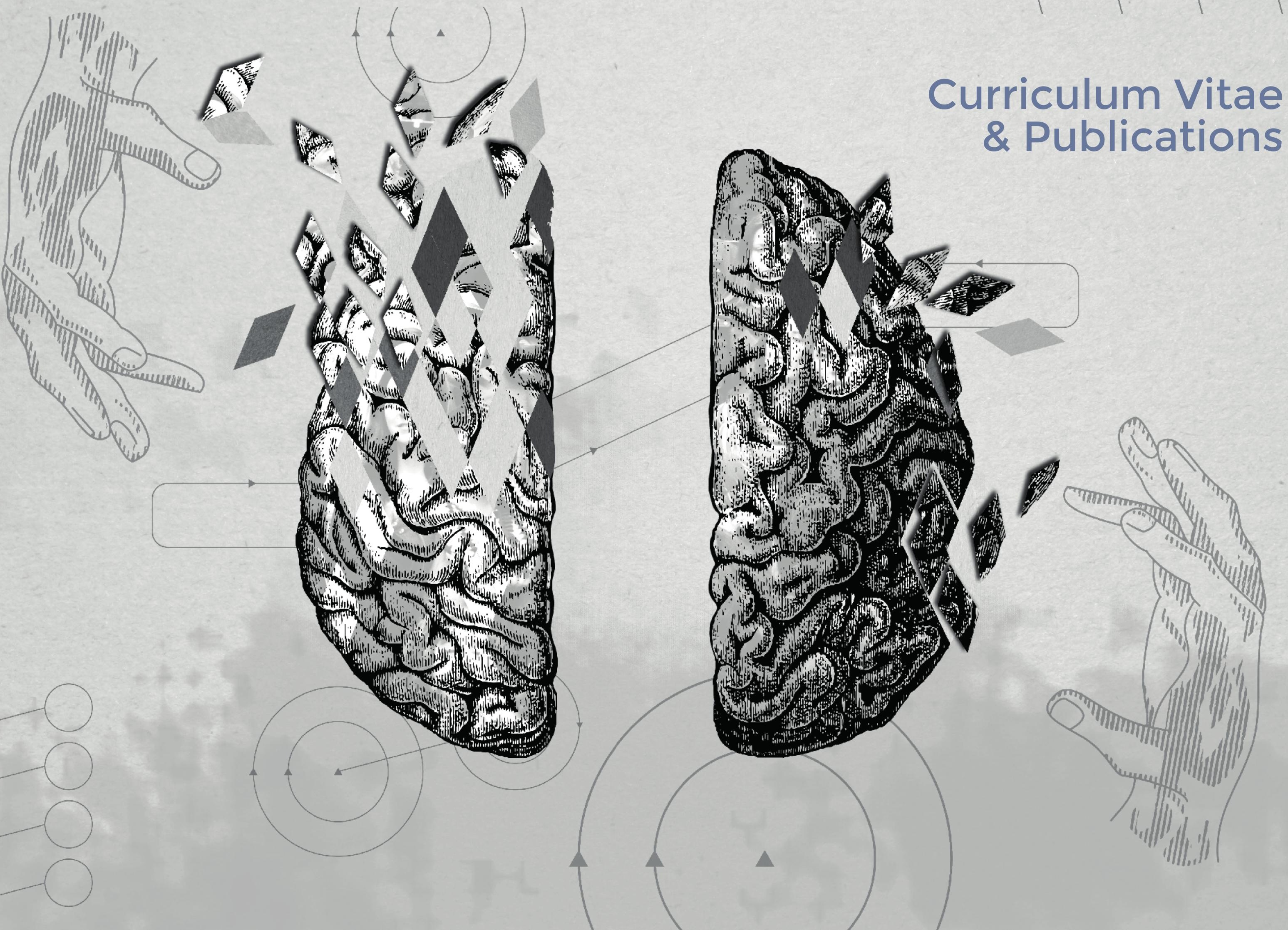




\section{Curriculum Vitae}

Conny Quaedflieg was born on June $19^{\text {th }} 1986$ in Heerlen, the Netherlands. She graduated in 2005 from secondary school (Atheneum, Bernardinus College, Heerlen) and thereafter studied a Bachelor in Biological Psychology at the University of Maastricht. After having obtained her Bachelor of Science degree in Psychology in 2008, she continued with the Research Master Cognitive and Clinical Neuroscience, specialization Neuropsychology. During that time, she also worked as a student-assistant at the Psychopharmacology department, where she was trained as a researcher in combining electroencephalography with psychopharmacological manipulations. During this Master program she performed her research internship in Helsinki (BioMag Laboratory, Helsinki University Central Hospital) under the supervision of Dr. A. Sambeth and Dr. S. Munte. In 2010 she obtained her Master of Science degree (cum laude), after which she worked for two months as a researcher in Helsinki in collaboration with Prof. Dr. E. Kalso. In December 2010 she started a PhD project under the supervision of Prof. Dr. H.L.G.J. Merckelbach and Dr. T. Smeets at the section Forensic Psychology in Maastricht. As of December 2015, she will be working as a postdoctoral fellow in the group of Prof. Dr. L. Schwabe at the University of Hamburg, Germany, were she will investigate the underlying mechanism(s) and interindividual differences in the vulnerability to shift from goal-directed towards habit-based behaviour under stress. For this work she received a Rubicon grant from the Netherlands Organisation for Scientific Research (NWO). 


\section{Publications}

Quaedflieg, C.W.E.M., Smulders, F.T.Y. Meyer, T., Peeters, F., Merckelbach, H \& Smeets, T. (2015). The validity of individual frontal alpha asymmetry EEG neurofeedback. Social Cognitive and Affective Neuroscience.

Quaedflieg, C.W.E.M., van de Ven, V. Meyer, T., Siep, N. \& Smeets, T. (2015). Temporal dynamics of stress-induced alternations of intrinsic amygdala connectivity and neuroendocrine levels. PLoS ONE 10, e0124141. doi:10.1371/journal. pone.0124141

Meyer, T., Smeets, T., Giesbrecht, T. Quaedflieg, C.W.E.M., Smulders, F. T. Y., Meijer, E. H., \& Merckelbach, H. (2015). The role of frontal EEG asymmetry in post-traumatic stress disorder. Biological Psychology, 108, 62-77.

Quaedflieg, C.W.E.M., Meyer, T. Smulders, F.T.Y., \& Smeets, T. (2015). The functional role of individualalpha based frontal asymmetry in stress responding. Biological Psychology, 104, 75-81.
Krix, A.C., Sauerland, M., Raymaekers, L.H.C., Memon, A., Quaedflieg, C.W.E.M., \& Smeets, T. (2015). Eyewitness evidence obtained with the Self-Administered Interview is unaffected by stress. Applied Cognitive Psychology.

Quaedflieg, C.W.E.M., Munte, S., Kalso, E., \& Sambeth, A. (2014). Effects of remifentanil on processing of auditory stimuli: a combined MEG/EEG study. Journal of Psychopharmacology, 28, 39-48.

Meyer, T., Quaedflieg, C.W.E.M., Giesbrecht, T., Meijer, E.H., Abiad, S., \& Smeets, T. (2014). Frontal EEG asymmetry as predictor of physiological responses to aversive memories. Psychophysiology.

Quaedflieg, C.W.E.M., Schwabe, L. Meyer, T., \& Smeets, T. (2013). Time dependent effects of stress prior to encoding on event-related potentials and $24 \mathrm{~h}$ delayed retrieval. Psychoneuroendocrinology, 38, 30573069. 
Quaedflieg, C.W.E.M., Meyer, T., \& Smeets, T. (2013). The imaging Maastricht Acute Stress Test (iMAST): A neuroimaging compatible psychophysiological stressor. Psychophysiology, 50, 758-766.

Münte, S., Quaedflieg, C.W.E.M., Sambeth, A., Wang, M., \& Kalso, E. (2013). Effects of remifentanil on cognitive and psychomotor functioning and mood. British Journal of Anaesthesia, 111, 517-518.

Meyer, T., Smeets, T., Giesbrecht, T., Quaedflieg, C.W.E.M., Girardelli, M. M., Mackay, G.R.N., \& Merckelbach, H. (2013). Individual differences in spatial configuration learning predict the occurrence of intrusive memories. Cognitive Affective \& Behavioral Neuroscience, 13, 186-196.

Meyer, T., Smeets, T., Giesbrecht, T., Quaedflieg, C.W.E.M., \& Merckelbach, H. (2013). Acute stress differentially affects spatial configuration learning in high and low cortisol-responding healthy adults. European Journal of Psychotraumatology, 4.
Quaedflieg, C.W.E.M., Giesbrecht, T., Meijer, E., Merckelbach, H., de Jong, P.J., Thorsteinsson, H., . . Simeon, D. (2013). Early emotional processing deficits in depersonalization: An exploration with event-related potentials in an undergraduate sample. Psychiatry ResearchNeuroimaging, 212, 223-229.

Smeets, T., Cornelisse, S., Quaedflieg, C.W.E.M., Meyer, T., Jelicic, M., \& Merckelbach, H. (2012). Introducing the Maastricht Acute Stress Test (MAST): A quick and non-invasive approach to elicit robust autonomic and glucocorticoid stress responses. Psychoneuroendocrinology, 37, 19982008.

Quaedflieg, C.W.E.M. \& Smeets, T. (2012). Stress Vulnerability Models. In Encyclopedia of Behavioral Medicine (1897-1900): Springer.

Renner, F., Siep, N., Arntz, A., van de Ven, V., Peeters, F.P.M.L., Quaedflieg, C.W.E.M., Huibers, M. J.H. (submitted). Sad mood induction modulates default mode network resting-state functional connectivity in chronic depression. 
